SUSPENDED-SEDIMENT DATA IN THE UPPER RIO GRANDE DE LOIZA BASIN, PUERTO RICO

By Senén Guzmán-Ríos

U.S. GEOLOGICAL SURVEY

Open-File Data Report 88-324

Prepared in cooperation with the

PUERTO RICO DEPARTMENT OF NATURAL RESOURCES, PUERTO RICO ENVIRONMENTAL QUALITY BOARD, PUERTO RICO AQUEDUCT AND SEWER AUTHORITY, UNITED STATES ARMY CORPS OF ENGINEERS, AND THE UNITED STATES DEPARTMENT OF AGRICULTURE, SOIL CONSERVATION SERVICE

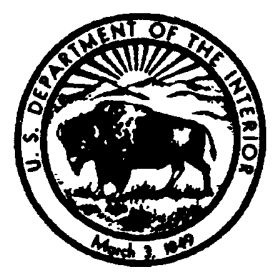

San Juan, Puerto Rico 1989 


\section{UNITED STATES DEPARTMENT OF THE INTERIOR \\ MANUEL LUJAN, Secretary}

\section{U.S. GEOLOGICAL SURVEY}

Dallas L. Peck, Director

For additional information write to:

Chief, Caribbean District, WRD

U.S. Geological Survey

GPO Box 4424

San Juan, Puerto Rico 00936
Copies of this report can be purchased from:

U.S. Geological Survey

Books and Open-File Reports

Federal center, Bldg. 41

Box 25425

Denver, Colorado 80225 
CONTENTS

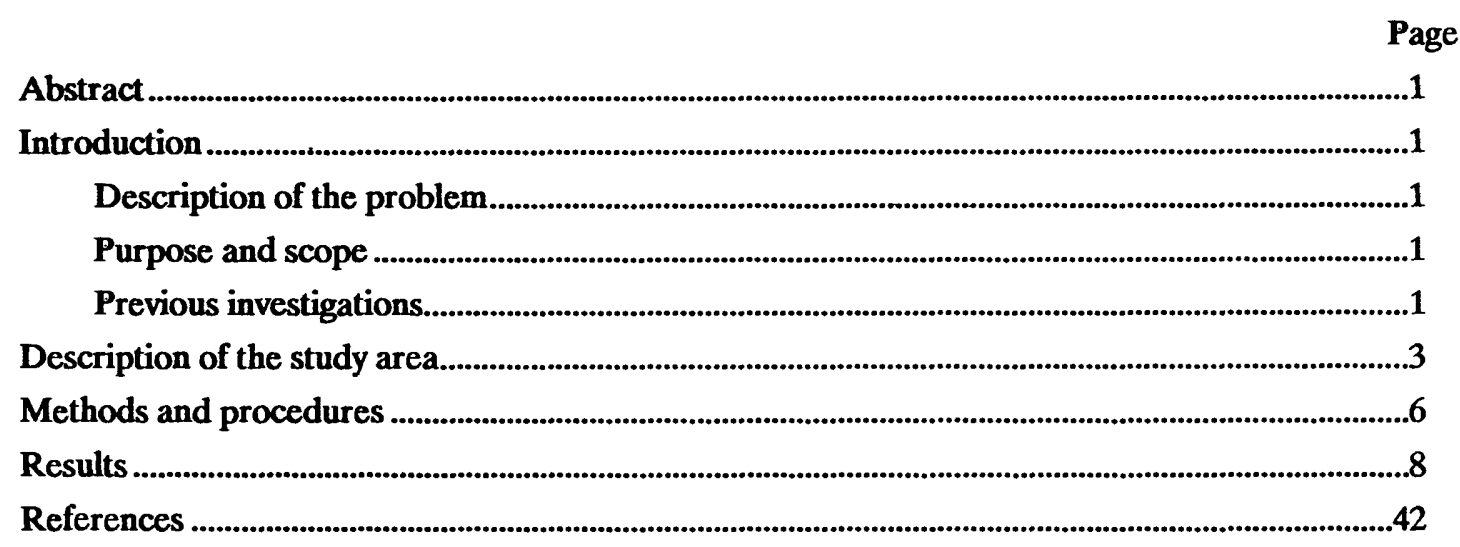

\section{ILLUSTRATIONS}

Figure 1. Map showing the location and study area of the surface water network within the Río Grande de Loiza basin................................................................2

2. Pie diagram showing sources of water supply from the San Juan metropolitan area ......3

3. Map showing surficial geology of the study area .....................................................................5

4-13. Graphs showing relation of suspended-sediment concentration to

4. Río Grande de Loíza at Quebrada Arenas............................................................11

5. Quebrada Blanca at Jagual .......................................................................................13

6. Quebrada Salvatierra near San Lorenzo................................................................15

7. Río Cayaguas at Cerro Gordo .......................................................................................18

8. Río Turabo at Borinquen..............................................................................................22

9. Río Grande de Loíza at Caguas ................................................................................25

10. Quebrada Caimito near Juncos..........................................................................27

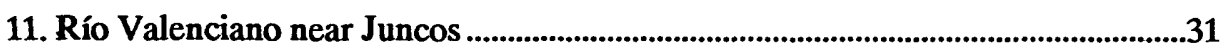

12. Quebrada Mamey near Gurabo .................................................................................33

13. Río Gurabo at Gurabo .........................................................................................35

14. Idealized plot of calibration procedure for pump-sampler data........................................37 


\section{TABLES}

Page

Table 1. Mean monthly rainfall, in inches, at locations in and near the study area

2. Map number, latitude and longitude, drainage areas, and name of stream-gaging stations at the upper Río Grande de Loíza basin

3. Conversion factors $(C)$ for computation of sediment concentrations in milligrams per liter when used with parts per million or the ratio (times $10^{6}$ ) of the weight of sediment to the weight of the water-sediment mixture

4 13. Suspended-sediment data for stations:

4. Río Grande de Loíza at Quebrada Arenas.....................................................................9

5. Quebrada Blanca at Jagual ..............................................................................12

6. Quebrada Salvatierra near San Lorenzo.....................................................................14

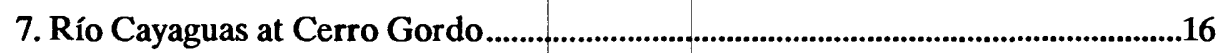

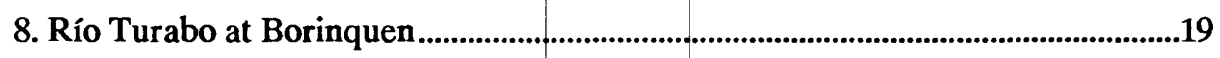

9. Río Grande de Loíza at Caguas ...............................................................................23

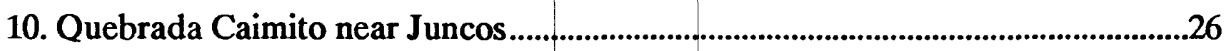

11. Río Valenciano near Juncos ....................................................................................28

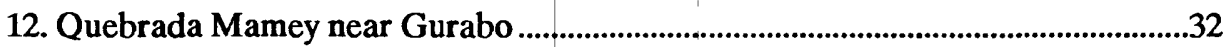

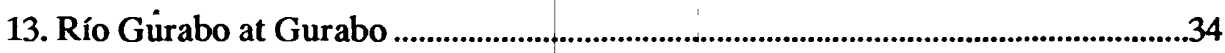

14. Summary of calibrations used for pump-sampler data........................................................36

15. Particle-size distribution data from suspended-sediment samples collected at ten surface-water stations in Río Grande de Loíza basin ...............................38

16. Average water discharge for stations in Río Grande de Loíza basin ................................42

17. Extreme values of water and suspended-sediment data for the upper Río Grande de Loíza basin for the 1984, 1985, and 1986 water years. 


\section{CONVERSION FACTORS}

The following conversion table is included for the convenience of those who prefer to use the SI (International System of Units or metric units) rather than the inch-pound system of units. Concentrations of chemical parameters are given in milligrams per liter $(\mathrm{mg} / \mathrm{L})$, which are for the values presented numerically equal to parts per million (ppm). Specific conductance values are given in microsiemens per centimeter at 25 degrees Celsius (S/cm at $25 \mathrm{C}$ ).

Multiply inch-pound unit

inch (in.)

foot (ft)

mile (mi)

acre

acre

square mile $\left(\mathrm{mi}^{2}\right)$

pound per cubic foot

$\left(\mathrm{lb} / \mathrm{ft}^{3}\right)$

short ton per day

(short ton/d)

ton per square mile

per year $\left[\left(\right.\right.$ ton $\left.\left./ \mathrm{mi}^{2}\right) / \mathrm{yr}\right]$

gallons (gal)

acre-foot (acre-ft)

cubic foot $\left(\mathrm{ft}^{3}\right)$

cubic yard $\left(\mathrm{yd}^{3}\right)$

cubic mile $\left(\mathrm{mi}^{3}\right)$

million gallons (Mgal)

gallons per minute

(gal/min)

cubic foot per second

$\left(\mathrm{ft}^{3} / \mathrm{s}\right)$

million gallons per day

(Mgal/d)

degrees Farenheit $\left({ }^{\circ} \mathrm{F}\right)$
By

Length

25.4

0.3048

1.609

Area

4,047

0.4047

2.590

Mass Per Unit Time

16.02

0.9072

0.3503

Volume

3.785

1,233

0.02832

0.7646

4.168

3,785

Volume Per Unit Time

0.06308

0.02832

0.04381

Temperature

${ }^{\circ} \mathrm{C}=5 / 9 \times\left({ }^{\circ} \mathrm{F}-32\right)$
Te obtain metric units

millimeter (mm)

meter (m)

kilometer (km)

square meter $\left(\mathrm{m}^{2}\right)$

hectare (ha)

square kilometer $\left(\mathrm{km}^{2}\right)$

kilogram per cubic meter

$\left(\mathrm{kg} / \mathrm{m}^{3}\right)$

metric ton per day ( $t / d)$

tonnes per day $(\mathrm{td} / \mathrm{d})$

metric ton per square

kilometer per year $\left(\mathrm{t} / \mathrm{km}^{2}\right) / \mathrm{yr}$

or $(\mathrm{mg} / \mathrm{km} 2) / \mathrm{yr}$

liters (L)

cubic meter $\left(\mathrm{m}^{3}\right)$

cubic meter $\left(\mathrm{m}^{3}\right)$

cubic meter $\left(\mathrm{m}^{3}\right)$

cubic kilometer $\left(\mathrm{km}^{3}\right)$

cubic meter $\left(\mathrm{m}^{3}\right)$

liters per second $(\mathrm{L} / \mathrm{s})$

cubic meter per second

$\left(\mathrm{m}^{3} / \mathrm{s}\right)$

cubic meter per second

$\left(\mathrm{m}^{3} / \mathrm{s}\right)$

degrees Celsius $\left({ }^{\circ} \mathrm{C}\right)$ 


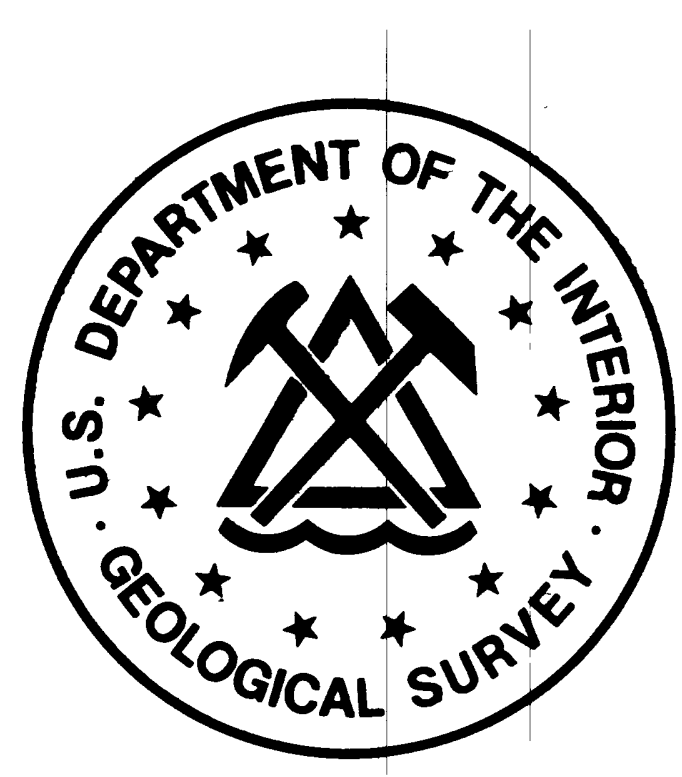




\title{
SUSPENDED-SEDIMENT DATA IN THE UPPER RIO GRANDE DE LOIZA BASIN, PUERTO RICO
}

\author{
By Senén Guzmán-Ríos
}

\begin{abstract}
This report covers the period from 1983 to 1986 and presents data on suspended-sediment transport from ten small- to medium-sized drainage areas in eastcentral Puerto Rico. The drainage areas range from 0.82 square miles to 89.8 square miles. Results are based on three years of data from a network of five daily-record stations and five partial-record stations.

A total of 2,114 sediment samples were collected and analyzed during the investigation. Instantaneous concentrations of suspended-sediment varied from 0 to 56,100 milligrams per liter. Instantaneous suspendedsediment loads ranged from 0 tons per day to 817,000 tons per day. A total of 152 suspended-sediment samples were analyzed for particle size distribution. Suspended-sediment in the streams is composed mostly of silt and clay. Sand content ranged from 6 to 80 percent during high flows.
\end{abstract}

\section{INTRODUCTION}

In 1983, the U.S. Geological Survey, Water Resources Division, in cooperation with the Puerto Rico Department of Natural Resources (PRDNR), Puerto Rico Environmental Quality Board (PREQB), Puerto Rico Aqueduct and Sewer Authority (PRASA), U.S. Army Corps of Engineers (USCOE), and the U.S. Department of Agriculture/ Soil Conservation Service (USSCS), started an investigation in an effort to answer the following questions concerning sediment transport in the Río Grande de Loíza basin.

1. Where and when is the sediment deposited?

2. Is the amount of sediment deposited during low stages the same as the amount resuspended during high flows?

3. How is suspended-sediment movement related to changes in flow conditions?

\section{Description of the Problem}

Fluvial sediment, a widely recognized pollutant of surface water, is reducing the efficiency and useful life of almost all reservoirs in Puerto Rico. Sediment tranported from the upper basin of the Río Grande de Loíza is eventually deposited in the bottom of Lago
Loíza, a water supply reservoir (fig. 1). The reservoir is being significantly affected by the sediment deposition. This reservoir supplies about 50 percent of the water for the San Juan Metropolitan area (fig. 2).

Because of the importance of the basin for water supply, plans are being developed for dams on three tributaries: Río Grande de Loíza at Quebrada Arenas, Río Cayaguas at Cerro Gordo, and Río Valenciano near Juncos (fig. 1). The useful life of Lago Loiza and the design and construction of the three reservoirs will be greatly influenced by sediment transport and deposition.

Collection and analysis of suspended-sediment transport and deposition data in conjunction with landuse data can be used to implement mitigation practices in the basin in an effort to reduce erosion and sedimentation rates.

\section{Purpose and Scope}

The purpose of this report is to summarize the suspended-sediment data collected during the data collection phase of the study. Data from ten suspended-sediment stations are presented for the period of 1983 to 1986 . The study area of 208 square miles extends from the headwaters of Río Grande de Loíza to the Lago Loíza. Data were collected from ten streamflow and suspended-sediment stations established along the main stream and selected tributaries.

A final interpretative report entitled "Sediment Transport and Yields in the upper Río Grande de Loíza Basin, Puerto Rico," will be published at the end of the investigation. In the final report, sediment transport and its delivery to Lago Loíza will be addressed.

\section{Previous Investigations}

No previous studies have been conducted to assess sediment transport in the upper part of the Río Grande de Loíza basin. Few sediment samples were collected at fixed stations along the basin prior to this study. However, some studies have been conducted on sedimentation in the Lago Loíza area (QuiñonesMarquez, 1980). Results indicate that sedimentation and channel-bed movement in the Río Grande de Loíza are constant threats to the useful life of Lago 


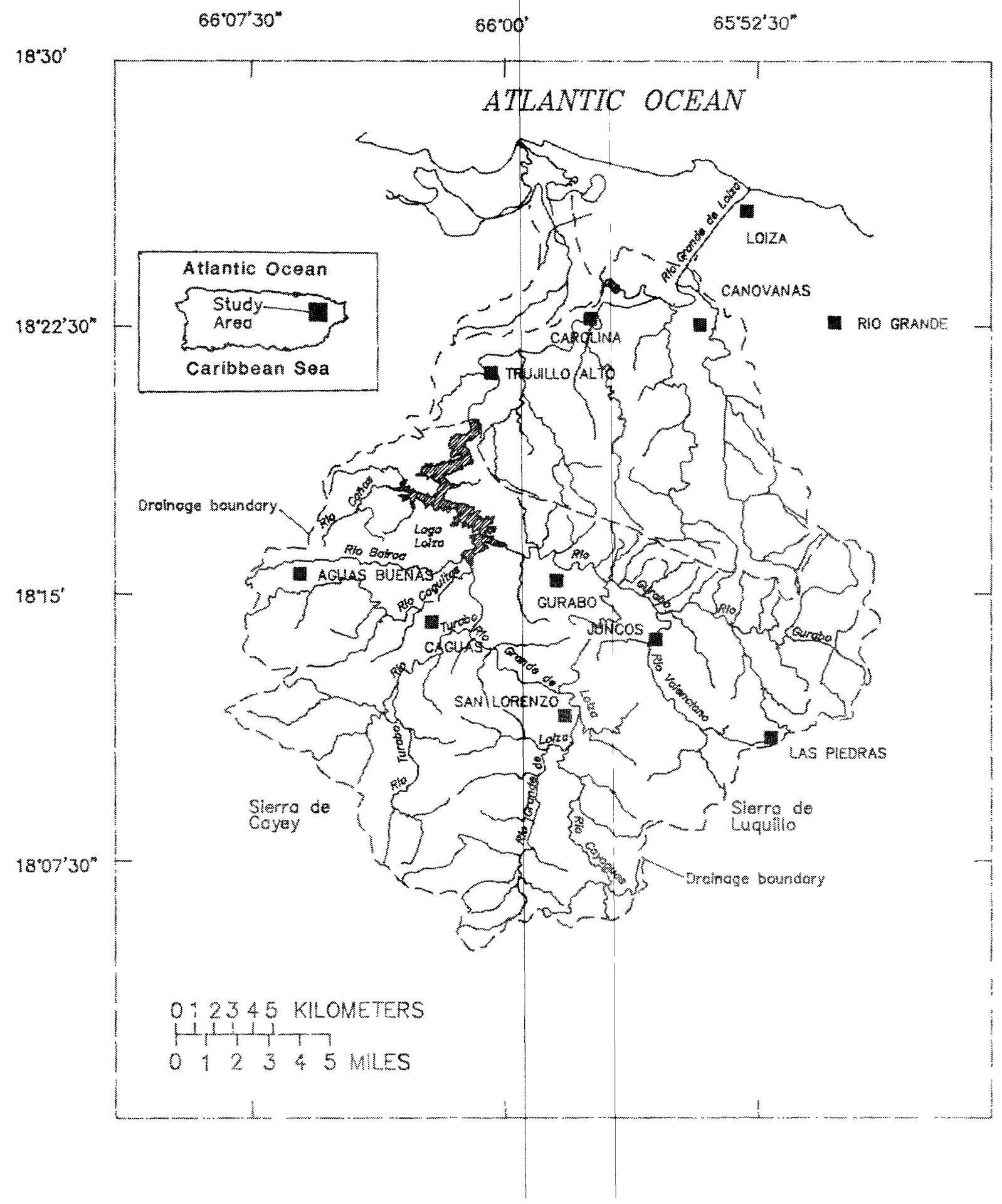




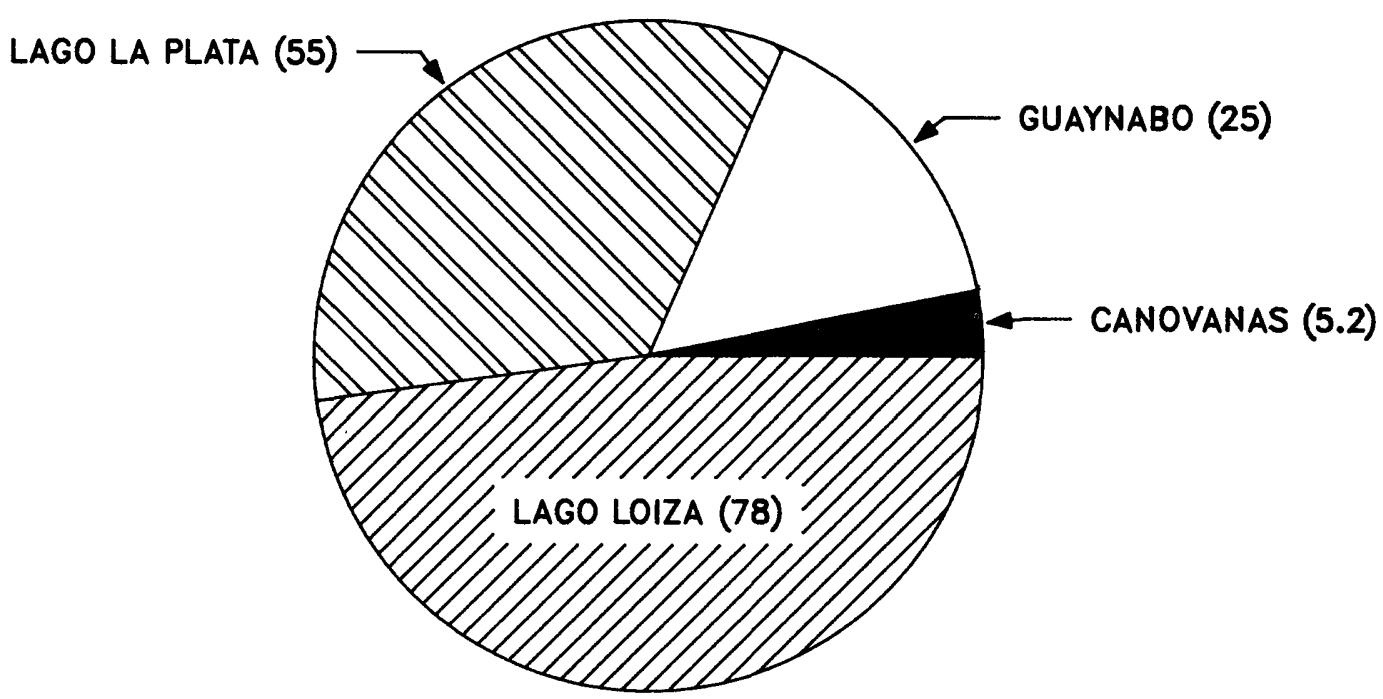

Figure 2.--Sources of water supply for the San Juan metropolitan area. Numbers in parenthesis are in million gallons per day.

Loíza. Some bathymetric surveys of Lago Loíza have been made by the U.S. Soil Conservation Service (Iivari, 1981). A recent sedimentation survey of Lago Loíza has shown a capacity loss rate of 1.8 percent per year (Quiñones-Marquez and others, 1987). Preliminary results of that study indicate that silts and clays transported from the upper basin account for most of the sediments deposited in the reservoir. However, recent coring of Lago Loíza by the U.S. Geological Survey (1989) has disclosed sand deposits throughout the upper 3 feet of bottom sediments.

\section{DESCRIPTION OF THE STUDY AREA}

The Río Grande de Loíza basin is the largest drainage basin in Puerto Rico. It is in east-central Puerto Rico (fig. 1) and is among the most developed watersheds on the island. Río Grande de Loíza, which is the largest stream in drainage area and third largest in water discharge in Puerto Rico, was dammed in 1953 to build a water-supply reservoir. Lago Loíza (also known locally as Lago Carraizo), is the only reservoir in the basin and one of the biggest water-supply sources on the island. It had a storage capacity of about 10,000 acre-ft in 1985 and supplied about 110 million gallons per day (Mgal/d) of raw water to the Sergio Cuevas public water supply filtration plant. Outflow from this plant to the San Juan Metropolitan area is reported to average $78 \mathrm{Mgal} / \mathrm{d}$ (fig. 2).

The basin is characterized by periods of intense rainfall during the relatively wet seasons from August to November and April to June. Relatively dry periods occur from December to March and from June to July. Mean annual rainfall ranges from 63 to 100 inches per year. Higher rainfall occurs in the headwater areas than in the alluvial valleys due to orographic effects. Data obtained from National Oceanographic and Atmospheric Administration (NOAA) for the period 1983 to 1986 are summarized in table 1.

The basin is also characterized by mountainous terrain covered by dense vegetation. The boundary of the upper basin is marked by the Sierra de Cayey in the southeast, while the Sierra de Luquillo, in the northeast, defines the middle-lower basin (fig. 3). Geologically, the basin is dominated by plutonic rocks, largely granodiorite and quartz diorite (Briggs and Akers, 1965). Locally those formations are deeply weathered. Lava, lava breccia, and tuff and tuffaceous breccia largely deposited in a marine environment occur in rest of the basin. Extensive alluvial valleys are found near Lago Loíza.

The total drainage area covered by the study is 208 square miles. The network of sampling stations and the area draining to each station are summarized in table 2.

The basin is largely agricultural and industrial. Sugarcane, until recently the principal crop, has been replaced by pastureland and truck-farm crops. Industrial activities are centered near Caguas, Las Piedras, and Juncos (fig. 1). 
Table 1. Mean monthly rainfall, in inches, at locations in and near the Rio Grande de Loiza basin

[M. Missing data; - Mean was not computed due to insufficient amount of data; *, All stations, except La Muda-Caguas and Trujillo Alto $2 \mathrm{SSW}$, are located in the Eastern Interior according National 0ceanographic and atmospheric Administration (NOAA) map. These two stations are part of the Northern Slopes.]

\begin{tabular}{|c|c|c|c|c|c|c|c|c|c|c|c|c|c|}
\hline Station Name* & $\begin{array}{l}\text { Water } \\
\text { Year }\end{array}$ & $0 \mathrm{ct}$ & Nov & Dec & Jan & Feb & Mar & Apr & May & June & July & Aug & Sept \\
\hline \multirow[t]{2}{*}{ Caguas $1 \mathrm{~W}$} & $\begin{array}{l}1983 \\
1984 \\
1985 \\
1986 \\
1987\end{array}$ & $\begin{array}{r}1.75 \\
6.38 \\
6.39 \\
21.00 \\
4\end{array}$ & $\begin{array}{r}7.63 \\
5.39 \\
16.97 \\
9.53 \\
15.16\end{array}$ & $\begin{array}{l}7.34 \\
3.67 \\
3.66 \\
1.78 \\
\mathrm{H}\end{array}$ & $\begin{array}{r}1.80 \\
1.44 \\
.52 \\
1.09 \\
2.87\end{array}$ & $\begin{array}{l}2.34 \\
6.04 \\
1.90 \\
3.15 \\
3.61\end{array}$ & $\begin{array}{r}3.01 \\
.35 \\
6.42 \\
1.73 \\
2.80\end{array}$ & $\begin{array}{r}5.82 \\
.85 \\
3.66 \\
10.59 \\
6.35\end{array}$ & $\begin{array}{r}5.13 \\
7.70 \\
11.08 \\
16.67 \\
M\end{array}$ & $\begin{array}{l}1.61 \\
3.78 \\
2.82 \\
2.55 \\
M\end{array}$ & $\begin{array}{r}7.77 \\
5.11 \\
2.09 \\
.92 \\
.22\end{array}$ & $\begin{array}{l}7.71 \\
4.54 \\
5.99 \\
9.56 \\
2.32\end{array}$ & $\begin{array}{c}10 \\
10.98 \\
8.39 \\
2.51 \\
5.69\end{array}$ \\
\hline & Mean & - & 10.94 & - & 1.54 & 3.41 & 2.86 & 5.45 & - & - & 3.22 & 6.02 & - \\
\hline \multirow[t]{2}{*}{ Cayey $1 \mathrm{E}$} & $\begin{array}{l}1983 \\
1984 \\
1985 \\
1986 \\
1987\end{array}$ & $\begin{array}{r}4.29 \\
1.66 \\
5.56 \\
24.43 \\
4.22\end{array}$ & $\begin{array}{r}7.78 \\
4.12 \\
19.76 \\
7.28 \\
10.79\end{array}$ & $\begin{array}{l}3.91 \\
2.43 \\
3.74 \\
1.86 \\
2.95\end{array}$ & $\begin{array}{l}2.38 \\
3.24 \\
1.13 \\
2.38 \\
3.57\end{array}$ & $\begin{array}{l}.63 \\
3.70 \\
3.21 \\
1.33 \\
3.63\end{array}$ & $\begin{array}{r}3.74 \\
.72 \\
4.45 \\
2.22 \\
1.50\end{array}$ & $\begin{array}{l}5.76 \\
2.49 \\
7.51 \\
8.34 \\
4.61\end{array}$ & $\begin{array}{r}4.87 \\
2.55 \\
15.64 \\
15.38 \\
5.72\end{array}$ & $\begin{array}{r}6.59 \\
2.71 \\
.51 \\
2.49 \\
6.33\end{array}$ & $\begin{array}{l}6.11 \\
5.30 \\
4.10 \\
1.89 \\
5.45\end{array}$ & $\begin{array}{l}6.68 \\
1.83 \\
4.14 \\
7.09 \\
3.12\end{array}$ & $\begin{array}{l}2.83 \\
9.69 \\
7.35 \\
3.82 \\
3.55\end{array}$ \\
\hline & Mean & 8.03 & 9.95 & 2.98 & 2.54 & 2.50 & 2.53 & 5.74 & 8.83 & 3.73 & 4.57 & 4.57 & 5.45 \\
\hline \multirow[t]{2}{*}{ Cidra $1 \mathrm{E}$} & $\begin{array}{l}1983 \\
1984 \\
1985 \\
1986 \\
1987\end{array}$ & $\begin{array}{r}2.67 \\
2.95 \\
5.12 \\
22.40 \\
1.77\end{array}$ & $\begin{array}{r}5.04 \\
4.84 \\
18.46 \\
8.61 \\
10.35\end{array}$ & $\begin{array}{l}7.66 \\
3.68 \\
5.85 \\
1.46 \\
3.59\end{array}$ & $\begin{array}{l}2.90 \\
3.31 \\
1.47 \\
2.36 \\
3.64\end{array}$ & $\begin{array}{l}.81 \\
4.91 \\
3.07 \\
1.63 \\
5.63\end{array}$ & $\begin{array}{r}3.90 \\
.81 \\
6.21 \\
2.61 \\
.81\end{array}$ & $\begin{array}{l}5.29 \\
3.58 \\
5.24 \\
5.07 \\
7.33\end{array}$ & $\begin{array}{r}8.53 \\
5.97 \\
12.30 \\
18.57 \\
6.23\end{array}$ & $\begin{array}{r}1.83 \\
8.37 \\
.12 \\
2.23 \\
10.16\end{array}$ & $\begin{array}{c}6.68 \\
8.96 \\
3.42 \\
M \\
4.85\end{array}$ & $\begin{array}{l}6.92 \\
4.34 \\
3.48 \\
6.35 \\
4.00\end{array}$ & $\begin{array}{l}5.26 \\
H^{7.19} \\
3.08 \\
4.00\end{array}$ \\
\hline & Mean & 6.98 & 9.46 & 4.45 & 2.74 & 3.21 & 2.87 & 5.30 & 10.32 & 4.54 & $\cdot$ & 5.02 & - \\
\hline \multirow[t]{2}{*}{ Guavate Camp } & $\begin{array}{l}1983 \\
1984 \\
1985 \\
1986 \\
1987\end{array}$ & $\begin{array}{r}6.70 \\
4.39 \\
11.04 \\
14 \\
6.58\end{array}$ & $\begin{array}{r}8.80 \\
4.96 \\
34.45 \\
14.89\end{array}$ & $\begin{array}{l}7.78 \\
3.97 \\
M \\
M\end{array}$ & $\begin{array}{c}5.55 \\
8.93 \\
M \\
M\end{array}$ & $\begin{array}{l}2.20 \\
B .84 \\
M \\
M\end{array}$ & $\begin{array}{c}2.68 \\
2.58 \\
M \\
M \\
8.37\end{array}$ & $\begin{array}{l}M \\
M \\
M \\
M \\
M\end{array}$ & $\begin{array}{r}10.26 \\
3.85 \\
10.00 \\
17.85 \\
8.75\end{array}$ & $\begin{array}{l}M \\
M \\
.13 \\
7.36 \\
4.74\end{array}$ & $\begin{array}{c}11.13 \\
M \\
8.22 \\
3.83 \\
2.29\end{array}$ & $\begin{array}{r}11.99 \\
4.68 \\
5.07 \\
8.32 \\
2.97\end{array}$ & $\begin{array}{r}5.10 \\
15.42 \\
10.23 \\
2.86 \\
3.34\end{array}$ \\
\hline & Mean & - & - & - & - & - & - & - & 10.14 & - & - & 6.61 & 7.39 \\
\hline \multirow[t]{2}{*}{ Gurabo Substation } & $\begin{array}{l}1983 \\
1984 \\
1985 \\
1986 \\
1987\end{array}$ & $\begin{array}{r}2.18 \\
7.39 \\
6.05 \\
18.74 \\
6.11\end{array}$ & $\begin{array}{l}6.35 \\
5.23 \\
9.79 \\
7.07 \\
8.48\end{array}$ & $\begin{array}{l}6.38 \\
5.66 \\
3.21 \\
2.39 \\
3.76\end{array}$ & $\begin{array}{r}1.13 \\
3.60 \\
.87 \\
1.88 \\
2.15\end{array}$ & $\begin{array}{l}1.34 \\
6.04 \\
2.07 \\
2.16 \\
3.16\end{array}$ & $\begin{array}{r}3.00 \\
.90 \\
4.78 \\
2.95 \\
1.96\end{array}$ & $\begin{array}{l}9.10 \\
2.03 \\
3.55 \\
6.24 \\
3.75\end{array}$ & $\begin{array}{r}4.37 \\
5.15 \\
12.49 \\
12.52 \\
9.25\end{array}$ & $\begin{array}{r}2.97 \\
5.27 \\
2.48 \\
2.56 \\
13.89\end{array}$ & $\begin{array}{l}9.52 \\
5.34 \\
4.60 \\
2.09 \\
2.30\end{array}$ & $\begin{array}{l}9.53 \\
2.86 \\
8.35 \\
8.30 \\
4.17\end{array}$ & $\begin{array}{r}2.62 \\
7.78 \\
10.05 \\
2.71 \\
6.69\end{array}$ \\
\hline & Mean & 8.09 & 7.38 & 4.28 & 1.93 & 2.95 & 2.72 & 4.93 & 8.76 & 5.43 & 4.77 & 6.64 & 5.97 \\
\hline \multirow[t]{2}{*}{ Juncos 1 NNE } & $\begin{array}{l}1983 \\
1984 \\
1985 \\
1986 \\
1987\end{array}$ & $\begin{array}{c}5.68 \\
M \\
6.06 \\
20.68 \\
4.43\end{array}$ & $\begin{array}{c}3.93 \\
13.00 \\
6.38 \\
7.78\end{array}$ & $\begin{array}{l}1.86 \\
\begin{array}{l}M .88 \\
1.96 \\
2.18\end{array}\end{array}$ & $\begin{array}{l}3.13 \\
M \\
1.28 \\
2.19 \\
3.39\end{array}$ & $\begin{array}{l}.53 \\
\dot{H}^{.82} \\
.39 \\
3.23\end{array}$ & $\begin{array}{l}2.74 \\
M \\
1.60 \\
3.16 \\
3.05\end{array}$ & $\begin{array}{l}8.89 \\
M \\
4.36 \\
5.52 \\
6.27\end{array}$ & $\begin{array}{r}4.18 \\
17.31 \\
13.47 \\
7.94\end{array}$ & $\begin{array}{r}4.13 \\
1.50 \\
.35 \\
4.23 \\
12.62\end{array}$ & $\begin{array}{l}8.56 \\
4.87 \\
6.69 \\
3.71 \\
2.76\end{array}$ & $\begin{array}{r}14.87 \\
6.50 \\
4.67 \\
7.13 \\
1.92\end{array}$ & $\begin{array}{r}1.15 \\
7.17 \\
11.34 \\
1.91 \\
5.14\end{array}$ \\
\hline & Mean & - & - & - & - & - & - & - & - & 4.57 & 5.32 & 7.02 & 5.34 \\
\hline \multirow[t]{2}{*}{ San Lorenzo $3 \mathrm{~S}$} & $\begin{array}{l}1983 \\
1984 \\
1985 \\
1986 \\
1987\end{array}$ & $\begin{array}{r}8.22 \\
4.98 \\
10.80 \\
26.39 \\
9.11\end{array}$ & $\begin{array}{r}10.85 \\
11.78 \\
18.35 \\
6.16 \\
8.72\end{array}$ & $\begin{array}{l}6.77 \\
6.35 \\
7.58 \\
7.36 \\
4.88\end{array}$ & $\begin{array}{l}4.69 \\
4.83 \\
2.64 \\
4.38 \\
6.34\end{array}$ & $\begin{array}{l}1.79 \\
8.96 \\
4.41 \\
2.18 \\
4.92\end{array}$ & $\begin{array}{l}6.92 \\
2.21 \\
7.34 \\
6.05 \\
3.83\end{array}$ & $\begin{array}{l}7.60 \\
3.17 \\
9.94 \\
7.89 \\
5.45\end{array}$ & $\begin{array}{r}7.39 \\
9.64 \\
19.49 \\
17.72 \\
8.98\end{array}$ & $\begin{array}{r}11.08 \\
13.12 \\
1.31 \\
8.55 \\
14.86\end{array}$ & $\begin{array}{r}19.10 \\
8.89 \\
13.73 \\
6.66 \\
6.67\end{array}$ & $\begin{array}{r}13.07 \\
6.76 \\
6.95 \\
11.02 \\
5.01\end{array}$ & $\begin{array}{r}9.25 \\
14.17 \\
15.36 \\
6.10 \\
5.17\end{array}$ \\
\hline & Mean & 11.90 & 11.17 & 6.59 & 4.58 & 4.45 & 5.27 & 6.81 & 12.64 & 9.78 & 11.01 & 8.56 & 10.01 \\
\hline \multirow[t]{2}{*}{ San Lorenzo Farm 2 NW } & $\begin{array}{l}1983 \\
1984 \\
1985 \\
1986 \\
1987\end{array}$ & $\begin{array}{r}6.58 \\
3.50 \\
4.29 \\
21.21 \\
4.47\end{array}$ & $\begin{array}{r}5.98 \\
7.63 \\
14.24 \\
6.68 \\
8.26\end{array}$ & $\begin{array}{l}4.26 \\
3.68 \\
4.06 \\
2.49 \\
3.11\end{array}$ & $\begin{array}{l}2.00 \\
2.85 \\
1.58 \\
2.71 \\
4.15\end{array}$ & $\begin{array}{l}.49 \\
.88 \\
2.47 \\
.09 \\
.34\end{array}$ & $\begin{array}{r}4.59 \\
.55 \\
7.74 \\
3.67 \\
2.88\end{array}$ & $\begin{array}{l}5.04 \\
1.13 \\
5.26 \\
9.95 \\
2.27\end{array}$ & $\begin{array}{r}5.86 \\
5.91 \\
15.94 \\
10.98 \\
6.20\end{array}$ & $\begin{array}{r}5.14 \\
9.56 \\
.42 \\
5.40 \\
12.64\end{array}$ & $\begin{array}{l}9.30 \\
5.20 \\
9.39 \\
2.82 \\
3.03\end{array}$ & $\begin{array}{r}13.16 \\
5.61 \\
4.68 \\
7.93 \\
3.77\end{array}$ & $\begin{array}{r}5.20 \\
8.84 \\
12.28 \\
2.71 \\
4.77\end{array}$ \\
\hline & Mean & 8.01 & 8.56 & 3.52 & 2.66 & 2.65 & 3.89 & 4.73 & 8.98 & 6.63 & 5.95 & 7.03 & 6.76 \\
\hline \multirow[t]{2}{*}{ La Muda - Caguas } & $\begin{array}{l}1983 \\
1984 \\
1985 \\
1986 \\
1987\end{array}$ & $\begin{array}{r}4.37 \\
11.36 \\
8.47 \\
19.13 \\
7.71\end{array}$ & $\begin{array}{r}8.28 \\
8.16 \\
10.63 \\
10.02 \\
9.33\end{array}$ & $\begin{array}{l}8.11 \\
4.33 \\
6.56 \\
2.54 \\
3.54\end{array}$ & $\begin{array}{l}.47 \\
1.49 \\
3.24 \\
2.77 \\
M\end{array}$ & $\begin{array}{r}1.55 \\
.27 \\
3.90 \\
.88 \\
.02\end{array}$ & $\begin{array}{r}1.93 \\
.46 \\
6.91 \\
2.52 \\
1.91\end{array}$ & $\begin{array}{r}5.86 \\
.00 \\
4.67 \\
11.74 \\
9.17\end{array}$ & $\begin{array}{c}M \\
6.87 \\
7.69 \\
12.16 \\
11.64\end{array}$ & $\begin{array}{l}1.04 \\
5.74 \\
2.76 \\
.33 \\
8.68\end{array}$ & $\begin{array}{r}11.36 \\
12.48 \\
2.88 \\
6.89 \\
6.11\end{array}$ & $\begin{array}{r}8.16 \\
4.35 \\
7.00 \\
11.01 \\
7.23\end{array}$ & $\begin{array}{l}4.33 \\
8.20 \\
7.05 \\
1.32 \\
4.44\end{array}$ \\
\hline & Mean & 10.21 & 9.28 & 5.02 & - & 3.52 & 2.75 & 6.29 & - & 3.71 & 7.94 & 7.55 & 5.07 \\
\hline \multirow[t]{2}{*}{ Irujillo Alto $2 \mathrm{SSW}$} & $\begin{array}{l}1983 \\
1984 \\
1985 \\
1986 \\
1987\end{array}$ & $\begin{array}{r}1.56 \\
8.36 \\
7.48 \\
13.83 \\
4.88\end{array}$ & $\begin{array}{l}5.76 \\
5.94 \\
8.05 \\
9.36 \\
8.73\end{array}$ & $\begin{array}{l}5.95 \\
5.48 \\
7.07 \\
1.78 \\
2.86\end{array}$ & $\begin{array}{l}.97 \\
2.91 \\
3.24 \\
2.84 \\
2.69\end{array}$ & $\begin{array}{l}.66 \\
.77 \\
3.70 \\
1.61 \\
4.47\end{array}$ & $\begin{array}{l}1.90 \\
2.16 \\
5.51 \\
2.28 \\
4.02\end{array}$ & $\begin{array}{r}5.76 \\
.61 \\
2.80 \\
5.28 \\
13.29\end{array}$ & $\begin{array}{r}8.04 \\
8.27 \\
7.57 \\
11.48 \\
M\end{array}$ & $\begin{array}{r}1.82 \\
3.21 \\
11.11 \\
1.61 \\
10.76\end{array}$ & $\begin{array}{l}8.33 \\
5.95 \\
4.72 \\
4.30 \\
2.88\end{array}$ & $\begin{array}{r}10.65 \\
4.11 \\
7.48 \\
7.51 \\
6.29\end{array}$ & $\begin{array}{r}2.19 \\
13.90 \\
10.10 \\
1.75 \\
5.72\end{array}$ \\
\hline & Mean & 7.22 & 7.57 & 4.63 & 2.53 & 3.44 & 3.17 & 5.55 & - & 5.70 & 5.24 & 7.21 & 6.73 \\
\hline
\end{tabular}




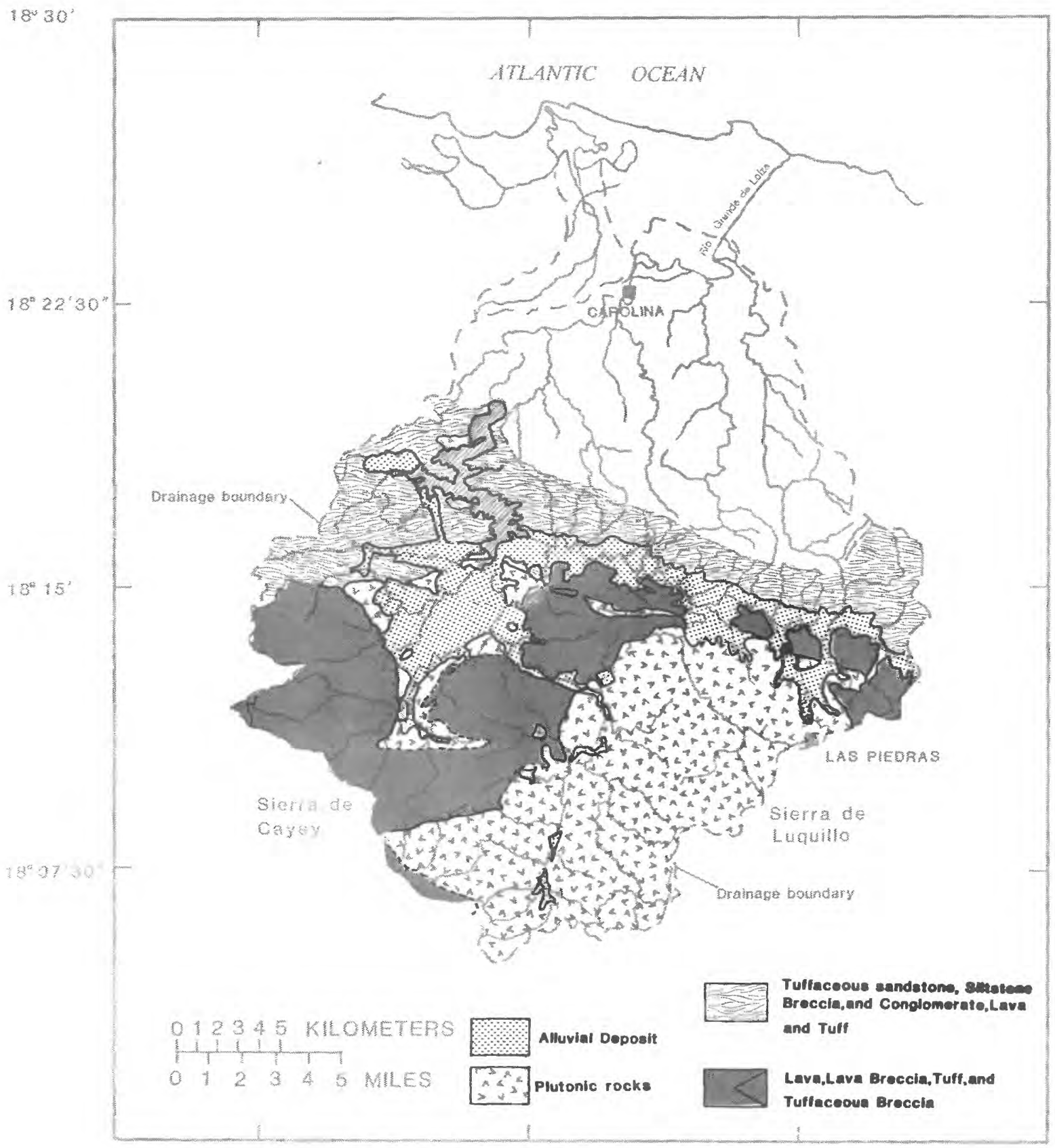

Figure 3.--Surficial geology of the study area 


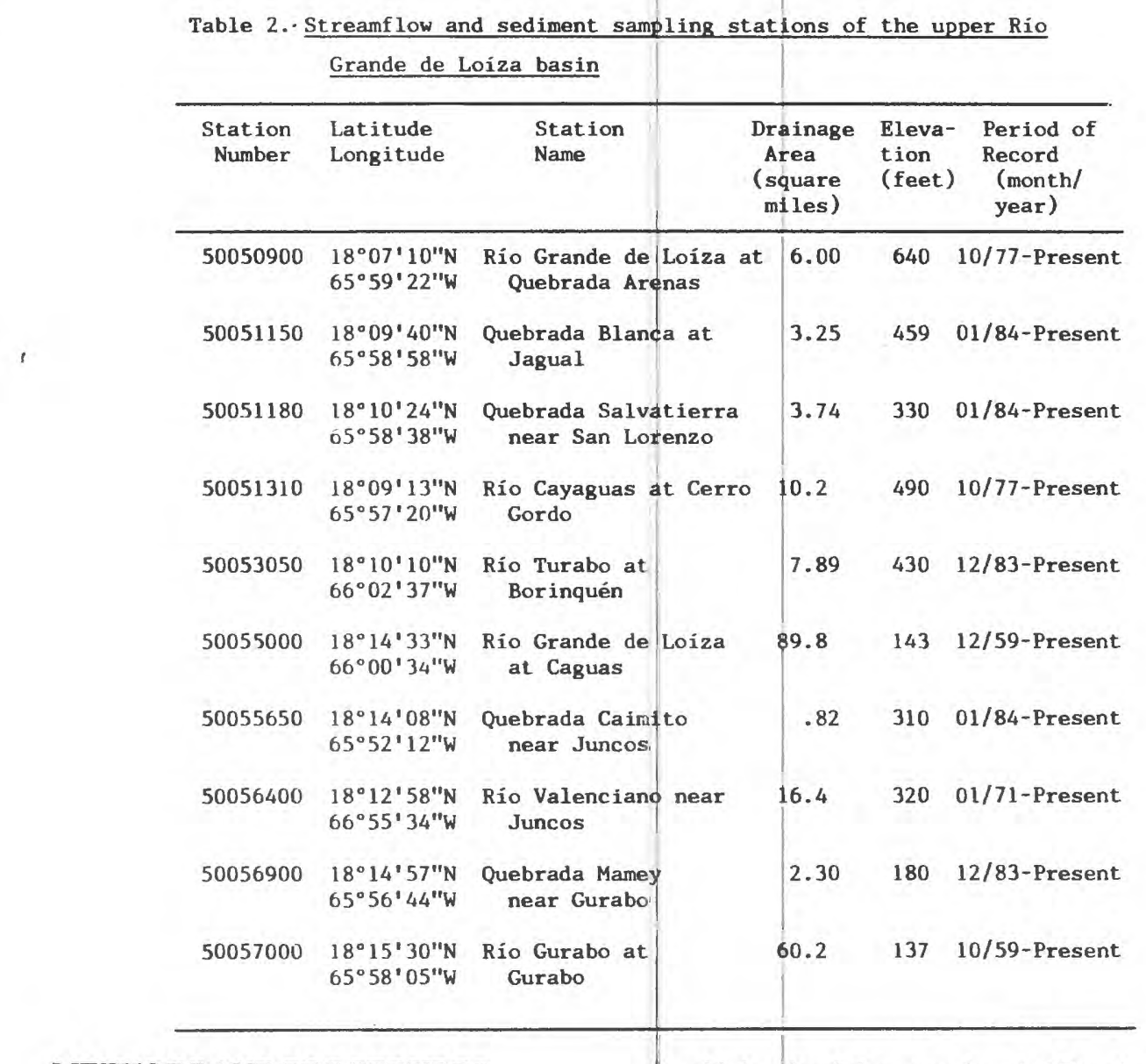

\section{METHODS AND PROCEDURES}

Many direct and some indirect measurements of streamflow and stage were made over a range of conditions to define the relationship between the elevation of the water surface (stage) and water discharge at each gaging station. This relationship, called a rating curve, is used to estimate instantaneous water discharge when only stage is known (Rantz and others, 1982). Stage values were obtained from automatic digital recorders (ADR) installed at each gaging station using a 15-minute recording interval. These 15-minute data were then used to compute mean daily streamflows.

Suspended-sediment samples were collected one or two times per week at each station on a routine basis and more often during high flows when sediment-transport rates were assumed to be the highest. Streamflow measurements were made according to standard techniques described by Rantz and others (1982). Conventional U.S. Series depth-integrating DH-48 (low flow) or D-49 (medium and high flow) samplers were used to collect periodic samples of suspended sediment at various points in the crosssection (Guy and Norman, 1970). The water-sediment mixture was poured into an 8-liter churn splitter which mixed the sample. A 350-ml aliquot was taken from the churn splitter and poured into a sediment bottle. The bottle containing the sample was then transported to the laboratory and stored in a cool, dry, dark place for laboratory determination of suspended-sediment concentration and particle-size distribution. At extremely low flow, only one sediment bottle was filled, so sample splitting was not necessary.

In addition, automatic pump samplers were installed at five of the ten stations. The automatic sampler is a portable device capable of collecting 24 separate, sequential water-sediment samples or a single composite sample. It can be used for sample collection at set time intervals or at equal flow volumes. The samplers installed in the study area were programmed to collect the water-sediment samples above low flow and at time intervals of 5, 10, 30 , and 60 minutes. The samplers utilized a peristaltic pump system to transport the sample from the stream to the sample bottle. The suction line was cleared before and after sampling. Once the 24 bottles were filled according to the desired time sequence, the samples were removed from the sampler casing and brought to the laboratory and stored for further 
analyses. The sampler casing was then filled with new empty bottles and programmed again for the next event. All samples were supplemented with samples collected by Survey personnel during storms.

Determination of suspended-sediment concentrations was made by either evaporation or filtration method. In the evaporation method, sediment was allowed to settle in the bottom of the sample bottle and the supernatant liquid was decanted. The sediment was washed into an evaporating dish, dried in an oven, and later weighed. Similar steps were followed in the filtration method except that instead of washing the sediment into an evaporating dish, the sediment was filtered through a glass-fiber filter (mesh size of 0.4 micrometers) in a crucible, and the crucible with the sediment was then oven dried.

The concentration of suspended-sediment is equal to the ratio of the dry weight of sediment to the volume of water-sediment mixture. This concentration is computed as a weight to weight ratio and is expressed in ppm (parts per million). A conversion factor is used to convert ppm to $\mathrm{mg} / \mathrm{L}$ (milligrams per liter) using the assumption that water density is equal to $1.000 \mathrm{~g} / \mathrm{mL}$ (gram per milliliter) plus or minus 0.005 , temperature is from $0^{\circ}$ to $29^{\circ} \mathrm{C}$, specific gravity of suspended-sediment is 2.65 , and the dissolved solids concentration is less than 10,000 parts per million (Guy, 1969). For suspended-sediment concentrations less than $15,900 \mathrm{ppm}$, the conversion factor is equal to unity (table 3 ).

Because suspended-sediment concentrations vary with water discharge, all values should be considered as instantaneous and representative only of the dis- charge at the given location and time. The data for each station are summarized in tables 4 to 13 . These data were also used to develop the plots shown in figures 4 to 13.

Samples of suspended sediment, obtained at a point by automatic pump samplers must be calibrated with depth-integrated samples to assure that they are representative. The standard technique of collecting simultaneous suspended-sediment samples by depth integration and by automatic sampler was not undertaken in this study. Percent differences in concentration therefore could not be obtained for comparison of instantaneous conditions. In lieu of these standard calibration techniques, a more generalized scheme was adopted.

For the five stations with automatic pump samplers, calibration was achieved by establishing individual sediment ratings (water discharge versus sediment load) for the depth-integrated and automatic-pump samples at each station (fig. 14). By checking trends of standardized residuals from the linear (log$\log$ ) relation, 2 to 3 linear segments were created to define the sediment rating for each sample type. Discharge ranges for each of the linear segments were determined by a procedure of moving averages. Regression equations were then fitted to each segment.

Calibration of the automatic-pump samples was then accomplished by calculating the difference between the 'true' sediment load (from the depth-integrated rating) and the load obtained from the automatic-pump rating, and by adding this difference to the original load determined for the pump sample

\begin{tabular}{|c|c|c|c|c|c|}
\hline \multicolumn{6}{|c|}{$\begin{array}{l}\text { concentration in milligrams per liter when used with } \\
\text { parts per million or the ravio (times } 10^{6} \text { ) of the } \\
\text { weight of sediment to the weight of the water-sediment } \\
\text { mixture (Guy, } 1960)\end{array}$} \\
\hline Rat io & $\mathrm{C}$ & Ratio & $\mathrm{C}$ & Ratio & $\mathrm{C}$ \\
\hline $0-15,900$ & 1.0 & $234,000-256,000$ & 1.18 & $417,000-434,000$ & 1.36 \\
\hline $16,000-47,000$ & 1.02 & $257,000-279,000$ & 1.20 & $435,000-451,000$ & 1.38 \\
\hline $48,000-76,000$ & 1.04 & $280,000-300,000$ & 1.22 & $452,000-467,000$ & 1.40 \\
\hline $77,000-105,000$ & 1.06 & $301,000-321,000$ & 1.24 & $468,000-483,000$ & 1.42 \\
\hline $106,000-132,000$ & 1.08 & $322,000-341,000$ & 1.26 & $484,000-498,000$ & 1.44 \\
\hline $133,000-159,000$ & 1.10 & $342,000-361,000$ & 1.28 & $499,000-513,000$ & 1.46 \\
\hline $160,000-184,000$ & 1.12 & $362,000-380,000$ & 1.30 & $514,000-528,000$ & 1.48 \\
\hline $185,000-209,000$ & 1.14 & $381,000-398,000$ & 1.32 & $529,000-542,000$ & 1.50 \\
\hline $210,000-233,000$ & 1.16 & $399,000-416,000$ & 1.34 & & \\
\hline
\end{tabular}


(fig. 14). A summary of the equations, discharge ranges, and number of samples is given in table 14.

It is understood that the calibration procedure used in this study is not conventional. However, sediment ratings for depth-integrated and automaticpump samples at 4 of the 5 stations are clearly different enough to provide confidence in the technique. Sediment rating of the fifth station (Río Turabo; station number 50053050) is matched closely, thereby requiring no calibration for the station.

Particle-size distribution of suspended sediment was determined by the sieve-pipet method (Guy, 1969). Sieves were used for sediment-particle sizes coarser than $0.062 \mathrm{~mm}$ (millimeters) and pipet was used for finer particle sizes. Sand was separated from the silt and clay by wet sieving with a 250-mesh (0.062 $\mathrm{mm})$.

The sand fraction was separated into grain sizes finer than $1.0,0.50,0.25,0.125$, and $0.062 \mathrm{~mm}$ by wet sieving because of the difficulty in removing the remaining clay from the sand particles by other methods. Material retained in the sieve was washed into an evaporating dish, dried, and weighed. The material passing through the sieve was poured with its wash water onto the next smaller-sized sieve. The process was repeated for each size category until the $0.062 \mathrm{~mm}$ sieve was used. Material passing through the $0.062 \mathrm{~mm}$ sieve was added to the material obtained during initial separation of fines from sands and processed by the pipet method. All samples were then dried and weighed after decanting and filtering.

Particle-size distribution of suspended-sediment was determined as percentage of dry mass. The dry weight of each fraction was divided by the total weight of the sample and expressed as a percentage. Computations and average percentages are summarized in table 15.

Instantaneous suspended-sediment discharge (the time rate at which a dry weight of sediment passes through a section of a stream), was computed using the instantaneous water discharge, sediment concentration, and a conversion factor. The formula used was (Potterfield, 1972):

$$
\text { Qs }=\text { QwCsk }
$$

where

$$
\begin{aligned}
& \text { Qs = suspended-sediment discharge rate, } \\
& \text { in tons per day (tons } / \mathrm{d}), \\
& \mathrm{Qw} \mathrm{=} \\
& \text { instantaneous water discharge rate, } \\
& \text { in cubic feet per second }\left(\mathrm{ft}^{3} / \mathrm{s}\right), \\
& \mathrm{Cs}=\text { suspended-sediment concentration, } \\
& \text { in milligrams per liter }(\mathrm{mg} / \mathrm{L}), \\
& \mathbf{k}=\text { conversion factor of } 0.0027
\end{aligned}
$$

The computed instantaneous sediment discharges for each station are summarized in tables 4 to 13 . These data, in conjunction with the instantaneous water discharge, are then used to develop suspendedsediment transport curves (ratings).

The ratio of instantaneous water discharge to average water discharge (Qi/Qa) was used as a general indicator of the magnitude of individual storm events. The ratio was computed by dividing the water discharge recorded at the time the suspended-sediment sample was collected, by the average water discharge computed for the period of record for each individual station (tables 4-13). Average water discharge for each stations is shown in table 16.

The specific conductance of the supernatant liquid and temperature were determined for each sample and the results are summarized (tables 4 to 13).

\section{RESULTS}

A total of 2,114 suspended-sediment samples were collected and analyzed during the investigation. Because of probable sampling errors, 10 samples were discarded after being analyzed. From the total samples collected, 7 percent (152 samples) were analyzed for particle-size distribution. Extreme values for the 1984-1986 period are listed in table 16. It should be noted that the maximum water discharge does not necessarily correspond to the maximum suspended-sediment concentration and load. 
Table 4. Suspended-sediment data for samples collected at station Rio Grande de Loiza at Quebrada Arenas

[US/CM, microsiemens per centimeter at 25 degree Celsius; CFS, cubic foot per second; MG/l, milligrams per liter;

$T / D A Y$, tons per day; Qi/Qa, ratio of instantaneous water discharge to average water discharge; .... missing data]

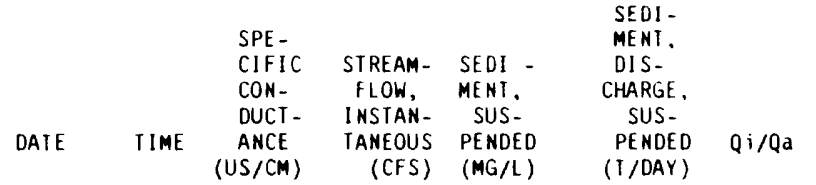

WATER YEAR DCTOBER 1983 TO SEPTEMBER 1984

\begin{tabular}{|c|c|c|c|c|c|c|c|}
\hline FEB & 14 & 1500 & $13 D$ & 28 & 338 & 26 & 0.90 \\
\hline FEB & 14 & 1600 & 130 & 28 & 425 & 32 & .90 \\
\hline MAR & 13 & 0945 & 152 & 9 & 0 & D & .29 \\
\hline MAR & 27 & 0915 & 163 & 7.2 & 1 & .02 & .23 \\
\hline APR & 11 & 0900 & 158 & 7.5 & 2 & .04 & .24 \\
\hline APR & 24 & 0915 & 162 & 6.5 & 1 & .02 & .21 \\
\hline MAY & 17 & 1015 & 167 & 4.8 & 4 & .05 & .15 \\
\hline AUG & 31 & 1540 & 149 & 15 & 50 & 2.0 & .48 \\
\hline SEPT & 12 & 1045 & 123 & 36 & 87 & 8.5 & .12 \\
\hline SEPT & 12 & 1100 & 123 & 34 & 96 & 8.8 & 1.1 \\
\hline SEPT & 12 & 1130 & 123 & 31 & 83 & .6 .9 & 1.0 \\
\hline SEPT & 14 & 1130 & 99 & 89 & 146 & 35 & 2.9 \\
\hline SEPI & 14 & 1135 & 99 & 79 & 137 & 29 & 2.5 \\
\hline SEPT & 14 & 1140 & 99 & 75 & 134 & 27 & 2.4 \\
\hline SEPT & 14 & 1145 & 98 & 72 & 129 & 25 & 2.3 \\
\hline SEPT & 14 & 1150 & 98 & 73 & 127 & 25 & 2.3 \\
\hline SEPT & 14 & 1155 & 98 & 73 & 139 & 27 & 2.3 \\
\hline SEPI & 14 & 1200 & 98 & 74 & 130 & 26 & 2.4 \\
\hline SEPI & 14 & 1205 & 98 & 72 & 124 & 24 & 2.3 \\
\hline SEPT & 14 & 1210 & 98 & 70 & 126 & 24 & 2.2 \\
\hline SEPT & 14 & 1215 & 98 & 68 & 146 & 27 & 2.2 \\
\hline SEPT & 18 & 1500 & 112 & 55 & 49 & 7.3 & 1.8 \\
\hline SEPT & 20 & 1230 & 89 & 114 & 167 & 51 & 3.7 \\
\hline SEPI & 20 & 1245 & 86 & 123 & 192 & 64 & 4.0 \\
\hline SEPT & 20 & 1300 & $B 6$ & 138 & 304 & 113 & 4.4 \\
\hline SEPT & 20 & 1315 & 85 & 160 & 453 & 196 & 5.1 \\
\hline SEPT & 20 & 1330 & 81 & 295 & 1,000 & 796 & 9.5 \\
\hline SEPT & 20 & 1340 & 81 & 298 & 924 & 743 & 9.6 \\
\hline SEPT & 20 & 1345 & 79 & 300 & 1,020 & B26 & 9.6 \\
\hline SEPT & 20 & 1350 & 79 & 305 & 1.040 & 856 & 9.8 \\
\hline SEPI & $2 D$ & 1355 & 71 & 309 & 1,060 & 884 & 9.9 \\
\hline SEPT & 20 & 1400 & 71 & 314 & 676 & 573 & 10 \\
\hline SEPI & $2 D$ & 1405 & 71 & 330 & 838 & 747 & 11 \\
\hline SEPT & 20 & 1410 & 71 & 346 & 692 & 646 & 11 \\
\hline SEPI & 20 & 1415 & 70 & 362 & 1.080 & 1,060 & 12 \\
\hline SEPT & 20 & 1420 & 70 & 413 & 813 & 907 & 13 \\
\hline SEPT & 20 & 1425 & 64 & 464 & 907 & 1,140 & 15 \\
\hline SEPI & 20 & 1430 & 62 & 515 & 1,090 & 1,520 & 17 \\
\hline SEPI & 20 & 1435 & 70 & 581 & 1,520 & 2,380 & 19 \\
\hline SEPI & 20 & 1440 & 56 & 650 & 1,000 & 1,760 & 21 \\
\hline SEP1 & 20 & 1445 & 58 & 717 & 865 & 1.670 & 23 \\
\hline SEPT & 20 & 1450 & 58 & 710 & 1.050 & 2.010 & 23 \\
\hline SEPT & 20 & 1455 & 58 & 702 & 1,150 & 2,180 & 23 \\
\hline SEPI & 20 & 1500 & 54 & 694 & 1,060 & 1.990 & 22 \\
\hline SEPI & 20 & 1505 & 54 & 626 & 1,160 & 1,960 & 20 \\
\hline SEPI & 20 & 1510 & 54 & 558 & 879 & 1,320 & 18 \\
\hline SEPT & 20 & 1515 & 54 & 490 & 911 & 1,210 & 16 \\
\hline
\end{tabular}

WATER YEAR OCTOBER 1984 TO SEPIEMBER 1985

$\begin{array}{lrrrrrrr}\text { OCT } & 25 & 1015 & 116 & 39 & 39 & 4.1 & 1.2 \\ \text { OCT } & 26 & 0945 & 120 & 43 & 46 & 5.3 & 1.4 \\ \text { OCT } & 29 & 1045 & -1 . & 23 & 4 & .25 & .74 \\ \text { DCT } & 31 & 0930 & 136 & 22 & 14 & .60 & .71 \\ \text { NOV } & 5 & 1345 & 43 & 525 & 1,290 & 1.820 & 17 \\ \text { NOV } & 7 & 1330 & 93 & 146 & 66 & 26 & 4.7 \\ \text { NOV } & 7 & 1453 & 94 & 165 & 168 & 75 & 5.3 \\ \text { NOV } & 9 & 1057 & 126 & 77 & 26 & 5.4 & 2.5 \\ \text { NOV } & 13 & 1315 & 140 & 43 & 18 & 2.1 & 1.4 \\ \text { NOV } & 14 & 1045 & 142 & 40 & 20 & 2.2 & 1.3 \\ & & & & & & & \\ \text { NOV } & 15 & 1020 & 134 & 44 & 20 & 2.4 & 1.4 \\ \text { NOV } & 20 & 1350 & 145 & 28 & 4 & .30 & .90 \\ \text { NOV } & 23 & 0930 & 143 & 31 & 11 & .92 & 1.0 \\ \text { NOV } & 26 & 1125 & 123 & 31 & 30 & 2.5 & 1.0 \\ \text { NOV } & 28 & 1015 & 150 & 23 & 12 & .75 & .74\end{array}$

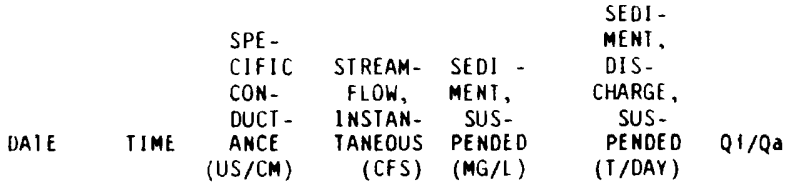

WATER YEAR OCTOBER 1984 IO SEPIEM8ER 1985

$\begin{array}{lrrrrrrr}\text { NOV } & 30 & 1015 & 148 & 21 & 3 & 0.17 & 0.68 \\ \text { DEC } & 3 & 1115 & 136 & 25 & 10 & .68 & .80 \\ \text { DEC } & 5 & 1040 & 152 & 20 & 6 & .32 & .64 \\ \text { DEC } & 10 & 1241 & 156 & 17 & 1 & .05 & .55 \\ \text { DEC } & 12 & 1000 & 158 & 17 & 2 & .09 & .55 \\ & & & & & & & \\ \text { DEC } & 13 & 1020 & 160 & 16 & 4 & .17 & .51 \\ \text { DEC } & 17 & 1200 & 147 & 15 & 3 & .12 & .48 \\ \text { DEC } & 19 & 1048 & 150 & 14 & 2 & .08 & .45 \\ \text { DEC } & 20 & 1219 & 150 & 14 & 0 & 0 & .45 \\ \text { DEC } & 26 & 0911 & 154 & 13 & 3 & .10 & .42 \\ & & & & & & & \\ \text { DEC } & 27 & 0935 & 158 & 12 & 4 & .13 & .39 \\ \text { JAN } & 8 & 1023 & 165 & 11 & 1 & .03 & .35 \\ \text { JAN } & 10 & 1050 & 160 & 10 & 2 & .06 & .32 \\ \text { JAN } & 15 & 1145 & 163 & 9.3 & 66 & 1.7 & .29 \\ \text { JAN } & 17 & 0945 & 157 & 9.0 & 1 & .02 & .29 \\ & & & & & & & \\ \text { JAN } & 18 & 0900 & 155 & 8.7 & 1 & .02 & .28 \\ \text { JAN } & 21 & 0930 & 158 & 8.4 & 2 & .05 & .28 \\ \text { JAN } & 25 & 0845 & 165 & 7.8 & 2 & .04 & .25 \\ \text { JAN } & 28 & 0915 & 162 & 8.4 & 14 & .31 & .27 \\ \text { JAN } & 30 & 1019 & 162 & 7.8 & 3 & .06 & .25\end{array}$

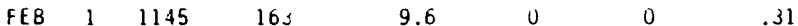

$\begin{array}{llllllll}\text { FEB } & 4 & 1020 & 163 & 9.6 & 0 & 0 & .31 \\ \text { FEB } & 6 & 0950 & 173 & 7.6 & 1 & .02 & .24 \\ \text { FEB } & 8 & 1040 & 170 & 8.8 & 2 & .04 & .25\end{array}$

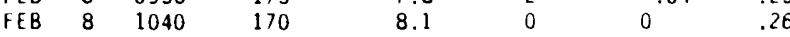

$\begin{array}{llllllll}\text { HEB } & 14 & 0915 & 170 & 8.1 & 2 & .04 & .26\end{array}$

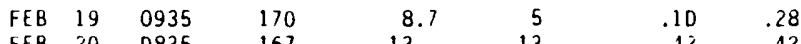

$\begin{array}{rrrrrrrr}\text { FEB } & 20 & 0835 & 167 & 13 & 13 & .10 & .28 \\ \text { FEB } & 22 & 1015 & 153 & 9.0 & 3 & .42 & .42 \\ \text { FEB } & 26 & 0925 & 153 & 9.9 & 14 & .35 & .32\end{array}$

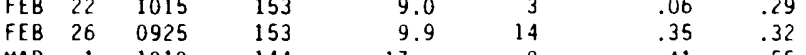

$\begin{array}{llllllll}\text { MAR } & 1 & 1010 & 144 & 17 & 9 & .41 & .55\end{array}$

$\begin{array}{llllllll}\text { MAR } & 5 & 1130 & 148 & 12 & 9 & .29 & .39\end{array}$

$\begin{array}{llllllll}\text { MAR } & 6 & 1205 & 1.58 & 14 & 73 & 2.8 & .45 \\ \text { MAR } & 7 & 1100 & 131 & 33 & 24 & 2.1 & 1.1\end{array}$

$\begin{array}{lllllll}\text { MAR } & 11 & 0900 & 160 & 15 & 2 & .08\end{array}$

$\begin{array}{llllllll}\text { MAR } & 13 & 1105 & 166 & 14 & 3 & .11 & .45\end{array}$

$\begin{array}{llllllll}\text { MAR } & 15 & 0900 & 163 & 13 & 3 & .10 & .42 \\ \text { MAR } & 19 & 0945 & 142 & 18 & 5 & .24 & .58\end{array}$

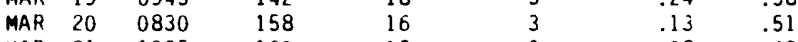

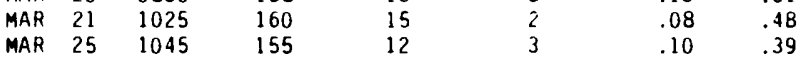

$\begin{array}{llllllll}\text { MAR } & 27 & 0921 & 160 & 12 & 2 & .06 & .39\end{array}$

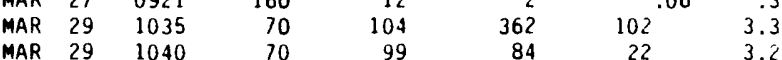

$\begin{array}{rrrrrrrr}\text { MAR } & 29 & 1040 & 70 & 99 & 84 & 22 & 3.2 \\ \text { APR } & 1 & 1010 & 145 & 12 & 4 & .13 & .39\end{array}$

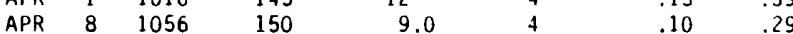

APR $10 \quad 0847$

150

APR $12 \quad 1030$

APR $26 \quad 1115$

$\begin{array}{lll}\text { MAY } & 14 & 1300 \\ \text { MAY } & 14 & 1315\end{array}$

8.1

9.

24

MAY $14 \quad 1330$

MAY $15 \quad 1915$

MAY 151930

MAY $15 \quad 1935$

MAY $15 \quad 1940$

MAY $15 \quad 1943$

MAY $15 \quad 2001$

MAY $15 \quad 2007$

MAY 152013

MAY $15 \quad 2017$

MAY $15 \quad 2021$

MAY $15 \quad 2028$

MAY $15 \quad 2035$

$\begin{array}{lll}\text { MAY } & 15 & 2041 \\ \text { MAY } & 15 & 2047\end{array}$ 


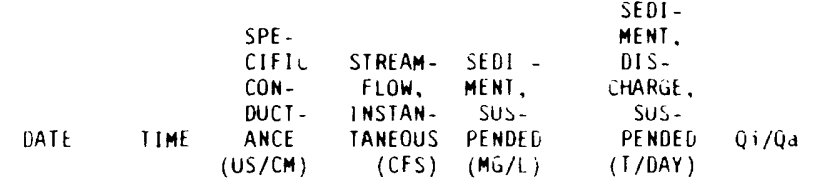

WAIER YEAR GCTOBER 1 y8̈ TO SEPTEMBER 1985 -Cont InUEd

MAY $15 \quad 2053$

MAY $15 \quad 2107$

MAY $15 \quad 211$

MAY $15 \quad 2122$

MAY $15 \quad 2130$

MAY $15 \quad 2140$

MAY $15 \quad 2150$

MAY $18 \quad 1400$

MAT $20 \quad 1345$

MAY $\angle Y \quad 1045$

MAY $29 \quad 1135$

JUNE S 1025

JUNE 7 1055

JUNE IO 1200

JUNE L $1 / 1145$

JUNE $14 \quad 1025$

JUNE $20 \quad 1055$

JUNE $24 \quad 1025$

JUNE $\angle 8 \quad 1010$

JULr 11145

Jule $5 \quad 1020$

Juler $8 \quad 1045$

JulY $15 \quad 1015$

JUIY $15 \quad 1030$

JULY $15 \quad 1045$

JuIY le 1215

Jut $r 22 \quad 0950$

JULY $29 \quad 1145$

AUG 10925

AUG $6 \quad 1020$

$\begin{array}{lrr}\text { AUG } & 9 & 0925 \\ \text { AUG } & 12 & 1025\end{array}$

AUG $19 \quad 1200$

AUG $23 \quad 1025$

AUG $30 \quad 1030$

SEPT $3 \quad 1110$

SEPT $6 \quad 1010$

$\begin{array}{lrr}\text { SEPI } & 9 & 1045 \\ \text { SEPI } 12 & 1815\end{array}$

$\begin{array}{lll}\text { SEPI } 12 & 1815 \\ \text { SEPT } 12 & 1820\end{array}$

SEPT $12 \quad 1825$

SEPI $12 \quad 1835$

SEPI $12 \quad 1845$

SEPI $12 \quad 1855$

SEPT $12 \quad 1905$

SEPT $12 \quad 1915$

SEPT $12 \quad 1930$

SEPI $12 \quad 1945$

SEPI $12 \quad 2000$

SEPT $12 \quad 2015$

SEPT $16 \quad 1240$

SEPT $23 \quad 1030$

SEPT $24 \quad 1325$

SEPT $25 \quad 0030$

SEPT $25 \quad 0035$

$\begin{array}{ll}56 & 59 \\ 55 & 497 \\ 50 & 46 \\ 56 & 42 \\ 56 & 37 \\ 75 & 332 \\ 55 & 32 \\ 55 & 309 \\ 68 & 28 \\ 136 & \\ 130 & \\ 236 & 17 \\ 131 & 1 \\ 120 & 14 \\ 135 & 1 \\ 144 & \\ 148 & \\ 150 & \\ 146 & \\ 147 & \end{array}$

595

595
497
467

427
375

$\begin{array}{lll}749 & 864 & 15 \\ 601 & 608 & 14\end{array}$

$601 \quad 608 \quad 14$

$32 \quad 668$

322

309
281

48
4849

19

14

14

$15 \quad 10$

$12 \quad 86$

9.3

9.0

11
9.3

8.7

52

115

115

90

125

130
148
145

145
1150

130

130
140
100

100
130

140

$140 \quad 1$

135

$90 \quad 903$

$80 \quad 1.060$

$75 \quad 1.210$

1.500

1.940
1.590

$$
1.150
$$

1.280

1.000

780
510

510
342
24

60
120

140
100

50

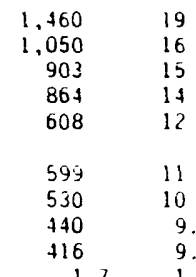

$1.7 \quad 9.0$

$\begin{array}{ll}.26 & .61\end{array}$

$.37 \quad .55$

$.11 \quad .45$

$.04 \quad .51$

$\begin{array}{cc}.40 & .48 \\ .10 & .39 \\ 3.2 & .45 \\ .48 & .32\end{array}$

$\begin{array}{ll}.48 & .32 \\ .42 & .30\end{array}$

$.15 \quad .2 y$

$\begin{array}{ll}.48 & .35 \\ .20 & .30 \\ .26 & .28\end{array}$

$16^{.26}$

1.7

$\begin{array}{rr}85 & 1.5 \\ 22 & 1.4 \\ 188 & 5.3 \\ 114 & 9.7\end{array}$

$\begin{array}{rr}114.17 & 4.7 \\ .51\end{array}$

$\begin{array}{ll}.30 & .71 \\ .14 & .42 \\ .14 & .42\end{array}$

$\begin{array}{ll}.14 & .12 \\ .12 & .35\end{array}$

$.03 \quad .35$

$\begin{array}{cc}.12 & .35 \\ .10 & .31 \\ +.6 & 2.0 \\ .15 & .58\end{array}$

$\begin{array}{ll}.15 & .58 \\ .08 & .15\end{array}$

$\begin{array}{ll}.19 & .35 \\ 2.250 & 29 \\ 4.190 & 34 \\ 4.320 & 39\end{array}$

1.320

1.600

$\begin{array}{rr}1.670 & 8.770 \\ 2.770 & 11.900\end{array}$

$2.480 \quad 7.700$

$1.990 \quad 6.8^{7} 0$

$\begin{array}{lll}1.700 & 4.590 \quad 32 \\ 1.180 & 2.190 & 25\end{array}$

37E $\quad 517$

$\begin{array}{rrr}376 & 517 & 16 \\ 521 & 482 & 11 \\ 5 & .32 & .7\end{array}$

14

67
368
685

1.000

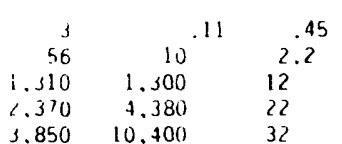

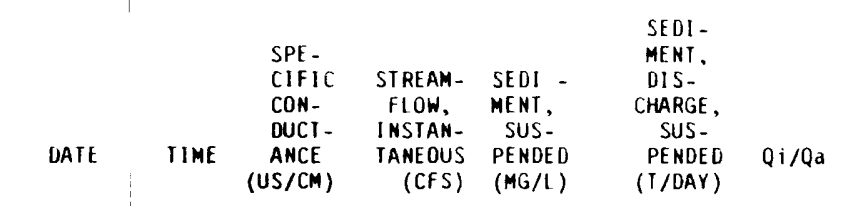

WAIER YEAR OCTOBER 1984 TD SEPTEMBER 1985

\begin{tabular}{lr|rrrrrr} 
SEPT & 25 & 0050 & 45 & 1,890 & 3,600 & 18,400 & 61 \\
SEPT & 25 & 0100 & 40 & 3,030 & 1,050 & 8,610 & 97 \\
SEPT & 25 & 0110 & 40 & 2,160 & 1,440 & 8,410 & 69 \\
SEPT & 25 & 0120 & 35 & 2,170 & 1,300 & 7,640 & 70 \\
SEPT & 25 & 0130 & 35 & 1,760 & 1,060 & 5,050 & 57 \\
& & & & & & & \\
SEPT & 25 & 0145 & 35 & 1,260 & 6,270 & 21,300 & 40 \\
SEPT & 25 & 0200 & 40 & 1,160 & 2,770 & 8,680 & 37 \\
SEPI 25 & 0215 & 50 & 1,370 & 375 & 1,390 & 44 \\
SEPT 25 & 0230 & 50 & 1,210 & 760 & 642 & 39 \\
SEPT 25 & 0245 & 40 & 977 & 663 & 194 & 31 \\
& & & & & & & \\
SEPT & 25 & 0300 & 50 & 663 & 202 & 363 & 21 \\
SEPT 25 & 0315 & 40 & 520 & 155 & 217 & 17
\end{tabular}

WAIER YEAR OCTOBER 1985 TO SEPIEMBER 1980

$\begin{array}{llll}\text { OCI } & 1 & 1515 \\ \text { OCI } & 1 & 1520 \\ \text { OCT } & 1 & 1525 \\ \text { OCT } & 1 & 1535 \\ \text { OCT } & 1 & 1545\end{array}$

\begin{tabular}{ll|l} 
OCT & 1 & 1555
\end{tabular}

\begin{tabular}{ll|l} 
OCI & 1 & 1605
\end{tabular}

\begin{tabular}{ll|l} 
OCT & 3 & 1230
\end{tabular}

$\begin{array}{lll}\text { OCT } & 6 & 0600 \\ \text { OCT } & 6 & 0605\end{array}$

$0 C 1 \quad 6 \quad 0610$

$0 \mathrm{Cl} \quad 6 \quad 0620$

OCT 60630

OCl 6,0650

OCT 6 6 10700

OCT 6 O 0715

OCT 6 OCI 0730

\begin{tabular}{ll|l} 
OCT & 6 & 0745 \\
OCT & 6 & 0800
\end{tabular}

OCT 6 6815

\begin{tabular}{ll|l} 
OCT & 6 & 0830
\end{tabular}

\begin{tabular}{ll|l}
$0 \mathrm{CT}$ & 6 & 0845 \\
\hline $\mathrm{CT}$ & 6 & 0900
\end{tabular}

\begin{tabular}{ll|l} 
OCT & 6 & 0900 \\
OCT & 6 & 0930
\end{tabular}

OCT $6 \quad 1000$

$\begin{array}{lll}\text { OCT } & 6 & 1030 \\ \text { OCT } & 7 & 1000\end{array}$

$\begin{array}{lll}\text { OCT } & 7 & 1005\end{array}$

OCT $15 \quad 1040$

70
70
70
70
70

$\begin{array}{rrrr}281 & 1,550 & 1,180 & 9 \\ 349 & 1,110 & 1,050 & 11 \\ 430 & 820 & 953 & 14\end{array}$

1,550

$1,180 \quad 9.0$

$481 \quad 740 \quad 961 \quad 15$

OCI 21 | 1000

\begin{tabular}{ll|l} 
OCT 24 & 0805
\end{tabular}

\begin{tabular}{ll|l} 
OCT & 24 & 0810
\end{tabular}

OCI $24 \quad 0815$

\begin{tabular}{ll|l} 
OCT & 24 & 0825
\end{tabular}

\begin{tabular}{ll|l} 
UL & 24 & 0815
\end{tabular}

0i1 $24 \quad 0845$

$0 C 1 \quad 24 \quad 0855$

UCI $24 \quad 1405$

OCI $26 \quad 1615$

OCT $26 \quad 1620$

OCT $26 \quad 1625$

OCI 26,1635

\begin{tabular}{ll|l} 
UC & 26 & 1645 \\
\hline$(1$ & 20 & 1655
\end{tabular}

\begin{tabular}{ll|l} 
u(.) it & 1.05
\end{tabular}

OCI $26 \quad 1715$

OCl $26 \quad 1730$

$0(1) 26 \quad 1745$

UĆ $26 \quad 1800$
$70 \quad 504$

$70 \quad 442$

$90 \quad 55$

40

534

622

747

891

887

810

733

853

1,260
1,370

1.330

1.230

1.230
1.150

1,150

2,990

2.910

283
279

33

$140 \quad 28$

140
80

$80 \quad 1.720$

$\begin{array}{ll}80 & 2,040\end{array}$

1.710

1,400

1.120

902

100
722

$65 \quad 865$

1.010

1.040

917
838

748
711
739
450

629
684

684
28

28
905

887

764

726

536

450

271

233

873

2,190
925

3,420

$\begin{array}{lll}72 & 2,660 \quad 43\end{array}$

$\begin{array}{lll}585 & 1.940 & 40\end{array}$

$\begin{array}{lll}145 & 481 & 40 \\ 240 & 744 & 37\end{array}$

$\begin{array}{lll}540 & 1.680 & 31\end{array}$

$\begin{array}{lll}4,200 & 33,900 & 96 \\ 2,280 & 17,900 & 94\end{array}$

$\begin{array}{lll}178 & 136 & 9.1\end{array}$

$\begin{array}{rrr}183 & 137 & 9.0 \\ 2 & .18 & 1.1\end{array}$

$\begin{array}{rrr}3.510 & 13.400 & 45 \\ 728 & 3.380 & 55\end{array}$

$158 \quad 1.350$

38

416

9.610

1,170

1,010

201

$7.510 \quad 17,500$

$6.000 \quad 16.400$

$4.430 \quad 12.400$

$3.030 \quad 8,000$

1.490

3. 300

$$
1.200
$$

2.310

$1.020 \quad 1.700$

$\begin{array}{rr}1.020 & 2.620 \\ 840 & 1.760\end{array}$

.90

9
.510
$13.400^{.68} \quad .90$

$\begin{array}{lll}376 & 2.070 & 66 \\ 517 & 2.390 & 55\end{array}$ 
Table 4. Suspended-sediment data for samples collected at station Rio Grande de Loiza at Quebrada Arenas

\begin{tabular}{|c|c|c|c|c|c|c|c|c|c|c|c|c|c|}
\hline DAIE & TIME & $\begin{array}{l}\text { SPE- } \\
\text { CIFIC } \\
\text { CON- } \\
\text { DUCI- } \\
\text { ANCE } \\
\text { (US/CM) }\end{array}$ & $\begin{array}{l}\text { SIREAM- } \\
\text { FLOW, } \\
\text { INSIAN- } \\
\text { TANEOUS } \\
\text { (CFS) }\end{array}$ & $\begin{array}{l}\text { SEDI - } \\
\text { MENT, } \\
\text { SUS- } \\
\text { PENDED } \\
\text { (MG/L) }\end{array}$ & $\begin{array}{l}\text { SEDI - } \\
\text { MENT, } \\
\text { DIS- } \\
\text { CHARGE, } \\
\text { SUS- } \\
\text { PENDED } \\
\text { (T/OAY) }\end{array}$ & $Q 1 / Q \mathrm{a}$ & DAIE & TIME & $\begin{array}{l}\text { SPE - } \\
\text { CIFIC } \\
\text { COH- } \\
\text { DUCT- } \\
\text { ANCE } \\
\text { (US/CM) }\end{array}$ & $\begin{array}{l}\text { STREAM- } \\
\text { FLOW, } \\
\text { INSTAN- } \\
\text { TANEOUS } \\
\text { (CFS) }\end{array}$ & $\begin{array}{l}\text { SEUI - } \\
\text { MENT. } \\
\text { SUS- } \\
\text { PENDEO } \\
\text { (MG/L) }\end{array}$ & $\begin{array}{l}\text { SEOI - } \\
\text { MENI. } \\
\text { OIS- } \\
\text { CHARGE. } \\
\text { SUS- } \\
\text { PENDED } \\
\text { (I/DAY) }\end{array}$ & $Q i / Q d$ \\
\hline
\end{tabular}

WATER YEAR OCTOBER 19B5 TO SEPTEMBER 1986-Cont inued

\begin{tabular}{|c|c|c|c|c|c|c|c|c|c|c|c|c|c|c|c|}
\hline $\begin{array}{l}O C T \\
O C T \\
O C T \\
O C T \\
\text { NOV }\end{array}$ & $\begin{array}{l}26 \\
26 \\
26 \\
31 \\
12\end{array}$ & $\begin{array}{l}1815 \\
1830 \\
1845 \\
1030 \\
1105\end{array}$ & $\begin{array}{r}50 \\
50 \\
50 \\
100 \\
70\end{array}$ & $\begin{array}{r}605 \\
357 \\
328 \\
57 \\
18\end{array}$ & $\begin{array}{r}816 \\
974 \\
737 \\
43 \\
4\end{array}$ & $\begin{array}{r}1,330 \\
939 \\
653 \\
6.6 \\
.19\end{array}$ & $\begin{array}{l}19 \\
11 \\
11 \\
1.8 \\
.58\end{array}$ & $\begin{array}{l}\text { MAR } \\
\text { MAR } \\
\text { MAR } \\
\text { APR } \\
\text { APR }\end{array}$ & $\begin{array}{l}20 \\
24 \\
31 \\
10 \\
14\end{array}$ & $\begin{array}{l}\text { OB30 } \\
0945 \\
1025 \\
0930 \\
1025\end{array}$ & $\begin{array}{l}170 \\
165 \\
130 \\
160 \\
160\end{array}$ & $\begin{array}{l}7.5 \\
7.2 \\
42 \\
13 \\
10\end{array}$ & $\begin{array}{r}1 \\
1 \\
24 \\
1 \\
1\end{array}$ & $\begin{array}{l}.02 \\
.02 \\
2.7 \\
.04 \\
.03\end{array}$ & $\begin{array}{r}.24 \\
.23 \\
1.4 \\
.42 \\
.32\end{array}$ \\
\hline $\begin{array}{l}\text { NOV } \\
\text { NOV } \\
\text { HOV } \\
\text { NOV } \\
\text { DEC }\end{array}$ & $\begin{array}{r}14 \\
18 \\
22 \\
27 \\
2\end{array}$ & $\begin{array}{l}1010 \\
1055 \\
0930 \\
1240 \\
1215\end{array}$ & $\begin{array}{r}140 \\
140 \\
90 \\
140 \\
150\end{array}$ & $\begin{array}{l}19 \\
50 \\
16 \\
12 \\
11\end{array}$ & $\begin{array}{r}6 \\
47 \\
5 \\
3 \\
8\end{array}$ & $\begin{array}{l}.31 \\
6.3 \\
.22 \\
.10 \\
.24\end{array}$ & $\begin{array}{l}.61 \\
1.6 \\
.51 \\
.39 \\
.35\end{array}$ & $\begin{array}{l}\text { APR } \\
\text { APR } \\
\text { MAY } \\
\text { MAY } \\
\text { MAY }\end{array}$ & $\begin{array}{r}21 \\
28 \\
5 \\
8 \\
22\end{array}$ & $\begin{array}{l}1020 \\
1215 \\
1015 \\
11.00 \\
0935\end{array}$ & $\begin{array}{l}180 \\
160 \\
150 \\
100 \\
160\end{array}$ & $\begin{array}{c}9.1 \\
12 \\
13 \\
17 \\
109\end{array}$ & $\begin{array}{r}1 \\
2 \\
4 \\
8 \\
454\end{array}$ & $\begin{array}{r}.02 \\
.06 \\
.14 \\
.37\end{array}$ & $\begin{array}{l}.29 \\
.39 \\
.42 \\
.55 \\
3.5\end{array}$ \\
\hline $\begin{array}{l}\text { UEC } \\
\text { DEC } \\
\text { DEC } \\
\text { DEC } \\
\text { DEC }\end{array}$ & $\begin{array}{r}6 \\
9 \\
12 \\
16 \\
23\end{array}$ & $\begin{array}{l}1035 \\
1040 \\
1125 \\
1110 \\
0940\end{array}$ & $\begin{array}{l}150 \\
150 \\
140 \\
145 \\
150\end{array}$ & $\begin{array}{c}11 \\
9.9 \\
18 \\
9.3 \\
9.3\end{array}$ & $\begin{array}{r}4 \\
17 \\
6 \\
3 \\
5\end{array}$ & $\begin{array}{l}.12 \\
.45 \\
.29 \\
.08 \\
.13\end{array}$ & $\begin{array}{l}.35 \\
.32 \\
.58 \\
.30 \\
.30\end{array}$ & $\begin{array}{l}\text { MAY } \\
\text { JUNE } \\
\text { JUNE } \\
\text { JUNE } \\
\text { JULY }\end{array}$ & $\begin{array}{r}22 \\
2 \\
18 \\
23 \\
1\end{array}$ & $\begin{array}{l}0950 \\
1215 \\
1000 \\
1035 \\
1025\end{array}$ & $\begin{array}{l}120 \\
165 \\
160 \\
170 \\
160\end{array}$ & $\begin{array}{l}99 \\
32 \\
19 \\
16 \\
19\end{array}$ & $\begin{array}{r}393 \\
5 \\
6 \\
2 \\
2\end{array}$ & $\begin{array}{r}105 \\
.43 \\
.31 \\
.09 \\
.10\end{array}$ & $\begin{array}{l}3.2 \\
1.0 \\
.61 \\
.51 \\
.61\end{array}$ \\
\hline $\begin{array}{l}\text { OEC } \\
\text { JAN } \\
\text { JAN } \\
\text { JAN } \\
\text { JAN }\end{array}$ & $\begin{array}{r}27 \\
8 \\
13 \\
16 \\
21\end{array}$ & $\begin{array}{l}0945 \\
0925 \\
1200 \\
1150 \\
1050\end{array}$ & $\begin{array}{l}145 \\
150 \\
150 \\
150 \\
145\end{array}$ & $\begin{array}{l}9.0 \\
7.1 \\
8.1 \\
7.2 \\
22\end{array}$ & $\begin{array}{r}5 \\
9 \\
5 \\
7 \\
91\end{array}$ & $\begin{array}{l}.12 \\
.17 \\
.11 \\
.14 \\
5.4\end{array}$ & $\begin{array}{l}.29 \\
.23 \\
.26 \\
.23 \\
.71\end{array}$ & $\begin{array}{l}\text { JULY } \\
\text { JULY } \\
\text { JULY } \\
\text { AUG } \\
\text { AUG }\end{array}$ & $\begin{array}{r}7 \\
14 \\
29 \\
4 \\
12\end{array}$ & $\begin{array}{l}1200 \\
1230 \\
1025 \\
1115 \\
1145\end{array}$ & $\begin{array}{l}155 \\
170 \\
190 \\
170 \\
150\end{array}$ & $\begin{array}{l}17 \\
13 \\
9.4 \\
11 \\
12\end{array}$ & $\begin{array}{r}32 \\
3 \\
4 \\
4 \\
20\end{array}$ & $\begin{array}{l}1.5 \\
.11 \\
.10 \\
.12 \\
.65\end{array}$ & $\begin{array}{l}.55 \\
.41 \\
.30 \\
.35 \\
.35\end{array}$ \\
\hline $\begin{array}{l}\text { JAN } \\
\text { JAN } \\
\text { FEB } \\
\text { FEB } \\
\text { FEB }\end{array}$ & $\begin{array}{r}27 \\
30 \\
3 \\
10 \\
13\end{array}$ & $\begin{array}{l}1120 \\
1140 \\
1000 \\
0945 \\
1245\end{array}$ & $\begin{array}{l}145 \\
150 \\
150 \\
160 \\
155\end{array}$ & $\begin{array}{l}7.2 \\
7.0 \\
6.3 \\
5.8 \\
8.5\end{array}$ & $\begin{array}{l}2 \\
1 \\
5 \\
1 \\
1\end{array}$ & $\begin{array}{l}.04 \\
.02 \\
.08 \\
.02 \\
.02\end{array}$ & $\begin{array}{l}.23 \\
.22 \\
.20 \\
.19 \\
.27\end{array}$ & $\begin{array}{l}\text { AUG } \\
\text { AUG } \\
\text { AUG } \\
\text { SEPT } \\
\text { SEPT }\end{array}$ & $\begin{array}{r}19 \\
26 \\
29 \\
2 \\
8\end{array}$ & $\begin{array}{l}1000 \\
1030 \\
1030 \\
1130 \\
1040\end{array}$ & $\begin{array}{l}170 \\
\ldots \\
\ldots \\
150\end{array}$ & $\begin{array}{c}8.0 \\
8.0 \\
126 \\
13 \\
9.7\end{array}$ & $\begin{array}{r}5 \\
3 \\
110 \\
3 \\
7\end{array}$ & $37 \begin{array}{r}.13 \\
.06 \\
.11 \\
.18\end{array}$ & $\begin{array}{r}.25 \\
.25 \\
4.0 \\
.41 \\
.31\end{array}$ \\
\hline $\begin{array}{l}\text { FEB } \\
\text { FEB } \\
\text { FEB } \\
\text { MAR } \\
\text { MAR }\end{array}$ & $\begin{array}{r}18 \\
24 \\
27 \\
3 \\
17\end{array}$ & $\begin{array}{l}1210 \\
0910 \\
0925 \\
1415 \\
1040\end{array}$ & $\begin{array}{l}150 \\
160 \\
170 \\
170 \\
170\end{array}$ & $\begin{array}{l}8.5 \\
8.8 \\
8.7 \\
8.0 \\
8.3\end{array}$ & $\begin{array}{l}3 \\
1 \\
4 \\
1 \\
7\end{array}$ & $\begin{array}{l}.07 \\
.02 \\
.09 \\
.02 \\
.16\end{array}$ & $\begin{array}{l}.27 \\
.28 \\
.28 \\
.26 \\
.27\end{array}$ & $\begin{array}{l}\text { SEPT } \\
\text { SEPT } \\
\text { SEPT } \\
\text { SEPT } \\
\text { SEPT }\end{array}$ & $\begin{array}{l}15 \\
19 \\
22 \\
26 \\
29\end{array}$ & $\begin{array}{l}1150 \\
0920 \\
1235 \\
0945 \\
1055\end{array}$ & $\begin{array}{l}175 \\
160 \\
150 \\
120 \\
145\end{array}$ & $\begin{array}{l}8.0 \\
6.7 \\
12 \\
23 \\
10\end{array}$ & $\begin{array}{r}13 \\
7 \\
9 \\
13 \\
8\end{array}$ & $\begin{array}{l}.28 \\
.13 \\
.29 \\
.81 \\
.22\end{array}$ & $\begin{array}{l}.25 \\
.21 \\
.38 \\
.41 \\
.25\end{array}$ \\
\hline
\end{tabular}

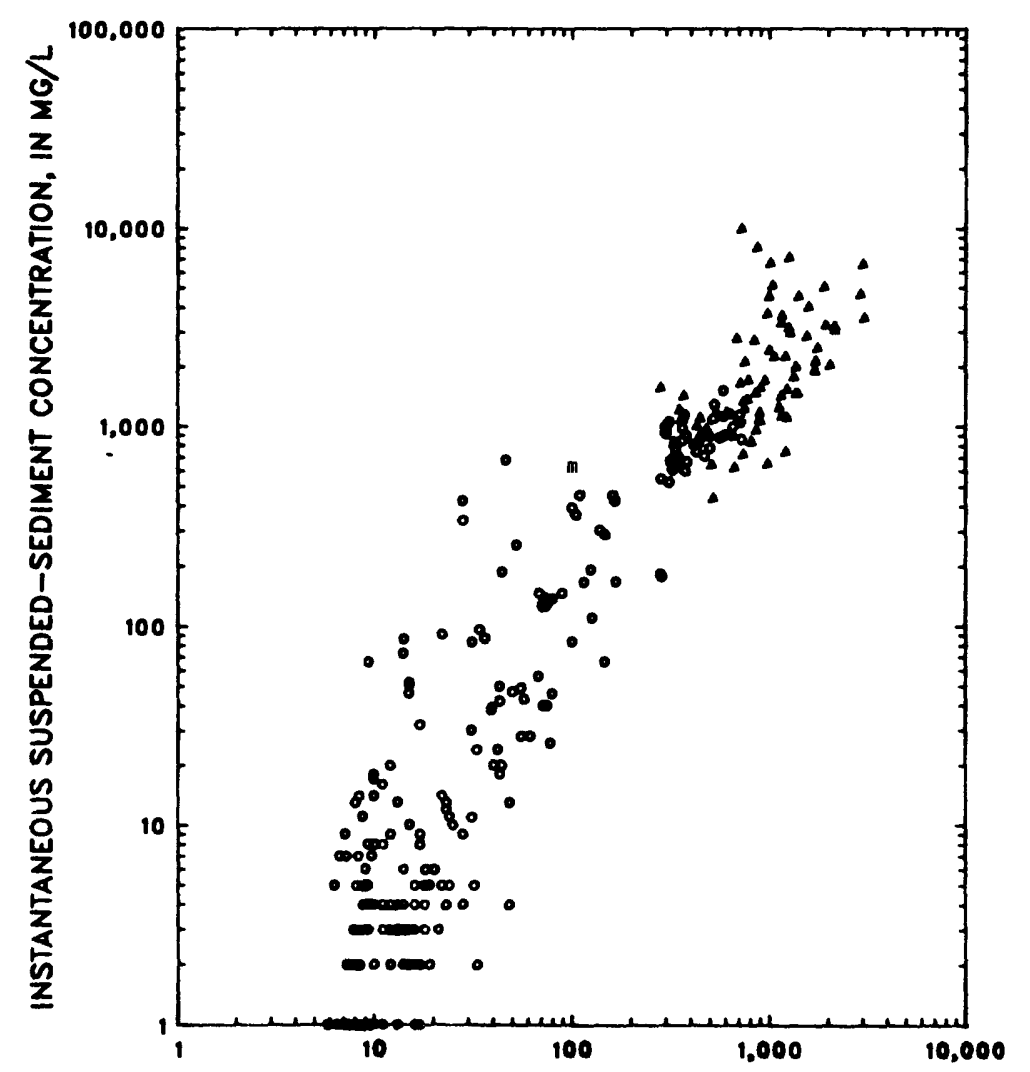

EXPLANATION

- field sample

- isco SAMPLE

INSTANTANEOUS WATER DISCHARGE, IN CUBIC. FEET PER SECOND

Figure 4.--Relation of suspended-sediment concentration to stream discharge

for station Río Grande de Loíza at Quebrada Arenas. 


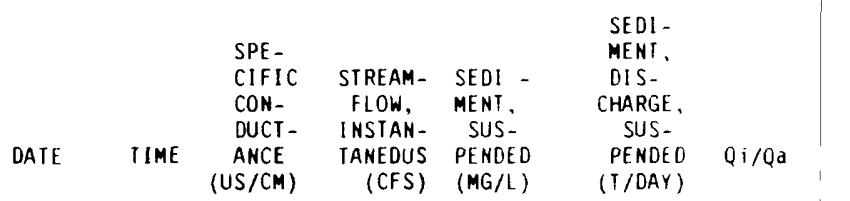

WATER YEAR OCTOBER 1983 TO SEPTEMBER 1984

\begin{tabular}{|c|c|c|c|c|c|c|c|}
\hline $\begin{array}{l}\text { SEPT } \\
\text { SEPT } \\
\text { SEPT } \\
\text { SEPT } \\
\text { SEPT }\end{array}$ & $\begin{array}{r}5 \\
5 \\
5 \\
5 \\
11\end{array}$ & $\begin{array}{l}1500 \\
1503 \\
1510 \\
1515 \\
1500\end{array}$ & $\begin{array}{r}90 \\
94 \\
117 \\
120 \\
263\end{array}$ & $\begin{array}{r}\mathrm{e} 100 \\
\mathrm{e} 100 \\
\mathrm{e} 95 \\
\mathrm{e} 95 \\
1.9\end{array}$ & $\begin{array}{r}50,400 \\
43,100 \\
37,500 \\
37,100 \\
84\end{array}$ & $\begin{array}{r}13,600 \\
11,100 \\
9,620 \\
9,500 \\
.43\end{array}$ & $\begin{array}{c}10 \\
10 \\
9.7 \\
9.7 \\
.20\end{array}$ \\
\hline $\begin{array}{l}\text { SEPT } \\
\text { SEPT } \\
\text { SEPT } \\
\text { SEPI } \\
\text { SEPI }\end{array}$ & $\begin{array}{l}12 \\
12 \\
12 \\
13 \\
13\end{array}$ & $\begin{array}{l}1200 \\
1215 \\
1245 \\
1430 \\
1432\end{array}$ & $\begin{array}{r}256 \\
257 \\
260 \\
84 \\
93\end{array}$ & $\begin{array}{r}3.6 \\
3.6 \\
3.4 \\
101 \\
94\end{array}$ & $\begin{array}{r}294 \\
298 \\
256 \\
52,400 \\
56,100\end{array}$ & $\begin{array}{r}2.8 \\
2.9 \\
2.4 \\
14,300 \\
14,200\end{array}$ & $\begin{array}{l}.39 \\
11.39 \\
10\end{array}$ \\
\hline $\begin{array}{l}\text { SEPT } \\
\text { SEPT } \\
\text { SEPT } \\
\text { SEPT } \\
\text { SEPT }\end{array}$ & $\begin{array}{l}13 \\
13 \\
13 \\
13 \\
13\end{array}$ & $\begin{array}{l}1435 \\
1439 \\
1440 \\
1442 \\
1445\end{array}$ & $\begin{array}{r}85 \\
90 \\
101 \\
105 \\
125\end{array}$ & $\begin{array}{l}83 \\
68 \\
64 \\
57 \\
46\end{array}$ & $\begin{array}{l}40,200 \\
37,900 \\
34,300 \\
31,500 \\
25,100\end{array}$ & $\begin{array}{l}8,990 \\
6,960 \\
5,930 \\
4,850 \\
3,120\end{array}$ & $\begin{array}{l}8.9 \\
7.3 \\
6.9 \\
6.1 \\
4.9\end{array}$ \\
\hline $\begin{array}{l}\text { SEPT } \\
\text { SEPT } \\
\text { SEPT } \\
\text { SEP } \\
\text { SEPI }\end{array}$ & $\begin{array}{l}13 \\
13 \\
13 \\
13 \\
13\end{array}$ & $\begin{array}{l}1450 \\
1455 \\
1500 \\
1505 \\
1510\end{array}$ & $\begin{array}{l}131 \\
142 \\
170 \\
160 \\
162\end{array}$ & $\begin{array}{l}52 \\
59 \\
65 \\
61 \\
58\end{array}$ & $\begin{array}{r}17,300 \\
16,600 \\
13,000 \\
8,980 \\
6,140\end{array}$ & $\begin{array}{r}2,430 \\
2,650 \\
2,320 \\
1,430 \\
962\end{array}$ & $\begin{array}{l}5.6 \\
6.3 \\
7.0 \\
6.5 \\
6.2\end{array}$ \\
\hline $\begin{array}{l}\text { SEPT } \\
\text { SEPT } \\
\text { SEPT } \\
\text { SEPI } \\
\text { SEPT } \\
\text { SEPT }\end{array}$ & $\begin{array}{l}13 \\
13 \\
13 \\
13 \\
13 \\
19\end{array}$ & $\begin{array}{l}1515 \\
1520 \\
1525 \\
1530 \\
1535 \\
1030\end{array}$ & $\begin{array}{l}165 \\
165 \\
158 \\
158 \\
150 \\
170\end{array}$ & $\begin{array}{l}54 \\
57 \\
60 \\
63 \\
59 \\
37\end{array}$ & $\begin{array}{r}5,910 \\
5,970 \\
6,450 \\
5,230 \\
4,510 \\
499\end{array}$ & $\begin{array}{r}862 \\
919 \\
1.040 \\
890 \\
718 \\
50\end{array}$ & $\begin{array}{l}5.6 \\
6.1 \\
6.4 \\
6.8 \\
6.3 \\
4.0\end{array}$ \\
\hline
\end{tabular}

WAIER YEAR OCTOBER 1984 TO SEPIEMEER 1985

$\begin{array}{lrrrrrrr}\text { UCT } & 25 & 0945 & 217 & 4.7 & 305 & 3.9 & .50 \\ \text { OCT } & 29 & 1000 & -1 . & 2.3 & 8 & .05 & .25 \\ \text { NOV } & 5 & 1300 & 60 & 318 & 2.070 & 1.780 & 34 \\ \text { NOV } & 7 & 1535 & 112 & 126 & 648 & 220 & 14 \\ \text { NOV } & 7 & 1545 & 112 & 124 & 668 & 224 & 13 \\ & & & & & & & \\ \text { NOV } & 13 & 1220 & 20 & 8 & 20 & .43 & .86 \\ \text { NOV } & 20 & 1220 & 253 & 6.2 & 3 & .05 & .66 \\ \text { DEC } & 5 & 1000 & 258 & 4.3 & 1 & .01 & .46 \\ \text { DEC } & 17 & 1140 & 284 & 3.0 & 8 & .06 & .32 \\ \text { DEC } & 20 & 1119 & 266 & 1.7 & 2 & .01 & .18 \\ \text { JAN } & 10 & 1130 & 278 & 1.4 & 3 & .01 & .15 \\ \text { JAN } & 23 & 0943 & 276 & 1.1 & 1 & 0 & .12 \\ \text { FEB } & 1 & 1102 & 293 & 1.1 & 5 & .01 & .12 \\ \text { FEB } & 8 & 1005 & 309 & .88 & 4 & .01 & .09 \\ \text { FEB } & 22 & 0935 & 287 & .94 & 9 & .02 & .10 \\ & & & & & & & \\ \text { MAR } & 7 & 1023 & 270 & 2.1 & 465 & 2.6 & .22 \\ \text { MAR } & 13 & 0945 & 291 & .94 & 9 & .02 & .10 \\ \text { MAR } & 21 & 0946 & 295 & .88 & 12 & .03 & .09 \\ \text { APR } & 12 & 1000 & 270 & .83 & 3 & .01 & .09 \\ \text { MAY } & 14 & 1130 & 151 & 12 & 2 & .06 & 1.3 \\ & & & & & & \\ \text { MAY } & 14 & 1145 & 152 & 13 & 2 & .06 & 1.4 \\ \text { MAY } & 14 & 1200 & 154 & 11 & 162 & 4.8 & 1.2 \\ \text { MAY } & 15 & 1835 & 102 & 96 & 695 & 180 & 10 \\ \text { MAY } & 15 & 1845 & 106 & 87 & 406 & 95 & 9.3 \\ \text { MAY } & 15 & 1850 & 107 & 86 & 592 & 137 & 9.3 \\ \text { MAY } & 15 & 1900 & 105 & 83 & 924 & 207 & 8.9 \\ \text { MAY } & 15 & 1905 & 104 & 85 & 834 & 192 & 9.1 \\ \text { MAY } & 15 & 1910 & 100 & 88 & 1.040 & 247 & 9.4 \\ \text { MAY } 15 & 1915 & 100 & 90 & 2.040 & 490 & 9.6 \\ \text { MAY } & 15 & 1920 & 94 & 103 & 1.840 & 512 & 11 \\ \text { MAY } & 15 & 1925 & 94 & 116 & 4.250 & 1.330 & 12 \\ \text { MAY } & 15 & 1930 & 100 & 128 & 1,120 & 387 & 14 \\ \text { MAY } & 15 & 1935 & 94 & 138 & 874 & 326 & 15 \\ \text { MAY } & 15 & 1950 & 90 & 156 & 1.100 & 463 & 17 \\ \text { MAY } & 15 & 2005 & 90 & 149 & 800 & 321 & 16\end{array}$

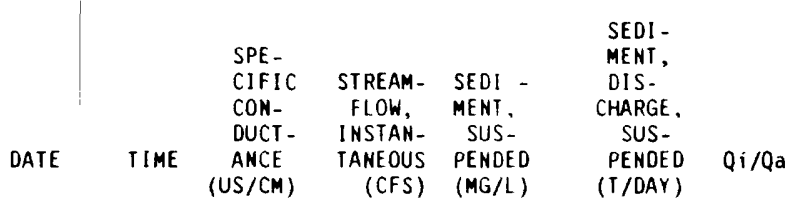

WATER YEAR OCTOBER 1984 TO SEPTEMBER 19BS

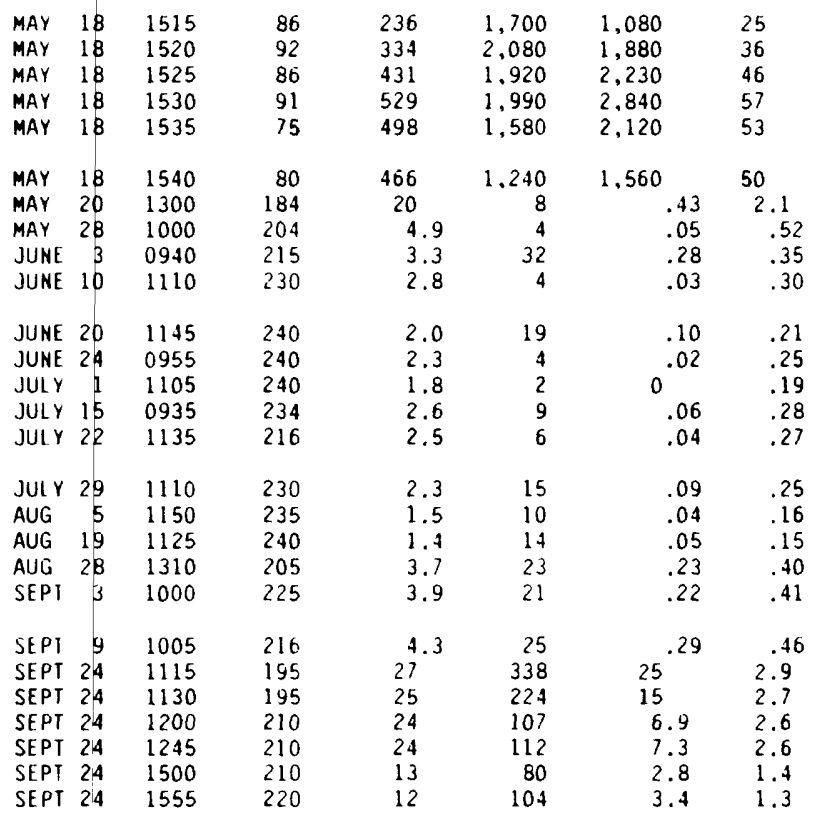

WATER YEAR OCIOBER 1985 IO SEPTEMBER 1986

\begin{tabular}{|c|c|c|c|c|c|c|c|}
\hline OLT & 7 & 1130 & 100 & 173 & 262 & 122 & 19 \\
\hline$O C T$ & 7 & 1125 & 100 & 171 & 282 & 130 & 18 \\
\hline $\mathrm{OCI}$ & 7 & 1420 & 110 & 109 & 64 & 19 & 12 \\
\hline $\mathrm{OCl}$ & 7 & 1430 & 120 & 106 & 105 & 30 & 11 \\
\hline $\mathrm{OCT}$ & 31 & 0940 & 130 & 45 & 72 & 8.7 & 4.8 \\
\hline NOV & 12 & 1030 & 200 & 17 & 530 & 24 & 1.8 \\
\hline NOV & 18 & 1020 & 130 & 31 & 33 & 2.8 & 3.3 \\
\hline $\mathrm{DEC}$ & 2 & 1325 & 250 & 4.7 & 1 & .01 & .50 \\
\hline$D E C$ & 9 & 1005 & 270 & 4.5 & 1 & .01 & .48 \\
\hline $\mathrm{DEC}$ & 16 & 1035 & 250 & 3.9 & 1 & .01 & .42 \\
\hline $\mathrm{DEC}$ & 23 & 0910 & 270 & 3.9 & 1 & .01 & .42 \\
\hline JAN & 13 & 1120 & 260 & 3.1 & 1 & .01 & .33 \\
\hline JAN & 21 & 1020 & 260 & 3.3 & 1 & .01 & .35 \\
\hline JAN & 27 & 1140 & 260 & 2.2 & 1 & .01 & .24 \\
\hline FEB & 3 & 0925 & 275 & 3.4 & 1 & .01 & .36 \\
\hline FEB & 18 & 1235 & 260 & 3.0 & 1 & .01 & .32 \\
\hline FEB & 26 & 0855 & 280 & 2.2 & $i$ & .01 & .24 \\
\hline MAR & 17 & 1010 & 290 & 1.7 & 3 & .01 & .18 \\
\hline MAR & 24 & 0915 & 290 & 1.5 & 1 & .01 & .16 \\
\hline MAR & 31 & 0950 & 270 & 2.1 & 6 & .01 & .23 \\
\hline$A P R$ & 14 & 0940 & 290 & 1.5 & 1 & .01 & .16 \\
\hline APR & 21 & 0950 & 300 & 1.7 & 2 & .01 & .18 \\
\hline MAY & 1 & 1250 & 180 & 43 & 530 & 62 & 4.6 \\
\hline MAY & 1 & 1300 & 160 & 34 & 429 & 39 & 3.6 \\
\hline MAY & 1 & 1320 & 140 & 24 & 355 & 23 & 2.6 \\
\hline MAY & 19 & 1330 & 190 & 5.3 & 4 & .06 & .57 \\
\hline JUNE & 2 & 1240 & 225 & 2.8 & 4 & .03 & .30 \\
\hline JUNE & 9 & 0940 & 220 & 5.3 & 199 & 2.8 & .57 \\
\hline JUNE & 9 & 1000 & 210 & 5.1 & 173 & 2.4 & .55 \\
\hline JUNE & 23 & 0955 & 280 & 1.9 & 3 & .02 & .20 \\
\hline$J U L Y$ & 1 & 1000 & 290 & 1.8 & 3 & .02 & .19 \\
\hline JUIY & 14 & 1255 & 260 & 3.6 & 24 & $.2 \hat{3}$ & .39 \\
\hline AUG & 4 & 1025 & 300 & 1.8 & 1 & 0 & .19 \\
\hline AUG & 19 & 0900 & 300 & 1.8 & 2 & .01 & .19 \\
\hline AUG & 29 & 1000 & 130 & 39 & 132 & 14 & 4.2 \\
\hline SEPI & 8 & 0955 & 255 & 1.4 & 0 & 0 & .15 \\
\hline SEPT & 29 & 1010 & 230 & 1.9 & 1 & .01 & .20 \\
\hline
\end{tabular}




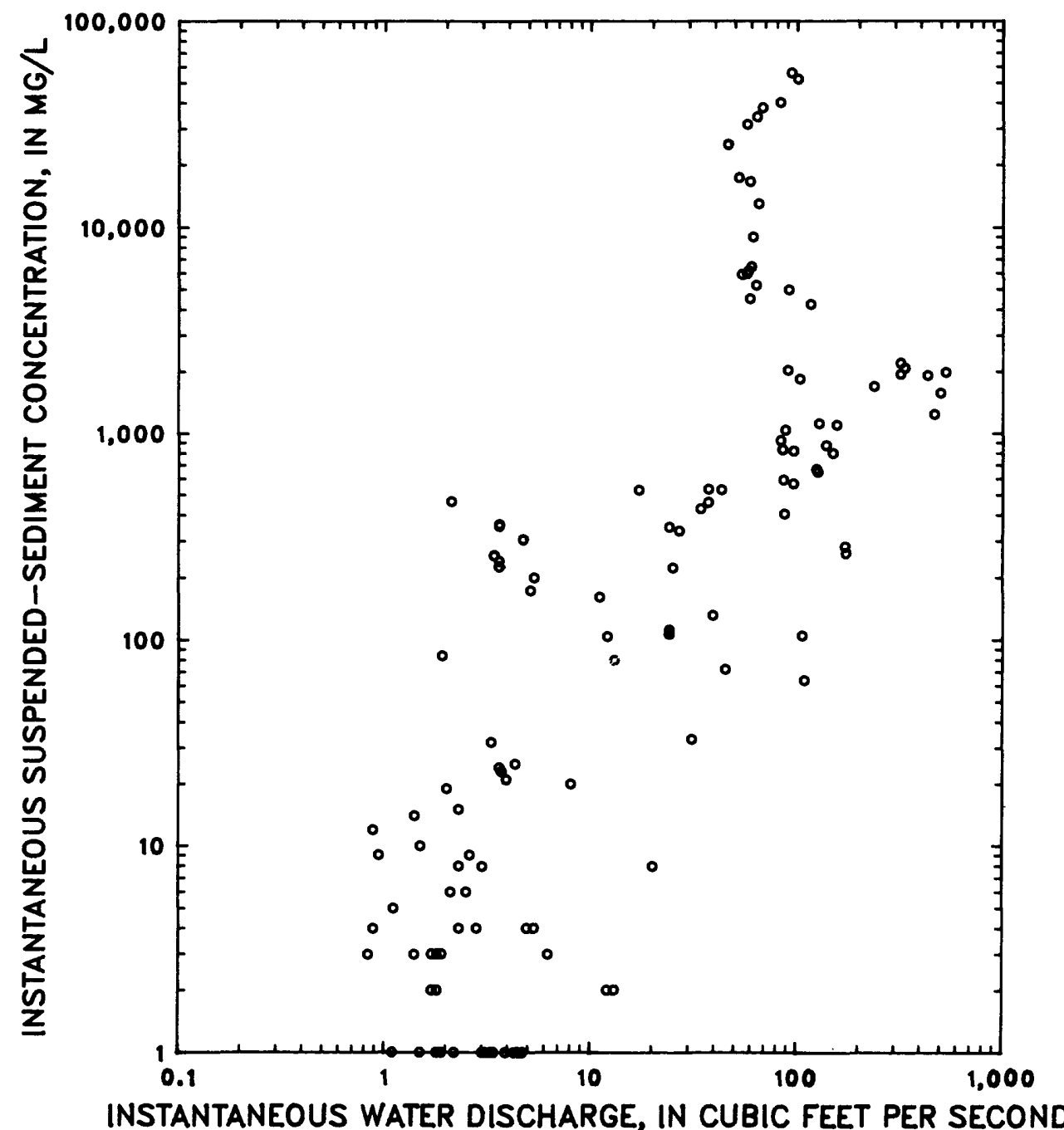

Figure 5.--Relation of suspended-sediment concentration to stream discharge for station Quebrada Blanca at Jagual. 
Iable 6. Suspended-sediment data for samples collected at station Quebrada Salvatierra near San Lorenzo

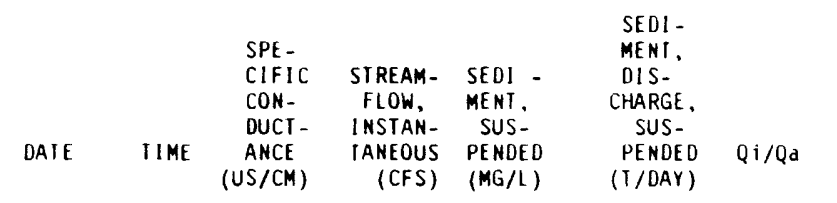

WATER YEAR OCIOBER 1983 TO SEPTEMBER 1984

\begin{tabular}{|c|c|c|c|c|c|c|c|}
\hline$A P K$ & 24 & 1015 & 442 & .93 & 1 & 0 & 0.12 \\
\hline MAY & 17 & 1130 & 414 & 1.2 & 5 & .02 & .15 \\
\hline MAY & 24 & 1108 & -- & 1.1 & 21 & .06 & .14 \\
\hline MAY & 25 & 1115 & $\ldots$ & 1.6 & 18 & .08 & .20 \\
\hline MAY & 30 & 1101 & 259 & 16 & 74 & 3.2 & 2.0 \\
\hline MAY & 30 & 1105 & 254 & 16 & 74 & 3.2 & $\angle .0$ \\
\hline MAY & 30 & 1110 & 259 & 16 & 77 & 3.3 & 2.0 \\
\hline JUNE & 7 & 1242 & 265 & 9.4 & 42 & 1.1 & 1.2 \\
\hline JUNE & 28 & 1240 & 354 & 1.4 & 0 & 0 & .18 \\
\hline JULY & 5 & 1420 & 188 & 38 & 71 & 7.1 & 4.8 \\
\hline JULY & 5 & 1453 & 188 & 34 & 66 & 6.1 & 4.3 \\
\hline AUG & 27 & 1115 & 318 & 4.2 & 4 & .05 & .53 \\
\hline SEPI & 12 & 0955 & 318 & 12 & 116 & 3.8 & 1.5 \\
\hline SEPT & 12 & 1030 & 313 & 10 & 85 & 2.3 & 1.3 \\
\hline SEPI & 12 & 1100 & 319 & 8.5 & 82 & 1.9 & 1.1 \\
\hline SEPT & 14 & 1110 & 168 & 112 & 223 & 67 & 14 \\
\hline SEPT & 14 & 1135 & 170 & 102 & 198 & 54 & 13 \\
\hline SEPT & 14 & 1205 & 175 & 93 & 190 & 48 & 12 \\
\hline SEPT & 14 & 1235 & 176 & 85 & 170 & 38 & 11 \\
\hline SEPT & 14 & 1305 & 193 & 79 & 148 & 32 & 10 \\
\hline SEPT & 17 & 1513 & 253 & 20 & 81 & 4.4 & 2.5 \\
\hline SEPT & 19 & 1100 & 223 & 30 & 67 & 5.4 & 3.8 \\
\hline SEPI & 19 & 1115 & 228 & 31 & 72 & 6.0 & 3.8 \\
\hline
\end{tabular}

WATER YEAR OCIOBER 1984 TO SEPIEMBER 1985

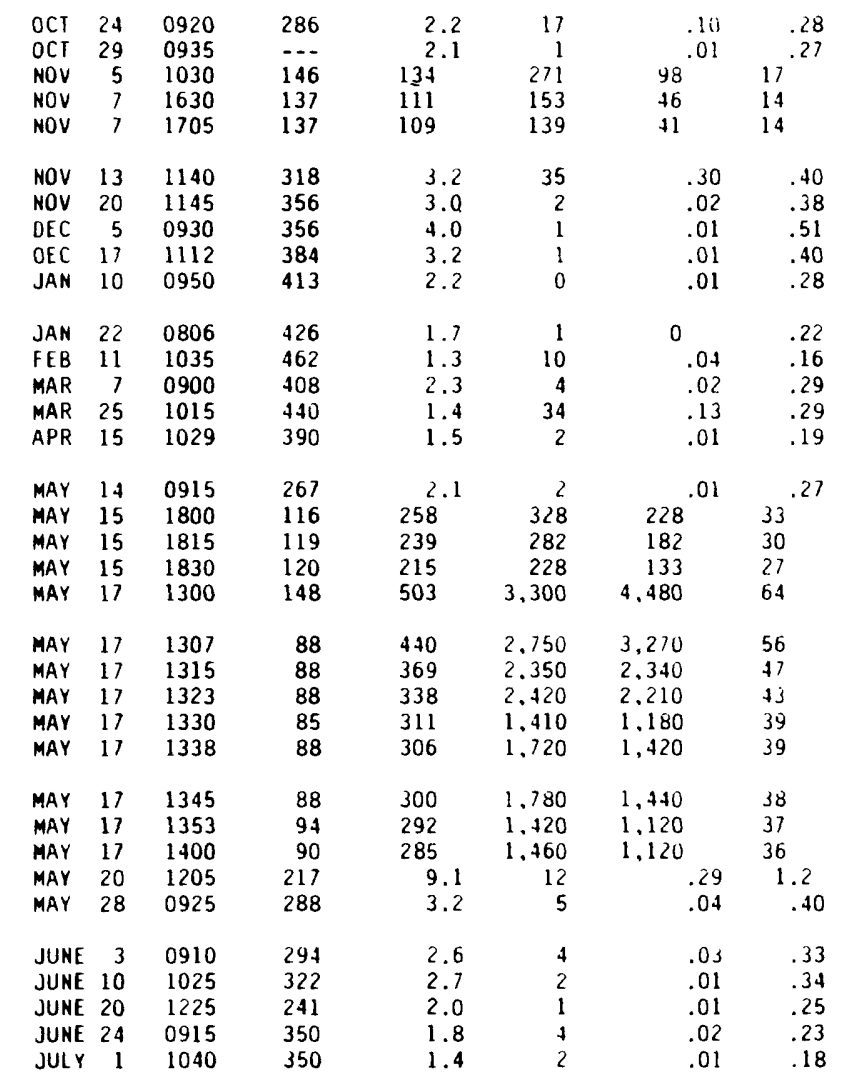

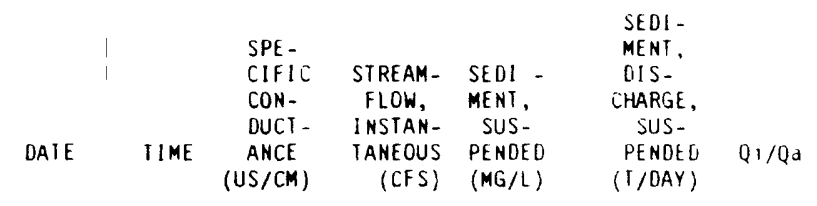

WATER YEAR OCTOBER 1984 TO SEPIEMBER 1985

\begin{tabular}{lr|rrrrll} 
JULY & 15 & 0835 & 302 & 1.7 & 4 & 0.02 & 0.22 \\
JULY & 16 & 1250 & 230 & 9.8 & 82 & 2.2 & 1.2 \\
JULY & 22 & 1220 & 306 & 1.7 & 1 & 0 & .22 \\
JULY 29 & 1030 & 313 & 2.1 & 0 & 0 & .27 \\
AUG & 5 & 1335 & 310 & 1.4 & 1 & 0 & .18 \\
& & & & & & \\
AUG & 12 & 0935 & 310 & 1.3 & 1 & 0 & .16 \\
AUG 28 & 1330 & 245 & 4.4 & 0 & 0 & .56 \\
SEPI & 3 & 0930 & 275 & 4.2 & 5 & .06 & .53 \\
SEPT & 9 & 0920 & 250 & 6.2 & 18 & .30 & .78 \\
SEPT 16 & 1125 & 235 & 7.4 & 4 & .08 & .94 \\
& & & & & & & \\
SEPT 24 & 0945 & 310 & 21 & 110 & 6.2 & 2.7 \\
SEPI & 24 & 1000 & 320 & 23 & 147 & 9.1 & 2.9 \\
& & & & & & &
\end{tabular}

\begin{tabular}{lr|rrrrrr} 
OCT & 7 & 1005 & 120 & 94 & 274 & 70 & 12 \\
OCT & 7 & 1010 & 120 & 93 & 178 & 45 & 12 \\
OCT & 7 & 1455 & 130 & 52 & 106 & 15 & 6.6 \\
OCT & 7 & 1525 & 130 & 48 & 88 & 11 & 6.1 \\
OCT & 15 & 0915 & 320 & .77 & 1 & 0 & .10 \\
& & & & & & & \\
OCT & 31 & 0905 & 160 & 56 & 121 & 18 & 7.1 \\
OCT & 31 & 0925 & 170 & 53 & 104 & 15 & 6.7 \\
NOV & 12 & 1010 & 330 & 19 & 97 & 5.0 & 2.4 \\
NOV & 18 & 0955 & 145 & 47 & 161 & 20 & 5.9 \\
OEC & 2 & 1340 & 370 & 2.9 & 2 & .02 & .37
\end{tabular}

\begin{tabular}{ll|llll} 
DEC & 9 & 0920 & 390 & 4.4 & 3
\end{tabular}

\begin{tabular}{ll|llllll}
$\mathrm{DEC}$ & 9 & 0920 & 390 & 4.4 & 3 & .04 & .56
\end{tabular}

\begin{tabular}{ll|llllll}
$D E C$ & 16 & 1010 & 370 & 3.7 & 5 & .05 & .47 \\
DEC & 23 & 0840 & 400 & 3.4 & 3 & .03 & .43
\end{tabular}

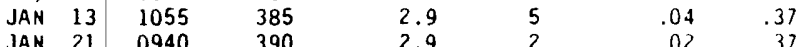

JAN $21 \quad 0940 \quad 390 \quad 270$

\begin{tabular}{lr|rrrrrr} 
JAN & 27 & 1210 & 410 & 1.9 & 2 & .01 & .24 \\
FE8 & 3 & 0905 & 470 & 3.5 & 3 & .03 & .44
\end{tabular}

\begin{tabular}{ll|llllll} 
FE8 & 24 & 0840 & 430 & 1.3 & 1 & .03 & .44 \\
MAR & 3 & 1435 & 430 & 1.2 & 1 & .01
\end{tabular}

\begin{tabular}{rr|rrrrrr} 
MAR & 3 & 1435 & 430 & 1.2 & 1 & .01 & .16 \\
MAR & 17 & 0940 & 410 & 1.6 & 1 & .01 & .15
\end{tabular}

$\begin{array}{llllllll}\text { MAR } & 31 & 0925 & 400 & 1.8 & 1 & .01 & .23\end{array}$

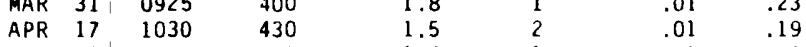

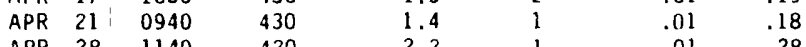

$\begin{array}{lrrrrrrr}\text { APR } & 28 & 1140 & 420 & 2.2 & 1 & .01 & .28 \\ \text { MAY } & 5 & 0940 & 420 & 1.6 & 1 & .01 & .20\end{array}$

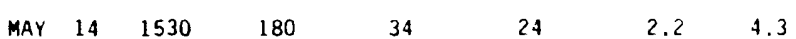

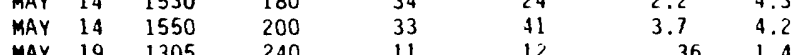

$\begin{array}{rrrrrrr}\text { MAY } & 19 & 1305 & 240 & 11 & 12 & .36 \\ \text { JUNE } 2 & 1305 & 300 & 4.1 & 6 & .07 & .52\end{array}$

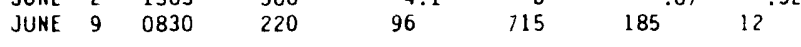

$\begin{array}{llllllll}\text { JUNE } & 9 & 0840 & 190 & 117 & 788 & 249 & 15\end{array}$

\begin{tabular}{rr|rrrrrr} 
JUNE & 9 & 0850 & 160 & 117 & 887 & 280 & 15 \\
JUNE & 9 & 0900 & 150 & 95 & 1,040 & 267 & 12
\end{tabular}

\begin{tabular}{rr|rrrrrr} 
JUNE & 9 & 0900 & 150 & 95 & 1,040 & 267 & 12 \\
JUNE & 9 & 0915 & 140 & 78 & 773 & 163 & 9.9 \\
JUNE & 9 & 0930 & 160 & 63 & 529 & 90 & 8.0
\end{tabular}

$\begin{array}{lllllll}\text { JUNE } 23 & 0925 & 380 & 1.8 & 1 & 0 & .23\end{array}$

$\begin{array}{lrllllll}\text { JUIY } & 1 & 0940 & 410 & 1.8 & 1 & 0 & .23 \\ \text { AUG } & 4 & 1000 & 410 & 1.9 & 2 & .01 & .24 \\ \end{array}$

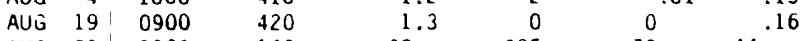

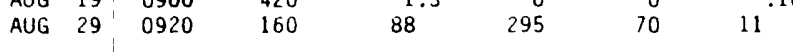

\begin{tabular}{ll|llllll} 
AUG 29 & 0935 & 170 & 81 & 265 & 58 & 10
\end{tabular}

$\begin{array}{rrrrrrrr}\text { SEPT } & 8 & 0930 & 340 & 3.5 & 0 & 0 & .44\end{array}$

$\begin{array}{llllllll}\text { SEPT } & 22 & 1320 & 380 & 1.5 & 2 & .01 & .19 \\ \text { SEPI } 29 & 0930 & 330 & 1.8 & 6 & .03 & .23\end{array}$ 


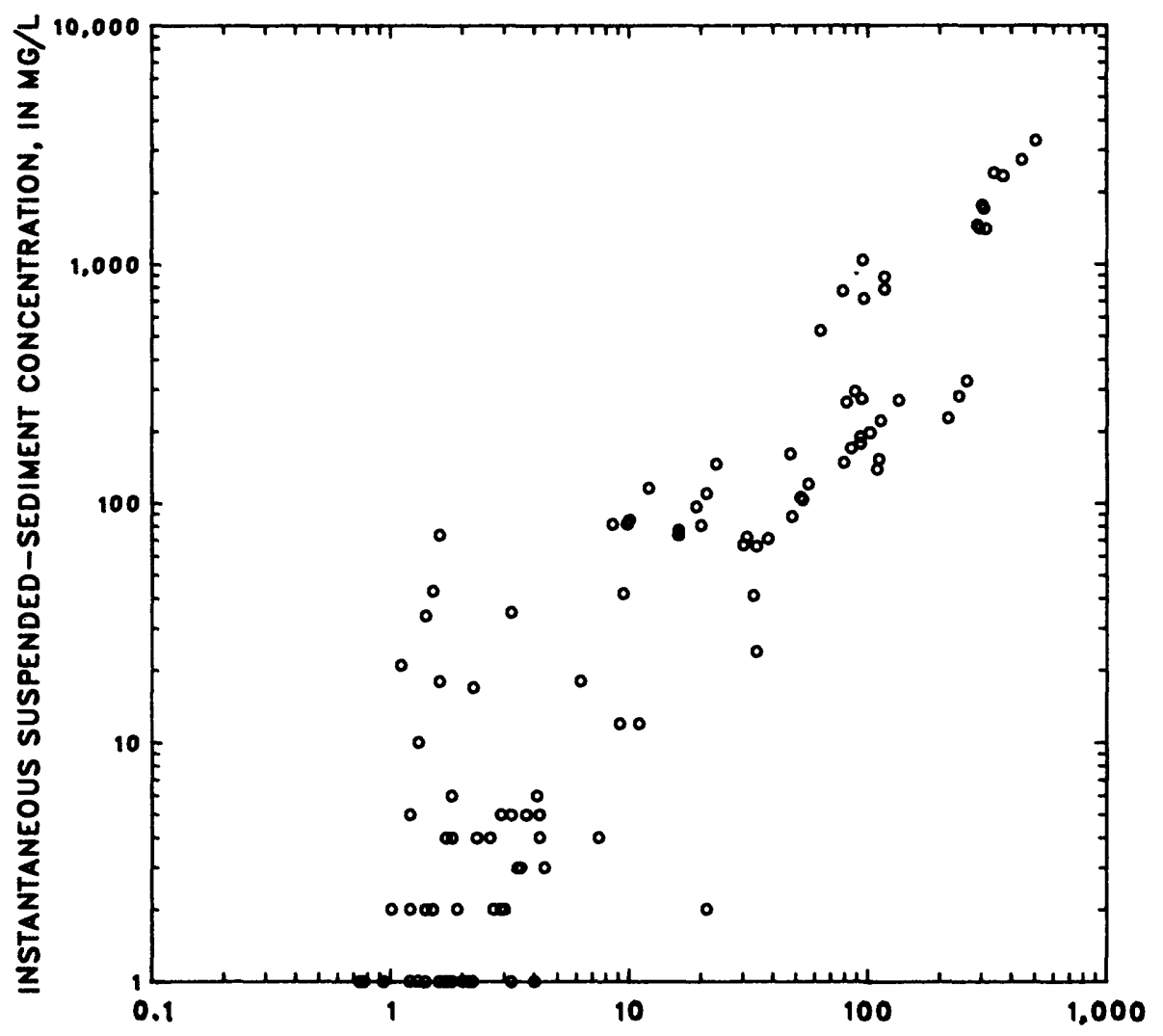

INSTANTANEOUS WATER DISCHARGE, IN CUBIC FEET PER SECOND

Figure 6.--Relation of suspended-sediment concentration to stream discharge for station Quebrada Salvatierra near San Lorenzo. 
Table 7. Suspended-sediment data for samples collected at station Rio Cayaguas at Cerro Gordo

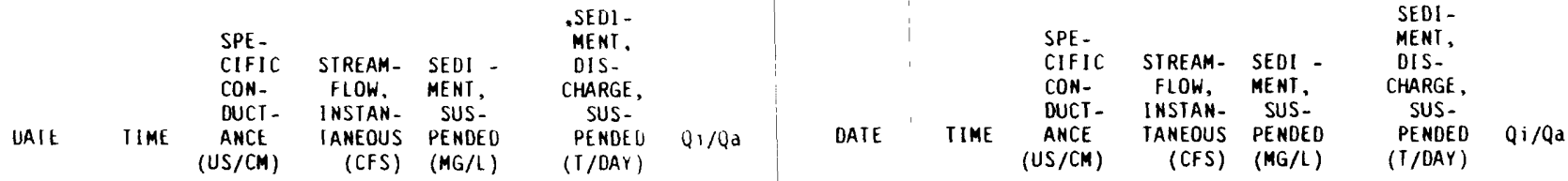

WATER YEAR DCTOBER 1983 TD SEPTEMBER 1984

\begin{tabular}{|c|c|c|c|c|c|c|c|}
\hline $\begin{array}{l}\text { FEB } \\
\text { FEB } \\
\text { FEB } \\
\text { FEB } \\
\text { FEB }\end{array}$ & $\begin{array}{l}14 \\
14 \\
14 \\
14 \\
14\end{array}$ & $\begin{array}{l}1215 \\
1235 \\
1238 \\
1241 \\
1244\end{array}$ & $\begin{array}{l}115 \\
115 \\
\ldots- \\
\ldots- \\
\ldots-\end{array}$ & $\begin{array}{l}43 \\
42 \\
42 \\
42 \\
42\end{array}$ & $\begin{array}{l}72 \\
66 \\
74 \\
73 \\
76\end{array}$ & $\begin{array}{l}8.4 \\
7.7 \\
8.4 \\
8.3 \\
8.6\end{array}$ & $\begin{array}{r}0.87 \\
.85 \\
.85 \\
.85 \\
.85\end{array}$ \\
\hline $\begin{array}{l}\text { FEB } \\
\text { FEB } \\
\text { FEB } \\
\text { FEB } \\
\text { MAR }\end{array}$ & $\begin{array}{l}14 \\
14 \\
14 \\
14 \\
12\end{array}$ & $\begin{array}{l}1247 \\
1250 \\
1253 \\
1255 \\
1345\end{array}$ & $\begin{array}{l}\cdots- \\
\cdots \\
\cdots \\
137\end{array}$ & $\begin{array}{l}42 \\
42 \\
42 \\
42 \\
24\end{array}$ & $\begin{array}{r}86 \\
97 \\
86 \\
74 \\
1\end{array}$ & $\begin{array}{l}10 \\
11 \\
10 \\
8.6 \\
.06\end{array}$ & $\begin{array}{l}.85 \\
.85 \\
.85 \\
.85 \\
.48\end{array}$ \\
\hline $\begin{array}{l}\text { MAR } \\
\text { APR } \\
\text { APR } \\
\text { MAY } \\
\text { AUG }\end{array}$ & $\begin{array}{l}27 \\
11 \\
24 \\
17 \\
27\end{array}$ & $\begin{array}{l}0845 \\
1315 \\
1115 \\
1245 \\
1405\end{array}$ & $\begin{array}{l}141 \\
140 \\
142 \\
148 \\
115\end{array}$ & $\begin{array}{l}18 \\
14 \\
16 \\
12 \\
41\end{array}$ & $\begin{array}{r}6 \\
4 \\
2 \\
4 \\
67\end{array}$ & $\begin{array}{l}.29 \\
.15 \\
.09 \\
.13 \\
7.4\end{array}$ & $\begin{array}{l}.36 \\
.28 \\
.32 \\
.24 \\
.83\end{array}$ \\
\hline $\begin{array}{l}\text { SEPT } \\
\text { SEPT } \\
\text { SEPT } \\
\text { SEPT }\end{array}$ & $\begin{array}{l}12 \\
12 \\
13 \\
18\end{array}$ & $\begin{array}{l}1540 \\
1600 \\
1120 \\
1215\end{array}$ & $\begin{array}{l}115 \\
115 \\
127 \\
100\end{array}$ & $\begin{array}{l}61 \\
61 \\
44 \\
63\end{array}$ & $\begin{array}{r}126 \\
122 \\
66 \\
158\end{array}$ & $\begin{array}{c}21 \\
20 \\
7.4 \\
27\end{array}$ & $\begin{array}{c}1.2 \\
1.2 \\
.89 \\
1.3\end{array}$ \\
\hline
\end{tabular}

WATER YEAR OCTOBER 1984 TO SIPTEMBER 1985

\begin{tabular}{|c|c|c|c|c|c|c|c|}
\hline $0 C \mathrm{~T}$ & 25 & 1118 & 112 & 72 & 243 & 47 & 1.5 \\
\hline $0 C T$ & 26 & 1030 & 102 & 93 & 279 & 61 & 1.9 \\
\hline $\mathrm{OCI}$ & 29 & 1125 & 112 & 44 & 35 & 4.2 & .89 \\
\hline $\mathrm{OCT}$ & 31 & 1000 & 123 & 41 & 29 & 3.2 & .83 \\
\hline NOV & 5 & 1410 & 35 & 1,210 & 4,200 & 13,800 & $24^{\circ}$ \\
\hline NOV & 7 & 1119 & 75 & 236 & 337 & 215 & 4.8 \\
\hline NOV & 7 & 1218 & 75 & 220 & 327 & 294 & 4.4 \\
\hline NOV & 9 & 1015 & 115 & 86 & 184 & 43 & 1.1 \\
\hline NOV & 13 & 1350 & 124 & 51 & 46 & 6.3 & 1.0 \\
\hline NOV & 14 & 1125 & 124 & 50 & 49 & 6.6 & 1.0 \\
\hline NOV & 15 & 0955 & 116 & 60 & 98 & 16 & 1.2 \\
\hline NOV & 20 & 1330 & - & 42 & 53 & 6.0 & .85 \\
\hline NOV & 23 & 0850 & $\ldots$ & 47 & 75 & 10 & .95 \\
\hline NOV & 26 & 1215 & - - & 50 & 148 & 20 & 1.0 \\
\hline NOV & 28 & 0925 & $\cdots$ & 39 & 45 & 4.7 & 0.79 \\
\hline NOV & 30 & 0930 & $\cdots$ & 39 & 52 & 5.5 & .79 \\
\hline$D E C$ & 3 & 1145 & $\cdots$ & 45 & 150 & 18 & .91 \\
\hline DEC & 5 & 1115 & $\ldots$ & 38 & 71 & 7.3 & .77 \\
\hline DEC & 10 & 1206 & $\ldots$ & 36 & 54 & 5.2 & .73 \\
\hline DEC & 12 & 1035 & 138 & 37 & 86 & 8.6 & .75 \\
\hline UEC. & 13 & 1055 & 138 & 36 & 31 & 3.0 & .73 \\
\hline DEC & 17 & 1240 & 132 & 39 & 29 & 3.0 & .79 \\
\hline DEC & 19 & 1125 & 128 & 32 & 17 & 1.5 & .65 \\
\hline$O E C$ & 20 & 1255 & 128 & 32 & 11 & .95 & .65 \\
\hline OEC & 26 & 0838 & 128 & 33 & 57 & 5.1 & .65 \\
\hline LEC C & 27 & 1004 & 137 & 33 & 33 & 2.9 & .65 \\
\hline JAN & 8 & 1050 & 137 & 28 & 10 & .76 & .57 \\
\hline JAN & 10 & 1123 & 140 & 26 & 9 & .63 & .53 \\
\hline JAN & 15 & 1100 & 149 & 25 & 7 & .47 & .51 \\
\hline JAN & 17 & 1023 & 143 & 24 & 15 & .97 & .48 \\
\hline JAN & 18 & 1000 & 140 & 25 & 12 & .81 & .51 \\
\hline JAN & 21 & 1000 & 143 & 25 & 5 & .34 & .51 \\
\hline JAN & 25 & 0800 & 140 & 24 & 10 & .65 & .48 \\
\hline JAN & 28 & 0950 & 140 & 20 & 5 & .27 & .40 \\
\hline JAN & 30 & 1100 & 140 & 19 & 6 & .30 & .38 \\
\hline
\end{tabular}

WATER YEAR OCTOBER 1984 TO SEPIEMBER 1985

\begin{tabular}{|c|c|c|c|c|c|c|c|}
\hline $\begin{array}{l}\text { FEB } \\
\text { FEB } \\
\text { FEB } \\
\text { FEB } \\
\text { FEB }\end{array}$ & $\begin{array}{r}1 \\
4 \\
6 \\
8 \\
14\end{array}$ & $\begin{array}{l}1230 \\
1100 \\
1035 \\
1110 \\
1000\end{array}$ & $\begin{array}{l}142 \\
141 \\
147 \\
145 \\
150\end{array}$ & $\begin{array}{l}22 \\
17 \\
17 \\
15 \\
15\end{array}$ & $\begin{array}{r}7 \\
7 \\
9 \\
13 \\
36\end{array}$ & $\begin{array}{r}0.38 \\
.32 \\
.41 \\
.53 \\
1.5\end{array}$ & $\begin{array}{r}0.44 \\
.34 \\
.34 \\
.30 \\
.30\end{array}$ \\
\hline $\begin{array}{l}\text { FEB } \\
\text { FEB } \\
\text { FEB } \\
\text { FEB } \\
\text { MAR }\end{array}$ & $\begin{array}{r}19 \\
20 \\
22 \\
26 \\
1\end{array}$ & $\begin{array}{l}1020 \\
0910 \\
1045 \\
1030 \\
0924\end{array}$ & $\begin{array}{l}150 \\
139 \\
134 \\
139 \\
134\end{array}$ & $\begin{array}{l}22 \\
30 \\
20 \\
19 \\
18\end{array}$ & $\begin{array}{l}22 \\
78 \\
21 \\
14 \\
30\end{array}$ & $\begin{array}{l}1.3 \\
6.3 \\
1.1 \\
.70 \\
1.5\end{array}$ & $\begin{array}{l}.44 \\
.61 \\
.40 \\
.38 \\
.36\end{array}$ \\
\hline $\begin{array}{l}\text { MAR } \\
\text { MAR } \\
\text { MAR } \\
\text { MAR } \\
\text { MAR }\end{array}$ & $\begin{array}{r}5 \\
6 \\
7 \\
11 \\
13\end{array}$ & $\begin{array}{l}1100 \\
1115 \\
1144 \\
0945 \\
0805\end{array}$ & $\begin{array}{l}139 \\
143 \\
117 \\
144 \\
140\end{array}$ & $\begin{array}{l}14 \\
15 \\
43 \\
21 \\
19\end{array}$ & $\begin{array}{r}11 \\
63 \\
151 \\
153 \\
9\end{array}$ & $\begin{array}{c}. .42 \\
2.6 \\
18 \\
8.7 \\
.46\end{array}$ & $\begin{array}{l}.28 \\
.30 \\
.87 \\
.42 \\
.38\end{array}$ \\
\hline $\begin{array}{l}\text { MAR } \\
\text { MAR } \\
\text { MAR } \\
\text { MAR } \\
\text { MAR }\end{array}$ & $\begin{array}{l}15 \\
19 \\
20 \\
21 \\
25\end{array}$ & $\begin{array}{l}0830 \\
0845 \\
0910 \\
1150 \\
1145\end{array}$ & $\begin{array}{l}145 \\
130 \\
140 \\
140 \\
145\end{array}$ & $\begin{array}{l}20 \\
23 \\
18 \\
19 \\
17\end{array}$ & $\begin{array}{r}9 \\
54 \\
11 \\
8 \\
8\end{array}$ & $\begin{array}{c}.49 \\
3.4 \\
.53 \\
.41 \\
.37\end{array}$ & $\begin{array}{r}.40 \\
.46 \\
0.36 \\
.38 \\
.34\end{array}$ \\
\hline $\begin{array}{l}\text { MAR } \\
\text { MAR } \\
\text { MAR } \\
\text { APR } \\
\text { APR }\end{array}$ & $\begin{array}{r:}27 \\
29 \\
29 \\
1 \\
8\end{array}$ & $\begin{array}{l}1006 \\
1116 \\
1125 \\
0940 \\
1026\end{array}$ & $\begin{array}{r}140 \\
65 \\
65 \\
130 \\
135\end{array}$ & $\begin{array}{r}20 \\
453 \\
397 \\
24 \\
22\end{array}$ & $\begin{array}{r}5 \\
1,450 \\
1,230 \\
33 \\
19\end{array}$ & $\begin{array}{r}1,770^{.27} \\
1,320 \\
2.1 \\
1.1\end{array}$ & $\begin{array}{l}.40 \\
9.2 \\
8.0 \\
.48 \\
.44\end{array}$ \\
\hline $\begin{array}{l}\text { APR } \\
\text { APR } \\
\text { APR } \\
\text { MAY } \\
\text { MAY }\end{array}$ & $\begin{array}{l}10 \\
12 \\
26 \\
15 \\
15\end{array}$ & $\begin{array}{l}0916 \\
1100 \\
1300 \\
1235 \\
1240\end{array}$ & $\begin{array}{r}135 \\
135 \\
110 \\
68 \\
65\end{array}$ & $\begin{array}{r}19 \\
19 \\
34 \\
548 \\
652\end{array}$ & $\begin{array}{r}9 \\
5 \\
91 \\
2.240 \\
1.840\end{array}$ & $\begin{array}{rl} & .46 \\
.26 & 8.4 \\
3,310 \\
3.240\end{array}$ & $\begin{array}{l}.38 \\
.38 \\
11^{.69} \\
13\end{array}$ \\
\hline $\begin{array}{l}\text { MAY } \\
\text { MAY } \\
\text { MAY } \\
\text { MAY } \\
\text { MAY }\end{array}$ & $\begin{array}{l}15 \\
15 \\
15 \\
15 \\
15\end{array}$ & $\begin{array}{l}1250 \\
1255 \\
1300 \\
1305 \\
1315\end{array}$ & $\begin{array}{l}64 \\
64 \\
62 \\
60 \\
54\end{array}$ & $\begin{array}{r}917 \\
1,080 \\
1,240 \\
1,390 \\
1,670\end{array}$ & $\begin{array}{l}1,820 \\
2,510 \\
3,040 \\
3,790 \\
4,450\end{array}$ & $\begin{array}{r}4,510 \\
7,310 \\
10,200 \\
14,200 \\
20,100\end{array}$ & $\begin{array}{l}18 \\
22 \\
25 \\
28 \\
34\end{array}$ \\
\hline $\begin{array}{l}\text { MAY } \\
\text { MAY } \\
\text { MAY } \\
\text { MAY } \\
\text { MAY }\end{array}$ & $\begin{array}{l}15 \\
15 \\
15 \\
15 \\
16\end{array}$ & $\begin{array}{l}1330 \\
1345 \\
1400 \\
1415 \\
1100\end{array}$ & $\begin{array}{c}52 \\
48 \\
48 \\
50 \\
74\end{array}$ & $\begin{array}{r}1,910 \\
2,580 \\
3,240 \\
3,460 \\
175\end{array}$ & $\begin{array}{r}3,780 \\
3,530 \\
5,080 \\
5,580 \\
298\end{array}$ & $\begin{array}{r}19,500 \\
24,600 \\
44,400 \\
52,100 \\
141\end{array}$ & $\begin{array}{l}39 \\
52 \\
65 \\
70 \\
3.5\end{array}$ \\
\hline $\begin{array}{l}\text { MAY } \\
\text { MAY } \\
\text { MAY } \\
\text { MAY } \\
\text { MAY }\end{array}$ & $\begin{array}{l}16 \\
17 \\
17 \\
17 \\
17\end{array}$ & $\begin{array}{l}1115 \\
1325 \\
1350 \\
1450 \\
1625\end{array}$ & $\begin{array}{l}86 \\
67 \\
72 \\
74 \\
74\end{array}$ & $\begin{array}{l}1 / 5 \\
765 \\
663 \\
495 \\
354\end{array}$ & $\begin{array}{r}432 \\
892 \\
1.820 \\
1,070 \\
732\end{array}$ & $\begin{array}{r}204 \\
1,840 \\
3,260 \\
1,430 \\
700\end{array}$ & $\begin{array}{l}3.5 \\
15 \\
13 \\
10 \\
7.2\end{array}$ \\
\hline $\begin{array}{l}\text { MAY } \\
\text { MAY } \\
\text { MAY } \\
\text { MAY } \\
\text { MAY }\end{array}$ & $\begin{array}{l}18 \\
18 \\
18 \\
18 \\
18\end{array}$ & $\begin{array}{l}1100 \\
1106 \\
1115 \\
1145 \\
1200\end{array}$ & $\begin{array}{l}36 \\
30 \\
30 \\
32 \\
30\end{array}$ & $\begin{array}{l}2,100 \\
2,040 \\
1,920 \\
1,510 \\
1,380\end{array}$ & $\begin{array}{l}4,530 \\
3,220 \\
3,350 \\
3,030 \\
3,870\end{array}$ & $\begin{array}{l}25,700 \\
17,700 \\
17,400 \\
12,400 \\
14,400\end{array}$ & $\begin{array}{l}42 \\
41 \\
39 \\
30 \\
28\end{array}$ \\
\hline $\begin{array}{l}\text { MAY } \\
\text { MAY } \\
\text { MAY } \\
\text { MAY } \\
\text { MAY }\end{array}$ & $\begin{array}{l}18 \\
18 \\
18 \\
18 \\
20\end{array}$ & $\begin{array}{l}1215 \\
1230 \\
1300 \\
1330 \\
1530\end{array}$ & $\begin{array}{l}32 \\
38 \\
35 \\
37 \\
96\end{array}$ & $\begin{array}{r}1,350 \\
1,190 \\
1.050 \\
861 \\
71\end{array}$ & $\begin{array}{r}2,880 \\
2,860 \\
3.040 \\
3,350 \\
680\end{array}$ & $\begin{array}{r}10,500 \\
9,190 \\
8.620 \\
7.790 \\
130\end{array}$ & $\begin{array}{l}27 \\
24 \\
21 \\
17 \\
1.4\end{array}$ \\
\hline
\end{tabular}


Table 7. Suspended-sediment data for samples collected at station Rio Cayaguas at Cerro Gordo

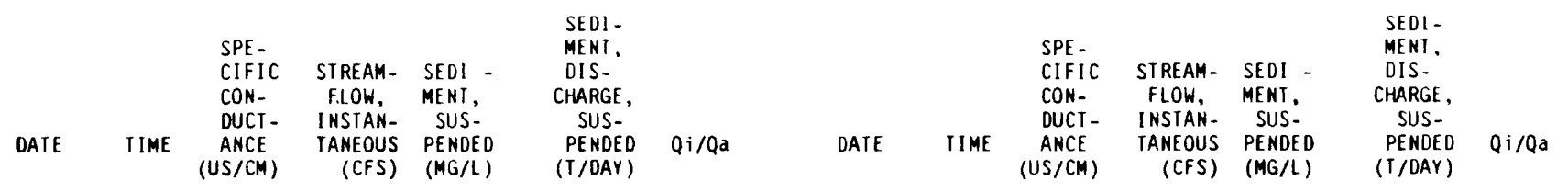

WAIER YEAR OCTOBER 1984 TO SEPIEMBER 1985-Cont inued

WATER YEAR OCTOBER 1984 TO SEPTEMBER 1985

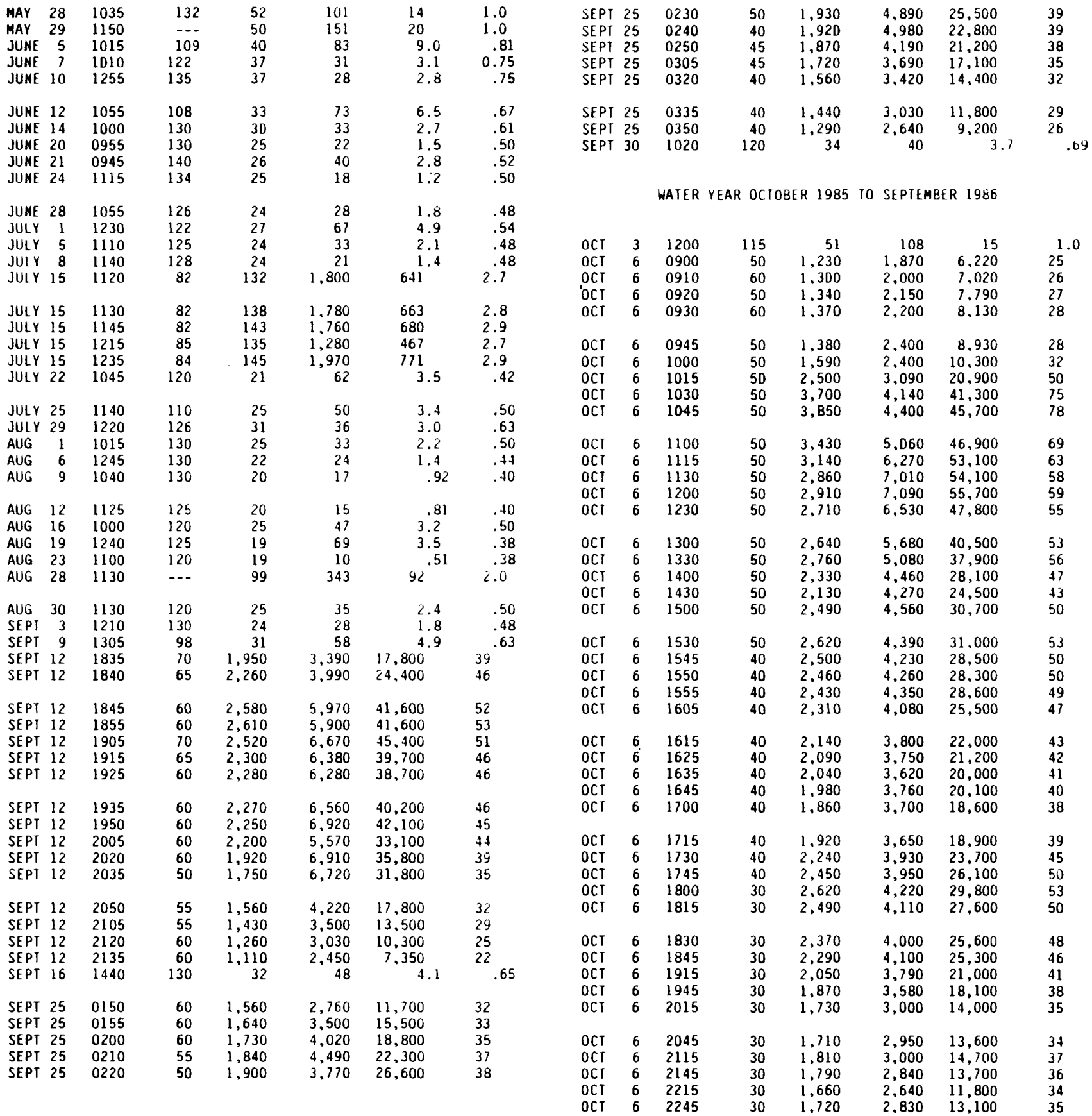




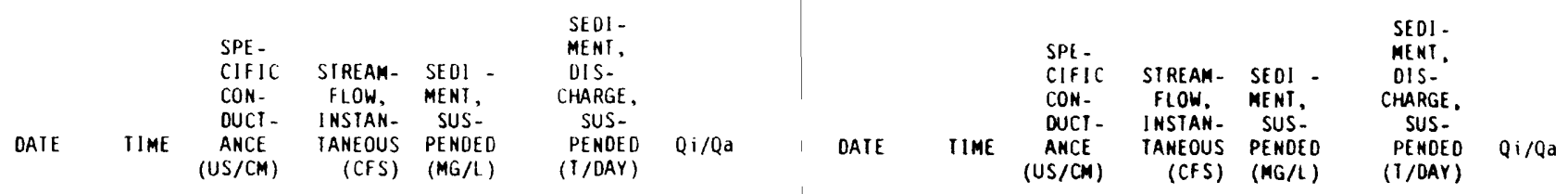

WATER YEAR OCTOBER 1985 IO SEPIEMBER 1986 -Cont inued

\begin{tabular}{|c|c|c|c|c|c|c|c|c|c|c|c|c|c|c|c|}
\hline $\begin{array}{l}O C \mathrm{OI} \\
0 \mathrm{CI} \\
O C \mathrm{~T} \\
\mathrm{OCI} \\
0 \mathrm{CI}\end{array}$ & $\begin{array}{l}15 \\
18 \\
24 \\
24 \\
24\end{array}$ & $\begin{array}{l}1115 \\
1040 \\
0900 \\
0910 \\
0920\end{array}$ & $\begin{array}{r}120 \\
110 \\
60 \\
60 \\
60\end{array}$ & $\begin{array}{r}27 \\
49 \\
1,490 \\
1,610 \\
1,690\end{array}$ & $\begin{array}{r}33 \\
200 \\
5,880 \\
3,040 \\
3,130\end{array}$ & $\begin{array}{r}2.4 \\
26 \\
23,700 \\
13,200 \\
14,300\end{array}$ & $\begin{array}{l}0.54 \\
30 \\
32 \\
34 \\
34\end{array}$ & $\begin{array}{l}\text { FEB } \\
\text { MAR } \\
\text { MAR } \\
\text { MAR } \\
\text { MAR }\end{array}$ & $\begin{array}{r}24 \\
3 \\
14 \\
20 \\
24\end{array}$ & $\begin{array}{l}1015 \\
1345 \\
1125 \\
0905 \\
1015\end{array}$ & $\begin{array}{l}140 \\
140 \\
150 \\
145 \\
160\end{array}$ & $\begin{array}{l}16 \\
17 \\
25 \\
16 \\
17\end{array}$ & $\begin{array}{r}13 \\
12 \\
3 \\
2 \\
6\end{array}$ & $\begin{array}{l}.56 \\
.55 \\
.20 \\
.09 \\
.28\end{array}$ & $\begin{array}{l}.32 \\
.34 \\
.50 \\
.32 \\
.34\end{array}$ \\
\hline $\begin{array}{l}O C I \\
O C I \\
O C I \\
O C I \\
0 C 1\end{array}$ & $\begin{array}{l}24 \\
24 \\
24 \\
24 \\
29\end{array}$ & $\begin{array}{l}0930 \\
0945 \\
1000 \\
1015 \\
1230\end{array}$ & $\begin{array}{l}60 \\
60 \\
60 \\
60 \\
60\end{array}$ & $\begin{array}{r}1,730 \\
1,760 \\
1,600 \\
1,490 \\
313\end{array}$ & $\begin{array}{l}3,070 \\
3,180 \\
3,000 \\
3,090 \\
1,140\end{array}$ & $\begin{array}{r}14.300 \\
15,100 \\
13.000 \\
12.400 \\
963\end{array}$ & $\begin{array}{c}35 \\
36 \\
32 \\
30 \\
6.3\end{array}$ & $\begin{array}{l}\text { MAR } \\
\text { APR } \\
\text { APR } \\
\text { APR } \\
\text { APR }\end{array}$ & $\begin{array}{l}31 \\
10 \\
14 \\
17 \\
21\end{array}$ & $\begin{array}{l}1105 \\
1025 \\
1105 \\
0955 \\
1100\end{array}$ & $\begin{array}{l}130 \\
140 \\
150 \\
150 \\
150\end{array}$ & $\begin{array}{l}28 \\
23 \\
15 \\
16 \\
16\end{array}$ & $\begin{array}{r}145 \\
25 \\
12 \\
9 \\
6\end{array}$ & $\begin{array}{r}11 \\
1.6 \\
.49 \\
.39 \\
.26\end{array}$ & $\begin{array}{l}.57 \\
.46 \\
.30 \\
.32 \\
.32\end{array}$ \\
\hline $\begin{array}{l}\text { OCT } \\
\text { NOV } \\
\text { NOV } \\
\text { NOV } \\
\text { NOV }\end{array}$ & $\begin{array}{l}31 \\
18 \\
12 \\
26 \\
27\end{array}$ & $\begin{array}{l}1110 \\
1135 \\
1140 \\
1020 \\
1140\end{array}$ & $\begin{array}{r}80 \\
90 \\
115 \\
120 \\
120\end{array}$ & $\begin{array}{r}156 \\
134 \\
72 \\
42 \\
47\end{array}$ & $\begin{array}{r}479 \\
254 \\
105 \\
36 \\
29\end{array}$ & $\begin{array}{r}202 \\
92 \\
20 \\
4.1 \\
3.1\end{array}$ & $\begin{array}{l}3.2 \\
2.7 \\
1.5 \\
.85 \\
.95\end{array}$ & $\begin{array}{l}\text { MAY } \\
\text { MAY } \\
\text { MAY } \\
\text { MAY } \\
\text { JUNE }\end{array}$ & $\begin{array}{r}5 \\
14 \\
14 \\
22 \\
2\end{array}$ & $\begin{array}{l}1055 \\
1445 \\
1500 \\
1030 \\
1150\end{array}$ & $\begin{array}{r}135 \\
90 \\
100 \\
140 \\
140\end{array}$ & $\begin{array}{r}24 \\
134 \\
140 \\
19 \\
28\end{array}$ & $\begin{array}{r}47 \\
272 \\
260 \\
74 \\
111\end{array}$ & $\begin{array}{l}3.0 \\
98 \\
98 \\
3.8 \\
8.4\end{array}$ & $\begin{array}{l}.48 \\
2.7 \\
2.8 \\
.38 \\
.57\end{array}$ \\
\hline $\begin{array}{l}\text { DEC } \\
D E C \\
D E C \\
D E C \\
D E C\end{array}$ & $\begin{array}{r}6 \\
9 \\
12 \\
16 \\
23\end{array}$ & $\begin{array}{l}1125 \\
1120 \\
0945 \\
1215 \\
1015\end{array}$ & $\begin{array}{l}130 \\
120 \\
120 \\
120 \\
130\end{array}$ & $\begin{array}{l}42 \\
36 \\
73 \\
40 \\
55\end{array}$ & $\begin{array}{r}76 \\
29 \\
239 \\
30 \\
72\end{array}$ & $\begin{array}{l}8.6 \\
2.8 \\
47 \\
3.2 \\
11\end{array}$ & $\begin{array}{l}.85 \\
.73 \\
1.5 \\
.81 \\
1.1\end{array}$ & $\begin{array}{l}\text { JUNE } \\
\text { JUIY } \\
\text { JULY } \\
\text { JULY } \\
\text { JULY }\end{array}$ & $\begin{array}{r}23 \\
1 \\
7 \\
14 \\
29\end{array}$ & $\begin{array}{l}1120 \\
1120 \\
1115 \\
1200 \\
1055\end{array}$ & $\begin{array}{l}150 \\
150 \\
140 \\
155 \\
160\end{array}$ & $\begin{array}{l}24 \\
34 \\
34 \\
28 \\
21\end{array}$ & $\begin{array}{l}37 \\
24 \\
45 \\
44 \\
25\end{array}$ & $\begin{array}{l}2.4 \\
2.2 \\
4.1 \\
3.3 \\
1.4\end{array}$ & $\begin{array}{l}.48 \\
.69 \\
.69 \\
.57 \\
.42\end{array}$ \\
\hline $\begin{array}{l}\text { DEC } \\
\text { JAN } \\
\text { JAN } \\
\text { JAN } \\
\text { JAN }\end{array}$ & $\begin{array}{r}27 \\
8 \\
13 \\
16 \\
21\end{array}$ & $\begin{array}{l}1020 \\
1045 \\
1240 \\
1055 \\
1140\end{array}$ & $\begin{array}{l}130 \\
130 \\
130 \\
130 \\
130\end{array}$ & $\begin{array}{l}41 \\
34 \\
35 \\
38 \\
62\end{array}$ & $\begin{array}{l}32 \\
26 \\
29 \\
23 \\
38\end{array}$ & $\begin{array}{l}3.5 \\
2.4 \\
2.7 \\
2.4 \\
6.4\end{array}$ & $\begin{array}{l}.83 \\
.69 \\
.71 \\
.77 \\
1.2\end{array}$ & $\begin{array}{l}\text { AUG } \\
\text { AUG } \\
\text { AUG } \\
\text { AUG } \\
\text { SEPT }\end{array}$ & $\begin{array}{r}1 \\
19 \\
26 \\
29 \\
8\end{array}$ & $\begin{array}{l}1155 \\
1030 \\
1120 \\
1130 \\
1125\end{array}$ & $\begin{array}{r}150 \\
160 \\
170 \\
75 \\
140\end{array}$ & $\begin{array}{r}20 \\
14 \\
13 \\
381 \\
16\end{array}$ & $\begin{array}{r}24 \\
27 \\
17 \\
455 \\
12\end{array}$ & $\begin{array}{r}1.3 \\
1.0 \\
468 \\
.60 \\
.52\end{array}$ & $\begin{array}{l}.40 \\
.28 \\
.26 \\
7.7 \\
.32\end{array}$ \\
\hline $\begin{array}{l}\text { JAN } \\
\text { JAN } \\
\text { FEB } \\
\text { FEB } \\
\text { FEB }\end{array}$ & $\begin{array}{r}27 \\
30 \\
3 \\
13 \\
18\end{array}$ & $\begin{array}{l}1050 \\
1100 \\
1050 \\
1200 \\
1135\end{array}$ & $\begin{array}{l}130 \\
130 \\
130 \\
145 \\
140\end{array}$ & $\begin{array}{l}14 \\
21 \\
17 \\
17 \\
24\end{array}$ & $\begin{array}{l}14 \\
30 \\
12 \\
13 \\
17\end{array}$ & $\begin{array}{r}.53 \\
1.7 \\
.55 \\
.60 \\
1.1\end{array}$ & $\begin{array}{l}.28 \\
.42 \\
.34 \\
.34 \\
.48\end{array}$ & $\begin{array}{l}\text { SEPT } \\
\text { SEPT } \\
\text { SEPI } \\
\text { SEPT }\end{array}$ & $\begin{array}{l}15 \\
19 \\
22 \\
29\end{array}$ & $\begin{array}{l}1245 \\
1010 \\
1200 \\
1135\end{array}$ & $\begin{array}{l}140 \\
140 \\
140 \\
130\end{array}$ & $\begin{array}{l}16 \\
10 \\
25 \\
14\end{array}$ & $\begin{array}{l}17 \\
18 \\
16 \\
15\end{array}$ & $\begin{array}{l}.73 \\
.49 \\
1.1 \\
.57\end{array}$ & $\begin{array}{l}.32 \\
.20 \\
.50 \\
.28\end{array}$ \\
\hline
\end{tabular}

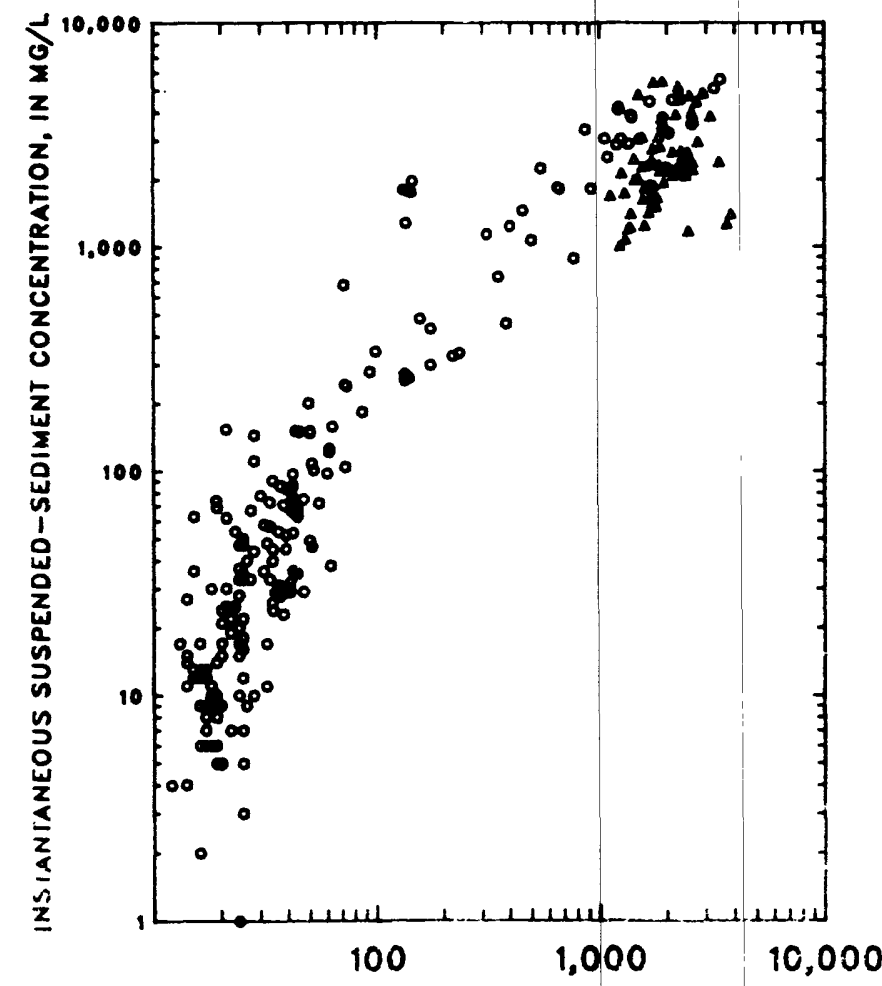

EXPLANATION

- FIELD SAMPLE

- isco SAMPLE 
Table 8. Suspended-sediment data for samples collected at station Rio Turabo at Borinquen

\begin{tabular}{|c|c|c|c|c|c|c|c|c|c|c|c|c|}
\hline DATE & TIME & $\begin{array}{l}\text { SPE- } \\
\text { CIFIC } \\
\text { CON- } \\
\text { DUCT- } \\
\text { ANCE } \\
\text { (US/CM) }\end{array}$ & $\begin{array}{l}\text { SIREAM- } \\
\text { FLOW, } \\
\text { INSTAN- } \\
\text { TANEOUS } \\
\text { (CFS) }\end{array}$ & $\begin{array}{l}\text { SEDI - } \\
\text { MENT. } \\
\text { SUS- } \\
\text { PENDED } \\
(M G / L)\end{array}$ & $\begin{array}{l}\text { SEDI - } \\
\text { MENT. } \\
\text { DIS- } \\
\text { CHARGE, } \\
\text { SUS- } \\
\text { PENDED } \\
\text { (T/DAY) }\end{array}$ & $Q i / Q \mathbf{a}$ & DATE & TIME & $\begin{array}{l}\text { SPE- } \\
\text { CIFIC } \\
\text { CON- } \\
\text { DUCI- } \\
\text { ANCE } \\
\text { (US/CM) }\end{array}$ & $\begin{array}{l}\text { STREAM- } \\
\text { FLOW, } \\
\text { INSTAK- } \\
\text { TANEOUS } \\
\text { (CFS) }\end{array}$ & $\begin{array}{l}\text { SEDI - } \\
\text { MENT, } \\
\text { SUS- } \\
\text { PENDED } \\
\text { (MG/L) }\end{array}$ & $\begin{array}{l}\text { SEDI - } \\
\text { MENT, } \\
\text { DIS- } \\
\text { CHARGE, } \\
\text { SUS- } \\
\text { PENDED } \\
\text { (T/DAY) }\end{array}$ \\
\hline
\end{tabular}

WATER YEAR OCTOBER 1983 TO SEPTEMBER 1984

\begin{tabular}{|c|c|c|c|c|c|c|c|}
\hline $\begin{array}{l}\text { FEB } \\
\text { MAR } \\
\text { MAR } \\
\text { APR } \\
\text { MAY }\end{array}$ & $\begin{array}{r}16 \\
9 \\
28 \\
12 \\
21\end{array}$ & $\begin{array}{l}1125 \\
1030 \\
0815 \\
1015 \\
1230\end{array}$ & $\begin{array}{l}125 \\
172 \\
184 \\
177 \\
184\end{array}$ & $\begin{array}{r}133 \\
14 \\
17 \\
9.0 \\
9.0\end{array}$ & $\begin{array}{r}63 \\
0 \\
3 \\
3 \\
12\end{array}$ & $\begin{array}{l}23 \\
0 \\
.14 \\
.07 \\
.29\end{array}$ & $\begin{array}{l}4.5 \\
.51 \\
.62 \\
.33 \\
.33\end{array}$ \\
\hline $\begin{array}{l}\text { MAY } \\
\text { MAY } \\
\text { MAY } \\
\text { MAY } \\
\text { JUNE }\end{array}$ & $\begin{array}{l}25 \\
30 \\
30 \\
30 \\
12\end{array}$ & $\begin{array}{l}1330 \\
1620 \\
1622 \\
1625 \\
1415\end{array}$ & $\begin{array}{l}\ldots . \\
\cdots- \\
103 \\
103 \\
150\end{array}$ & $\begin{array}{l}39 \\
84 \\
83 \\
91 \\
24\end{array}$ & $\begin{array}{r}20 \\
1,040 \\
1,220 \\
1,090 \\
31\end{array}$ & $\begin{array}{r}2.1 \\
236 \\
273 \\
267 \\
2.0\end{array}$ & $\begin{array}{l}1.4 \\
3.1 \\
3.0 \\
3.3 \\
.88\end{array}$ \\
\hline $\begin{array}{l}\text { JUNE } \\
\text { JULY } \\
\text { JULY } \\
\text { JULY } \\
\text { JULY }\end{array}$ & $\begin{array}{r}28 \\
5 \\
5 \\
5 \\
5\end{array}$ & $\begin{array}{l}0945 \\
1220 \\
1225 \\
1325 \\
1327\end{array}$ & $\begin{array}{l}179 \\
113 \\
119 \\
122 \\
117\end{array}$ & $\begin{array}{r}10 \\
151 \\
151 \\
127 \\
127\end{array}$ & $\begin{array}{r}0 \\
156 \\
159 \\
125 \\
129\end{array}$ & $\begin{array}{r}0 \\
64 \\
65 \\
44 \\
44\end{array}$ & $\begin{array}{l}.37 \\
5.5 \\
5.5 \\
4.6 \\
4.6\end{array}$ \\
\hline $\begin{array}{l}\text { JULY } \\
\text { JULY } \\
\text { AUG } \\
\text { SEPT } \\
\text { SEPT }\end{array}$ & $\begin{array}{r}5 \\
11 \\
23 \\
14 \\
14\end{array}$ & $\begin{array}{l}1330 \\
1545 \\
1515 \\
1430 \\
1445\end{array}$ & $\begin{array}{l}118 \\
156 \\
-\ldots \\
120 \\
121\end{array}$ & $\begin{array}{c}126 \\
23 \\
7.4 \\
36 \\
35\end{array}$ & $\begin{array}{r}137 \\
1 \\
4 \\
283 \\
287\end{array}$ & $\begin{array}{l}47 \\
.06 \\
28 \\
27\end{array}$ & $\begin{array}{l}4.6 \\
.84 \\
.27 \\
1.3 \\
1.3\end{array}$ \\
\hline $\begin{array}{l}\text { SEPT } \\
\text { SEPT } \\
\text { SEPT } \\
\text { SEPT } \\
\text { SEPT }\end{array}$ & $\begin{array}{l}14 \\
19 \\
20 \\
20 \\
20\end{array}$ & $\begin{array}{l}1500 \\
0915 \\
1005 \\
1010 \\
1015\end{array}$ & $\begin{array}{r}122 \\
108 \\
89 \\
89 \\
85\end{array}$ & $\begin{array}{r}33 \\
90 \\
183 \\
176 \\
171\end{array}$ & $\begin{array}{l}281 \\
308 \\
536 \\
636 \\
558\end{array}$ & $\begin{array}{r}25 \\
75 \\
265 \\
302 \\
258\end{array}$ & $\begin{array}{l}1.2 \\
3.3 \\
6.7 \\
6.4 \\
6.3\end{array}$ \\
\hline $\begin{array}{l}\text { SEPT } \\
\text { SEPI } \\
\text { SEPI } \\
\text { SEPT } \\
\text { SEPI } \\
\text { SEPI }\end{array}$ & $\begin{array}{l}20 \\
20 \\
20 \\
20 \\
20 \\
20\end{array}$ & $\begin{array}{l}1020 \\
1025 \\
1030 \\
1035 \\
1040 \\
1045\end{array}$ & $\begin{array}{l}85 \\
89 \\
89 \\
90 \\
90 \\
90\end{array}$ & $\begin{array}{l}165 \\
160 \\
155 \\
151 \\
148 \\
144\end{array}$ & $\begin{array}{l}543 \\
512 \\
500 \\
445 \\
621 \\
619\end{array}$ & $\begin{array}{l}242 \\
221 \\
209 \\
181 \\
248 \\
241\end{array}$ & $\begin{array}{l}6.0 \\
5.9 \\
5.7 \\
5.5 \\
5.4 \\
5.3\end{array}$ \\
\hline
\end{tabular}

\section{WATER YEAR OCTOBER 1984 TO SEPTEMBER 1985}

$\begin{array}{lrllcccc}\text { OCT } & 22 & 1313 & 149 & 17 & 33 & 1.5 & 0.62 \\ \text { OCT } & 29 & 0900 & -2 . & 14 & 15 & .56 & .51 \\ \text { NOV } & 13 & 1040 & 152 & 46 & 18 & 2.2 & 1.7 \\ \text { MOV } & 20 & 1050 & 155 & 20 & 5 & .28 & .73 \\ \text { DEC } & 5 & 0847 & 160 & 19 & 3 & .15 & .70 \\ \text { DEC } & 17 & 1025 & 159 & 15 & 9 & .36 & .55 \\ \text { JAN } & 11 & 0945 & 183 & 11 & 8 & .24 & .40 \\ \text { JAN } & 28 & 0835 & 178 & 8.8 & 4 & .09 & .32 \\ & & & & & & & .31 \\ \text { FEB } & 11 & 0930 & 182 & 8.8 & 13 & .32 \\ \text { MAR } & 6 & 1357 & 182 & 99 & 870 & 232 & 3.6 \\ \text { MAR } & 6 & 1410 & 163 & 92 & 591 & 147 & 3.4 \\ \text { MAR } & 6 & 1415 & 168 & 92 & 527 & 131 & 3.4 \\ \text { MAR } & 6 & 1430 & 160 & 77 & 463 & 96 & 2.8 \\ & & & & & & & \\ \text { MAR } & 6 & 1445 & 152 & 68 & 398 & 73 & 2.5 \\ \text { MAR } & 11 & 0815 & 167 & 16 & 5 & .22 & .59 \\ \text { MAR } & 25 & 0405 & 175 & 11 & 6 & .18 & .40 \\ \text { APR } & 15 & 0955 & 160 & 8.2 & 1 & .02 & .30 \\ \text { MAY } & 15 & 0830 & 117 & 123 & 65 & 22 & 4.5 \\ \text { MAY } & 15 & 0840 & 117 & 261 & 358 & 252 & 9.6 \\ \text { MAY } & 15 & 0845 & 122 & 261 & 288 & 203 & 9.6 \\ \text { MAY } & 15 & 0850 & 115 & 261 & 398 & 280 & 9.6 \\ \text { MAY } & 15 & 0900 & 113 & 223 & 504 & 303 & 8.2 \\ \text { MAY } & 15 & 0915 & 107 & 241 & 768 & 500 & 8.8 \\ & & & & & & & \end{array}$

WATER YEAR OCTOBER 1984 TO SEPTEMBER 1985

\begin{tabular}{|c|c|c|c|c|c|c|c|}
\hline $\begin{array}{l}\text { MAY } \\
\text { MAY } \\
\text { MAY } \\
\text { MAY }\end{array}$ & $\begin{array}{l}15 \\
15 \\
15 \\
15 \\
15\end{array}$ & $\begin{array}{l}0930 \\
0945 \\
1000 \\
1015 \\
1030\end{array}$ & $\begin{array}{l}97 \\
92 \\
89 \\
92 \\
8 B\end{array}$ & $\begin{array}{l}231 \\
223 \\
223 \\
221 \\
233\end{array}$ & $\begin{array}{l}776 \\
800 \\
584 \\
632 \\
458\end{array}$ & $\begin{array}{l}484 \\
482 \\
352 \\
377 \\
288\end{array}$ & $\begin{array}{l}8.5 \\
8.2 \\
8.2 \\
8.1 \\
B .5\end{array}$ \\
\hline $\begin{array}{l}\text { MAY } \\
\text { MAY } \\
\text { MAY } \\
\text { MAY } \\
\text { MAY }\end{array}$ & $\begin{array}{l}15 \\
15 \\
15 \\
15 \\
17\end{array}$ & $\begin{array}{l}1100 \\
1130 \\
1755 \\
1800 \\
1023\end{array}$ & $\begin{array}{l}88 \\
92 . \\
91 \\
90 \\
\text { B5 }\end{array}$ & $\begin{array}{r}202 \\
198 \\
270 \\
264 \\
3,370 .\end{array}$ & $\begin{array}{r}385 \\
340 \\
276 \\
248 \\
4,700\end{array}$ & $\begin{array}{r}210 \\
182 \\
201 \\
177 \\
42,800\end{array}$ & $\begin{array}{r}9.4 \\
7.2 \\
9.9 \\
9.7 \\
123\end{array}$ \\
\hline $\begin{array}{l}\text { MAY } \\
\text { MAY } \\
\text { MAY } \\
\text { MAY } \\
\text { MAY }\end{array}$ & $\begin{array}{l}17 \\
17 \\
17 \\
17 \\
17\end{array}$ & $\begin{array}{l}1126 \\
1130 \\
1137 \\
1145 \\
1157\end{array}$ & $\begin{array}{l}53 \\
45 \\
43 \\
43 \\
47\end{array}$ & $\begin{array}{l}6,110 \\
6,110 \\
6,120 \\
6,130 \\
6,130\end{array}$ & $\begin{array}{l}26,000 \\
28,600 \\
26,900 \\
20,300 \\
13,800\end{array}$ & $\begin{array}{l}429,000 \\
472,000 \\
445,000 \\
336,000 \\
228,000\end{array}$ & $\begin{array}{l}224 \\
224 \\
224 \\
224 \\
224\end{array}$ \\
\hline $\begin{array}{l}\text { MAY } \\
\text { MAY } \\
\text { MAY } \\
\text { MAY } \\
\text { MAY }\end{array}$ & $\begin{array}{l}17 \\
17 \\
17 \\
17 \\
17\end{array}$ & $\begin{array}{l}1200 \\
1720 \\
1725 \\
1735 \\
1750\end{array}$ & $\begin{array}{r}47 \\
93 \\
95 \\
96 \\
102\end{array}$ & $\begin{array}{l}6,130 \\
6,990 \\
6,990 \\
7,000 \\
7,100\end{array}$ & $\begin{array}{r}11,800 \\
489 \\
314 \\
440 \\
478\end{array}$ & $\begin{array}{r}195,000 \\
9,230 \\
5,930 \\
8,320 \\
9,200\end{array}$ & $\begin{array}{l}224 \\
256 \\
256 \\
256 \\
260\end{array}$ \\
\hline $\begin{array}{l}\text { MAY } \\
\text { MAY } \\
\text { JUNE } \\
\text { JUNE } \\
\text { JUHE }\end{array}$ & $\begin{array}{r}20 \\
28 \\
3 \\
10 \\
24\end{array}$ & $\begin{array}{l}1100 \\
0810 \\
0815 \\
0930 \\
0820\end{array}$ & $\begin{array}{l}124 \\
127 \\
120 \\
157 \\
150\end{array}$ & $\begin{array}{r}23 \\
11 \\
10 \\
12\end{array}$ & $\begin{array}{r}12 \\
26 \\
6 \\
4 \\
5\end{array}$ & $\begin{array}{l}1.6 \\
.18 \\
.11 \\
.16\end{array}$ & $\begin{array}{l}.84 \\
.40 \\
.37 \\
.44\end{array}$ \\
\hline $\begin{array}{l}\text { JULY } \\
\text { JULY } \\
\text { JULY } \\
\text { AUG } \\
\text { AUG }\end{array}$ & $\begin{array}{r}1 \\
8 \\
29 \\
5 \\
12\end{array}$ & $\begin{array}{l}0935 \\
0845 \\
0940 \\
0955 \\
0845\end{array}$ & $\begin{array}{l}135 \\
136 \\
148 \\
146 \\
150\end{array}$ & $\begin{array}{c}12 \\
9.5 \\
13 \\
12 \\
9.1\end{array}$ & $\begin{array}{l}4 \\
6 \\
B \\
8 \\
8\end{array}$ & $\begin{array}{l}.13 \\
.14 \\
.28 \\
.26 \\
.20\end{array}$ & $\begin{array}{l}.44 \\
.35 \\
.48 \\
.44 \\
.33\end{array}$ \\
\hline $\begin{array}{l}\text { AUG } \\
\text { SEPI } \\
\text { SEPI } \\
\text { SEPI }\end{array}$ & $\begin{array}{r}18 \\
3 \\
16 \\
24\end{array}$ & $\begin{array}{l}1025 \\
0845 \\
1010 \\
1840\end{array}$ & $\begin{array}{l}150 \\
158 \\
132 \\
140\end{array}$ & $\begin{array}{l}13 \\
13 \\
14 \\
13\end{array}$ & $\begin{array}{r}9 \\
16 \\
13 \\
75\end{array}$ & $\begin{array}{l}.31 \\
.56 \\
.49 \\
2.6\end{array}$ & $\begin{array}{l}.48 \\
.48 \\
.51 \\
.48\end{array}$ \\
\hline
\end{tabular}

WATER YEAR OCTO8ER 1985 TO SEPTEMBER 1986

$\begin{array}{lrrrrrrr}\text { OCT } & 6 & 1725 & 50 & 2,520 & 4,150 & 28,200 & 92 \\ \text { OCT } & 6 & 1730 & 50 & 2,640 & 4,870 & 34,700 & 97 \\ \text { OCT } & 6 & 1735 & 50 & 2,510 & 4,840 & 32,800 & 92 \\ \text { OCT } & 6 & 1740 & 50 & 2,380 & 4,520 & 29,000 & 87 \\ \text { OCT } & 6 & 1745 & 50 & 2,250 & 4,880 & 29,600 & 82 \\ & & & & & & & \\ \text { OCT } & 6 & 1750 & 50 & 2,120 & 4,160 & 23,800 & 18 \\ \text { OCT } & 6 & 1755 & 50 & 1,990 & 4,560 & 24,500 & 73 \\ \text { OCT } & 6 & 1800 & 50 & 1,860 & 5,340 & 26,800 & 68 \\ \text { OCI } & 6 & 1815 & 50 & 1,830 & 3,700 & 18,300 & 67 \\ \text { OCT } & 7 & 1715 & 100 & 379 & 950 & 972 & 14 \\ & & & & & & & \\ \text { OCT } & 21 & 0855 & 140 & 52 & 1 & .14 & 1.9 \\ \text { NOV } & 12 & 0850 & 140 & 28 & 3 & .19 & 1.0 \\ \text { NOV } & 18 & 0845 & 110 & 75 & 173 & 35 & 2.7 \\ \text { NOV } & 18 & 0915 & 110 & 75 & 351 & 71 & 2.7 \\ \text { DEC } & 2 & 1410 & 210 & 20 & 4 & .22 & .73 \\ & & & & & & & \\ \text { DEC } & 9 & 0835 & 160 & 19 & 9 & .46 & .70 \\ \text { DEC } & 12 & 1240 & 140 & 32 & 108 & 9.3 & 1.2 \\ \text { OEC } & 16 & 0925 & 145 & 17 & 6 & .28 & .62 \\ \text { OEC } & 30 & 0820 & 155 & 16 & 38 & 1.6 & .59 \\ \text { JAN } & 13 & 1020 & 155 & 14 & 6 & .23 & .38 \\ \text { JAN } & 21 & 0845 & 155 & 13 & 3 & .11 & .48 \\ \text { FEB } & 3 & 0820 & 170 & 13 & 4 & .14 & .48 \\ \text { FEB } & 10 & 0845 & 160 & 12 & 1 & .03 & .44 \\ \text { FEB } & 18 & 0935 & 160 & 12 & 1 & .03 & .44 \\ \text { FEB } & 24 & 0755 & 170 & 8.4 & 2 & .05 & .31\end{array}$




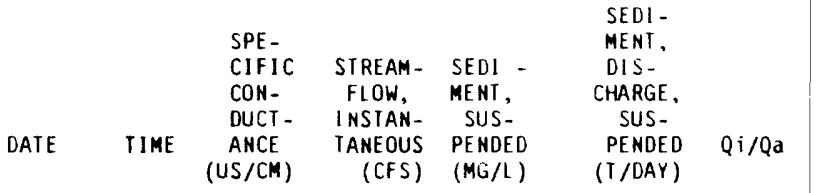

WATER YEAR OCTO8ER 1985 TO SEPIEMBER 1986-Cont inued

\begin{tabular}{|c|c|c|c|c|c|c|c|}
\hline $\begin{array}{l}\text { MAR } \\
\text { MAR } \\
\text { MAR } \\
\text { APR } \\
\text { APR }\end{array}$ & $\begin{array}{l}17 \\
24 \\
31 \\
14 \\
28\end{array}$ & $\begin{array}{l}0910 \\
0835 \\
0835 \\
0855 \\
1045\end{array}$ & $\begin{array}{l}170 \\
180 \\
160 \\
170 \\
150\end{array}$ & $\begin{array}{l}7.0 \\
7.0 \\
11 \\
9.4 \\
16\end{array}$ & $\begin{array}{r}2 \\
4 \\
9 \\
3 \\
33\end{array}$ & $\begin{array}{r}0.04 \\
.08 \\
.27 \\
.08 \\
1.4\end{array}$ & $\begin{array}{r}0.26 \\
.26 \\
.40 \\
.34 \\
.59\end{array}$ \\
\hline $\begin{array}{l}\text { MAY } \\
\text { MAY } \\
\text { MAY } \\
\text { MAY } \\
\text { MAY }\end{array}$ & $\begin{array}{l}1 \\
5 \\
8 \\
8 \\
8\end{array}$ & $\begin{array}{l}0855 \\
0850 \\
1600 \\
1605 \\
1610\end{array}$ & $\begin{array}{l}140 \\
170 \\
160 \\
110 \\
100\end{array}$ & $\begin{array}{r}20 \\
12 \\
200 \\
500 \\
803\end{array}$ & $\begin{array}{r}33 \\
6 \\
1,630 \\
2,140 \\
2,330\end{array}$ & $\begin{array}{r}1.8 \\
.19 \\
880 \\
2,890 \\
5,050\end{array}$ & $\begin{array}{l}.73 \\
1.9^{.44} \\
30\end{array}$ \\
\hline $\begin{array}{l}\text { MAY } \\
\text { MAY } \\
\text { MAY } \\
\text { MAY } \\
\text { MAY }\end{array}$ & $\begin{array}{l}8 \\
8 \\
8 \\
8 \\
8\end{array}$ & $\begin{array}{l}1620 \\
1630 \\
1640 \\
1650 \\
1700\end{array}$ & $\begin{array}{r}100 \\
100 \\
100 \\
90 \\
90\end{array}$ & $\begin{array}{l}1,310 \\
1,780 \\
1,710 \\
1,660 \\
1,610\end{array}$ & $\begin{array}{l}3,680 \\
3,750 \\
3,350 \\
3,150 \\
3,110\end{array}$ & $\begin{array}{l}13,000 \\
18,000 \\
15,500 \\
14,100 \\
13,500\end{array}$ & $\begin{array}{l}49 \\
66 \\
64 \\
62 \\
60\end{array}$ \\
\hline $\begin{array}{l}\text { MAY } \\
\text { MAY } \\
\text { MAY } \\
\text { MAY } \\
\text { MAY }\end{array}$ & $\begin{array}{l}8 \\
8 \\
8 \\
8 \\
8\end{array}$ & $\begin{array}{l}1715 \\
1730 \\
1745 \\
1800 \\
1815\end{array}$ & $\begin{array}{l}90 \\
90 \\
90 \\
90 \\
90\end{array}$ & $\begin{array}{r}1,090 \\
888 \\
614 \\
445 \\
374\end{array}$ & $\begin{array}{r}2.380 \\
2,230 \\
1,510 \\
1,110 \\
988\end{array}$ & $\begin{array}{r}7,000 \\
5,350 \\
2,500 \\
1,330 \\
998\end{array}$ & $\begin{array}{l}40 \\
33 \\
23 \\
17 \\
14\end{array}$ \\
\hline $\begin{array}{l}\text { MAY } \\
\text { MAY } \\
\text { MAY } \\
\text { MAY } \\
\text { MAY }\end{array}$ & $\begin{array}{l}8 \\
8 \\
8 \\
8 \\
8\end{array}$ & $\begin{array}{l}1830 \\
1845 \\
1900 \\
1915 \\
1945\end{array}$ & $\begin{array}{l}100 \\
100 \\
110 \\
110 \\
130\end{array}$ & $\begin{array}{l}330 \\
289 \\
271 \\
257 \\
209\end{array}$ & $\begin{array}{l}814 \\
691 \\
604 \\
618 \\
412\end{array}$ & $\begin{array}{l}725 \\
539 \\
442 \\
429 \\
232\end{array}$ & $\begin{array}{l}12 \\
11 \\
10 \\
9.5 \\
7.8\end{array}$ \\
\hline $\begin{array}{l}\text { MAY } \\
\text { MAY } \\
\text { MAY } \\
\text { MAY } \\
\text { MAY }\end{array}$ & $\begin{array}{l}8 \\
8 \\
8 \\
8 \\
8\end{array}$ & $\begin{array}{l}2015 \\
2045 \\
2115 \\
2145 \\
2215\end{array}$ & $\begin{array}{l}120 \\
130 \\
130 \\
130 \\
140\end{array}$ & $\begin{array}{l}181 \\
152 \\
125 \\
113 \\
104\end{array}$ & $\begin{array}{l}303 \\
260 \\
213 \\
181 \\
170\end{array}$ & $\begin{array}{r}148 \\
107 \\
72 \\
55 \\
48\end{array}$ & $\begin{array}{l}6.7 \\
5.6 \\
4.6 \\
4.2 \\
3.9\end{array}$ \\
\hline $\begin{array}{l}\text { MAY } \\
\text { MAY } \\
\text { MAY } \\
\text { MAY } \\
\text { MAY }\end{array}$ & $\begin{array}{l}8 \\
13 \\
13 \\
13 \\
13\end{array}$ & $\begin{array}{l}2245 \\
0735 \\
0740 \\
0745 \\
0755\end{array}$ & $\begin{array}{r}140 \\
125 \\
105 \\
80 \\
75\end{array}$ & $\begin{array}{r}95 \\
205 \\
263 \\
429 \\
963\end{array}$ & $\begin{array}{r}153 \\
745 \\
2,040 \\
3,420 \\
10,200\end{array}$ & $\begin{array}{r}39 \\
412 \\
1,450 \\
3,960 \\
26,500\end{array}$ & $\begin{array}{l}3.5 \\
7.5 \\
9.6 \\
16 \\
35\end{array}$ \\
\hline $\begin{array}{l}\text { MAY } \\
\text { MAY } \\
\text { MAY } \\
\text { MAY } \\
\text { MAY }\end{array}$ & $\begin{array}{l}13 \\
13 \\
13 \\
13 \\
13\end{array}$ & $\begin{array}{l}0805 \\
0815 \\
0825 \\
0835 \\
0850\end{array}$ & $\begin{array}{l}75 \\
75 \\
50 \\
60 \\
50\end{array}$ & $\begin{array}{l}1,700 \\
1,990 \\
1,970 \\
1,660 \\
1,530\end{array}$ & $\begin{array}{r}20,600 \\
16,800 \\
9,840 \\
13,000 \\
6,430\end{array}$ & $\begin{array}{l}94,500 \\
90,300 \\
52,300 \\
58,300 \\
\angle 6,600\end{array}$ & $\begin{array}{l}62 \\
73 \\
72 \\
61 \\
56\end{array}$ \\
\hline $\begin{array}{l}\text { MAY } \\
\text { MAY } \\
\text { MAY } \\
\text { MAY } \\
\text { MAY }\end{array}$ & $\begin{array}{l}13 \\
13 \\
13 \\
13 \\
13\end{array}$ & $\begin{array}{l}0905 \\
0920 \\
0935 \\
0950 \\
1005\end{array}$ & $\begin{array}{l}50 \\
55 \\
60 \\
60 \\
70\end{array}$ & $\begin{array}{r}1,140 \\
977 \\
733 \\
524 \\
437\end{array}$ & $\begin{array}{l}3,980 \\
2,880 \\
3,170 \\
1,580 \\
1,640\end{array}$ & $\begin{array}{r}12,200 \\
7,600 \\
6,300 \\
2.240 \\
1,940\end{array}$ & $\begin{array}{l}42 \\
36 \\
27 \\
19 \\
16\end{array}$ \\
\hline $\begin{array}{l}\text { MAY } \\
\text { MAY } \\
\text { MAY } \\
\text { MAY } \\
\text { MAY }\end{array}$ & $\begin{array}{l}13 \\
13 \\
13 \\
13 \\
13\end{array}$ & $\begin{array}{l}1020 \\
1035 \\
1105 \\
1135 \\
1205\end{array}$ & $\begin{array}{l}70 \\
70 \\
70 \\
80 \\
90\end{array}$ & $\begin{array}{l}362 \\
326 \\
271 \\
231 \\
206\end{array}$ & $\begin{array}{r}1,100 \\
808 \\
612 \\
396 \\
308\end{array}$ & $\begin{array}{r}1,080 \\
711 \\
448 \\
247 \\
171\end{array}$ & $\begin{array}{r}13 \\
12 \\
9.9 \\
8.5 \\
7.6\end{array}$ \\
\hline $\begin{array}{l}\text { MAY } \\
\text { MAY } \\
\text { MAY } \\
\text { MAY } \\
\text { MAY }\end{array}$ & $\begin{array}{l}13 \\
13 \\
13 \\
13 \\
13\end{array}$ & $\begin{array}{l}1235 \\
1305 \\
1335 \\
1405 \\
1435\end{array}$ & $\begin{array}{r}90 \\
100 \\
100 \\
100 \\
100\end{array}$ & $\begin{array}{l}181 \\
166 \\
146 \\
128 \\
116\end{array}$ & $\begin{array}{l}258 \\
217 \\
176 \\
157 \\
141\end{array}$ & $\begin{array}{r}126 \\
97 \\
69 \\
54 \\
47\end{array}$ & $\begin{array}{l}6.6 \\
6.1 \\
5.3 \\
4.7 \\
4.2\end{array}$ \\
\hline $\begin{array}{l}\text { MAY } \\
\text { MAY } \\
\text { MAY } \\
\text { MAY } \\
\text { MAY }\end{array}$ & $\begin{array}{l}18 \\
18 \\
18 \\
18 \\
18\end{array}$ & $\begin{array}{l}0115 \\
0120 \\
0125 \\
0135 \\
0145\end{array}$ & $\begin{array}{r}110 \\
110 \\
100 \\
80 \\
85\end{array}$ & $\begin{array}{l}200 \\
210 \\
230 \\
315 \\
445\end{array}$ & $\begin{array}{r}515 \\
920 \\
442 \\
1,180 \\
2,080\end{array}$ & $\begin{array}{r}278 \\
522 \\
274 \\
1,000 \\
2,500\end{array}$ & $\begin{array}{l}7.3 \\
7.7 \\
8.4 \\
12 \\
16\end{array}$ \\
\hline
\end{tabular}

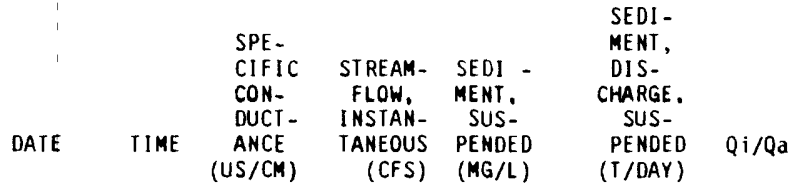

WATER YEAR OCTOBER 1985 TO SEPTEMBER 1986

\begin{tabular}{|c|c|c|c|c|c|c|c|}
\hline $\begin{array}{l}\text { MAY } \\
\text { MAY } \\
\text { MAY } \\
\text { MAY } \\
\text { MAY }\end{array}$ & $\begin{array}{l}18 \\
18 \\
18 \\
18 \\
18\end{array}$ & $\begin{array}{l}0155 \\
0205 \\
0215 \\
0230 \\
0245\end{array}$ & $\begin{array}{l}80 \\
75 \\
70 \\
70 \\
70\end{array}$ & $\begin{array}{l}434 \\
381 \\
318 \\
274 \\
234\end{array}$ & $\begin{array}{l}1,630 \\
1,540 \\
1,430 \\
1,350 \\
1,340\end{array}$ & $\begin{array}{r}1,910 \\
1,580 \\
1,230 \\
999 \\
847\end{array}$ & $\begin{array}{c}16 \\
14 \\
12 \\
10 \\
8.6\end{array}$ \\
\hline $\begin{array}{l}\text { MAY } \\
\text { MAY } \\
\text { MAY } \\
\text { MAY } \\
\text { MAY }\end{array}$ & $\begin{array}{l}1 B \\
18 \\
18 \\
18 \\
18\end{array}$ & $\begin{array}{l}0300 \\
0315 \\
0330 \\
0345 \\
0400\end{array}$ & $\begin{array}{l}70 \\
70 \\
70 \\
70 \\
70\end{array}$ & $\begin{array}{l}209 \\
187 \\
157 \\
141 \\
130\end{array}$ & $\begin{array}{r}1,260 \\
969 \\
837 \\
681 \\
612\end{array}$ & $\begin{array}{l}711 \\
489 \\
351 \\
259 \\
215\end{array}$ & $\begin{array}{l}7.7 \\
6.8 \\
5.8 \\
5.2 \\
4.8\end{array}$ \\
\hline $\begin{array}{l}\text { MAY } \\
\text { MAY } \\
\text { MAY } \\
\text { MAY } \\
\text { MAY }\end{array}$ & $\begin{array}{l}18 \\
18 \\
18 \\
18 \\
18\end{array}$ & $\begin{array}{l}0415 \\
0445 \\
0515 \\
0545 \\
0615\end{array}$ & $\begin{array}{r}70 \\
80 \\
80 \\
90 \\
100\end{array}$ & $\begin{array}{r}128 \\
113 \\
107 \\
97 \\
87\end{array}$ & $\begin{array}{l}417 \\
515 \\
266 \\
330 \\
219\end{array}$ & $\begin{array}{r}144 \\
157 \\
77 \\
86 \\
51\end{array}$ & $\begin{array}{l}4.7 \\
4.1 \\
3.9 \\
3.6 \\
3.2\end{array}$ \\
\hline $\begin{array}{l}\text { MAY } \\
\text { MAY } \\
\text { MAY } \\
\text { MAY } \\
\text { MAY }\end{array}$ & $\begin{array}{l}18 \\
18 \\
18 \\
18 \\
31\end{array}$ & $\begin{array}{l}0645 \\
0715 \\
0745 \\
0815 \\
0415\end{array}$ & $\begin{array}{l}100 \\
100 \\
100 \\
100 \\
145\end{array}$ & $\begin{array}{r}82 \\
75 \\
70 \\
66 \\
190\end{array}$ & $\begin{array}{r}180 \\
157 \\
143 \\
124 \\
1,160\end{array}$ & $\begin{array}{r}40 \\
32 \\
27 \\
22 \\
595\end{array}$ & $\begin{array}{l}3.0 \\
2.7 \\
2.6 \\
2.4 \\
7.0\end{array}$ \\
\hline $\begin{array}{l}\text { MAY } \\
\text { MAY } \\
\text { MAY } \\
\text { MAY } \\
\text { MAY }\end{array}$ & $\begin{array}{l}31 \\
31 \\
31 \\
31 \\
31\end{array}$ & $\begin{array}{l}0420 \\
0425 \\
0435 \\
0445 \\
0455\end{array}$ & $\begin{array}{l}135 \\
125 \\
100 \\
105 \\
105\end{array}$ & $\begin{array}{l}200 \\
210 \\
230 \\
250 \\
270\end{array}$ & $\begin{array}{l}1,030 \\
1,080 \\
1,090 \\
1,280 \\
1,700\end{array}$ & $\begin{array}{r}556 \\
612 \\
677 \\
864 \\
1.240\end{array}$ & $\begin{array}{l}7.3 \\
7.7 \\
8.4 \\
9.2 \\
9.9\end{array}$ \\
\hline $\begin{array}{l}\text { MAY } \\
\text { MAY } \\
\text { MAY } \\
\text { MAY } \\
\text { MAY }\end{array}$ & $\begin{array}{l}31 \\
31 \\
31 \\
31 \\
31\end{array}$ & $\begin{array}{l}0505 \\
0515 \\
0530 \\
0545 \\
0600\end{array}$ & $\begin{array}{r}105 \\
100 \\
90 \\
85 \\
80\end{array}$ & $\begin{array}{l}290 \\
234 \\
197 \\
169 \\
149\end{array}$ & $\begin{array}{l}1,500 \\
1,390 \\
1,980 \\
1,960 \\
1,620\end{array}$ & $\begin{array}{r}1,170 \\
878 \\
1,050 \\
894 \\
652\end{array}$ & $\begin{array}{r}11 \\
8.6 \\
7.2 \\
6.2 \\
5.5\end{array}$ \\
\hline $\begin{array}{l}\text { MAY } \\
\text { MAY } \\
\text { MAY } \\
\text { MAY } \\
\text { MAY }\end{array}$ & $\begin{array}{l}31 \\
31 \\
31 \\
31 \\
31\end{array}$ & $\begin{array}{l}0615 \\
0630 \\
0645 \\
0700 \\
0715\end{array}$ & $\begin{array}{l}80 \\
80 \\
80 \\
80 \\
80\end{array}$ & $\begin{array}{r}133 \\
125 \\
116 \\
107 \\
97\end{array}$ & $\begin{array}{r}1,460 \\
1,330 \\
1,220 \\
1,100 \\
938\end{array}$ & $\begin{array}{l}524 \\
449 \\
382 \\
318 \\
246\end{array}$ & $\begin{array}{l}4.9 \\
4.6 \\
4.2 \\
3.9 \\
3.6\end{array}$ \\
\hline $\begin{array}{l}\text { MAY } \\
\text { MAY } \\
\text { MAY } \\
\text { MAY } \\
\text { MAY }\end{array}$ & $\begin{array}{l}31 \\
31 \\
31 \\
31 \\
31\end{array}$ & $\begin{array}{l}0745 \\
0815 \\
0845 \\
0915 \\
0945\end{array}$ & $\begin{array}{l}85 \\
85 \\
90 \\
90 \\
90\end{array}$ & $\begin{array}{l}79 \\
64 \\
54 \\
49 \\
46\end{array}$ & $\begin{array}{l}901 \\
728 \\
552 \\
440 \\
391\end{array}$ & $\begin{array}{r}192 \\
126 \\
80 \\
58 \\
49\end{array}$ & $\begin{array}{l}2.9 \\
2.3 \\
2.0 \\
1.8 \\
1.7\end{array}$ \\
\hline $\begin{array}{l}\text { MAY } \\
\text { MAY } \\
\text { MAY } \\
\text { JUNE } \\
\text { JUNE }\end{array}$ & $\begin{array}{l}31 \\
31 \\
31 \\
10 \\
10\end{array}$ & $\begin{array}{l}1015 \\
1045 \\
1115 \\
0130 \\
0135\end{array}$ & $\begin{array}{l}100 \\
110 \\
110 \\
150 \\
110\end{array}$ & $\begin{array}{r}39 \\
35 \\
33 \\
200 \\
375\end{array}$ & $\begin{array}{r}324 \\
243 \\
238 \\
2,290 \\
4,090\end{array}$ & $\begin{array}{r}34 \\
23 \\
21 \\
1,240 \\
4,140\end{array}$ & $\begin{array}{r}1.4 \\
1.3 \\
1.2 \\
7.3 \\
14\end{array}$ \\
\hline $\begin{array}{l}\text { JUNE } \\
\text { JUNE } \\
\text { JUNE } \\
\text { JUNE } \\
\text { JUNE }\end{array}$ & $\begin{array}{l}10 \\
10 \\
10 \\
10 \\
10\end{array}$ & $\begin{array}{l}0140 \\
0150 \\
0200 \\
0210 \\
0220\end{array}$ & $\begin{array}{r}110 \\
110 \\
110 \\
90 \\
80\end{array}$ & $\begin{array}{l}496 \\
718 \\
916 \\
985 \\
920\end{array}$ & $\begin{array}{r}5,020 \\
5,820 \\
14,600 \\
9,940 \\
9,840\end{array}$ & $\begin{array}{r}6,720 \\
11,300 \\
36,100 \\
26,400 \\
24,400\end{array}$ & $\begin{array}{l}18 \\
26 \\
34 \\
36 \\
34\end{array}$ \\
\hline $\begin{array}{l}\text { JUNE } \\
\text { JUNE } \\
\text { JUNE } \\
\text { JUNE } \\
\text { JUNE }\end{array}$ & $\begin{array}{l}10 \\
10 \\
10 \\
10 \\
10\end{array}$ & $\begin{array}{l}0230 \\
0245 \\
0300 \\
0315 \\
0330\end{array}$ & $\begin{array}{l}80 \\
80 \\
80 \\
80 \\
80\end{array}$ & $\begin{array}{l}728 \\
501 \\
374 \\
300 \\
254\end{array}$ & $\begin{array}{l}9,230 \\
7,870 \\
5,740 \\
5,060 \\
3,690\end{array}$ & $\begin{array}{r}18,100 \\
10,600 \\
5,800 \\
4,100 \\
2,530\end{array}$ & $\begin{array}{c}27 \\
18 \\
14 \\
11 \\
9.3\end{array}$ \\
\hline $\begin{array}{l}\text { JUNE } \\
\text { JUNE } \\
\text { JUNE } \\
\text { JUNE } \\
\text { JUNE }\end{array}$ & $\begin{array}{l}10 \\
10 \\
10 \\
10 \\
10\end{array}$ & $\begin{array}{l}0345 \\
0400 \\
0415 \\
0430 \\
0500\end{array}$ & $\begin{array}{l}80 \\
80 \\
90 \\
90 \\
95\end{array}$ & $\begin{array}{l}234 \\
197 \\
163 \\
157 \\
130\end{array}$ & $\begin{array}{l}3,220 \\
2,520 \\
2,250 \\
1,780 \\
1,310\end{array}$ & $\begin{array}{r}2,030 \\
1,340 \\
990 \\
754 \\
460\end{array}$ & $\begin{array}{l}8.6 \\
7.2 \\
6.0 \\
5.6 \\
4.8\end{array}$ \\
\hline
\end{tabular}


Iable 8. Suspended-sediment data for samples collected at station Rio Turabo at Borinquen

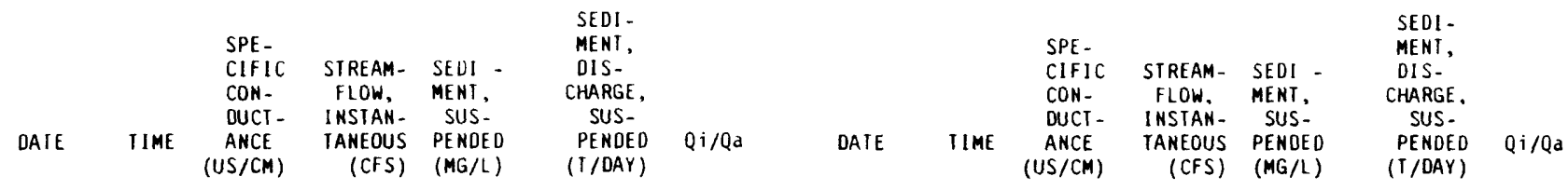

WATER YEAR OCTOBER 1985 TD SEPTEMBER 1986 -Cont inued

WATER YEAR DCTOBER 1985 TD SEPTEMBER 1986

\begin{tabular}{|c|c|c|c|c|c|c|c|c|c|c|c|c|c|c|c|}
\hline $\begin{array}{l}\text { JUNE } \\
\text { JUNE } \\
\text { JUNE } \\
\text { JUNE } \\
\text { JUNE }\end{array}$ & $\begin{array}{l}10 \\
10 \\
10 \\
10 \\
10\end{array}$ & $\begin{array}{l}0530 \\
0600 \\
0630 \\
0700 \\
0730\end{array}$ & $\begin{array}{r}95 \\
100 \\
110 \\
110 \\
115\end{array}$ & $\begin{array}{r}116 \\
104 \\
104 \\
97 \\
100\end{array}$ & $\begin{array}{r}1,140 \\
777 \\
572 \\
462 \\
371\end{array}$ & $\begin{array}{l}357 \\
218 \\
161 \\
121 \\
100\end{array}$ & $\begin{array}{l}4.2 \\
3.8 \\
3.8 \\
3.6 \\
3.7\end{array}$ & $\begin{array}{l}\text { AUG } \\
\text { AUG } \\
\text { AUG } \\
\text { AUG } \\
\text { AUG }\end{array}$ & $\begin{array}{l}29 \\
29 \\
29 \\
29 \\
29\end{array}$ & $\begin{array}{l}0930 \\
0945 \\
1000 \\
1015 \\
1030\end{array}$ & $\begin{array}{l}75 \\
80 \\
80 \\
80 \\
80\end{array}$ & $\begin{array}{l}215 \\
200 \\
181 \\
166 \\
154\end{array}$ & $\begin{array}{l}790 \\
737 \\
617 \\
505 \\
403\end{array}$ & $\begin{array}{l}459 \\
398 \\
302 \\
226 \\
168\end{array}$ & $\begin{array}{l}7.9 \\
7.3 \\
6.6 \\
6.1 \\
5.6\end{array}$ \\
\hline $\begin{array}{l}\text { JUNE } \\
\text { JUNE } \\
\text { JUNE } \\
\text { JUNE } \\
\text { JULY }\end{array}$ & $\begin{array}{l}10 \\
10 \\
10 \\
23 \\
1\end{array}$ & $\begin{array}{l}0800 \\
0830 \\
0855 \\
0830 \\
0840\end{array}$ & $\begin{array}{l}115 \\
115 \\
170 \\
170 \\
180\end{array}$ & $\begin{array}{l}\text { B4 } \\
72 \\
20 \\
15 \\
11\end{array}$ & $\begin{array}{r}388 \\
386 \\
7 \\
2 \\
3\end{array}$ & $\begin{array}{l}88 \\
75 \\
.38 \\
.08 \\
.09\end{array}$ & $\begin{array}{l}3.1 \\
2.6 \\
.73 \\
.55 \\
.40\end{array}$ & $\begin{array}{l}A \cup G \\
A \cup G \\
A \cup G \\
A \cup G \\
A \cup G\end{array}$ & $\begin{array}{l}29 \\
29 \\
29 \\
29 \\
29\end{array}$ & $\begin{array}{l}1045 \\
1100 \\
1115 \\
1130 \\
1200\end{array}$ & $\begin{array}{r}90 \\
90 \\
90 \\
100 \\
100\end{array}$ & $\begin{array}{l}160 \\
149 \\
138 \\
125 \\
121\end{array}$ & $\begin{array}{l}347 \\
284 \\
253 \\
222 \\
234\end{array}$ & $\begin{array}{r}150 \\
114 \\
94 \\
75 \\
76\end{array}$ & $\begin{array}{l}5.9 \\
5.5 \\
5.1 \\
4.6 \\
4.4\end{array}$ \\
\hline $\begin{array}{l}\text { JULY } \\
\text { AUG } \\
\text { AUG } \\
\text { AUG } \\
\text { AUG }\end{array}$ & $\begin{array}{r}14 \\
4 \\
12 \\
19 \\
28\end{array}$ & $\begin{array}{l}0915 \\
0900 \\
0845 \\
0800 \\
1930\end{array}$ & $\begin{array}{l}180 \\
190 \\
170 \\
190 \\
120\end{array}$ & $\begin{array}{r}13 \\
17 \\
24 \\
20 \\
200\end{array}$ & $\begin{array}{r}4 \\
2 \\
14 \\
3 \\
2,110\end{array}$ & $1,140^{.16} .09$ & $\begin{array}{l}.48 \\
.62 \\
.88 \\
.73 \\
7.3\end{array}$ & $\begin{array}{l}A \cup G \\
A \cup G \\
A \cup G \\
A \cup G \\
A \cup G\end{array}$ & $\begin{array}{l}29 \\
29 \\
29 \\
29 \\
29\end{array}$ & $\begin{array}{l}1230 \\
1300 \\
1330 \\
1400 \\
1430\end{array}$ & $\begin{array}{l}100 \\
100 \\
100 \\
105 \\
105\end{array}$ & $\begin{array}{r}113 \\
100 \\
92 \\
79 \\
70\end{array}$ & $\begin{array}{l}197 \\
169 \\
150 \\
128 \\
111\end{array}$ & $\begin{array}{l}60 \\
46 \\
37 \\
27 \\
21\end{array}$ & $\begin{array}{l}4.1 \\
3.7 \\
3.4 \\
2.9 \\
2.6\end{array}$ \\
\hline $\begin{array}{l}\text { AUG } \\
\text { AUG } \\
\text { AUG } \\
\text { AUG } \\
\text { AUG }\end{array}$ & $\begin{array}{l}28 \\
28 \\
28 \\
28 \\
28\end{array}$ & $\begin{array}{l}1935 \\
1940 \\
1950 \\
2000 \\
2010\end{array}$ & $\begin{array}{l}120 \\
120 \\
120 \\
110 \\
110\end{array}$ & $\begin{array}{l}202 \\
204 \\
205 \\
203 \\
199\end{array}$ & $\begin{array}{l}1,050 \\
1,010 \\
1,120 \\
1,160 \\
1,280\end{array}$ & $\begin{array}{l}573 \\
556 \\
620 \\
636 \\
688\end{array}$ & $\begin{array}{l}7.4 \\
7.5 \\
7.5 \\
7.5 \\
7.3\end{array}$ & $\begin{array}{l}\text { AUG } \\
\text { AUG } \\
\text { SEPT } \\
\text { SEPT } \\
\text { SEPT }\end{array}$ & $\begin{array}{l}29 \\
29 \\
22 \\
24 \\
24\end{array}$ & $\begin{array}{l}1500 \\
1530 \\
1015 \\
1115 \\
1120\end{array}$ & $\begin{array}{l}110 \\
110 \\
170 \\
140 \\
140\end{array}$ & $\begin{array}{c}68 \\
66 \\
6.6 \\
201 \\
168\end{array}$ & $\begin{array}{r}108 \\
110 \\
7 \\
585 \\
701\end{array}$ & $\begin{array}{l}20 \\
20 \\
318^{.12} \\
318\end{array}$ & $\begin{array}{l}2.5 \\
2.4 \\
.24 \\
7.4 \\
6.2\end{array}$ \\
\hline $\begin{array}{l}\text { AUG } \\
\text { AUG } \\
\text { AUG } \\
\text { AUG } \\
\text { AUG }\end{array}$ & $\begin{array}{l}28 \\
28 \\
28 \\
28 \\
28\end{array}$ & $\begin{array}{l}2020 \\
2030 \\
2045 \\
2100 \\
2115\end{array}$ & $\begin{array}{l}110 \\
110 \\
100 \\
100 \\
100\end{array}$ & $\begin{array}{l}222 \\
271 \\
267 \\
289 \\
289\end{array}$ & $\begin{array}{l}1,250 \\
1,190 \\
1,200 \\
1,050 \\
1,100\end{array}$ & $\begin{array}{l}749 \\
871 \\
865 \\
819 \\
858\end{array}$ & $\begin{array}{l}8.1 \\
9.9 \\
9.8 \\
11 \\
11\end{array}$ & $\begin{array}{l}\text { SEPT } \\
\text { SEPT } \\
\text { SEPT } \\
\text { SEPT } \\
\text { SEPT }\end{array}$ & $\begin{array}{l}24 \\
24 \\
24 \\
24 \\
24\end{array}$ & $\begin{array}{l}1125 \\
1135 \\
1145 \\
1155 \\
1205\end{array}$ & $\begin{array}{l}140 \\
110 \\
110 \\
110 \\
110\end{array}$ & $\begin{array}{l}150 \\
178 \\
261 \\
117 \\
190\end{array}$ & $\begin{array}{r}717 \\
1,030 \\
873 \\
740 \\
1,010\end{array}$ & $\begin{array}{l}290 \\
495 \\
615 \\
234 \\
518\end{array}$ & $\begin{array}{l}5.5 \\
6.5 \\
9.6 \\
4.3 \\
7.0\end{array}$ \\
\hline $\begin{array}{l}A \cup G \\
A \cup G \\
A \cup G \\
A \cup G \\
A \cup G\end{array}$ & $\begin{array}{l}28 \\
28 \\
28 \\
28 \\
2 B\end{array}$ & $\begin{array}{l}2130 \\
2145 \\
2200 \\
2215 \\
2230\end{array}$ & $\begin{array}{r}100 \\
100 \\
100 \\
95 \\
95\end{array}$ & $\begin{array}{l}267 \\
234 \\
224 \\
212 \\
271\end{array}$ & $\begin{array}{l}1,370 \\
2,100 \\
2,290 \\
1,900 \\
1,520\end{array}$ & $\begin{array}{r}988 \\
1,330 \\
1,380 \\
1,090 \\
1,110\end{array}$ & $\begin{array}{l}9.8 \\
8.6 \\
8.2 \\
7.8 \\
9.9\end{array}$ & $\begin{array}{l}\text { SEPT } \\
\text { SEPT } \\
\text { SEPT } \\
\text { SEPT } \\
\text { SEPT }\end{array}$ & $\begin{array}{l}24 \\
24 \\
24 \\
24 \\
24\end{array}$ & $\begin{array}{l}1215 \\
1230 \\
1245 \\
1300 \\
1315\end{array}$ & $\begin{array}{l}120 \\
120 \\
110 \\
110 \\
110\end{array}$ & $\begin{array}{l}175 \\
160 \\
146 \\
118 \\
.116\end{array}$ & $\begin{array}{r}964 \\
994 \\
1,170 \\
1,290 \\
1,350\end{array}$ & $\begin{array}{l}455 \\
429 \\
461 \\
411 \\
423\end{array}$ & $\begin{array}{l}6.4 \\
5.9 \\
5.3 \\
4.3 \\
4.2\end{array}$ \\
\hline $\begin{array}{l}\text { AUG } \\
\text { AUG } \\
\text { AUG } \\
\text { AUG } \\
\text { AUG }\end{array}$ & $\begin{array}{l}28 \\
28 \\
28 \\
29 \\
29\end{array}$ & $\begin{array}{l}2300 \\
2330 \\
2400 \\
0030 \\
0100\end{array}$ & $\begin{array}{l}90 \\
90 \\
90 \\
90 \\
80\end{array}$ & $\begin{array}{l}362 \\
296 \\
244 \\
257 \\
585\end{array}$ & $\begin{array}{l}1,610 \\
1,900 \\
3,440 \\
4,280 \\
4,000\end{array}$ & $\begin{array}{l}1,570 \\
1,520 \\
2,270 \\
2,970 \\
6,320\end{array}$ & $\begin{array}{l}13 \\
11 \\
8.9 \\
9.4 \\
21\end{array}$ & $\begin{array}{l}\text { SEPT } \\
\text { SEPT } \\
\text { SEPT } \\
\text { SEPT } \\
\text { SEPT }\end{array}$ & $\begin{array}{l}24 \\
24 \\
24 \\
24 \\
24\end{array}$ & $\begin{array}{l}1330 \\
1345 \\
1400 \\
1415 \\
1445\end{array}$ & $\begin{array}{l}110 \\
110 \\
110 \\
110 \\
110\end{array}$ & $\begin{array}{r}100 \\
79 \\
77 \\
92 \\
113\end{array}$ & $\begin{array}{r}1,250 \\
1,170 \\
1,080 \\
976 \\
922\end{array}$ & $\begin{array}{l}338 \\
250 \\
224 \\
242 \\
281\end{array}$ & $\begin{array}{l}3.7 \\
2.9 \\
2.8 \\
3.4 \\
4.1\end{array}$ \\
\hline $\begin{array}{l}\text { AUG } \\
\text { AUG } \\
\text { AUG } \\
\text { AUG } \\
\text { AUG }\end{array}$ & $\begin{array}{l}29 \\
29 \\
29 \\
29 \\
29\end{array}$ & $\begin{array}{l}0130 \\
0200 \\
0230 \\
0830 \\
0835\end{array}$ & $\begin{array}{l}80 \\
80 \\
80 \\
70 \\
70\end{array}$ & $\begin{array}{r}1,570 \\
966 \\
600 \\
350 \\
341\end{array}$ & $\begin{array}{l}4,020 \\
3,030 \\
2,370 \\
2,320 \\
1,450\end{array}$ & $\begin{array}{r}17,000 \\
7,900 \\
3,840 \\
2,190 \\
1,340\end{array}$ & $\begin{array}{l}58 \\
35 \\
22 \\
13 \\
12\end{array}$ & $\begin{array}{l}\text { SEPT } \\
\text { SEPT } \\
\text { SEPT } \\
\text { SEPT } \\
\text { SEPT }\end{array}$ & $\begin{array}{l}24 \\
24 \\
24 \\
24 \\
24\end{array}$ & $\begin{array}{l}1515 \\
1545 \\
1615 \\
1645 \\
1715\end{array}$ & $\begin{array}{l}110 \\
110 \\
110 \\
110 \\
110\end{array}$ & $\begin{array}{l}118 \\
111 \\
163 \\
136 \\
111\end{array}$ & $\begin{array}{l}964 \\
822 \\
743 \\
854 \\
677\end{array}$ & $\begin{array}{l}307 \\
246 \\
327 \\
314 \\
203\end{array}$ & $\begin{array}{l}4.3 \\
4.1 \\
6.0 \\
5.0 \\
4.1\end{array}$ \\
\hline $\begin{array}{l}\text { AUG } \\
\text { AUG } \\
\text { AUG } \\
\text { AUG } \\
\text { AUG }\end{array}$ & $\begin{array}{l}29 \\
29 \\
29 \\
29 \\
29\end{array}$ & $\begin{array}{l}0840 \\
0850 \\
0900 \\
0910 \\
0920\end{array}$ & $\begin{array}{l}70 \\
70 \\
70 \\
70 \\
75\end{array}$ & $\begin{array}{l}332 \\
305 \\
271 \\
250 \\
249\end{array}$ & $\begin{array}{r}1,020 \\
963 \\
886 \\
824 \\
819\end{array}$ & $\begin{array}{l}914 \\
793 \\
648 \\
556 \\
551\end{array}$ & $\begin{array}{l}12 \\
11 \\
9.9 \\
9.2 \\
9.1\end{array}$ & $\begin{array}{l}\text { SEPT } \\
\text { SEPT }\end{array}$ & $\begin{array}{l}24 \\
24\end{array}$ & $\begin{array}{l}1745 \\
1815\end{array}$ & $\begin{array}{l}110 \\
110\end{array}$ & $\begin{array}{l}84 \\
64\end{array}$ & $\begin{array}{l}471 \\
382\end{array}$ & $\begin{array}{r}107 \\
66\end{array}$ & $\begin{array}{l}3.1 \\
2.3\end{array}$ \\
\hline
\end{tabular}




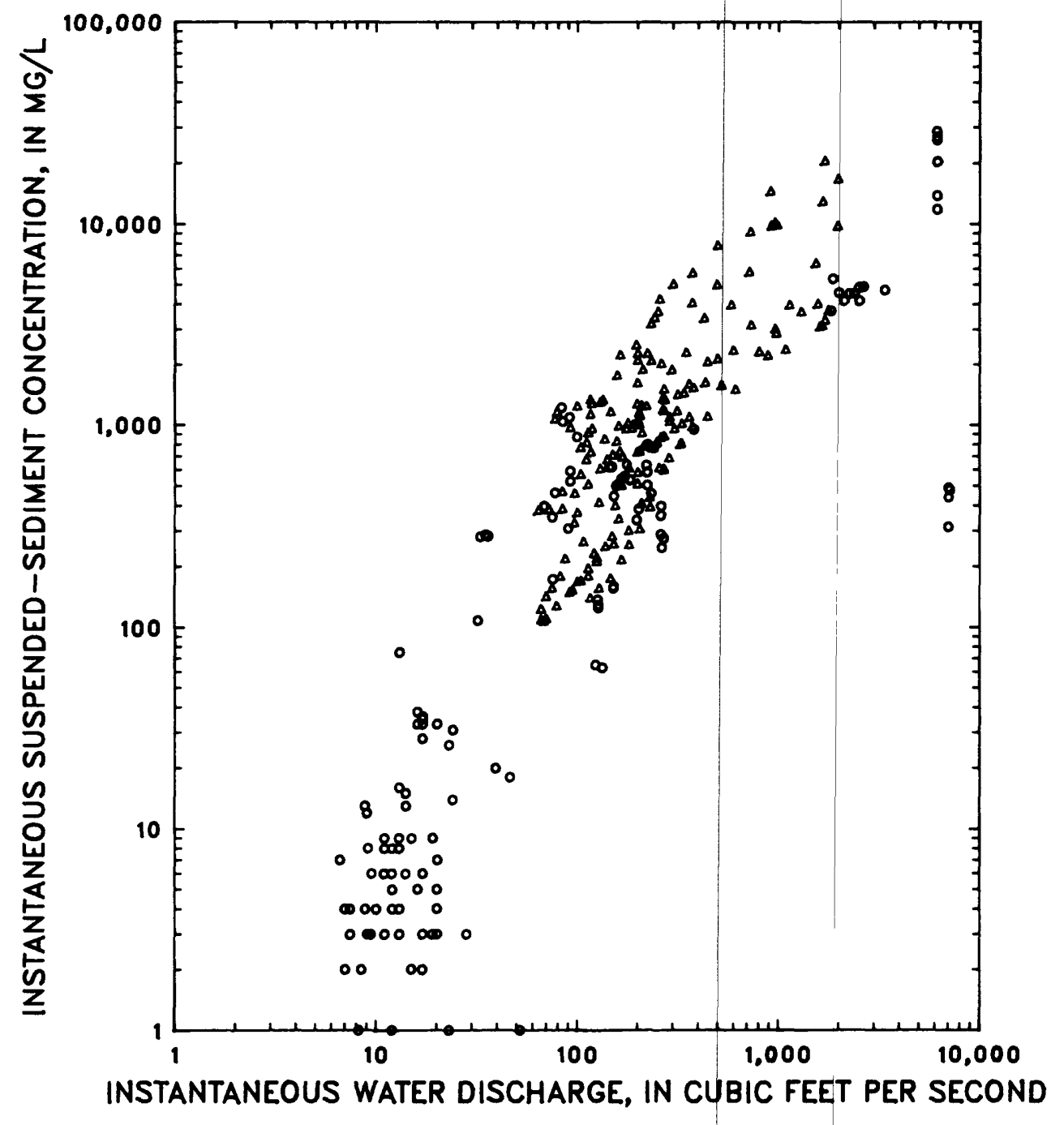

\section{EXPLANATION}

- fIELD SAMPLE

- ISCO SAMPLE

Figure 8.--Reiation of suspended-sediment concentracion to stream discharge for station Rio Turabo at Borinquen. 
Table 9. Suspended-sediment data for samples collected at station Rio Grande de Loiza at Caguas

[US/CM, microsiemens per centimeter; CFS, cubic foot per second; MG/L, milligrams per liter; T/DAY, tons per day; Q $\mathrm{L} / \mathrm{Q}$, ratio of instantaneous water discharge to average water discharge; ..., missing data]

\begin{tabular}{|c|c|c|c|c|c|c|c|c|c|c|c|c|}
\hline DATE & TIME & $\begin{array}{l}\text { SPE- } \\
\text { CIFIC } \\
\text { CON- } \\
\text { DUCT - } \\
\text { ANCE } \\
\text { (US/CM) }\end{array}$ & $\begin{array}{l}\text { STREAM - } \\
\text { FLDW, } \\
\text { INSTAN - } \\
\text { TANEOUS } \\
\text { (CFS) }\end{array}$ & $\begin{array}{l}\text { SEDI - } \\
\text { MENT, } \\
\text { SUS- } \\
\text { PENDED } \\
\text { (MG/L) }\end{array}$ & $\begin{array}{l}\text { SEDI - } \\
\text { MENT, } \\
\text { DIS- } \\
\text { CHARGE, } \\
\text { SUS - } \\
\text { PENDED } \\
\text { (T/DAY) }\end{array}$ & $Q i / Q a$ & OATE & TIME & $\begin{array}{l}\text { SPE- } \\
\text { CIFIC } \\
\text { COH- } \\
\text { DUCT- } \\
\text { ANCE } \\
\text { (US/CM) }\end{array}$ & $\begin{array}{l}\text { STREAM- } \\
\text { FLDW, } \\
\text { INSTAN- } \\
\text { TANEOUS } \\
\text { (CFS) }\end{array}$ & $\begin{array}{l}\text { SEDI - } \\
\text { MENT, } \\
\text { SUS- } \\
\text { PENDED } \\
\text { (MG/L) }\end{array}$ & $\begin{array}{l}\text { SEDI - } \\
\text { MENT, } \\
\text { DIS- } \\
\text { CHARGE, } \\
\text { SUS- } \\
\text { PENDED } \\
\text { (T/DAY) }\end{array}$ \\
\hline
\end{tabular}

WATER YEAR OCTOBER 1983 TO SEPTEMBER 1984

\begin{tabular}{|c|c|c|c|}
\hline $\begin{array}{l}\text { DCT } \\
\text { FEB } \\
\text { FEB } \\
\text { FEB } \\
\text { FEB }\end{array}$ & $\begin{array}{r}4 \\
16 \\
16 \\
16 \\
16\end{array}$ & $\begin{array}{l}1300 \\
1455 \\
1510 \\
152 D \\
1530\end{array}$ & $\begin{array}{c}202 \\
\ldots- \\
\ldots- \\
- \\
-\end{array}$ \\
\hline $\begin{array}{l}\text { FEB } \\
\text { FEB } \\
\text { FEB } \\
\text { FEB } \\
\text { MAR }\end{array}$ & $\begin{array}{l}16 \\
16 \\
16 \\
16 \\
28\end{array}$ & $\begin{array}{l}1535 \\
1544 \\
1554 \\
1604 \\
0845\end{array}$ & $\begin{array}{l}\cdots \\
\cdots \\
\cdots \\
258\end{array}$ \\
\hline
\end{tabular}

APR $\quad 9 \quad 1130$

APR $26 \quad 1000$

MAY $22 \quad 0900$

$\begin{array}{lll}\text { SEPT } & 14 & 1055 \\ \text { SEPT } 14 & 1100\end{array}$

251
266

266
294

$\begin{array}{lr}294 & 32 \\ 128 & 2,650 \\ 117 & 2,600\end{array}$

2,600

SEPT $14 \quad 1105$

SEPT $14 \quad 1110$

$120 \quad 2,570$

$\begin{array}{ll}113 & 2,540 \\ 109 & 2,480\end{array}$

$117 \quad 2,440$

$\begin{array}{lll}\text { SEPT } & 14 & 1120 \\ \text { SEPT } & 14 & 1125\end{array}$

$109 \quad 2,410$

2,360

2,320

2,290

SEPT $14 \quad 1135$

SEPT $14 \quad 1140$

$\begin{array}{lll}\text { SEPT } & 14 & 1145 \\ \text { SEPT } & 14 & 1150\end{array}$

109

109
105

2,220

2,170

\section{307}

307
312

299

51
729
721

721
680

666

293

290

283

289
19

$\begin{array}{ll}647 & 3.7 \\ 632 & 3.6 \\ 607 & 3.5 \\ 611 & 3.5 \\ 2.7 & \end{array}$

SEPT $14 \quad 1155$

SEPT $14 \quad 1200$

SEPT $14 \quad 1215$

$108 \quad 2,130$

$117 \quad 2,090$

$111 \quad 1,940$

$111 \quad 1,730$

$$
\begin{array}{r}
21 \\
134 \\
18 \\
2,310 \\
3,520
\end{array}
$$

$\begin{array}{cc}2.4 & .19 \\ 11 & .14 \\ 16,500 & 12.14 \\ 24,700 & 12\end{array}$

3,700

3,450

2,680

2,610
2,450

3, 300

2,700

$2,680 \quad 16,600$

$2,220 \quad 13,300$

$340 \quad 13,300$

$\begin{array}{ll}2,120 & 12,200 \\ 1,930 & 10,900\end{array}$

$1,820 \quad 9,530$

$2,060 \quad 10,300$

$\begin{array}{rr}1,080 & 5,040\end{array}$

$1,510 \quad 6,690$

1,570

6,690
6,490

$1,190 \quad 4,660$

$\begin{array}{rr}850 & 3,170 \\ 1,040 & 3,680\end{array}$

$1,130 \quad 3,600$

1,180

WATER YEAR OCTOBER 1984 TO SEPTEMBER 1985

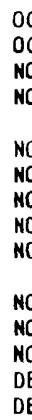

OCT $22 \quad 1624$

$\begin{array}{lrr}\text { OCT } & 22 & 1624 \\ \text { OOV } & 5 & 1405\end{array}$

NOV $5 \quad 1745 \quad \ldots$

$\begin{array}{lll}\text { NOV } & 5 & 1745 \\ \text { NOV } & 5 & 1830\end{array}$

$\begin{array}{lll}\text { NOV } & 5 & 1830 \\ \text { NOV } & 7 & 1110\end{array}$

NOV 71115

$\begin{array}{lll}\text { NOV } & 7 & 1120 \\ \text { NOV } & 7 & 1125\end{array}$

NOV 71130

NOV $14 \quad 0920$

NOV $20 \quad 1635$

$\begin{array}{lll}\mathrm{DEC} & 5 & 1415 \\ \mathrm{DEC} & 7 & 1105\end{array}$
157
175
7,620
7,620
7,520
1,960
1,950
1,940
1,940
1,930
308
228
182
163

48
69
3,500
2,480
3,060
280
284
258
255

258
72
10
58
153

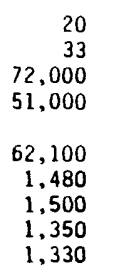

$\quad .70$
34.78
34
34
8.8
8.8
8.7
8.7
8.7
1.4
1.0
.81
.73

8.3
7.7

6.2
5.8

5.3
WATER YEAR OCTOBER 1984 TO SEPTEMBER 1985

$\begin{array}{rrrrr}\text { DEC } & 19 & 1450 & 250 & 13 \\ \text { JAN } & 11 & 1435 & 260 & 10 \\ \text { JAN } & 23 & 1210 & 270 & 7 \\ \text { FEB } & 14 & 1230 & 280 & 5 \\ \text { MAR } & 1 & 1147 & 218 & 10\end{array}$

MAR $11 \quad 1300$

$\begin{array}{lll}\text { MAR } & 25 & 1315 \\ \text { MAR } & 29 & 0830\end{array}$

MAR $29 \quad 0835$

MAR $29 \quad$ D840

256
265

120

$120 \quad 2,110$

$125 \quad 2,030$

MAR $29 \quad 0845$

MAR $29 \quad 0855$

MAR $29 \quad 0905$

$\begin{array}{lll}\text { APR } & 10 & 1047 \\ \text { MAY } & 15 & 1545\end{array}$

$120 \quad 1,990$

1,900

1,820
66

MAY $15 \quad 1555$

$\begin{array}{lll}\text { MAY } & 15 & 1605 \\ \text { MAY } & 15 & 1615\end{array}$

MAY $15 \quad 1625$

MAY $15 \quad 1635$

MAY $15 \quad 1645$

MAY $15 \quad 1655$

MAY $17 \quad 1200$

$\begin{array}{lll}\text { MAY } & 17 & 1205 \\ \text { MAY } & 17 & 1215\end{array}$

MAY $17 \quad 1220$

MAY $17 \quad 1225$

MAY $17 \quad 1230$

MAY $17 \quad 1235$

MAY $17 \quad 1245$

MAY $17 \quad 1250$

MAY $17 \quad 1300$

$\begin{array}{lll}\text { MAY } & 17 & 1316 \\ \text { MAY } & 17 & 1320\end{array}$

MAY $17 \quad 1330$

MAY $17 \quad 1345$

MAY $17 \quad 1400$

MAY $17 \quad 1415$

MAY $17 \quad 1430$

MAY $17 \quad 1445$

$\begin{array}{lll}\text { MAY } 17 & 1500 \\ \text { MAY } 17 & 1502\end{array}$

MAY $17 \quad 1515$

MAY $17 \quad 1545$

MAY $17 \quad 1600$

MAY $17 \quad 1615$

MAY $17 \quad 1630$

MaY $17 \quad 1645$

MAY $17 \quad 1700$

MAY $17 \quad 1715$

MAY $17 \quad 1730$

MAY $17 \quad 1745$

MAY $17 \quad 1800$
11,600

245
76

$73 \quad 11,300$

10,500

9,920

8,670

7,400

16,500

18,200

21,500

22,600

23,700

24,800

23,500

22,300

24,600

23,700

21,800

19,400

18,400

5716,100

$58 \quad 13,700$

5811,600

$\begin{array}{ll}60 & 9,870 \\ 63 & 8,450\end{array}$

637,500

7,500

6,760

6,270

5,260

4,870

4,530

4,280

4,040

3,870

3,680

3,680
3,540

3,420

3,350

3,620
7011,000

$1,700 \quad 8,720$

$1,700 \quad 8,210$

$3,400 \quad 91,100$

$1,780 \quad 16,400$

$\begin{array}{rr}180 & 65 \\ 102 & 30 \\ 76 & 16 \\ 80 & 12 \\ 518 & 142\end{array}$

56
66
2,220

$2,220 \quad 12,500$

$2,100 \quad 11,400$

$1,900 \quad 9,990$

$\begin{array}{rr}55 & 10 \\ 4,100 & 129,000\end{array}$

$4,400 \quad 134,000$

$4,200 \quad 124,000$

$3,300 \quad 93,600$

$3,200 \quad 80,000$

$2,200 \quad 51,500$

$2,800 \quad 56,300$

$12.200 \quad 544,000$

$11,200 \quad 550,000$

$9,940 \quad 577,000$

$10,200 \quad 653,000$

$9,820 \quad 626.000$

$10,200 \quad 683,000$

$9,970 \quad 634,000$

$9,930 \quad 597,000$

$12,300 \quad 817,000$

$8,610 \quad 551,000$

$8,400 \quad 494,000$

$7,400 \quad 388,000$

$6,900 \quad 343,000$

$6,600 \quad 287.000$

$6.300 \quad 233,000$

$5,660 \quad 177,000$

$5,100 \quad 136,000 \quad 44$

$4,700 \quad 107,000 \quad 38$

$4,680 \quad 94,800$

$3,120 \quad 57,000$

$3,440 \quad 62,200$

$3,660 \quad 62,000$

$3,090 \quad 43,900$

$2,410 \quad 31,700$

$2,050 \quad 25,100$

$1,990 \quad 23,000$

$1,820 \quad 19,900$

$1,740 \quad 18,200$

$1,540 \quad 15,300$

$1,370 \quad 13,100$

$\begin{array}{rr}1,090 & 9,860 \\ 1,020 & 9,970\end{array}$
0.60

.49

.34

.45

31

.25

9.2

9.1

8.9

8.1

.29

52

50

49
47

42

39

74

81
96

101

106

111
105

100

110

106

87

82

72

61

33

30
28

23

22

19

18
17

16

16
15
15
16 
Table 9. Suspended-sediment data for samples collected at station Rio Grande de Loiza at Caguas

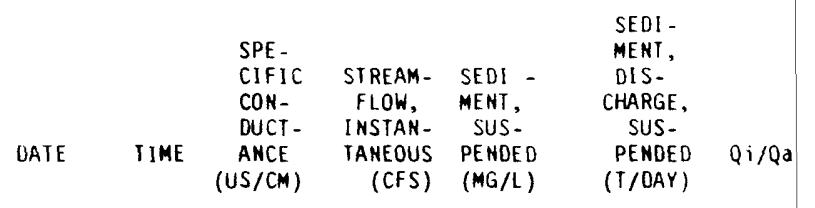

WATER YEAR OCTOBER 1984 TO SEPTEMBER 1985-Cont inued

\begin{tabular}{|c|c|c|c|c|c|c|c|}
\hline $\begin{array}{l}\text { MAY } \\
\text { MAY } \\
\text { MAY } \\
\text { MAY } \\
\text { MAY }\end{array}$ & $\begin{array}{l}17 \\
17 \\
17 \\
17 \\
17\end{array}$ & $\begin{array}{l}1845 \\
1850 \\
1855 \\
1900 \\
1905\end{array}$ & $\begin{array}{r}104 \\
97 \\
96 \\
96 \\
103\end{array}$ & $\begin{array}{l}4,410 \\
4,800 \\
5,190 \\
5,560 \\
5,860\end{array}$ & $\begin{array}{l}1,200 \\
1,370 \\
1,470 \\
1,600 \\
1,830\end{array}$ & $\begin{array}{l}14,300 \\
17,800 \\
20,600 \\
24,100 \\
28,900\end{array}$ & $\begin{array}{l}20 \\
21 \\
23 \\
25 \\
26\end{array}$ \\
\hline $\begin{array}{l}\text { MAY } \\
\text { MAY } \\
\text { MAY } \\
\text { MAY } \\
\text { MAY }\end{array}$ & $\begin{array}{l}17 \\
17 \\
17 \\
17 \\
17\end{array}$ & $\begin{array}{l}1910 \\
1915 \\
1920 \\
1925 \\
1930\end{array}$ & $\begin{array}{l}97 \\
97 \\
8 \mathrm{~B} \\
\mathrm{~B} 8 \\
\mathrm{~B} 8\end{array}$ & $\begin{array}{l}6,130 \\
6,410 \\
6,620 \\
6,840 \\
7,050\end{array}$ & $\begin{array}{l}1,950 \\
2,080 \\
2,440 \\
1,590 \\
2,740\end{array}$ & $\begin{array}{l}32,300 \\
36,000 \\
43,600 \\
29,400 \\
52,200\end{array}$ & $\begin{array}{l}27 \\
29 \\
30 \\
30 \\
31\end{array}$ \\
\hline $\begin{array}{l}\text { MAY } \\
\text { MAY } \\
\text { MAY } \\
\text { MAY } \\
\text { MAY }\end{array}$ & $\begin{array}{l}17 \\
17 \\
17 \\
18 \\
18\end{array}$ & $\begin{array}{l}1945 \\
2000 \\
2030 \\
1000 \\
1030\end{array}$ & $\begin{array}{l}85 \\
84 \\
84 \\
47 \\
44\end{array}$ & $\begin{array}{r}7,380 \\
7,430 \\
7,320 \\
18,600 \\
16,700\end{array}$ & $\begin{array}{l}2,920 \\
3,380 \\
2,220 \\
4,500 \\
3,700\end{array}$ & $\begin{array}{r}58,200 \\
67,800 \\
43,900 \\
226,000 \\
167,000\end{array}$ & $\begin{array}{l}33 \\
33 \\
33 \\
83 \\
75\end{array}$ \\
\hline $\begin{array}{l}\text { MAY } \\
\text { MAY } \\
\text { MAY } \\
\text { MAY } \\
\text { MAY }\end{array}$ & $\begin{array}{l}18 \\
18 \\
18 \\
21 \\
29\end{array}$ & $\begin{array}{l}1200 \\
1230 \\
1232 \\
0825 \\
0800\end{array}$ & $\begin{array}{r}66 \\
55 \\
74 \\
172 \\
194\end{array}$ & $\begin{array}{r}11,300 \\
9,440 \\
9,320 \\
395 \\
181\end{array}$ & $\begin{array}{r}4,300 \\
3,700 \\
4,220 \\
28 \\
151\end{array}$ & $\begin{array}{r}131,000 \\
94,300 \\
106,000 \\
30 \\
74\end{array}$ & $\begin{array}{r}50 \\
42 \\
42 \\
1.8 \\
.81\end{array}$ \\
\hline $\begin{array}{l}\text { JUNE } \\
\text { JUNE } \\
\text { JUNE } \\
\text { JUNE } \\
\text { JULY }\end{array}$ & $\begin{array}{l}7 \\
14 \\
21 \\
28 \\
05\end{array}$ & $\begin{array}{l}0805 \\
0750 \\
0815 \\
0810 \\
0810\end{array}$ & $\begin{array}{l}175 \\
230 \\
225 \\
207 \\
188\end{array}$ & $\begin{array}{r}129 \\
91 \\
79 \\
54 \\
60\end{array}$ & $\begin{array}{l}20 \\
28 \\
45 \\
89 \\
74\end{array}$ & $\begin{array}{l}6.7 \\
6.9 \\
10 \\
13 \\
12\end{array}$ & $\begin{array}{l}.58 \\
.41 \\
.35 \\
.24 \\
.27\end{array}$ \\
\hline $\begin{array}{l}\text { JULY } \\
\text { JULY } \\
\text { JULY } \\
\text { JULY } \\
\text { AUG }\end{array}$ & $\begin{array}{r}16 \\
16 \\
16 \\
25 \\
1\end{array}$ & $\begin{array}{l}0840 \\
0900 \\
0915 \\
0800 \\
0805\end{array}$ & $\begin{array}{l}120 \\
120 \\
130 \\
170 \\
216\end{array}$ & $\begin{array}{r}762 \\
731 \\
710 \\
165 \\
85\end{array}$ & $\begin{array}{r}909 \\
91 B \\
878 \\
99 \\
19\end{array}$ & $\begin{array}{r}1,870 \\
1,810 \\
1,680 \\
44 \\
4.4\end{array}$ & $\begin{array}{l}3.4 \\
3.3 \\
3.2 \\
.74 \\
.38\end{array}$ \\
\hline $\begin{array}{l}\text { AUG } \\
\text { AUG } \\
\text { SEPT } \\
\text { SEPT } \\
\text { SEPT }\end{array}$ & $\begin{array}{r}12 \\
23 \\
6 \\
6 \\
6\end{array}$ & $\begin{array}{l}1325 \\
0830 \\
0810 \\
0820 \\
0835\end{array}$ & $\begin{array}{l}202 \\
200 \\
165 \\
170 \\
165\end{array}$ & $\begin{array}{r}48 \\
52 \\
102 \\
100 \\
97\end{array}$ & $\begin{array}{r}10 \\
58 \\
246 \\
290 \\
278\end{array}$ & $\begin{array}{l}1.3 \\
B .1 \\
68 \\
78 \\
73\end{array}$ & $\begin{array}{l}.21 \\
.23 \\
.46 \\
.45 \\
.43\end{array}$ \\
\hline $\begin{array}{l}\text { SEPT } \\
\text { SEPT } \\
\text { SEPT }\end{array}$ & $\begin{array}{l}24 \\
24 \\
24\end{array}$ & $\begin{array}{l}1740 \\
1750 \\
1755\end{array}$ & $\begin{array}{l}190 \\
200 \\
200\end{array}$ & $\begin{array}{l}507 \\
501 \\
498\end{array}$ & $\begin{array}{l}460 \\
462 \\
440\end{array}$ & $\begin{array}{l}630 \\
625 \\
592\end{array}$ & $\begin{array}{l}2.3 \\
2.3 \\
2.2\end{array}$ \\
\hline
\end{tabular}

WATER YEAR OCTOBER 1985 TO SEPTEMBER 1986

$\begin{array}{lrrrrrr}\text { OCI } & 3 & 1345 & 180 & 443 & 192 & 230 \\ \text { OCT } & 3 & 1350 & 180 & 440 & 192 & 228 \\ \text { OCT } & 6 & 1245 & 50 & 13,300 & 4,520 & 162,000 \\ \text { OCI } & 6 & 1315 & 50 & 12,200 & 5,650 & 186,000 \\ \text { OCI } & 6 & 1345 & 50 & 11,900 & 5,260 & 169,000 \\ & & & & & & \\ \text { OCI } & 6 & 1415 & 60 & 12,600 & 4,220 & 144,000 \\ \text { OCT } & 6 & 1430 & 60 & 12,800 & 3,590 & 124,000 \\ \text { OCT } & 6 & 1445 & 50 & 12,300 & 3,660 & 122,000 \\ \text { OCT } & 6 & 1500 & 60 & 12,100 & 3,530 & 115,000 \\ \text { OCT } & 6 & 1530 & 50 & 11,800 & 3,190 & 102,000 \\ & & & & & & \\ \text { OCT } & 6 & 1600 & 50 & 12,000 & 2,470 & 80,000 \\ \text { OCT } & 6 & 1630 & 50 & 14,900 & 2,800 & 113,000 \\ \text { OCT } & 6 & 1645 & 60 & 15,700 & 6,180 & 262,000 \\ \text { OCT } & 6 & 1700 & 60 & 17,600 & 9,220 & 438,000 \\ \text { OCT } & 6 & 1715 & 50 & 16,900 & 8,680 & 396,000\end{array}$

SEDI -

MENT,

CIFIC STREAM - SEDI - DIS-

CON- FLOW, MENT, CHARGE,

DUCT - INSTAN- SUS- SUSDATE TIME $\begin{gathered}\text { ANCE } \\ \text { (US/CM) }\end{gathered}$
(CFS)

WATER YEAR OCTOBER 1985 TO SEPTEMBER 1986

\begin{tabular}{|c|c|c|c|c|c|c|c|}
\hline $\begin{array}{l}O C T \\
O C T \\
O C T \\
O C T \\
O C T\end{array}$ & $\begin{array}{l}6 \\
6 \\
6 \\
6 \\
7\end{array}$ & $\begin{array}{l}1730 \\
1745 \\
1800 \\
1815 \\
0845\end{array}$ & $\begin{array}{r}50 \\
50 \\
50 \\
50 \\
100\end{array}$ & $\begin{array}{r}16,900 \\
16,000 \\
15,600 \\
15,500 \\
6,800\end{array}$ & $\begin{array}{l}6,840 \\
4,890 \\
4,220 \\
3,580 \\
1,280\end{array}$ & $\begin{array}{r}312,000 \\
211,000 \\
178,000 \\
150,000 \\
23,500\end{array}$ & $\begin{array}{l}75 \\
71 \\
70 \\
70 \\
30\end{array}$ \\
\hline $\begin{array}{l}O C T \\
O C T \\
O C T \\
O C T \\
O C T\end{array}$ & $\begin{array}{l}7 \\
7 \\
7 \\
7 \\
7\end{array}$ & $\begin{array}{l}0915 \\
0945 \\
1000 \\
1045 \\
1115\end{array}$ & $\begin{array}{l}120 \\
100 \\
120 \\
100 \\
100\end{array}$ & $\begin{array}{l}6,590 \\
6,170 \\
6,000 \\
5,810 \\
5,540\end{array}$ & $\begin{array}{r}1,090 \\
973 \\
1,080 \\
952 \\
826\end{array}$ & $\begin{array}{l}19,400 \\
16,200 \\
17,500 \\
14,900 \\
12,400\end{array}$ & $\begin{array}{l}29 \\
28 \\
27 \\
26 \\
25\end{array}$ \\
\hline $\begin{array}{l}O C T \\
O C T \\
O C T \\
O C T \\
O C T\end{array}$ & $\begin{array}{l}7 \\
7 \\
7 \\
7 \\
7\end{array}$ & $\begin{array}{l}1145 \\
1215 \\
1245 \\
1315 \\
1345\end{array}$ & $\begin{array}{l}100 \\
140 \\
130 \\
100 \\
130\end{array}$ & $\begin{array}{l}5,350 \\
5,330 \\
4,910 \\
4,600 \\
4,330\end{array}$ & $\begin{array}{l}822 \\
709 \\
647 \\
616 \\
601\end{array}$ & $\begin{array}{r}11,900 \\
10,200 \\
8,580 \\
7,650 \\
7,030\end{array}$ & $\begin{array}{l}24 \\
24 \\
22 \\
20 \\
19\end{array}$ \\
\hline $\begin{array}{l}O C T \\
O C T \\
O C T \\
O C T \\
O C T\end{array}$ & $\begin{array}{l}7 \\
7 \\
7 \\
7 \\
7\end{array}$ & $\begin{array}{l}1415 \\
1445 \\
1515 \\
1545 \\
1615\end{array}$ & $\begin{array}{l}140 \\
120 \\
130 \\
120 \\
120\end{array}$ & $\begin{array}{l}4,030 \\
3,870 \\
3,760 \\
3,570 \\
3,430\end{array}$ & $\begin{array}{l}562 \\
488 \\
516 \\
424 \\
417\end{array}$ & $\begin{array}{l}6,120 \\
5,100 \\
5,240 \\
4,090 \\
3,860\end{array}$ & $\begin{array}{l}18 \\
17 \\
17 \\
16 \\
15\end{array}$ \\
\hline $\begin{array}{l}\text { OCT } \\
\text { OCT } \\
\text { NOV } \\
\text { NOV } \\
\text { DEC }\end{array}$ & $\begin{array}{r}7 \\
7 \\
14 \\
29 \\
6\end{array}$ & $\begin{array}{l}1715 \\
1815 \\
0800 \\
0810 \\
0910\end{array}$ & $\begin{array}{l}120 \\
120 \\
180 \\
240 \\
250\end{array}$ & $\begin{array}{r}3,520 \\
3,080 \\
288 \\
197 \\
192\end{array}$ & $\begin{array}{l}480 \\
493 \\
185 \\
250 \\
200\end{array}$ & $\begin{array}{r}4,560 \\
4,100 \\
143 \\
133 \\
103\end{array}$ & $\begin{array}{r}16 \\
14 \\
1.3 \\
.88 \\
.86\end{array}$ \\
\hline $\begin{array}{l}\text { DEC } \\
\text { DEC } \\
\text { JAN } \\
\text { JAN } \\
\text { FEB }\end{array}$ & $\begin{array}{l}19 \\
27 \\
16 \\
30 \\
13\end{array}$ & $\begin{array}{l}0930 \\
0805 \\
1255 \\
1240 \\
0855\end{array}$ & $\begin{array}{l}235 \\
235 \\
230 \\
222 \\
250\end{array}$ & $\begin{array}{r}153 \\
118 \\
103 \\
100 \\
83\end{array}$ & $\begin{array}{r}698 \\
247 \\
316 \\
379 \\
51\end{array}$ & $\begin{array}{r}288 \\
78 \\
88 \\
102 \\
11\end{array}$ & $\begin{array}{l}.6 B \\
.53 \\
.46 \\
.45 \\
.37\end{array}$ \\
\hline $\begin{array}{l}\text { FEB } \\
\text { MAR } \\
\text { APR } \\
\text { APR } \\
\text { APR }\end{array}$ & $\begin{array}{r}27 \\
20 \\
7 \\
17 \\
29\end{array}$ & $\begin{array}{l}1020 \\
1145 \\
1235 \\
1200 \\
1615\end{array}$ & $\begin{array}{l}260 \\
280 \\
230 \\
240 \\
100\end{array}$ & $\begin{array}{r}74 \\
46 \\
100 \\
50 \\
3,750\end{array}$ & $\begin{array}{r}43 \\
22 \\
53 \\
49 \\
3,180\end{array}$ & $\begin{array}{r}8.6 \\
2.7 \\
14 \\
6.6 \\
32,200^{6}\end{array}$ & $\begin{array}{r}.33 \\
.20 \\
.45 \\
.22 \\
17\end{array}$ \\
\hline $\begin{array}{l}\text { APR } \\
\text { APR } \\
\text { APR } \\
\text { APR } \\
\text { APR }\end{array}$ & $\begin{array}{l}29 \\
29 \\
29 \\
29 \\
29\end{array}$ & $\begin{array}{l}1630 \\
1645 \\
1700 \\
1715 \\
1730\end{array}$ & $\begin{array}{r}100 \\
100 \\
100 \\
80 \\
80\end{array}$ & $\begin{array}{l}5,400 \\
7,270 \\
8,930 \\
9,370 \\
9,290\end{array}$ & $\begin{array}{l}3,700 \\
5,830 \\
5,960 \\
4,860 \\
3,850\end{array}$ & $\begin{array}{r}54,000 \\
114,000 \\
144,000 \\
123,000 \\
96,600\end{array}$ & $\begin{array}{l}24 \\
32 \\
40 \\
42 \\
41\end{array}$ \\
\hline $\begin{array}{l}\text { APR } \\
\text { APR } \\
\text { APR } \\
\text { APR } \\
\text { APR }\end{array}$ & $\begin{array}{l}29 \\
29 \\
29 \\
29 \\
29\end{array}$ & $\begin{array}{l}1745 \\
1800 \\
1815 \\
1830 \\
1845\end{array}$ & $\begin{array}{l}80 \\
80 \\
80 \\
80 \\
80\end{array}$ & $\begin{array}{l}8,970 \\
8,590 \\
7,830 \\
7,250 \\
6,700\end{array}$ & $\begin{array}{l}3,500 \\
3,020 \\
2,990 \\
2,780 \\
2,630\end{array}$ & $\begin{array}{l}84,800 \\
70,000 \\
63,100 \\
54,400 \\
47,500\end{array}$ & $\begin{array}{l}40 \\
38 \\
35 \\
32 \\
30\end{array}$ \\
\hline $\begin{array}{l}\text { APR } \\
\text { APR } \\
\text { APR } \\
\text { MAY } \\
\text { MAY }\end{array}$ & $\begin{array}{r}29 \\
29 \\
29 \\
1 \\
8\end{array}$ & $\begin{array}{l}1900 \\
1915 \\
1930 \\
0945 \\
0930\end{array}$ & $\begin{array}{r}80 \\
80 \\
90 \\
170 \\
220\end{array}$ & $\begin{array}{r}6,200 \\
5,630 \\
5,200 \\
215 \\
256\end{array}$ & $\begin{array}{r}2,490 \\
2,500 \\
2,400 \\
177 \\
76\end{array}$ & $\begin{array}{r}41,700 \\
38,000 \\
33,700 \\
103 \\
52\end{array}$ & $\begin{array}{l}28 \\
25 \\
23 \\
.96 \\
1.1\end{array}$ \\
\hline $\begin{array}{l}\text { MAY } \\
\text { MAY } \\
\text { MAY } \\
\text { MAY } \\
\text { MAY }\end{array}$ & $\begin{array}{l}8 \\
8 \\
8 \\
8 \\
8\end{array}$ & $\begin{array}{l}1645 \\
1700 \\
1715 \\
1730 \\
1745\end{array}$ & $\begin{array}{r}100 \\
90 \\
80 \\
70 \\
70\end{array}$ & $\begin{array}{r}5,380 \\
10,300 \\
13,900 \\
14,100 \\
13,200\end{array}$ & $\begin{array}{r}885 \\
4,650 \\
6,700 \\
5,880 \\
5,500\end{array}$ & $\begin{array}{r}12,900 \\
129,000 \\
262,000 \\
224,000 \\
196,000\end{array}$ & $\begin{array}{l}24 \\
46 \\
62 \\
63 \\
59\end{array}$ \\
\hline
\end{tabular}


Table 9. Suspended-sediment data for samples collected at station Rio Grande de Loiza at Caguas

\begin{tabular}{|c|c|c|c|c|c|c|c|c|c|c|c|c|}
\hline DATE & IIME & $\begin{array}{l}\text { SPE - } \\
\text { CIFIC } \\
\text { CON- } \\
\text { DUCT- } \\
\text { ANCE }\end{array}$ & $\begin{array}{l}\text { STREAM - } \\
\text { FLOW, } \\
\text { INSTAH- } \\
\text { TANEOUS }\end{array}$ & $\begin{array}{l}\text { SEDI - } \\
\text { MENT, } \\
\text { SUS- } \\
\text { PENDED }\end{array}$ & $\begin{array}{l}\text { SEDI - } \\
\text { MENT, } \\
\text { DIS- } \\
\text { CHARGE, } \\
\text { SUS- } \\
\text { PENDED }\end{array}$ & Qi/Qa & DATE & TIME & $\begin{array}{l}\text { SPL - } \\
\text { CIFIC } \\
\text { CON- } \\
\text { OUCT- } \\
\text { ANCE }\end{array}$ & $\begin{array}{l}\text { STREAK- } \\
\text { FLOW, } \\
\text { INSTAN- } \\
\text { TANEOUS }\end{array}$ & $\begin{array}{l}\text { SEOI - } \\
\text { MENT, } \\
\text { SUS- } \\
\text { PENDED }\end{array}$ & $\begin{array}{l}\text { SEDI- } \\
\text { MENT, } \\
\text { DIS- } \\
\text { CHARGE, } \\
\text { SUS- } \\
\text { PENDED }\end{array}$ \\
\hline & & (US/CM) & (CFS) & $(M G / L)$ & (T/DAY) & & & & (US/CM) & (CFS) & $(M G / L)$ & (T/DAY) \\
\hline
\end{tabular}

WATER YEAR DCTOBER 1985 TO SEPIEMBER 1986 -Cont inued

\begin{tabular}{|c|c|c|c|c|c|c|c|c|c|c|c|c|c|c|c|}
\hline $\begin{array}{l}\text { MAY } \\
\text { MAY } \\
\text { MAY } \\
\text { MAY } \\
\text { MAY }\end{array}$ & $\begin{array}{l}8 \\
8 \\
8 \\
8 \\
8\end{array}$ & $\begin{array}{l}1800 \\
1815 \\
1830 \\
1845 \\
1900\end{array}$ & $\begin{array}{l}60 \\
60 \\
60 \\
80 \\
80\end{array}$ & $\begin{array}{r}12,400 \\
11,500 \\
9,620 \\
7,970 \\
6,930\end{array}$ & $\begin{array}{l}4,930 \\
4,290 \\
3,540 \\
3,480 \\
3,690\end{array}$ & $\begin{array}{r}165,000 \\
133,000 \\
92,000 \\
74,900 \\
69,000\end{array}$ & $\begin{array}{l}55 \\
51 \\
43 \\
36 \\
31\end{array}$ & $\begin{array}{l}\text { MAY } \\
\text { MAY } \\
\text { MAY } \\
\text { MAY } \\
\text { MAY }\end{array}$ & $\begin{array}{l}18 \\
18 \\
18 \\
18 \\
18\end{array}$ & $\begin{array}{l}1330 \\
1345 \\
1400 \\
1415 \\
1430\end{array}$ & $\begin{array}{l}80 \\
70 \\
60 \\
50 \\
50\end{array}$ & $\begin{array}{l}8,480 \\
7,920 \\
7,360 \\
6,800 \\
6,240\end{array}$ & $\begin{array}{l}1,720 \\
1,870 \\
2,020 \\
1,910 \\
1,890\end{array}$ & $\begin{array}{l}39,300 \\
40,000 \\
40,200 \\
35,100 \\
31,900\end{array}$ & $\begin{array}{l}38 \\
35 \\
33 \\
30 \\
28\end{array}$ \\
\hline $\begin{array}{l}\text { MAY } \\
\text { MAY } \\
\text { MAY } \\
\text { MAY } \\
\text { MAY }\end{array}$ & $\begin{array}{r}8 \\
8 \\
8 \\
8 \\
13\end{array}$ & $\begin{array}{l}1915 \\
1930 \\
1945 \\
2000 \\
0815\end{array}$ & $\begin{array}{r}80 \\
60 \\
70 \\
70 \\
110\end{array}$ & $\begin{array}{l}6,020 \\
5,820 \\
5,580 \\
5,170 \\
4,450\end{array}$ & $\begin{array}{l}3,700 \\
3,650 \\
3,810 \\
3,640 \\
1,411\end{array}$ & $\begin{array}{l}60,200 \\
57,400 \\
57,400 \\
50,800 \\
17,000\end{array}$ & $\begin{array}{l}27 \\
26 \\
25 \\
23 \\
20\end{array}$ & $\begin{array}{l}\text { MAY } \\
\text { MAY } \\
\text { MAY } \\
\text { MAY } \\
\text { MAY }\end{array}$ & $\begin{array}{l}18 \\
18 \\
18 \\
18 \\
19\end{array}$ & $\begin{array}{l}1445 \\
1500 \\
1515 \\
1530 \\
1130\end{array}$ & $\begin{array}{r}40 \\
40 \\
40 \\
40 \\
160\end{array}$ & $\begin{array}{r}5,680 \\
5,120 \\
4,710 \\
4,300 \\
482\end{array}$ & $\begin{array}{r}1,850 \\
1,870 \\
1,520 \\
1,510 \\
83\end{array}$ & $\begin{array}{r}28,400 \\
25,800 \\
19,300 \\
17,500 \\
108\end{array}$ & $\begin{array}{r}25 \\
23 \\
21 \\
19 \\
2.2\end{array}$ \\
\hline $\begin{array}{l}\text { MAY } \\
\text { MAY } \\
\text { MAY } \\
\text { MAY } \\
\text { MAY }\end{array}$ & $\begin{array}{l}13 \\
13 \\
13 \\
13 \\
13\end{array}$ & $\begin{array}{l}0830 \\
0845 \\
0900 \\
0915 \\
0930\end{array}$ & $\begin{array}{r}110 \\
110 \\
110 \\
100 \\
90\end{array}$ & $\begin{array}{r}7,600 \\
10,600 \\
13,700 \\
15,200 \\
16,700\end{array}$ & $\begin{array}{l}2,040 \\
2,600 \\
4,460 \\
5,190 \\
4,740\end{array}$ & $\begin{array}{r}42,000 \\
74,400 \\
165,000 \\
213,000 \\
214,000\end{array}$ & $\begin{array}{l}34 \\
47 \\
61 \\
68 \\
75\end{array}$ & $\begin{array}{l}\text { MAY } \\
\text { MAY } \\
\text { MAY } \\
\text { MAY } \\
\text { MAY }\end{array}$ & $\begin{array}{l}31 \\
31 \\
31 \\
31 \\
31\end{array}$ & $\begin{array}{l}0615 \\
0630 \\
0645 \\
0700 \\
0715\end{array}$ & $\begin{array}{l}160 \\
140 \\
140 \\
120 \\
100\end{array}$ & $\begin{array}{l}4,450 \\
6,440 \\
5,780 \\
5,110 \\
4,800\end{array}$ & $\begin{array}{r}982 \\
1,760 \\
4,910 \\
2,270 \\
1,980\end{array}$ & $\begin{array}{l}11,800 \\
30,700 \\
76,600 \\
31,300 \\
25,600\end{array}$ & $\begin{array}{l}20 \\
29 \\
26 \\
23 \\
21\end{array}$ \\
\hline $\begin{array}{l}\text { MAY } \\
\text { MAY } \\
\text { MAY } \\
\text { MAY } \\
\text { MAY }\end{array}$ & $\begin{array}{l}13 \\
13 \\
13 \\
13 \\
13\end{array}$ & $\begin{array}{l}0945 \\
1000 \\
1015 \\
1030 \\
1045\end{array}$ & $\begin{array}{r}90 \\
90 \\
90 \\
100 \\
100\end{array}$ & $\begin{array}{l}16,300 \\
15,900 \\
14,400 \\
12,900 \\
11,400\end{array}$ & $\begin{array}{l}5,550 \\
5,720 \\
4,620 \\
3,440 \\
2,740\end{array}$ & $\begin{array}{r}244,000 \\
246,000 \\
180,000 \\
120,000 \\
84,200\end{array}$ & $\begin{array}{l}73 \\
71 \\
64 \\
58 \\
51\end{array}$ & $\begin{array}{l}\text { MAY } \\
\text { MAY } \\
\text { JUNE } \\
\text { JUNE } \\
\text { JULY }\end{array}$ & $\begin{array}{r}31 \\
31 \\
5 \\
26 \\
7\end{array}$ & $\begin{array}{l}0730 \\
0745 \\
0830 \\
1030 \\
1300\end{array}$ & $\begin{array}{l}100 \\
100 \\
240 \\
250 \\
210\end{array}$ & $\begin{array}{r}4,480 \\
4,170 \\
132 \\
103 \\
129\end{array}$ & $\begin{array}{r}1,520 \\
1,520 \\
41 \\
18 \\
38\end{array}$ & $\begin{array}{r}18,400 \\
17,100 \\
15 \\
5.0 \\
13\end{array}$ & $\begin{array}{l}20 \\
19 \\
.59 \\
.46 \\
.58\end{array}$ \\
\hline $\begin{array}{l}\text { MAY } \\
\text { MAY } \\
\text { MAY }\end{array}$ & $\begin{array}{l}13 \\
13 \\
13\end{array}$ & $\begin{array}{l}1100 \\
1115 \\
1130\end{array}$ & $\begin{array}{l}100 \\
100 \\
100\end{array}$ & $\begin{array}{l}9,960 \\
7,850 \\
6,790\end{array}$ & $\begin{array}{l}2,930 \\
3,200 \\
3,720\end{array}$ & $\begin{array}{l}78,800 \\
67,900 \\
68,200\end{array}$ & $\begin{array}{l}44 \\
35 \\
30\end{array}$ & $\begin{array}{l}\text { AUG } \\
\text { SEPT } \\
\text { SEPT }\end{array}$ & $\begin{array}{r}12 \\
2 \\
15\end{array}$ & $\begin{array}{l}1310 \\
0930 \\
0845\end{array}$ & $\begin{array}{l}220 \\
230 \\
220\end{array}$ & $\begin{array}{r}115 \\
222 \\
52\end{array}$ & $\begin{array}{l}630 \\
136 \\
197\end{array}$ & $\begin{array}{r}196 \\
81 \\
28\end{array}$ & $\begin{array}{l}.51 \\
.99 \\
.23\end{array}$ \\
\hline
\end{tabular}

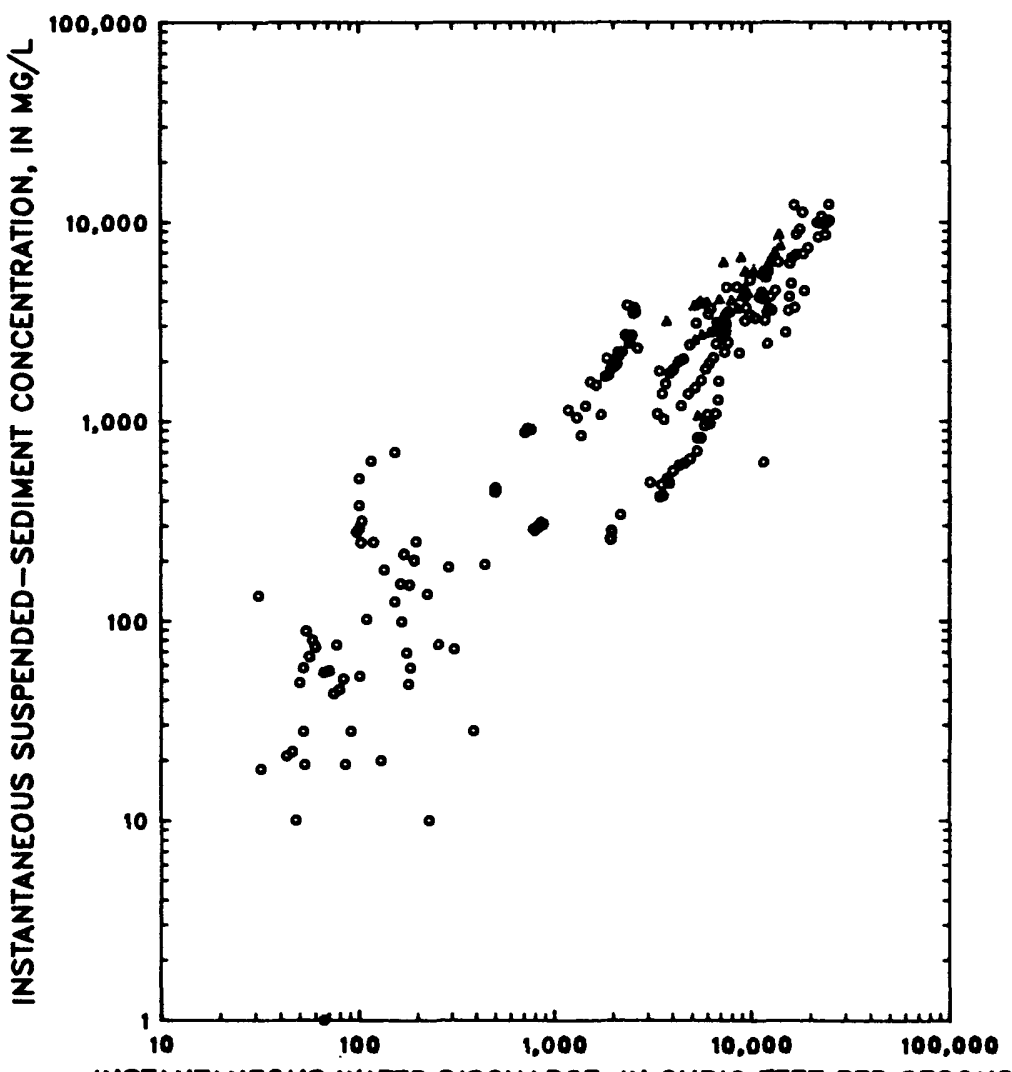

EXPLANATION

- field SAMPLe

- ISCO SAMPLE

INSTANTANEOUS WATER DISCHARGE, IN CUBIC FEET PER SECOND

Figure 9.--Relation of suspended-sediment concentration to stream discharge for station Rio Grande de Loiza at Caguas. 
Table 10. Suspended-sediment data for samples collected at station Quebrada Caimito near Juncos

[US/CM, microsiemens per centimeter; CFS, Cubic foot per second; MG/L, milligrams per liter; T/DAY, tons per day; Qi/Qa, ratio of instantaneous water discharge to average water discharge; .... missing data]

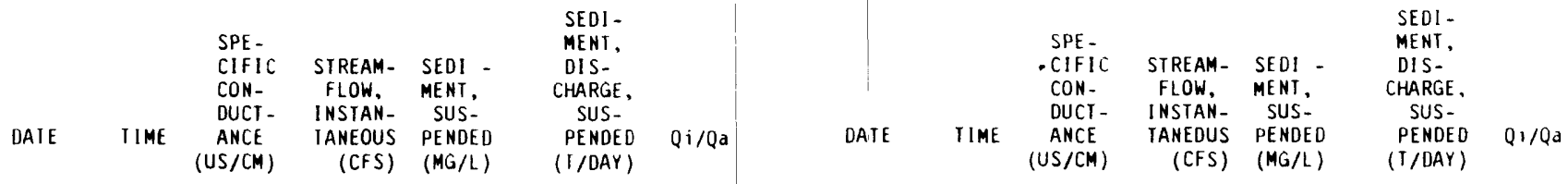

WAIER YEAR OCTOBER 1983 TD SEPIEMBER 1984

\begin{tabular}{lrrrrrrr|} 
IEB & 21 & 1017 & 369 & .12 & 27 & 0.01 & 0.09 \\
MAY & 30 & 1245 & 346 & .18 & 5 & 0 & .14 \\
JUNE & 12 & 0925 & 340 & .33 & 2 & 0 & .25 \\
JULY & 5 & 1045 & 254 & 2.3 & 15 & .09 & 1.8 \\
AUG & 28 & 1055 & 307 & .56 & 65 & .10 & .43 \\
& & & & & & & \\
SEPT 14 & 1145 & 278 & 1.5 & 32 & .13 & 1.1 \\
SEPI 14 & 1200 & 278 & 1.5 & 37 & .15 & 1.1 \\
SEPI 14 & 1230 & 278 & 1.5 & 43 & .17 & 1.1 \\
SEPT 19 & 1215 & 248 & 2.5 & 40 & .27 & 1.9 \\
SEPT 19 & 1300 & 243 & 2.5 & 36 & .27 & 1.9 \\
& & & & & & .87 & 1.9
\end{tabular}

WAIER YEAR OCIOBER 1984 TO SEPIEMBEP 1985

\begin{tabular}{|c|c|c|c|c|c|c|c|}
\hline $\begin{array}{l}\text { OCT } \\
\text { OCI }\end{array}$ & $\begin{array}{l}25 \\
29\end{array}$ & $\begin{array}{l}1315 \\
1245\end{array}$ & 333 & $\begin{array}{r}.71 \\
50\end{array}$ & $?$ & $\begin{array}{l}0 \\
0\end{array}$ & $\begin{array}{r}.54 \\
38\end{array}$ \\
\hline NOV & 5 & $\begin{array}{l}1245 \\
1535\end{array}$ & 80 & $122^{.50}$ & 1.670 & 550 & $94^{\circ 00}$ \\
\hline NOV & 5 & 1555 & 80 & 116 & 2470 & 774 & 89 \\
\hline NOV & 7 & 1130 & 159 & 35 & 149 & 14 & 27 \\
\hline NOV & 7 & 1215 & 165 & 29 & 101 & 7.9 & 22 \\
\hline NOV & 7 & 1245 & 166 & 28 & 139 & 11 & 22 \\
\hline NOV & 7 & 1315 & 163 & 29 & 127 & 10 & 22 \\
\hline NOV & 7 & 1320 & 163 & 35 & 168 & 16 & 27 \\
\hline NOV & 7 & 1325 & 160 & 41 & 245 & 27 & 32 \\
\hline NOV & 7 & 1330 & 156 & 46 & 421 & 52 & 35 \\
\hline NOV & 7 & 1335 & 150 & 49 & 518 & 69 & 38 \\
\hline NOV & 14 & 1330 & 323 & 1.4 & 33 & .12 & 1.1 \\
\hline NOV & 20 & 1445 & 345 & .88 & 7 & .02 & .68 \\
\hline DEC & 5 & 1230 & 380 & .39 & $\ddot{z}$ & $u$ & .30 \\
\hline UEC & 19 & 1308 & 374 & .27 & 4 & .01 & .21 \\
\hline JAN & 11 & 1250 & 385 & .24 & 53 & .07 & .18 \\
\hline JAN & 21 & 1130 & 368 & .16 & 45 & .04 & .12 \\
\hline FEB & 6 & 1200 & 423 & .05 & 5 & $u$ & .04 \\
\hline MAR & 7 & 1330 & 392 & .14 & 2 & 0 & .10 \\
\hline MAY & 16 & 1300 & 190 & 11 & 26 & .77 & 8.5 \\
\hline MAY & 17 & 1300 & 124 & 38 & 373 & 35 & 29 \\
\hline MAY & 17 & 1305 & 125 & 36 & 342 & 33 & 28 \\
\hline MAY & 20 & 1400 & 243 & 4.2 & 12 & .14 & 3.2 \\
\hline MAY & 20 & 1720 & 248 & 3.5 & 12 & .11 & 2.7 \\
\hline MAY & 28 & 1410 & 264 & اذذ. & 1 & 0 & .24 \\
\hline JUNE & 5 & 1145 & 396 & .18 & 3 & 0 & .14 \\
\hline JULY & 15 & 1415 & 400 & .50 & 2 & .01 & .38 \\
\hline JULY & 16 & 1005 & 235 & 5.4 & 157 & 2.3 & 4.1 \\
\hline JULY & 16 & 1015 & 235 & 4.2 & 145 & 1.6 & 3.2 \\
\hline JULY & 16 & 1045 & 245 & 4.2 & 78 & .88 & 3.2 \\
\hline JULY & 22 & 1310 & 308 & .31 & 1 & 0 & .24 \\
\hline SEPT & 13 & 1510 & 150 & 25 & $\angle 16$ & 15 & 19 \\
\hline SEPT & 30 & 1205 & 330 & .71 & 17 & .03 & .55 \\
\hline
\end{tabular}

WAIER YEAR OCTOBER 1985 TO SEPIEMBER 1986

\begin{tabular}{|c|c|c|c|c|c|c|c|}
\hline $\mathrm{OCT}$ & 3 & 0927 & 220 & 5.0 & 36 & 0.49 & 3.8 \\
\hline OCT & 3 & 0928 & 220 & 5.0 & 40 & .54 & 3.8 \\
\hline $\mathrm{OCI}$ & 3 & 0929 & 220 & 5.0 & 49 & .66 & 3.8 \\
\hline $\mathrm{OCT}$ & 3 & 1025 & 220 & 5.0 & 24 & .32 & 3.8 \\
\hline DCT & 7 & 0830 & 130 & 91 & 750 & $1 \mathrm{~B} 4$ & 70 \\
\hline $\mathrm{OCT}$ & 7 & 0840 & 130 & 83 & 325 & 73 & 64 \\
\hline $\mathrm{OCT}$ & 7 & 0850 & 130 & 77 & 622 & 129 & 59 \\
\hline OCI & 7 & 0900 & 140 & 74 & 458 & 92 & 57 \\
\hline $\mathrm{DCI}$ & 7 & 0915 & 160 & 71 & +49 & 86 & 55 \\
\hline $\mathrm{OCI}$ & 7 & 0930 & 150 & 66 & 292 & 52 & 51 \\
\hline $0 \mathrm{CT}$ & 7 & 0945 & 160 & 61 & 266 & 44 & 47 \\
\hline $\mathrm{OCT}$ & 7 & 1000 & 170 & 60 & 346 & 56 & 46 \\
\hline$O C T$ & 7 & 1030 & 160 & 59 & 282 & 45 & 45 \\
\hline$O C T$ & 7 & 1100 & 160 & 52 & 249 & 35 & 40 \\
\hline $0 \mathrm{CI}$ & 7 & 1430 & 170 & 43 & 137 & 16 & 33 \\
\hline OCT & 24 & 1000 & 190 & 6.1 & 73 & 1.2 & 4.7 \\
\hline $0 \mathrm{CI}$ & 24 & 1010 & 170 & B. 6 & 482 & 11 & 6.6 \\
\hline $\mathrm{OCI}$ & 24 & 1020 & 160 & 14 & 530 & 20 & 11 \\
\hline $\mathrm{OCT}$ & 24 & 1025 & 140 & 18 & 786 & 38 & 14 \\
\hline $\mathrm{OCI}$ & 24 & 1030 & 140 & 22 & 988 & 59 & 17 \\
\hline $\mathrm{OCT}$ & 24 & 1035 & 160 & $2 B$ & 1,130 & 85 & 22 \\
\hline $\mathrm{OCT}$ & 24 & 1036 & 130 & 29 & 1,530 & 120 & 22 \\
\hline $\mathrm{OCI}$ & 24 & 1040 & 100 & 34 & 1,420 & 130 & 26 \\
\hline $\mathrm{OCT}$ & 24 & 1050 & 120 & 40 & 966 & 104 & 31 \\
\hline OCT & 24 & 1100 & 110 & 34 & 771 & 71 & 26 \\
\hline $\mathrm{OCT}$ & 24 & 1115 & 130 & 29 & 1,980 & 155 & 22 \\
\hline $\mathrm{OCT}$ & 24 & 1145 & 140 & 18 & 1,040 & 50 & 14 \\
\hline $\mathrm{OCI}$ & 24 & 1215 & 150 & 13 & 404 & 14 & 10 \\
\hline $\mathrm{OCT}$ & 29 & 1008 & 170 & 7.7 & 146 & 3.0 & 5.9 \\
\hline NOV & 12 & 1300 & 140 & 2.7 & 166 & 1.2 & 2.1 \\
\hline NOV & 18 & 1325 & 240 & 3.1 & 45 & .38 & 2.4 \\
\hline DEC & 2 & 1000 & 360 & .51 & 13 & .02 & .40 \\
\hline DEC & 9 & 1325 & 360 & .26 & 6 & 0. & .20 \\
\hline DEC & 16 & 1410 & 370 & .26 & 8 & .01 & .20 \\
\hline$D E C$ & 23 & 1210 & 375 & .26 & 5 & 0 & .20 \\
\hline JAN & 16 & 0905 & 375 & .26 & $\iota$ & 0. & .20 \\
\hline JAN & 23 & 1015 & 370 & .32 & 2 & 0. & .25 \\
\hline FEB & 3 & 1240 & 360 & .26 & 11 & .01 & .20 \\
\hline FEB & 10 & 1245 & 365 & .26 & 36 & .02 & .20 \\
\hline MAY & 14 & 1325 & 185 & 14 & 48 & 1.8 & 11 \\
\hline JUNE & 2 & 1015 & 290 & 2.2 & 16 & .10 & 1.7 \\
\hline JUNE & 5 & 1000 & 340 & .40 & 349 & .38 & .31 \\
\hline JUNE & 9 & 1010 & 340 & 2.6 & 332 & 2.3 & 2 \\
\hline JUNE & 9 & 1110 & 275 & 2.0 & 144 & .78 & 1.5 \\
\hline AUG & 29 & 1220 & 180 & 14 & 75 & 2.8 & 11 \\
\hline
\end{tabular}




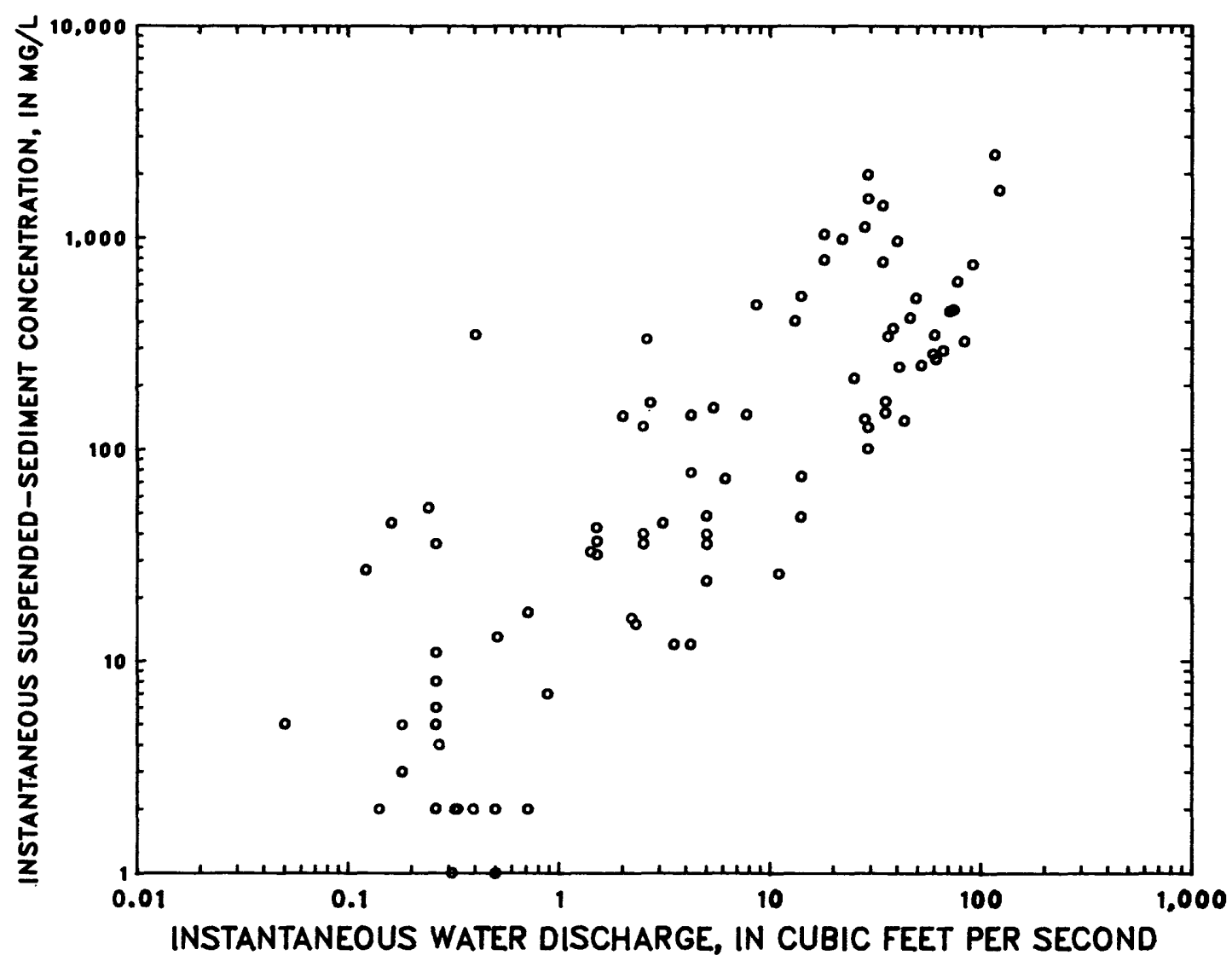

Figure 10.--Relation of suspended-sediment concentration to stream discharge for station Quebrada Caimito near Juncos. 
Táble 11. Suspended-sediment data for samples collected at station Rio Valenciano near Juncos

[US/CM, microsiemens per cent imeter; CFS, cubic foot per second; MG/L, milligrams per Iiter; T/DAY, tons per day; Qi/Qa, ratio of instantaneous water discharge to average water discharge; .... missing data]

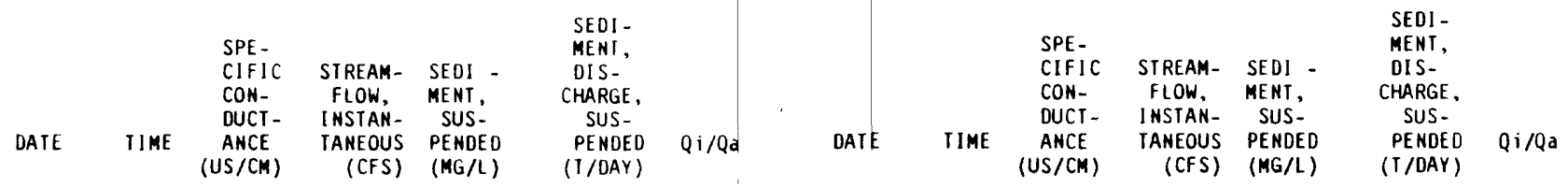

WATER YEAR DCTOBER 1983 TO SEPTEMBER 19B4

\begin{tabular}{|c|c|c|c|c|c|c|c|}
\hline $\begin{array}{l}\text { NOV } \\
\text { FEB } \\
\text { MAR } \\
\text { MAR } \\
\text { APR }\end{array}$ & $\begin{array}{l}22 \\
22 \\
13 \\
28 \\
10\end{array}$ & $\begin{array}{l}1455 \\
1005 \\
1215 \\
1145 \\
0830\end{array}$ & $\begin{array}{l}242 \\
280 \\
274 \\
276 \\
279\end{array}$ & $\begin{array}{l}18 \\
16 \\
11 \\
9 \\
6.8\end{array}$ & $\begin{array}{r}2 \\
33 \\
0 \\
5 \\
3\end{array}$ & $\begin{array}{l}0.10 \\
1.4 \\
0 \\
.12 \\
.06\end{array}$ & $\begin{array}{r}1.1 \\
1.0 \\
1.0 \\
1.0 \\
.94\end{array}$ \\
\hline $\begin{array}{l}\text { APR } \\
\text { MAY } \\
\text { SEPT } \\
\text { SEPT } \\
\text { SEPT }\end{array}$ & $\begin{array}{l}25 \\
16 \\
14 \\
14 \\
14\end{array}$ & $\begin{array}{l}1000 \\
1230 \\
1145 \\
1205 \\
1220\end{array}$ & $\begin{array}{r}262 \\
294 \\
84 \\
98 \\
90\end{array}$ & $\begin{array}{c}\quad 6 \\
\text { B. } 3 \\
846 \\
743 \\
682\end{array}$ & $\begin{array}{r}1 \\
25 \\
700 \\
747 \\
704\end{array}$ & $\begin{array}{l}1,600^{.02} \\
1,500 \\
1,300\end{array}$ & $\begin{array}{l}1.0 \\
1.2 \\
1.2 \\
1.1 \\
.99\end{array}$ \\
\hline $\begin{array}{l}\text { SEPT } \\
\text { SEPT } \\
\text { SEPT }\end{array}$ & $\begin{array}{l}14 \\
14 \\
14\end{array}$ & $\begin{array}{l}1235 \\
1248 \\
1300\end{array}$ & $\begin{array}{l}100 \\
108 \\
100\end{array}$ & $\begin{array}{l}627 \\
574 \\
545\end{array}$ & $\begin{array}{l}686 \\
631 \\
496\end{array}$ & $\begin{array}{r}1,160 \\
978 \\
730\end{array}$ & $\begin{array}{l}.91 \\
.83 \\
.79\end{array}$ \\
\hline
\end{tabular}

WATER YEAR OCTOBER 1984 TO SEPTEMBER 1985

\begin{tabular}{|c|c|c|c|c|}
\hline$O C T$ & 25 & 1230 & 252 & 71 \\
\hline $0 C 1$ & 25 & 1300 & 247 & 67 \\
\hline $\mathrm{OCT}$ & 26 & 1145 & 222 & 48 \\
\hline OCT & 29 & 1200 & & 35 \\
\hline $\mathrm{OCT}$ & 31 & 1050 & 228 & 30 \\
\hline NOV & 5 & 1525 & 79 & 265 \\
\hline NOV & 8 & 1655 & 183 & 123 \\
\hline NOV & 9 & 1312 & 210 & 117 \\
\hline NOV & 13 & 1435 & 212 & 68 \\
\hline MOV & 14 & 1224 & 220 & 64 \\
\hline NOV & 15 & 0900 & 195 & 80 \\
\hline NOV & 20 & 1415 & 232 & 41 \\
\hline NOV & 23 & 1020 & 226 & 64 \\
\hline NOV & 26 & 1305 & 212 & 36 \\
\hline NOV & 28 & 1115 & 232 & 33 \\
\hline HOV & 30 & 1115 & 250 & 32 \\
\hline DEC & 3 & 1035 & 240 & 50 \\
\hline DEC & 5 & 1155 & 260 & 29 \\
\hline DEC & 10 & 1107 & 255 & 26 \\
\hline DEC & 12 & 1123 & 200 & 55 \\
\hline DEC & 13 & 1130 & 190 & 54 \\
\hline DEC & 17 & 1340 & 240 & 35 \\
\hline DEC & 19 & 1207 & 240 & 25 \\
\hline$D E C$ & 20 & 1328 & 245 & 23 \\
\hline $\mathrm{DEC}$ & 26 & 1000 & 240 & 28 \\
\hline $\mathrm{DEC}$ & 27 & 1050 & 265 & 25 \\
\hline JAN & 8 & 1130 & 274 & 21 \\
\hline JAN & 10 & 1212 & 270 & 20 \\
\hline JAN & 15 & 1230 & 303 & 18 \\
\hline JAN & 17 & 1120 & 267 & 17 \\
\hline JAN & 18 & 1030 & 272 & 17 \\
\hline JAN & 21 & 1100 & 270 & 16 \\
\hline JAN & 25 & 0930 & 280 & 15 \\
\hline JAN & 28 & 1035 & 292 & 16 \\
\hline JAN & 30 & 1145 & 293 & 17 \\
\hline FEB & 1 & 1300 & 286 & 13 \\
\hline FEB & 4 & 1200 & 286 & 13 \\
\hline FEB & 6 & 1130 & 278 & 15 \\
\hline FEB & 8 & 1220 & 296 & 11 \\
\hline FEB & 14 & 1031 & 280 & 10 \\
\hline FEB & 19 & 1100 & 305 & 22 \\
\hline FEB & 22 & 1115 & $2 \mathrm{~B} 2$ & 12 \\
\hline FEB & 26 & 1120 & 272 & 12 \\
\hline MAR & 1 & $104 B$ & 284 & 17 \\
\hline MAR & 5 & 1221 & 307 & 10 \\
\hline
\end{tabular}

$4.1 \quad 1.0$

$3.4 \quad 1.1$

$\begin{array}{rr}13 & .91 \\ 5.5 & 1.2 \\ 3.8 & 1.0 \\ 2.2 & .45\end{array}$

$\begin{array}{ll}3.9 & .55\end{array}$

$4.2 \quad .49$

$\begin{array}{ll}2.4 & .41 \\ 2.9 & .39\end{array}$

$\begin{array}{ll}4.8 & .35\end{array}$

$3.1 \quad .33$

$\begin{array}{ll}2.2 & .33 \\ 2.1 & .31\end{array}$

$2.0 \quad .29$

$\begin{array}{ll}.04 & .31 \\ .04 & .33\end{array}$

$2.0 \quad .33$

$\begin{array}{ll}2.1 & .33 \\ & .19\end{array}$
WATER YEAR OCTOBER 1984 TO SEPTEMBER 1985

\begin{tabular}{l|rrrr} 
MAR & 6 & 1300 & 337 & 14 \\
MAR & 7 & 1240 & 307 & 4 \\
MAR & 11 & 1030 & 319 & 1 \\
MAR & 13 & 1145 & 310 & 1 \\
MAR & 15 & 1000 & 310 & 1 \\
& & & & \\
MAR & 19 & 1015 & 290 & 1 \\
MAR & 20 & 0945 & 310 & 1 \\
MAR & 21 & 1235 & 310 & 1 \\
MAR & 25 & 1215 & 310 & \\
MAR & 27 & 1102 & 255 &
\end{tabular}

14
45
13
12
11
12
11
11
9.7
9.4

14
77
10
26
4
16
5
5
4
12

$0.53 \quad 0.27$ $\begin{array}{ll}9.4 & .88\end{array}$ $\begin{array}{ll}.35 & .25 \\ .84 & .23\end{array}$ $\begin{array}{ll}.12 & .21\end{array}$

\begin{tabular}{l|ll} 
MAR & 29 & 1234
\end{tabular}

\begin{tabular}{l|ll} 
MAR & 29 & 1239
\end{tabular}

APR 11105

APR B 114

150

150
160
270

160
156

$\begin{array}{llll}\text { APR } & 26 \quad 1420 \quad 200\end{array}$

MAY $15 \quad 0930 \quad 122$

122
133
133

MAY $16 \quad 1000$

MAY $17 \quad 1435$

MAY $17 \quad 1450$

\begin{tabular}{l|ll} 
MAY 18 & 1630
\end{tabular}

MAY $20 \quad 1640$

$\begin{array}{llll}\text { MAY } & 28 & 1320 & 189\end{array}$

\begin{tabular}{l|rr} 
MAY & 29 & 0920 \\
JUNE & 5 & 1115
\end{tabular}

JUNE $7 \quad 0918$

\begin{tabular}{l|rr} 
JUNE & 7 & 0918 \\
JUNE & 10 & 1340
\end{tabular}

JUNE $14 \quad 0905$

235

JUNE $21 \quad 0900 \quad 240$

$\begin{array}{ll}.52 & .23 \\ .15 & .21 \\ .15 & .21 \\ .10 & .19 \\ .30 & .18\end{array}$

$.30 \quad .18$

\begin{tabular}{l|lll} 
JUNE & 28 & 1200 & 217
\end{tabular}

217
210

JULY 11325

JUIY $5 \quad 1215$

JUIY $8 \quad 1250$

210
208

207
225

$38 \mathrm{~B}$

$38 \mathrm{~B}$
374
13
4

167

3.1

$\begin{array}{ll}158 . & 3.0 \\ .81 & .46\end{array}$

$\begin{array}{rr}.15 & .27 \\ 8 & .27\end{array}$

8.2

.78

$290 \quad 192$

$\begin{array}{rc}150 & 5.7 \\ 136 & 4.6 \\ 146 & 4.5 \\ 2.040 & 18 \\ 1,750 & 16 \\ 7,340 & 40 \\ 99 & 1.5 \\ 2.2 & 1.1 \\ 4.5 & .68 \\ .12 & .41\end{array}$

$194 \quad 21$

237

233

914
827

212

232
827

827
$7 \mathrm{~B} 4$

2,060

1,320

484

14
48
2

$.12 \quad .43$

2
15
5

5
10
5

.85

1.3

$.36 \quad .86$

$5.3 \quad 1.2$

$\begin{array}{cc}.39 & .23 \\ .95 & .31 \\ 6.8 & .49\end{array}$

JULY $25 \quad 0940$

\begin{tabular}{l|ll} 
JULY & 25 & 0940 \\
JULY & 29 & 1310
\end{tabular}

225

AUG $\begin{array}{rrrr}1 & 1125 & 225 \\ & 1 & 230\end{array}$

AUG $9 \quad 1140 \quad 260$

AUG $\quad 16 \quad 0830$

220

AUG $19 \quad 1340 \quad 215$

AUG $23 \quad 1145 \quad 210$

AUG 2B 0935

AUG $28 \quad 1000$

AUG , $30 \quad 1250$

160

SEPT $3 \quad 1300$

$1300-240$

SEPT $9 \quad 1420 \quad 220$

\begin{tabular}{l|lll} 
SEPT & 12 & 1830 & 150
\end{tabular}

SEPT $12 \quad 1835 \quad 150$

SEPT $12 \quad 1840$

SEPT $12 \quad 1850$

SEPT $12 \quad 1900$

SEPT $12 \quad 1910$

SEPI $12 \quad 1920$

\begin{tabular}{l|ll} 
SEPT & $12 \quad 1930$
\end{tabular}

\begin{tabular}{l|ll} 
SEPI & $12 \quad 1945$
\end{tabular}

SEPT $12 \quad 2000$

SEPT $12 \quad 2015$

SEPT $12 \quad 2030$

130

120

120

100
110

$.96 \quad .41$

$\begin{array}{ll}.62 & .41 \\ .76 & .49\end{array}$

$\begin{array}{ll}.76 & .39 \\ .93 & .45\end{array}$

$.47 \quad .31$

SEPT $12 \quad 2045$
$110 \quad 2,940$

$110 \quad 3,690$

$120 \cdot 4,150$

$\begin{array}{ll}110 & 3,910 \\ 110 & 3,560\end{array}$

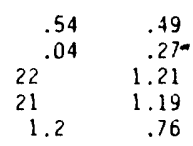

$.26 \quad .31$<smiles>C1CCCCC1</smiles>

1.5

2,240

2,420

$10^{.21}$

1,550

1,920

3,880

1,910

1,610

2,030

6,350

7,010

10,600

$1,620 \quad 12,900$

$1,320 \quad 13,200$

$1,180 \quad 13,200$

$\begin{array}{ll}1,060 & 11,200 \\ 1,060 & 10,200\end{array}$
11

16

24

38

44

57

72

81
76
69 


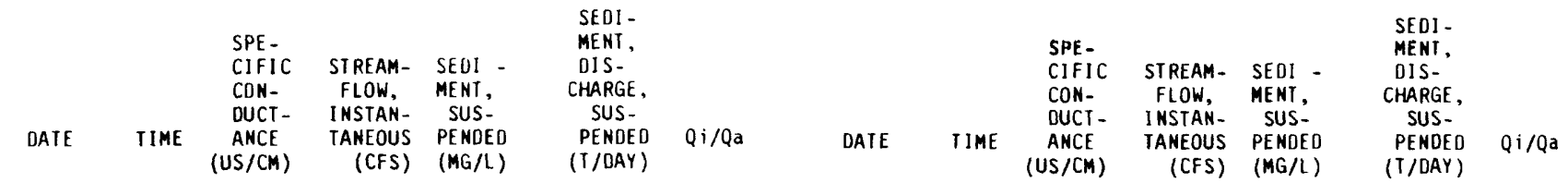

WATER YEAR DCTOBER 1984 TO SEPTEMBER 1985-Cont inued WATER YEAR OCTOBER 1985 TD SEPTEMBER 19B6

$\begin{array}{llllllll}\text { SEPI } & 12 & 2100 & 120 & 3,130 & 1,030 & B, 70 D & 61 \\ \text { SEPT } & 12 & 2115 & 120 & 2, B B 0 & 1,2 B 0 & 9,950 & 56 \\ \text { SEPT } & 12 & 2130 & 120 & 2,790 & 1,210 & 9,110 & 54 \\ \text { SEPT } & 12 & 2200 & 110 & 2, B 10 & 1,020 & 7,740 & 55 \\ \text { SEPT } & 12 & 2230 & 120 & 3,040 & 855 & 7,020 & 69 \\ & & & & & & & \end{array}$

61
56
54
55
69

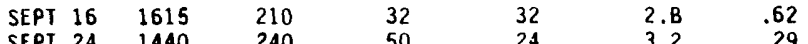

WATER YEAR OCTOBER 19B5 TO SEPTEMBER 1986

\begin{tabular}{|c|c|c|c|c|c|c|c|}
\hline $\begin{array}{l}\text { OCT } \\
\text { OCT } \\
\text { OCT } \\
\text { OCT } \\
\text { OCT }\end{array}$ & $\begin{array}{l}3 \\
6 \\
6 \\
6 \\
6\end{array}$ & $\begin{array}{l}1110 \\
0600 \\
0605 \\
0610 \\
0620\end{array}$ & $\begin{array}{r}180 \\
80 \\
B 0 \\
80 \\
80\end{array}$ & $\begin{array}{r}90 \\
484 \\
512 \\
540 \\
616\end{array}$ & $\begin{array}{r}178 \\
1,930 \\
1,870 \\
1,600 \\
2,880\end{array}$ & $\begin{array}{r}43 \\
2,520 \\
2,590 \\
2,340 \\
4,800\end{array}$ & $\begin{array}{r}1 . \\
9 . \\
10 \\
10 \\
12\end{array}$ \\
\hline $\begin{array}{l}O C T \\
O C T \\
O C T \\
O C T \\
0 C T\end{array}$ & $\begin{array}{l}6 \\
6 \\
6 \\
6 \\
6\end{array}$ & $\begin{array}{l}0630 \\
0640 \\
0650 \\
0700 \\
0715\end{array}$ & $\begin{array}{l}8 D \\
80 \\
80 \\
80 \\
B 0\end{array}$ & $\begin{array}{r}712 \\
842 \\
964 \\
1,080 \\
1,270\end{array}$ & $\begin{array}{l}1,930 \\
1,580 \\
1,460 \\
1,380 \\
1,200\end{array}$ & $\begin{array}{l}3,720 \\
3.600 \\
3.810 \\
4.020 \\
4.130\end{array}$ & $\begin{array}{l}14 \\
16 \\
19 \\
21 \\
25\end{array}$ \\
\hline $\begin{array}{l}O C T \\
O C T \\
O C T \\
O C T \\
O C T\end{array}$ & $\begin{array}{l}6 \\
6 \\
6 \\
6 \\
6\end{array}$ & $\begin{array}{l}0730 \\
0745 \\
0800 \\
0815 \\
0830\end{array}$ & $\begin{array}{l}80 \\
80 \\
80 \\
70 \\
70\end{array}$ & $\begin{array}{l}1,430 \\
1,600 \\
1,670 \\
2,100 \\
2,650\end{array}$ & $\begin{array}{l}1,000 \\
1,270 \\
2,320 \\
2,480 \\
2,380\end{array}$ & $\begin{array}{r}3,860 \\
5,470 \\
10,500 \\
14,100 \\
17,000\end{array}$ & $\begin{array}{l}28 \\
31 \\
32 \\
41 \\
52\end{array}$ \\
\hline $\begin{array}{l}O C T \\
O C T \\
O C T \\
O C T \\
O C T\end{array}$ & $\begin{array}{l}6 \\
6 \\
6 \\
6 \\
6\end{array}$ & $\begin{array}{l}0845 \\
0900 \\
0930 \\
1000 \\
1030\end{array}$ & $\begin{array}{l}70 \\
70 \\
70 \\
70 \\
70\end{array}$ & $\begin{array}{l}3,220 \\
3,690 \\
3,800 \\
3,250 \\
2,890\end{array}$ & $\begin{array}{l}2,480 \\
2,500 \\
1,760 \\
1,350 \\
1,980\end{array}$ & $\begin{array}{l}21,600 \\
24,900 \\
18,100 \\
11,800 \\
15,400\end{array}$ & $\begin{array}{l}63 \\
72 \\
74 \\
63 \\
56\end{array}$ \\
\hline $\begin{array}{l}O C T \\
O C I \\
O C T \\
O C T \\
O C T\end{array}$ & $\begin{array}{l}6 \\
6 \\
6 \\
6 \\
6\end{array}$ & $\begin{array}{l}1100 \\
1130 \\
1200 \\
1230 \\
1300\end{array}$ & $\begin{array}{l}70 \\
70 \\
70 \\
70 \\
70\end{array}$ & $\begin{array}{l}5,410 \\
7,190 \\
5,770 \\
4.350 \\
3,970\end{array}$ & $\begin{array}{l}1,720 \\
2,000 \\
1,590 \\
1,150 \\
1,140\end{array}$ & $\begin{array}{l}25,100 \\
3 B, 800 \\
24,800 \\
13,500 \\
12,200\end{array}$ & $\begin{array}{r}105 \\
140 \\
112 \\
85 \\
77\end{array}$ \\
\hline $\begin{array}{l}O C T \\
O C T \\
O C T \\
O C T \\
O C T\end{array}$ & $\begin{array}{l}6 \\
6 \\
6 \\
6 \\
6\end{array}$ & $\begin{array}{l}1320 \\
1325 \\
1330 \\
1340 \\
1350\end{array}$ & $\begin{array}{l}140 \\
140 \\
140 \\
140 \\
130\end{array}$ & $\begin{array}{l}4,14 D \\
4,220 \\
4,290 \\
4,350 \\
4,400\end{array}$ & $\begin{array}{l}380 \\
404 \\
419 \\
463 \\
514\end{array}$ & $\begin{array}{l}4,250 \\
4,600 \\
4,850 \\
5,440 \\
6,110\end{array}$ & $\begin{array}{l}81 \\
82 \\
84 \\
85 \\
86\end{array}$ \\
\hline $\begin{array}{l}0 C T \\
O C T \\
0 C T \\
0 C T \\
O C T\end{array}$ & $\begin{array}{l}6 \\
6 \\
6 \\
6 \\
6\end{array}$ & $\begin{array}{l}1400 \\
1410 \\
1420 \\
1435 \\
1450\end{array}$ & $\begin{array}{l}130 \\
130 \\
130 \\
130 \\
130\end{array}$ & $\begin{array}{l}4,440 \\
4,420 \\
4,320 \\
4,100 \\
4,090\end{array}$ & $\begin{array}{l}637 \\
662 \\
659 \\
809 \\
868\end{array}$ & $\begin{array}{l}7,640 \\
7,900 \\
7,690 \\
8,960 \\
9,590\end{array}$ & $\begin{array}{l}86 \\
86 \\
84 \\
80 \\
80\end{array}$ \\
\hline $\begin{array}{l}O C T \\
O C T \\
O C T \\
O C T \\
O C I\end{array}$ & $\begin{array}{l}6 \\
6 \\
6 \\
6 \\
6\end{array}$ & $\begin{array}{l}1505 \\
1520 \\
1535 \\
1550 \\
1605\end{array}$ & $\begin{array}{r}130 \\
130 \\
130 \\
130 \\
90\end{array}$ & $\begin{array}{l}4,500 \\
5,260 \\
5,910 \\
6,190 \\
6,040\end{array}$ & $\begin{array}{r}942 \\
898 \\
1,160 \\
2,240 \\
3,080\end{array}$ & $\begin{array}{l}11,400 \\
12,800 \\
18,500 \\
37,400 \\
50,200\end{array}$ & $\begin{array}{r}88 \\
102 \\
115 \\
120 \\
118\end{array}$ \\
\hline $\begin{array}{l}O C T \\
O C T \\
O C T \\
O C T \\
O C T\end{array}$ & $\begin{array}{l}6 \\
6 \\
6 \\
6 \\
6\end{array}$ & $\begin{array}{l}1620 \\
1650 \\
1720 \\
1750 \\
1820\end{array}$ & $\begin{array}{l}90 \\
90 \\
90 \\
90 \\
.90\end{array}$ & $\begin{array}{l}5,600 \\
4,680 \\
4,070 \\
4,750 \\
5,220\end{array}$ & $\begin{array}{l}4,200 \\
2,480 \\
1,570 \\
1,240 \\
2,920\end{array}$ & $\begin{array}{l}63,500 \\
31.300 \\
17,300 \\
15,900 \\
41,100\end{array}$ & $\begin{array}{r}109 \\
91 \\
79 \\
92 \\
102\end{array}$ \\
\hline $\begin{array}{l}O C T \\
O C T \\
O C T \\
O C T \\
O C T\end{array}$ & $\begin{array}{l}6 \\
6 \\
6 \\
6 \\
7\end{array}$ & $\begin{array}{l}1850 \\
1920 \\
1950 \\
2020 \\
1200\end{array}$ & $\begin{array}{l}65 \\
60 \\
80 \\
70 \\
95\end{array}$ & $\begin{array}{r}4,900 \\
4,000 \\
3,200 \\
3,010 \\
725\end{array}$ & $\begin{array}{l}5,740 \\
3,950 \\
3,460 \\
2,140 \\
1,320\end{array}$ & $\begin{array}{r}76,000 \\
42,700 \\
29,900 \\
17,400 \\
9,980\end{array}$ & $\begin{array}{l}96 \\
78 \\
62 \\
59 \\
14\end{array}$ \\
\hline
\end{tabular}

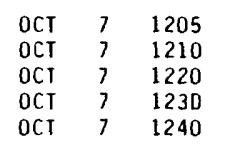

OCT 71250

$\begin{array}{lll}\text { OCI } 7 & 1300 \\ \text { OCT } & 7 & 1315\end{array}$

OCT 71315

$\begin{array}{lll}\text { OCT } 7 & 1330 \\ \text { OCI } & 7 & 1345\end{array}$

OCT $7 \quad 1400$

$\begin{array}{lll}\text { OCT } & 7 & 1415 \\ \text { DCT } & 7 & 1430\end{array}$

$\begin{array}{lll}\text { DCT } & 7 & 1430 \\ \text { OCT } & 8 & 1235\end{array}$

OCT 81331

OCI $15 \quad 1155$

$\begin{array}{lll}\text { OCT } & 23 & 1315 \\ \text { OCT } & 23 & 1320\end{array}$

$\begin{array}{lll}\text { OCT } & 23 & 1320 \\ \text { OCT } & 23 & 1325\end{array}$

OCT $23 \quad 1335$

OCI $23 \quad 1345$

OCT $23 \quad 1355$

OCI $23 \quad 1405$

$\begin{array}{lll}\text { OCI } & 23 & 1415 \\ \text { OCT } & 23 & 1430\end{array}$

OCT $23 \quad 1445$

$\begin{array}{lll}\text { OCT } & 23 & 1500 \\ \text { OCT } & 23 & 1515\end{array}$

$\begin{array}{lll}\text { OCT } & 23 & 1515 \\ \text { OCT } & 23 & 1530\end{array}$

OCT $23 \quad 1545$

OCT $23 \quad 1600$

OCT $24 \quad 1255$

OCT $24 \quad 1300$

OCT $24 \quad 1305$

OCT $24 \quad 1315$

OCI $24 \quad 1325$

OCT. $24 \quad 1335$

OCT $24 \quad 1510$

OCT $24 \quad 1525$

OCT $24 \quad 1540$

OCI $24 \quad 1555$

$\begin{array}{lll}O C T & 24 & 1625\end{array}$

OCT $24 \quad 1655$

OCT $24 \quad 1725$

OCI $24 \quad 1755$

$\begin{array}{lll}\text { OCT } & 24 & 1825 \\ \text { OCT } & 24 & 1855\end{array}$

OCT $24 \quad 1925$

OCT $24 \quad 1955$

NOV $15 \quad 0900$

NOV $15 \quad 0905$

NOV $15 \quad 0910$

NOV $15 \quad 0920$

NOV 150930

NOV $15 \quad 1015$

NOV $15 \quad 1030$

NOV $15 \quad 1045$

NOV $15 \quad 1100$

NOV $15 \quad 1115$

MOV $15 \quad 1130$

$\begin{array}{ll}95 & 7 \\ 95 & 7 \\ 95 & 7 \\ 95 & 6 \\ 95 & 604\end{array}$

725
725
711
682
604

$95 \quad 554$

$\begin{array}{ll}95 & 534 \\ 95 & 505\end{array}$

100

100

100

105

105
135

135

210

150
155

175

160

155
150
140

130
130

130

$\begin{array}{ll}120 & 2,120 \\ 115 & 1,890 \\ 120 & 1,740 \\ 110 & 1,650 \\ 110 & 1,580\end{array}$

$110 \quad 1,490$

$\begin{array}{ll}100 & 540 \\ 100 & 530\end{array}$

$100 \quad 506$

$100 \quad 456$

$100 \quad 434$

$100 \quad 409$

100

110
110

463
536

714

1101,090

$100 \quad 1,810$

$90 \quad 1,790$

753,860

$60 \quad 5,660$

$\begin{array}{ll}50 & 3,760 \\ 50 & 2,910 \\ 50 & 1,970 \\ 60 & 1,950\end{array}$

$100 \quad 494$

$\begin{array}{rr}80 & 483 \\ 80 & 472 \\ 90 & 450 \\ 100 & 430 \\ 110 & 644\end{array}$

483

472
450

430

$\begin{array}{lr}110 & 921 \\ 110 & 1,220\end{array}$

$100 \quad 1,520$

$\begin{array}{rr}100 & 1,720 \\ 90 & 1,920\end{array}$

$\begin{array}{rrr}78 & 153 & 14 \\ 24 & 47 & 14 \\ 32 & 61 & 14 \\ 26 & 47 & 13 \\ 47 & 76 & 12\end{array}$

$\begin{array}{rrr}42 & 63 & 11 \\ 1,170 & 1,680 & 10 \\ 59 & 81 & 10 \\ 83 & 107 & 9\end{array}$

$\begin{array}{lll}85 & 110 & 9.4\end{array}$

$\begin{array}{lll}52 & 62 & 8.5 \\ 36 & 41 & 8.3 \\ 26 & 28 & 8.0 \\ 88 & 47 & 3.8 \\ 95 & 48 & 3.7\end{array}$

$\begin{array}{rrr}10 & 1.6 & 1.2 \\ 1,350 & 1,820 & 9.8 \\ 1,390 & 2,370 & 12 \\ 1,270 & 2,620 & 15 \\ 1,270 & 3,750 & 21\end{array}$

$\begin{array}{lll}1,270 & 3,750 & 21\end{array}$

$\begin{array}{rrr}570 & 2,350 & 30 \\ 1,260 & 7,100 & 40 \\ 1,290 & 8,210 & 46 \\ 1,160 & 7,440 & 46 \\ 1,000 & 6,400 & 46\end{array}$

7354,210

$\begin{array}{lll}67 \mathrm{~B} & 3,460 & 37 \\ 817 & 3, \mathrm{~B} 40 & 34\end{array}$

$\begin{array}{lll}815 & 3,630 & 32 \\ 749 & 3,200\end{array}$

$878 \quad 3,530 \quad 29$

$\begin{array}{lll}795 & 1,160 & 29 \\ 454 & 649 & 10\end{array}$

$\begin{array}{lll}454 & 549 & 10\end{array}$

$\begin{array}{lll}382 & 470 & 8.9\end{array}$

$\begin{array}{lll}337 & 396 & 8.4\end{array}$

$\begin{array}{lll}303 & 334 & 8.0 \\ 202 & 252 & 9.0\end{array}$

$\begin{array}{lll}270 & 391 & 10 \\ 287 & 553 & 14\end{array}$

$435 \quad 1,290 \quad 21$

$\begin{array}{lll}1,110 & 5,440 & 35 \\ 1,130 & 5,450 & 35\end{array}$

$1,790 \quad 18,600 \quad 75$

$5,190 \quad 79,300 \quad 110$

$\begin{array}{lll}5,020 & 51.000 & 73\end{array}$

$\begin{array}{rrr}2,640 & 20,700 & 57 \\ 1,280 & 6,830 & 38\end{array}$

$\begin{array}{rrr}1,280 & 6,830 & 38 \\ 987 & 5,200 & 3 \mathrm{~B} \\ 2,090 & 2,780 & 9 .\end{array}$

$2.520 \quad 3,290 \quad 9.4$

$\begin{array}{lll}2,520 & 3,290 & 9.4 \\ 2,120 & 2,700 & 9.2\end{array}$

$\begin{array}{lll}1,690 & 2,060 & 8.8 \\ 1,520 & 1,760 & 8.4\end{array}$

$1,320 \quad 2,300 \quad 12$

$2,080 \quad 5,180 \quad 18$

$2,140 \quad 7,070 \quad 24$

$2,150 \quad 8,830 \quad 30$

$\begin{array}{lll}1,850 & 8,600 & 33 \\ 1,770 & 9,200 & 37\end{array}$ 


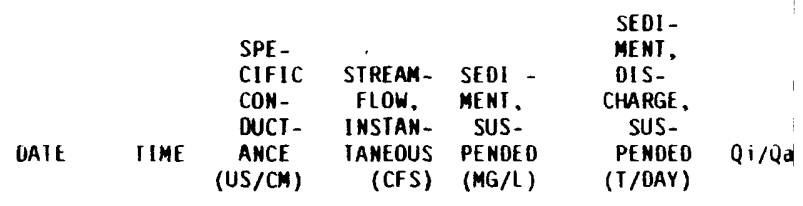

WATER YEAR OCTOBER 1985 TO SEPIEMBER 1986 -Cont InUed

\begin{tabular}{|c|c|c|c|c|c|c|c|}
\hline $\begin{array}{l}\text { Nov } \\
\text { nOv } \\
\text { NOV } \\
\text { NOV } \\
\text { NOV }\end{array}$ & $\begin{array}{l}15 \\
15 \\
15 \\
15 \\
15\end{array}$ & $\begin{array}{l}1145 \\
1200 \\
1230 \\
1300 \\
1330\end{array}$ & $\begin{array}{l}90 \\
90 \\
90 \\
90 \\
90\end{array}$ & $\begin{array}{r}1,740 \\
1.560 \\
1,260 \\
973 \\
780\end{array}$ & $\begin{array}{r}1.570 \\
1,480 \\
927 \\
842 \\
130\end{array}$ & $\begin{array}{l}7,360 \\
6,250 \\
3,150 \\
2,210 \\
1,540\end{array}$ & $\begin{array}{l}34 \\
30 \\
24 \\
19 \\
15\end{array}$ \\
\hline $\begin{array}{l}\text { NOV } \\
\text { NOV } \\
\text { NOV } \\
\text { NOV } \\
\text { NOV }\end{array}$ & $\begin{array}{l}15 \\
15 \\
15 \\
15 \\
12\end{array}$ & $\begin{array}{l}1400 \\
1430 \\
1500 \\
1530 \\
1225\end{array}$ & $\begin{array}{l}100 \\
100 \\
100 \\
110 \\
220\end{array}$ & $\begin{array}{r}686 \\
610 \\
534 \\
480 \\
52\end{array}$ & $\begin{array}{r}650 \\
464 \\
397 \\
354 \\
27\end{array}$ & $\begin{array}{r}1,210 \\
764 \\
572 \\
459 \\
3.8\end{array}$ & $\begin{array}{c}13 \\
12 \\
10 \\
9.3 \\
1.0\end{array}$ \\
\hline $\begin{array}{l}\text { NOV } \\
\text { NOV } \\
\text { NOV } \\
\text { DEC } \\
\text { OEC }\end{array}$ & $\begin{array}{r}14 \\
22 \\
27 \\
2 \\
6\end{array}$ & $\begin{array}{l}1140 \\
1125 \\
1045 \\
1045 \\
1215\end{array}$ & $\begin{array}{l}270 \\
230 \\
220 \\
250 \\
240\end{array}$ & $\begin{array}{l}43 \\
41 \\
31 \\
31 \\
31\end{array}$ & $\begin{array}{r}24 \\
11 \\
7 \\
6 \\
13\end{array}$ & $\begin{array}{r}2.8 \\
1.2 \\
.6 \\
.6 \\
1.1\end{array}$ & $\begin{array}{l}.84 \\
.80 \\
.60 \\
.60 \\
.60\end{array}$ \\
\hline $\begin{array}{l}\text { DEC } \\
\text { DEC } \\
\text { OEC } \\
\text { OEC } \\
\text { JAN }\end{array}$ & $\begin{array}{r}9 \\
12 \\
16 \\
23 \\
8\end{array}$ & $\begin{array}{l}1200 \\
0955 \\
1325 \\
1120 \\
1150\end{array}$ & $\begin{array}{l}250 \\
250 \\
250 \\
250 \\
250\end{array}$ & $\begin{array}{l}26 \\
41 \\
22 \\
22 \\
17\end{array}$ & $\begin{array}{r}13 \\
26 \\
10 \\
7 \\
6\end{array}$ & $\begin{array}{l}.91 \\
2.9 \\
.59 \\
.41 \\
.28\end{array}$ & $\begin{array}{l}.51 \\
.80 \\
.43 \\
.43 \\
.33\end{array}$ \\
\hline $\begin{array}{l}\text { JAN } \\
\text { JAN } \\
\text { JAN } \\
\text { JAN } \\
\text { JAN }\end{array}$ & $\begin{array}{l}13 \\
16 \\
21 \\
23 \\
27\end{array}$ & $\begin{array}{l}1345 \\
0940 \\
1225 \\
1045 \\
0935\end{array}$ & $\begin{array}{l}250 \\
260 \\
255 \\
260 \\
270\end{array}$ & $\begin{array}{l}18 \\
15 \\
21 \\
16 \\
14\end{array}$ & $\begin{array}{r}4 \\
5 \\
6 \\
5 \\
14\end{array}$ & $\begin{array}{l}.19 \\
.20 \\
.34 \\
.22 \\
.53\end{array}$ & $\begin{array}{l}.35 \\
.29 \\
.41 \\
.31 \\
.21\end{array}$ \\
\hline $\begin{array}{l}\text { FEB } \\
\text { FEB } \\
\text { FEB } \\
\text { FEB } \\
\text { FEB }\end{array}$ & $\begin{array}{r}3 \\
10 \\
13 \\
18 \\
27\end{array}$ & $\begin{array}{l}1155 \\
1210 \\
1055 \\
1045 \\
0810\end{array}$ & $\begin{array}{l}270 \\
270 \\
270 \\
260 \\
270\end{array}$ & $\begin{array}{l}12 \\
12 \\
11 \\
12 \\
10\end{array}$ & $\begin{array}{l}2 \\
2 \\
2 \\
3 \\
1\end{array}$ & $\begin{array}{l}.06 \\
.06 \\
.06 \\
.10 \\
.03\end{array}$ & $\begin{array}{l}.23 \\
.23 \\
.21 \\
.23 \\
.19\end{array}$ \\
\hline $\begin{array}{l}\text { MAR } \\
\text { MAR } \\
\text { MAR } \\
\text { MAR } \\
\text { MAR }\end{array}$ & $\begin{array}{r}3 \\
17 \\
20 \\
24 \\
31\end{array}$ & $\begin{array}{l}1245 \\
1225 \\
0945 \\
1115 \\
1210\end{array}$ & $\begin{array}{l}280 \\
310 \\
290 \\
280 \\
260\end{array}$ & $\begin{array}{l}9.4 \\
9.7 \\
9.1 \\
7.7 \\
33\end{array}$ & $\begin{array}{r}2 \\
8 \\
2 \\
6 \\
91\end{array}$ & $\begin{array}{c}.05 \\
.20 \\
.05 \\
.12 \\
8.1\end{array}$ & $\begin{array}{l}.18 \\
.19 \\
.18 \\
.15 \\
.64\end{array}$ \\
\hline $\begin{array}{l}\text { APR } \\
\text { APR } \\
\text { APR } \\
\text { APR } \\
\text { APR }\end{array}$ & $\begin{array}{r}3 \\
10 \\
14 \\
17 \\
21\end{array}$ & $\begin{array}{l}1105 \\
1115 \\
1210 \\
0900 \\
1150\end{array}$ & $\begin{array}{l}280 \\
260 \\
290 \\
290 \\
290\end{array}$ & $\begin{array}{c}13 \\
14 \\
10 \\
9.1 \\
8.5\end{array}$ & $\begin{array}{r}8 \\
29 \\
4 \\
6 \\
4\end{array}$ & $\begin{array}{c}.28 \\
1.1 \\
.11 \\
.15 \\
.09\end{array}$ & $\begin{array}{l}.2 \\
.2\end{array}$ \\
\hline $\begin{array}{l}\text { APR } \\
\text { APR } \\
\text { APR } \\
\text { APR } \\
\text { APR }\end{array}$ & $\begin{array}{l}29 \\
29 \\
29 \\
29 \\
29\end{array}$ & $\begin{array}{l}1535 \\
1540 \\
1545 \\
1605 \\
1615\end{array}$ & $\begin{array}{l}230 \\
220 \\
210 \\
130 \\
130\end{array}$ & $\begin{array}{r}744 \\
1,410 \\
2,070 \\
2,580 \\
2,700\end{array}$ & $\begin{array}{l}1,050 \\
3,250 \\
3,020 \\
2,830 \\
3,040\end{array}$ & $\begin{array}{r}2,110 \\
12,400 \\
16,900 \\
19,700 \\
22,200\end{array}$ & $\begin{array}{l}14 \\
27 \\
40 \\
50 \\
53\end{array}$ \\
\hline $\begin{array}{l}\text { APR } \\
\text { APR } \\
\text { APR } \\
\text { APR } \\
\text { APR }\end{array}$ & $\begin{array}{l}29 \\
29 \\
29 \\
29 \\
29\end{array}$ & $\begin{array}{l}1625 \\
1635 \\
1650 \\
1705 \\
1720\end{array}$ & $\begin{array}{l}130 \\
110 \\
110 \\
130 \\
110\end{array}$ & $\begin{array}{l}2,740 \\
2,680 \\
2,420 \\
2,090 \\
1,780\end{array}$ & $\begin{array}{l}2.750 \\
3,250 \\
2.250 \\
1.840 \\
1,470\end{array}$ & $\begin{array}{r}20,300 \\
23,500 \\
14,700 \\
10,400 \\
7,060\end{array}$ & $\begin{array}{l}53 \\
52 \\
47 \\
41 \\
35\end{array}$ \\
\hline $\begin{array}{l}A P R \\
A P R \\
A P R \\
A P R \\
A P R \\
A P R\end{array}$ & $\begin{array}{l}29 \\
29 \\
29 \\
29 \\
29\end{array}$ & $\begin{array}{l}1735 \\
1750 \\
1805 \\
1820 \\
1835\end{array}$ & $\begin{array}{l}120 \\
130 \\
120 \\
130 \\
140\end{array}$ & $\begin{array}{r}1,580 \\
1,390 \\
1,230 \\
1,077 \\
991\end{array}$ & $\begin{array}{r}1,490 \\
1,320 \\
1,190 \\
1,110 \\
968\end{array}$ & $\begin{array}{l}6,350 \\
4,950 \\
3,950 \\
3,220 \\
2,590\end{array}$ & $\begin{array}{l}31 \\
27 \\
24 \\
21 \\
19\end{array}$ \\
\hline $\begin{array}{l}A P R \\
A P R \\
A P R \\
A P R \\
\text { MAY }\end{array}$ & $\begin{array}{l}29 \\
29 \\
29 \\
29 \\
5\end{array}$ & $\begin{array}{l}1850 \\
1920 \\
1950 \\
2020 \\
1155\end{array}$ & $\begin{array}{l}130 \\
130 \\
140 \\
150 \\
270\end{array}$ & $\begin{array}{r}888 \\
726 \\
596 \\
496 \\
13\end{array}$ & $\begin{array}{r}863 \\
754 \\
639 \\
494 \\
?\end{array}$ & $\begin{array}{r}2,070 \\
1,480 \\
1,030 \\
661\end{array}$ & $\begin{array}{c}17 \\
14 \\
12 \\
9.7\end{array}$ \\
\hline
\end{tabular}

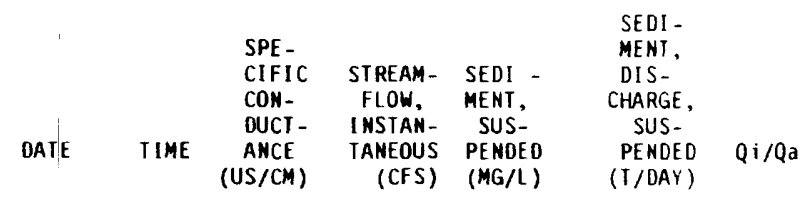

WAIER YEAR OCTOBER 1985 TO SEPTEMBER 1986

\begin{tabular}{|c|c|c|c|c|c|c|c|}
\hline MAY & 8 & 1215 & 270 & 21 & 104 & 5.9 & 0.41 \\
\hline MAY & 10 & 1745 & 170 & 665 & 491 & 881 & 13 \\
\hline MAY & 10 & 1750 & 180 & 719 & 603 & 1.170 & 14 \\
\hline MAY & 10 & 1755 & 180 & 773 & 902 & 1.880 & 15 \\
\hline MAY & 10 & 1805 & 175 & 848 & 873 & 2,000 & 16 \\
\hline MAY & 10 & 1815 & 175 & 891 & 832 & 2,000 & 17 \\
\hline MAY & 10 & 1825 & 155 & 901 & 710 & 1,730 & 18 \\
\hline MAY & 10 & 1835 & 150 & 901 & 670 & 1,630 & 18 \\
\hline MAY & 10 & 1845 & 140 & 891 & 712 & 1,710 & 17 \\
\hline MAY & 10 & 1900 & 140 & 931 & 742 & 1,870 & 18 \\
\hline MAY & 10 & 1915 & 1.0 & 978 & 551 & 1,450 & 19 \\
\hline MAY & 10 & 1930 & 135 & 994 & 450 & 1,210 & 19 \\
\hline MAY & 10 & 1945 & 140 & 1,100 & 390 & 1,160 & 21 \\
\hline MAY & 10 & 2000 & 135 & 1,050 & 394 & 1,120 & 20 \\
\hline MAY & 10 & 2015 & 140 & 1,060 & 379 & 1,080 & 21 \\
\hline MAY & 10 & 2030 & 140 & 1,050 & 373 & 1,060 & 20 \\
\hline MAY & 10 & 2045 & 140 & 1,030 & 371 & 1,030 & 20 \\
\hline MAY & 10 & 2115 & 140 & 916 & 305 & 754 & 18 \\
\hline MAY & 10 & 2145 & 130 & 886 & $28 \mathrm{~B}$ & 690 & 17 \\
\hline MAY & 10 & 2215 & 130 & 822 & 246 & 546 & 16 \\
\hline MAY & 10 & 2245 & 150 & 789 & 232 & 490 & 15 \\
\hline MAY & 10 & 2315 & 150 & 761 & 167 & 342 & 15 \\
\hline MAY & 10 & 2345 & 150 & 734 & 147 & 291 & 14 \\
\hline MAY & 11 & 0015 & 150 & 708 & 432 & 826 & 14 \\
\hline MAY & 11 & 0045 & 145 & 712 & 435 & 836 & 14 \\
\hline MAY & 13 & 0300 & 120 & 587 & 1,810 & 2,870 & 11 \\
\hline MAY & 13 & 0305 & 120 & 841 & 1,520 & 3,460 & 16 \\
\hline MAY & 13 & 0310 & 120 & 1,100 & 1,480 & 4,400 & 21 \\
\hline MAY & 13 & 0320 & 160 & 1,410 & 1,950 & 7,440 & 27 \\
\hline MAY & 13 & 0330 & 160 & 1,540 & 1,630 & 6,770 & 30 \\
\hline MAY & 13 & 0340 & 160 & 1,420 & 1,330 & 5,110 & 28 \\
\hline MAY & 13 & 0350 & 145 & 1,390 & 1,050 & 3,940 & 27 \\
\hline MAY & 13 & 0400 & 130 & 1,270 & 825 & 2,830 & 25 \\
\hline MAY & 13 & 0415 & 130 & 1,130 & 706 & 2,150 & 22 \\
\hline MAY & 13 & 0430 & 130 & 994 & 611 & 1.640 & 19 \\
\hline MAY & 13 & 0445 & 130 & 871 & 558 & 1,310 & 17 \\
\hline MAY & 13 & 0500 & 130 & 808 & 505 & 1,100 & 16 \\
\hline MAY & 13 & 0515 & 130 & 708 & 559 & 1,070 & 14 \\
\hline MAY & 13 & 0530 & 130 & 635 & 554 & 950 & 12 \\
\hline MAY & 13 & 0545 & 140 & 512 & 525 & 726 & 10 \\
\hline MAY & 13 & 0600 & 140 & 505 & 549 & 749 & 9.8 \\
\hline MAY & 13 & 0630 & 140 & 449 & 482 & 584 & 8.8 \\
\hline MAY & 13 & 0730 & 140 & 798 & 529 & 1,140 & 16 \\
\hline MAY & 13 & 0800 & 140 & 1,610 & 723 & 3,140 & 31 \\
\hline MAY & 13 & 0830 & 110 & 4,100 & 8.970 & 99,300 & 80 \\
\hline MAY & 13 & 0900 & 110 & 3,790 & 4,400 & 45,000 & 74 \\
\hline MAY & 13 & 0930 & 110 & 3,590 & 3,640 & 35,300 & 70 \\
\hline MAY & 13 & 1000 & 110 & 2,740 & 2,360 & 17,500 & 53 \\
\hline MAY & 22 & 1115 & 270 & 113 & 174 & 53 & 2.2 \\
\hline JUNE & 2 & 1045 & 270 & 32 & 25 & 2.2 & .62 \\
\hline JUNE & 18 & 1100 & 250 & 53 & 31 & 4.4 & 1.0 \\
\hline JUNE & 23 & 1215 & 260 & 25 & 14 & .94 & .49 \\
\hline JULY & 1 & 1220 & 260 & 26 & 14 & .98 & .51 \\
\hline JULY & 7 & 1020 & 250 & 35 & 63 & 6.0 & .68 \\
\hline JULY & 14 & 1115 & 260 & 16 & 2 & .09 & .31 \\
\hline JULY & 29 & 1145 & 270 & 14 & 14 & .53 & .27 \\
\hline AUG & 4 & 1240 & 280 & 14 & 14 & .53 & .27 \\
\hline AUG & 12 & 1035 & 270 & 19 & 58 & 3.0 & .37 \\
\hline AUG & 19 & 1130 & 270 & 14 & 9 & .34 & .27 \\
\hline AUG & 26 & 1205 & 260 & 10 & 2 & .05 & .19 \\
\hline SEPI & 2 & 1240 & 270 & 17 & 38 & 1.7 & .33 \\
\hline SEPT & 8 & 1215 & 250 & 13 & 8 & .28 & .25 \\
\hline SEPT & 15 & 1045 & 250 & 12 & 1 & .03 & .23 \\
\hline SEPI & 22 & 1145 & 265 & 11 & 14 & .42 & .21 \\
\hline SEPT & 29 & 1225 & 280 & 24 & 6 & .39 & .47 \\
\hline
\end{tabular}




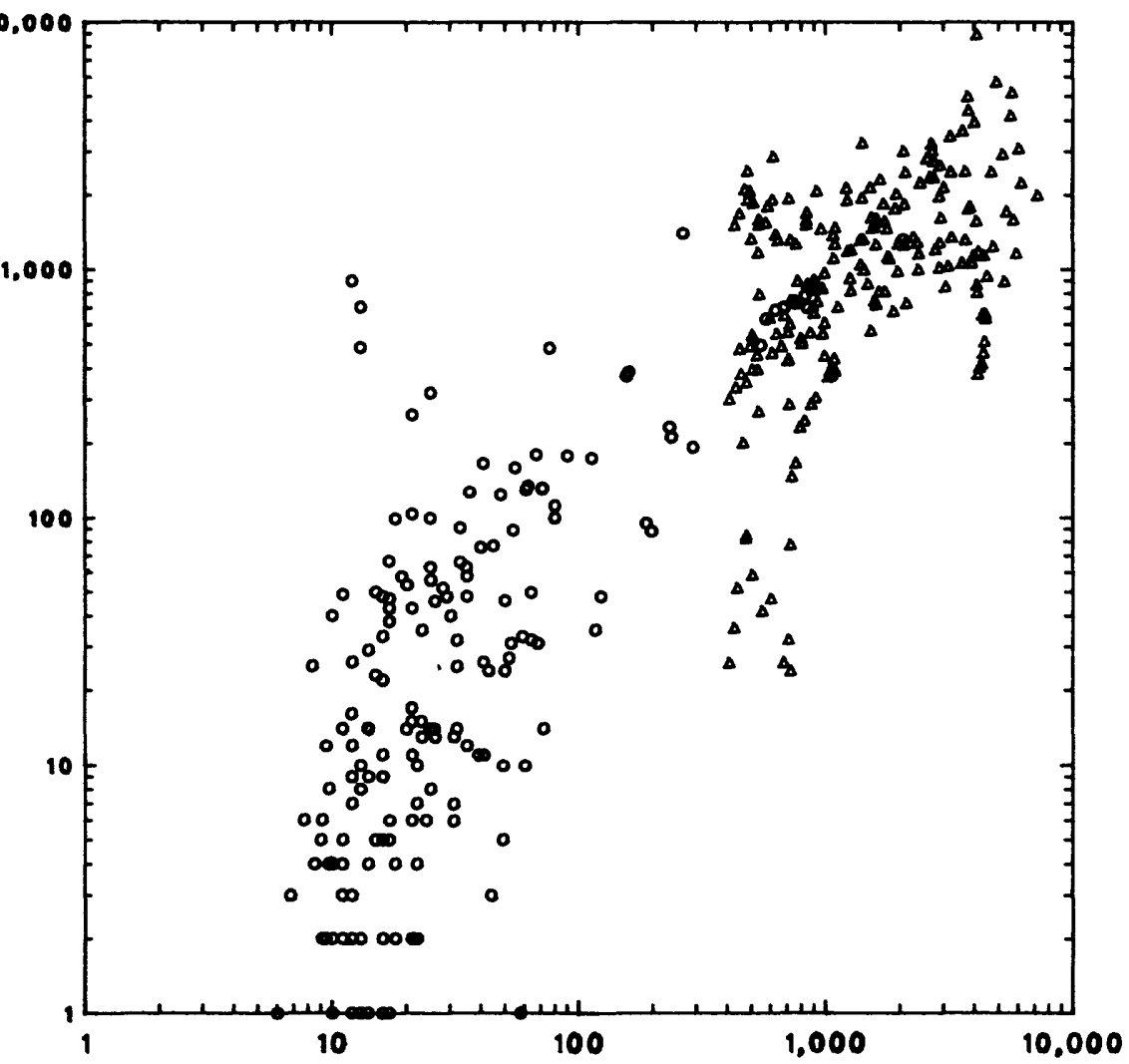

\section{EXPLANATION}

- FIELD SAMPLE

- ISCO SAMPLE

INSTANTANEOUS WATER DISCHARGE, IN CUBIC FEET PER SECOND

Figure 11.--Relation of suspended-sediment concentration to stream discharge for station Río Valenciano near Juncos. 
Iable 12. Suspended-sediment data for samples collected at station Quebrada Mamey near Gurabo

[US/CM, microsiemens per centimeter: CFS, Cubic foot per second; MG/L, milligrams per liter; I/DAY, tons per day; Qi/Qa, ratio of instantaneous water discharge to average water discharge; -.., missing data]

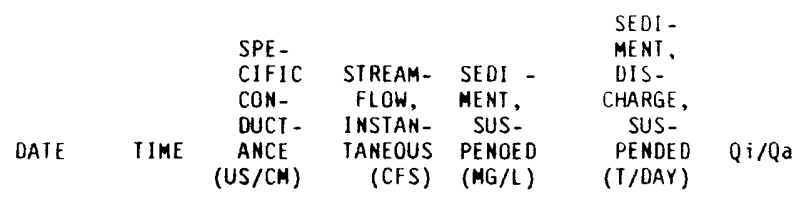
WATER YEAR OCTOBER 1983 TO SEPTEMBER 1984

\begin{tabular}{|c|c|c|c|c|c|c|c|}
\hline $\begin{array}{l}\text { FEB } \\
\text { FEB } \\
\text { FEB } \\
\text { FEB } \\
\text { FEB }\end{array}$ & $\begin{array}{l}9 \\
9 \\
9 \\
9 \\
9\end{array}$ & $\begin{array}{l}1345 \\
1410 \\
1415 \\
1430 \\
1440\end{array}$ & $\begin{array}{l}\cdots \\
\cdots \\
\cdots \\
\cdots \\
-. \\
30\end{array}$ & $\begin{array}{l}17 \\
18 \\
19 \\
19 \\
22\end{array}$ & $\begin{array}{l}344 \\
382 \\
442 \\
531 \\
692\end{array}$ & $\begin{array}{l}16 \\
19 \\
23 \\
27 \\
41\end{array}$ & $\begin{array}{l}3.9 \\
4.1 \\
4.3 \\
4.3 \\
5.0\end{array}$ \\
\hline $\begin{array}{l}\text { FEB } \\
\text { FEB } \\
\text { FEB } \\
\text { MAR } \\
\text { MAR }\end{array}$ & $\begin{array}{r}9 \\
9 \\
9 \\
8 \\
26\end{array}$ & $\begin{array}{l}1515 \\
1530 \\
1545 \\
1430 \\
1100\end{array}$ & $\begin{array}{l}303 \\
320 \\
337 \\
735 \\
690\end{array}$ & $\begin{array}{rrr}27 & \\
2 B & \\
25 & \\
1 & \\
& .51\end{array}$ & $\begin{array}{r}706 \\
422 \\
395 \\
0 \\
4\end{array}$ & $\begin{array}{r}51 \\
32 \\
27 \\
0 \\
\quad .01\end{array}$ & $\begin{array}{l}6.1 \\
6.4 \\
5.7 \\
.23 \\
.12\end{array}$ \\
\hline $\begin{array}{l}\text { APR } \\
\text { APR } \\
\text { MAY } \\
\text { MAY } \\
\text { MAY }\end{array}$ & $\begin{array}{l}10 \\
25 \\
21 \\
30 \\
30\end{array}$ & $\begin{array}{l}1115 \\
1230 \\
1430 \\
1440 \\
1445\end{array}$ & $\begin{array}{l}698 \\
760 \\
705 \\
442 \\
442\end{array}$ & $\begin{array}{l}.66 \\
.45 \\
.54 \\
5\end{array}$ & $\begin{array}{r}3 \\
0 \\
8 \\
81 \\
90\end{array}$ & $\begin{array}{c}.01 \\
.01 \\
1.1 \\
.97\end{array}$ & $\begin{array}{r}.15 \\
.10 \\
.12 \\
1.1 \\
.91\end{array}$ \\
\hline $\begin{array}{l}\text { JULY } \\
\text { AUG } \\
\text { AUG } \\
\text { SEPT } \\
\text { SEPI } \\
\text { SEPT }\end{array}$ & $\begin{array}{r}5 \\
28 \\
30 \\
14 \\
14 \\
19\end{array}$ & $\begin{array}{l}1430 \\
1420 \\
0900 \\
1315 \\
1330 \\
1410\end{array}$ & $\begin{array}{l}583 \\
470 \\
530 \\
520 \\
510 \\
334\end{array}$ & $\begin{array}{c}2.4 \\
.38 \\
.28 \\
2.3 \\
2.2 \\
9.3\end{array}$ & $\begin{array}{r}6 \\
54 \\
62 \\
56 \\
60 \\
104\end{array}$ & $\begin{array}{l}.04 \\
.06 \\
.05 \\
.35 \\
.36 \\
2.6\end{array}$ & $\begin{array}{c}.54 \\
.09 \\
.06 \\
.52 \\
.50 \\
2.1\end{array}$ \\
\hline
\end{tabular}

WAIER YEAR OCTOBER 1984 TO SEPTEMBER 1985

\begin{tabular}{|c|c|c|c|c|c|c|c|}
\hline$O C I$ & 25 & 1400 & 227 & 9 & 93 & 2.3 & 2.0 \\
\hline $\mathrm{OCT}$ & 29 & 1325 & & 3.1 & 96 & .80 & .70 \\
\hline NOV & 5 & 1630 & 75 & 561 & 1,640 & 2,480 & 28 \\
\hline NOV & 7 & 1515 & 220 & 44 & 141 & 17 & 10 \\
\hline NOV & 7 & 1600 & 222 & 46 & 159 & 20 & 10 \\
\hline NOV & 8 & $150 \mathrm{~B}$ & 403 & 6.7 & 70 & 1.3 & 1.5 \\
\hline NOV & 8 & 1547 & 407 & 6.6 & 95 & 1.7 & 1.5 \\
\hline NOV & 14 & 1425 & 595 & 2.4 & 87 & .60 & .54 \\
\hline NOV & 20 & 1555 & 620 & 2 & 9 & .05 & .45 \\
\hline$D E C$ & 5 & 1305 & 620 & 1.6 & 3 & .01 & .36 \\
\hline$D E C$ & 19 & 1346 & 630 & 1.3 & 66 & .24 & .30 \\
\hline JAN & 11 & 1311 & 660 & 1.1 & 86 & .26 & .25 \\
\hline JAN & 21 & 1200 & 730 & .79 & 83 & .18 & $.1 \mathrm{~B}$ \\
\hline FEB & 14 & 1110 & 695 & .54 & 7 & .01 & .12 \\
\hline MAR & 11 & 1200 & 660 & .89 & 2 & .01 & .20 \\
\hline APH & 15 & 1207 & 650 & .58 & 4 & .01 & .13 \\
\hline MAY & 15 & 1830 & 108 & 254 & 676 & 464 & 58 \\
\hline MAY & 15 & 1845 & 108 & 191 & 501 & 258 & 43 \\
\hline MAY & 15 & 1900 & 107 & 156 & 403 & 170 & 35 \\
\hline MAY & 15 & 1915 & 110 & 129 & 314 & 109 & 29 \\
\hline MAY & 15 & 1930 & 111 & 112 & 316 & 96 & 25 \\
\hline MAY & 15 & 1945 & 112 & 104 & 252 & 71 & 24 \\
\hline MAY & 15 & 2000 & 126 & 102 & 226 & 63 & 23 \\
\hline MAY & 16 & 1410 & 318 & 6.4 & 32 & .55 & 1.4 \\
\hline MAY & 17 & 1520 & 128 & 65 & 402 & 70 & 15 \\
\hline MAY & 17 & 1525 & 120 & 63 & 372 & 63 & 14 \\
\hline MAY & 18 & 1655 & 106 & 185 & 408 & 203 & 42 \\
\hline MAY & 21 & 1010 & 480 & 2.1 & 13 & .07 & .48 \\
\hline MAY & 29 & 1355 & 580 & .89 & 11 & .26 & .20 \\
\hline JUNit & 6 & 1120 & 564 & .79 & 12 & .26 & .18 \\
\hline JUNE & 12 & 1405 & 585 & .66 & 10 & .02 & .15 \\
\hline JUNE & 24 & 1300 & 540 & .66 & 11 & .02 & .15 \\
\hline JULY & 8 & 1345 & 540 & .54 & 28 & .04 & .12 \\
\hline JULY & 22 & 1425 & 495 & .89 & 45 & .06 & .20 \\
\hline AUG & 1 & 1325 & 595 & .75 & 9 & .02 & .17 \\
\hline AUG & 16 & 1225 & 550 & .45 & 5 & .01 & .10 \\
\hline AUG & 30 & 1440 & 550 & .51 & 7 & .01 & .12 \\
\hline SEPI & 9 & 1455 & 485 & .84 & 8 & .02 & .19 \\
\hline SEPI & 13 & 1423 & 180 & -- & 114 & $\cdots$ & $\cdots$ \\
\hline SEPI & 27 & 1500 & 390 & 4.9 & 137 & 1.8 & 1.1 \\
\hline
\end{tabular}

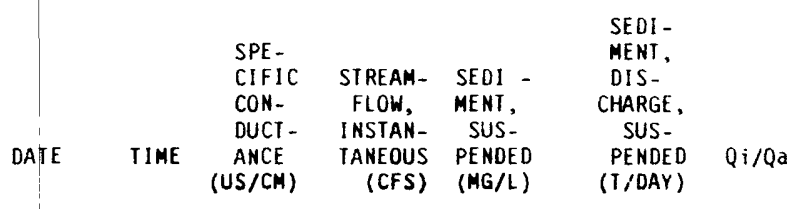

WATER YEAR OCTOBER 1984 TO SEPTEMBER $19 B 5$

$\begin{array}{lllllll}\text { SEPI } 27 & 1505 & 370 & 5.5 & 173 & 2.6 & 1.2 \\ \text { SEPI } 27 & 1515 & 310 & 6.7 & 227 & 4.1 & 1.5 \\ \text { SEPT 27 } & 1520 & 310 & 6.1 & 234 & 3.9 & 1.4\end{array}$

\section{WATER YEAR OCTOBER 1985 TO SEPTEMBER 1986}

\begin{tabular}{l|rrrrrrr} 
OCT & 6 & 1430 & 90 & 318 & 392 & 337 & 72 \\
OCT & 6 & 1445 & 80 & 339 & 445 & 407 & 77 \\
OCT & 6 & 1500 & 90 & 380 & 498 & 511 & B6 \\
OCT & 6 & 1510 & 80 & 490 & 707 & 935 & 111 \\
OCT & 6 & 1515 & 90 & 545 & 965 & 1,420 & 124 \\
& & & & & & & \\
OCT & 6 & 1515 & 80 & 545 & 968 & 1,420 & 124 \\
OCT & 6 & 1520 & 70 & 569 & 1,550 & 2,380 & 129 \\
OCT & 6 & 1525 & 70 & 593 & 1,360 & 2,180 & 135 \\
OCT & 6 & 1530 & 80 & 617 & 1,290 & 2,150 & 140 \\
OCT & 6 & 1535 & 80 & 604 & 1,360 & 2,220 & 137 \\
& & & & & & & \\
OCT & 6 & 1540 & 80 & 591 & 1,280 & 2,040 & 134 \\
OCT & 6 & 1545 & 80 & 578 & 1,160 & 1,810 & 131 \\
OCT & 6 & 1550 & 80 & 583 & 1,280 & 2,010 & 132 \\
OCT & 6 & 1555 & 80 & 588 & 1,280 & 2,010 & 134 \\
OCT & 6 & 1600 & 80 & 592 & 1,320 & 2,110 & 134 \\
& & & & & & & \\
OCT & 6 & 1605 & 80 & 559 & 1,300 & 1,960 & 127 \\
OCT & 6 & 1615 & 70 & 494 & 1,250 & 1,670 & 112 \\
OCT & 6 & 1630 & 70 & 412 & 1,030 & 1,150 & 94 \\
OCT & 6 & 1645 & 80 & 337 & 900 & 819 & 77 \\
OCT & 6 & 1700 & 90 & 302 & 798 & 651 & 69 \\
& & & & & & &
\end{tabular}

$\begin{array}{llllllll}\text { OCT } & 7 & 1200 & 160 & 58 & 162 & 25 & 13 \\ \text { OCT } & 7 & 1215 & 160 & 57 & 137 & 21 & 13 \\ \text { OCT } & 7 & 1230 & 160 & 57 & 137 & 21 & 13 \\ \text { OCT } & 7 & 1245 & 160 & 57 & 142 & 22 & 13\end{array}$

$\begin{array}{llllllll}\mathrm{OCT} & 7 & 1245 & 160 & 57 & 142 & 22 & 13\end{array}$

$\begin{array}{llllllll}\text { OCT } & 7 & 1315 & 170 & 53 & 122 & 17 & 12\end{array}$

\begin{tabular}{l|lllllrr}
$O C T$ & 24 & 0845 & 260 & 16 & 324 & 14 & 3.6 \\
$O C T$ & 24 & 0850 & 260 & 18 & 105 & 5.1 & 4.1
\end{tabular}

\begin{tabular}{l|rrrrrrr}
$O C T$ & 24 & 0850 & 260 & 18 & 105 & 5.1 & 4.1 \\
$O C T$ & 24 & 0900 & 260 & 21 & 99 & 5.6 & 4.8
\end{tabular}

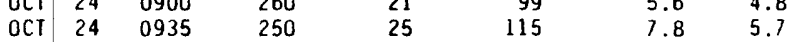

$\begin{array}{llllllll}\text { OCT } & 29 & 0810 & 180 & 71 & 341 & 65 & 16\end{array}$

\begin{tabular}{l|lllllll} 
OCI & 29 & 0818 & 210 & 71 & 341 & 65 & 16 \\
OCI & 29 & 0825 & 190 & 87 & 356 & 72 & 17 \\
\hline
\end{tabular}

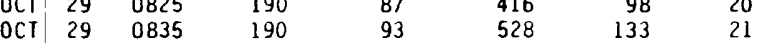

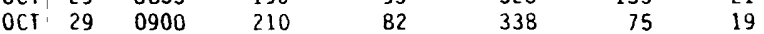

\begin{tabular}{l|lllllll} 
OCT & 29 & 0930 & 230 & 72 & 501 & 97 & 16
\end{tabular}

\begin{tabular}{l|rrrrrrr} 
NOV & 14 & 0925 & 460 & 3.2 & 14 & .12 & .73 \\
NOV & 27 & 0940 & 510 & 2.8 & 9 & .07 & .64
\end{tabular}

\begin{tabular}{l|rrrrrrr} 
NOV & 27 & 0940 & 510 & 2.8 & 9 & .07 & .64 \\
DEC & 6 & 1340 & 560 & 2.4 & 27 & .17 & .54 \\
DEC & 12 & 0925 & 575 & 2.4 & 4 & .03 & .54
\end{tabular}

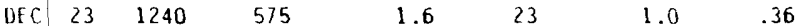

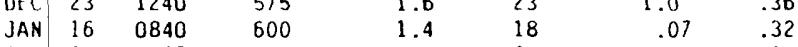

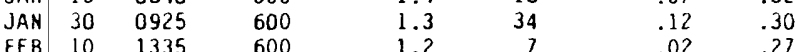

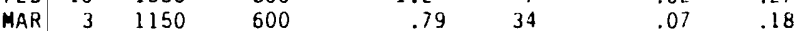

$\begin{array}{llllllll}\text { MAR } & 20 & 1020 & 600 & .76 & 53 & .11 & .17\end{array}$

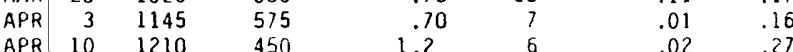

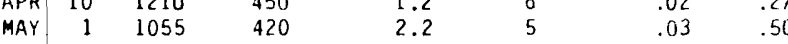

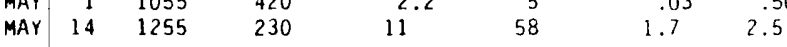

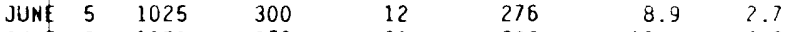

JUNE $\begin{array}{lllllll}9 & 1150 & 350 & 21 & 228 & 13 & 4.8\end{array}$

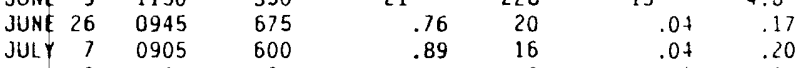

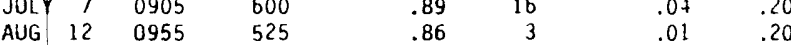

AUG $26 \quad 0940 \quad 675$

SEPT $15 \quad 0955 \quad 600$

$\begin{array}{rr}.70 & 28 \\ .57 & 9\end{array}$

$.05 \quad .16$ 


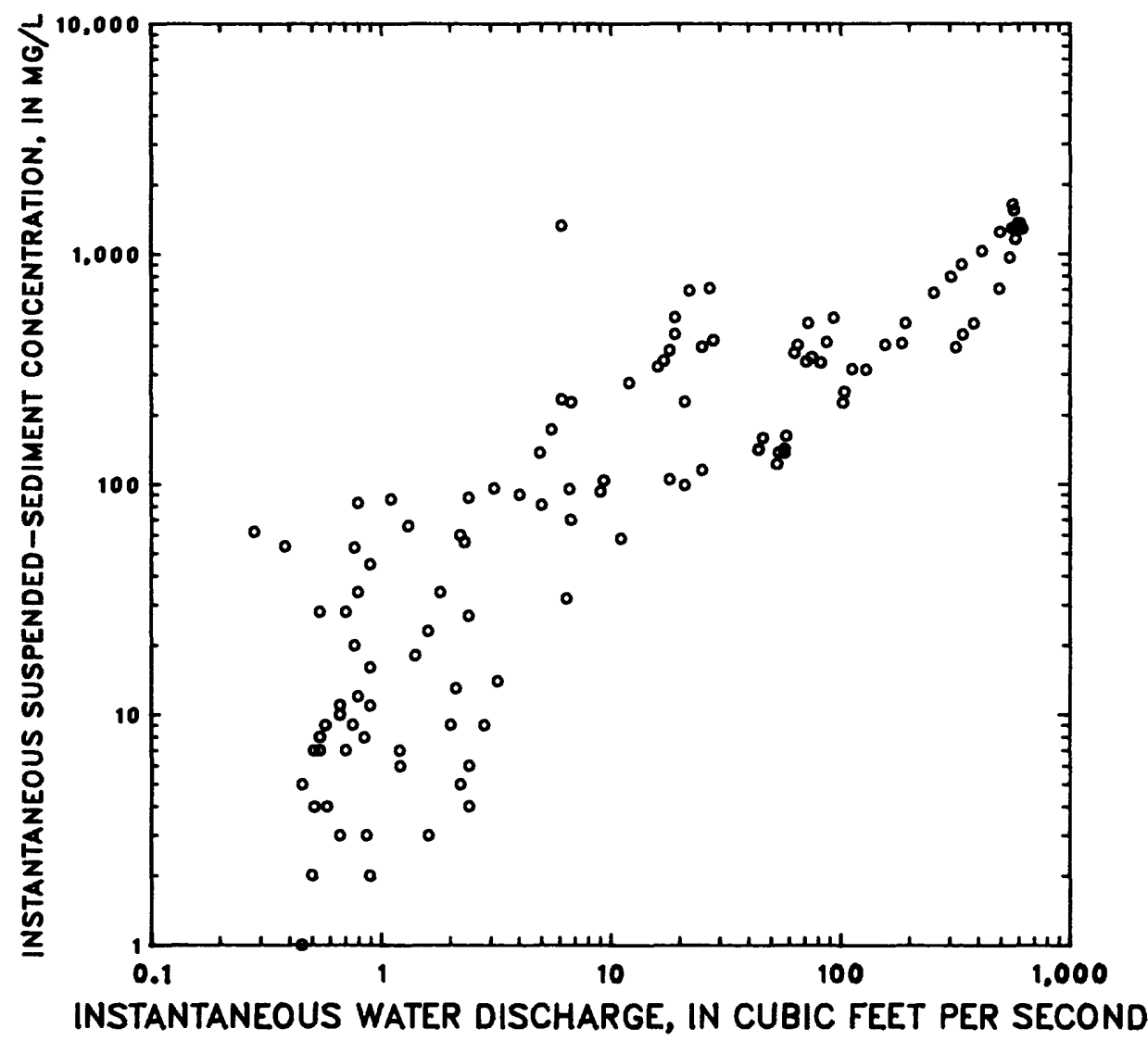

Figure 12.--Relation of suspended-sediment concentration to stream discharge for station Quebrada Mamey near Gurabo. 
Table 13. Suspended-sediment data for samples collected at station Rio Gurabo at Gurabo

[US/CM, microsiemens per centimeter; CFS, Cubic foot per second; MG/L, milligrams per liter; T/DAY, tons per day: Qi/Qa, ratio of instantaneous water discharge to average water discharge; ...., missing data]

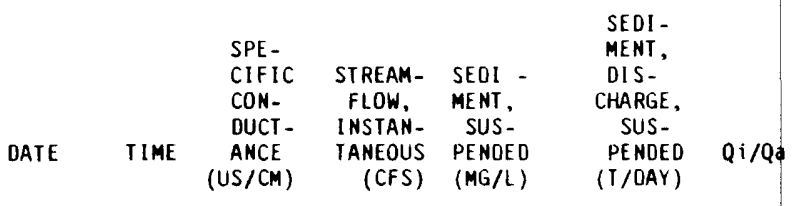

WATER YEAR OCTOBER 1983 TO SEPTEMBER 1984

$\begin{array}{lrrrrr}\text { FEB } & 21 & 1440 & 355 & 39 & 40 \\ \text { MAR } & 12 & 1200 & 382 & 25 & 3 \\ \text { MAR } & 26 & 1230 & 377 & 19 & 8 \\ \text { APR } & 10 & 1245 & 418 & 25 & \\ \text { APR } & 26 & 1230 & 390 & 14 & 42 \\ & & & & & \\ \text { MAY } & 16 & 1330 & 391 & 20 & 10 \\ \text { JULY } 5 & 1424 & 188 & 332 & 268 \\ \text { JULY } & 5 & 1426 & 188 & 332 & 26 \\ \text { SEPT } 4 & 1200 & 270 & 66 & 109 \\ \text { SEPT } 19 & 1500 & --- & 269 & 179\end{array}$

$\begin{array}{rcr}40 & 4.2 & 0.29 \\ 3 & .20 & .19 \\ 8 & .41 & .14 \\ 9 & .61 & .18 \\ 42 & 1.6 & .19 \\ 10 & & .15 \\ 268 & 240 & 2.5 \\ 264 & 237 & 2.5 \\ 109 & 19 & .49 \\ 179 & 130 & 2.0\end{array}$

WATER YEAR OCTOBER 1984 TO SEPTEMBER 1985

\begin{tabular}{|c|c|c|c|c|c|c|c|}
\hline$O C I$ & 25 & 1345 & $--\cdot$ & 156 & 105 & 44 & 1.2 \\
\hline $\mathrm{OCT}$ & 25 & 1430 & 252 & 154 & 156 & 65 & 1.1 \\
\hline HOV & 5 & 1730 & 85 & 5,950 & 1,710 & 27.500 & +4 \\
\hline NOV & 8 & 1020 & 204 & 472 & 253 & 322 & 3.5 \\
\hline NOV & 8 & 1330 & 204 & 433 & 189 & 221 & 3.2 \\
\hline NOV & 14 & 1440 & 320 & 206 & 50 & 28 & 1.5 \\
\hline NOV & 20 & 1520 & 335 & 147 & 43 & 17 & 1.1 \\
\hline UEC & 5 & 1340 & 336 & 73 & $5 ?$ & 11 & .54 \\
\hline DEC & 19 & 1423 & 338 & 55 & 62 & 9.2 & .41 \\
\hline JAN & 11 & 1359 & 370 & 52 & 66 & 9.3 & .38 \\
\hline JAN & 23 & 1130 & 387 & 31 & 100 & 8.4 & .23 \\
\hline FEB & 14 & 1140 & 412 & 21 & 46 & 2.6 & .16 \\
\hline MAR & 11 & 1230 & 346 & 34 & 57 & 5.2 & .25 \\
\hline APR & 15 & 1230 & 360 & 40 & 11 & 1.2 & .30 \\
\hline MAY & 15 & 1035 & 118 & 1,850 & 1.410 & 7.040 & 14 \\
\hline MAY & 15 & 1100 & 120 & 2,160 & 1,400 & 8.160 & 16 \\
\hline MAY & 15 & 1115 & 117 & 2,490 & 1,230 & 8,270 & 18 \\
\hline MAY & 15 & 1130 & 112 & 2,920 & 1,660 & 13,100 & 22 \\
\hline MAY & 15 & 1145 & 110 & 3,410 & 1,770 & 16,300 & 25 \\
\hline MAY & 15 & 1200 & 101 & 3,920 & 2.010 & 21.300 & 29 \\
\hline MAY & 15 & 1215 & 100 & 4,440 & 3.010 & 36,100 & 33 \\
\hline MAY & 15 & 1300 & 82 & 6,040 & 2,600 & 42,400 & 45 \\
\hline MAY & 15 & 1315 & 82 & 6,500 & 2,020 & 35,500 & 48 \\
\hline MAY & 15 & 1330 & 78 & 6.850 & 1,630 & 30,100 & 51 \\
\hline MAY & 15 & 1400 & 75 & 8.260 & 1.960 & 43,700 & 61 \\
\hline MAY & 15 & 1430 & 83 & 11,900 & 2,500 & 80,300 & 88 \\
\hline MAY & 21 & 0925 & 242 & 162 & 70 & 31 & 1.2 \\
\hline MAY & 29 & 1310 & 279 & 61 & 9 & 1.5 & .45 \\
\hline JUNE & 5 & 1245 & 275 & 37 & 24 & 2.4 & .27 \\
\hline JUNE & 12 & 1305 & 288 & 42 & 20 & $\angle . j$ & 0.31 \\
\hline JUNE & 20 & 1325 & 306 & 42 & 109 & 12 & .31 \\
\hline JUNE & 28 & 0910 & 310 & 24 & 14 & .91 & .18 \\
\hline JULYY & 5 & 0905 & 282 & 21 & 54 & 3.1 & .16 \\
\hline JULY & 16 & 1335 & $\cdots$ & 301 & 575 & 407 & 2.2 \\
\hline JULY & 25 & 0900 & 275 & 52 & 18 & 2.5 & .38 \\
\hline AUG & 9 & 1315 & 275 & 26 & 8 & .58 & $.1 y$ \\
\hline AUG & 16 & 1155 & 280 & 27 & 10 & .73 & .20 \\
\hline AUG & 23 & 0920 & 280 & 30 & 34 & 2.6 & .22 \\
\hline SEPT & 3 & 1410 & 320 & 34 & 16 & 1.5 & .25 \\
\hline
\end{tabular}

\section{WATER YEAR OCTOBER 1985 TO SEPTEMBER $1986^{\circ}$}

\begin{tabular}{lrrrrrrr|} 
OCT & 3 & 0840 & 210 & 202 & 560 & 305 & 1.5 \\
OCT & 3 & 0900 & 210 & 237 & 1520 & 973 & 1.8 \\
OCT & 3 & 0910 & 210 & 257 & 146 & 101 & 1.9 \\
OCT & 3 & 0930 & 210 & 293 & 170 & 134 & 2.2 \\
OCT & 3 & 0945 & 210 & 302 & 100 & 82 & 2.2
\end{tabular}

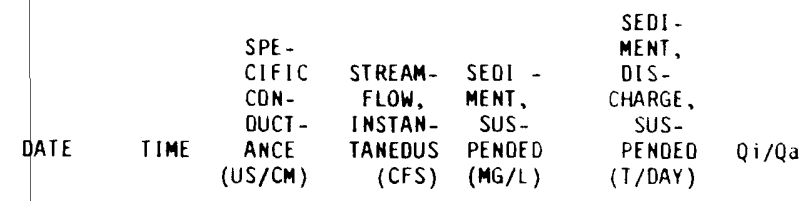

WATER YEAR OCTOBER 1985 TO SEPTEMBER 1986

$\begin{array}{lllllllr}\text { OCI } & 6 & 1300 & 65 & 11,500 & 1,390 & 43,200 & 85 \\ \text { OCT } & 6 & 1315 & 70 & 11,600 & 1,390 & 43,500 & 86 \\ \text { OCT } & 6 & 1330 & 65 & 11,800 & 1,570 & 50,000 & 87 \\ \text { OCT } & 6 & 1345 & 65 & 11,900 & 1,360 & 43,700 & 88 \\ \text { OCT } & 6 & 1400 & 70 & 11,800 & 1,550 & 49,400 & 87 \\ & & & & & & & \\ \text { OCT } & 6 & 1415 & 70 & 11,600 & 1,560 & 48,900 & 86 \\ \text { OCT } & 6 & 1430 & 70 & 11,600 & 1,390 & 43,500 & 86 \\ \text { OCT } & 6 & 1500 & 70 & 11,900 & 1,170 & 37,600 & 88 \\ \text { OCT } & 6 & 1530 & 60 & 12,600 & 1,360 & 46,300 & 93 \\ \text { OCT } & 6 & 1630 & 70 & 14,500 & 1,460 & 57,200 & 107 \\ \text { OCI } & 6 & 1700 & 60 & 15,600 & 1,420 & 59,800 & 115 \\ \text { OCI } & 6 & 1715 & 60 & 16,100 & 1,310 & 56,900 & 119 \\ \text { OCT } & 6 & 1730 & 60 & 16,600 & 1,320 & 59,200 & 123 \\ \text { OCT } & 6 & 1745 & 60 & 16,900 & 1,280 & 58,400 & 125 \\ \text { OCT } & 6 & 1800 & 60 & 17,200 & 1,270 & 59,000 & 127\end{array}$

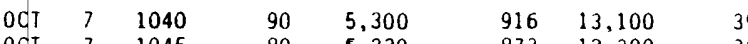

OCT $7 \quad 1045$

OCT 71050

OCI 7 1100

OCI $7 \quad 1105$

$\begin{array}{lll}0 \mathrm{CI} ? & 7110 \\ 0 \mathrm{CI} & 7 & 1115\end{array}$

od 7 1120

OCT 71125

OCT 71130

$\begin{array}{lll}\text { OCT } & 7 & 1135 \\ 0 \mathrm{CT} & 7 & 1140\end{array}$

OCT 71145

ocr 71150

OCT 71155

OCT 71200

OCT 71205

$\begin{array}{lll}\text { OCT } & 7 & 1210 \\ \text { OCT } & 7 & 1215\end{array}$

OCT $7 \quad 1220$

$\begin{array}{lll}\text { OCT } & 7 & 1225 \\ \text { OCT } & 7 & 1230\end{array}$

$\begin{array}{lll}\text { OCT } & 7 & 1230 \\ \text { OCT } & 7 & 1235\end{array}$

OCT $7 \quad 1240$

$\begin{array}{lll}\text { OCT } & 7 & 1245 \\ \text { OCT } & 7 & 1250\end{array}$

OCT 71255

$\begin{array}{lll}\text { OCT } & 7 & 1300 \\ \text { OCT } & 7 & 1305\end{array}$

OCT 71310

$\begin{array}{lll}\text { OCT } & 7 & 1315 \\ \text { OCT } & 7 & 1320\end{array}$

$\begin{array}{lll}\text { OCT } & 7 & 1320 \\ \text { OCT } & 7 & 1325\end{array}$

OCT $7 \quad 1330$

OCI 71335

$\begin{array}{lll}\text { OCT } & 7 & 1340 \\ \text { OCT } & 1345\end{array}$

OCT 71350

$849 \quad 6.460$
5,230

5,140

$873 \quad 12,300$

90212,300

$\begin{array}{lll}4,990 & 892 & 12,000\end{array}$

4,910

86011,400

4,820

4,740

4,670

$867 \quad 11,300$

$849 \quad 10,900$

4,610

$876 \quad 10,900$

4,540

4,470

4,390

4,320

$980 \quad 12,000$

$\begin{array}{ll}872 & 10,500 \\ 895 & 10,600\end{array}$

$895 \quad 10.600$

$858 \quad 9,960$

4,270

4,090

$826 \quad 9.520$

$902 \quad 9,960$

$867 \quad 9,390$

4,010

3,940

$867 \quad 9.220$

8889,250

3,830

3,800

3,700

3,640

$938 \quad 9,690$

$942 \quad 9,660$

$938 \quad 9,370$

$916 \quad 9,000$

$900 \quad 8,660$

903,480

8,140

$906 \quad 8,340$

$840 \quad 7,550$

$980 \quad 8.620$

8737,540

3,330

3,260

3,200

916

7,770

3,140

2,970

955

$910 \quad 7.940$

873

$\begin{array}{ll}879 & 7,000\end{array}$

$876 \quad 0.700$

8816,590

$794 \quad 5,830$

$793 \quad 5,740$

86
87

$8 t$

88

93
107

115

119

125

39

39
38

38
37

36

36

35
34

34

33
32

32
32

32

32
30
30

30

29
28

28

28

27
27

26

26

25
25

25

24

23

23
23

22

22

21

$\begin{array}{ll}100 & 2,820 \\ 100 & 2,770\end{array}$

$100 \quad 2,720$

2,680 
Table 13. Suspended-sediment data for samples collected at station Rio Gurabo at Gurabo

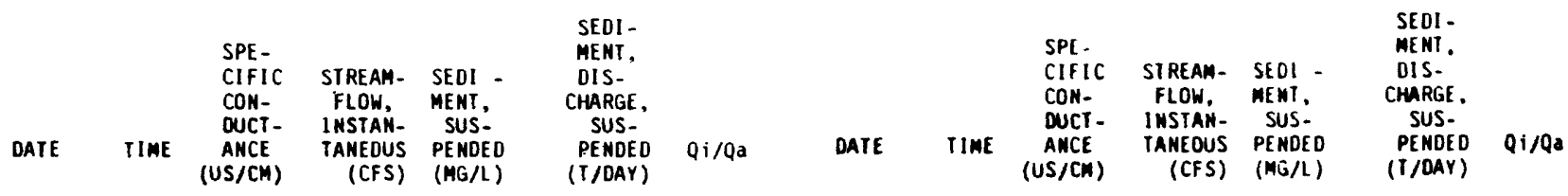

WAIER YEAR OCTOBER 198510 .SEPIEMBER 1986-Cont inued

$\begin{array}{lrrrr}\text { OCT } & 18 & 0930 & 280 & 130 \\ \text { OCT } & 31 & 1235 & 170 & 302 \\ \text { NOV } & 14 & 0855 & 200 & 178 \\ \text { NOV } & 27 & 0850 & 350 & 140 \\ \text { DEC } & 12 & 0905 & 350 & 67 \\ & & & & \\ \text { DEC } & 19 & 1010 & 360 & 59 \\ \text { DEC } & 27 & 0 B 40 & 360 & 48 \\ \text { JAN } & 16 & 0815 & 400 & 31 \\ \text { JAN } & 23 & 0850 & 380 & 33 \\ \text { JAN } & 30 & 0855 & 375 & 30 \\ & & & & \\ \text { FEB } & 13 & 0945 & 400 & 25 \\ \text { FEB } & 24 & 0810 & 380 & 23 \\ \text { MAR } & 20 & 1050 & 380 & 15 \\ \text { APR } & 3 & 1220 & 320 & 36 \\ \text { APR } & 10 & 1245 & 300 & 43\end{array}$

$\begin{array}{rcc}31 & 11 & 7.0 \\ 347 & 283 & 2.2 \\ 85 & 41 & 1.3 \\ 11 & 4.2 & 1.0 \\ 10 & 1.8 & .50 \\ & & \\ 14 & 2.2 & .43 \\ 14 & 1.8 & .36 \\ 32 & 2.7 & .23 \\ 11 & .98 & .24 \\ 8 & .65 & .22 \\ & & \\ 39 & 2.6 & .18 \\ 13 & .81 & .17 \\ 12 & .49 & .11 \\ 22 & 2.1 & .27 \\ 42 & 4.9 & .32\end{array}$

$\begin{array}{lrrr}\text { APR } & 17 & 1120 & 390 \\ \text { MAY } & 8 & 1320 & 250 \\ \text { MAY } & 14 & 1215 & 120 \\ \text { MAY } & 14 & 1230 & 120 \\ \text { JUNE } & 9 & 1210 & 200 \\ & & & \\ \text { JUNE } & 9 & 1220 & 190 \\ \text { JUNE } & 9 & 1230 & 180 \\ \text { JUNE } & 9 & 1245 & 180 \\ \text { JUNE } & 26 & 0900 & 360 \\ \text { JULY } & 7 & 0840 & 220 \\ & & & \\ \text { AUG } & 10 & 0930 & 280 \\ \text { AUG } & 26 & 0915 & 380 \\ \text { SEPT } & 2 & 1325 & 300 \\ \text { SEPT } & 15 & 0925 & 340\end{array}$

21
42
965
926
993
980
961
926
12
13
20
11
15
9.4

12
50
378
438

$\begin{array}{rl}5.68 & .16 \\ 985 & 7.27 \\ 1,100 & 6.9 \\ 3,790 & 7.4\end{array}$

1,180

$3,110 \quad 7.3$

$1,140 \quad 2,960 \quad 7.1$

995

6.9

$\begin{array}{ll}.87 & .09 \\ 5.1 & .10\end{array}$

$\begin{array}{ll}2.3 & .15 \\ 0 & .08 \\ 6.7 & .11\end{array}$

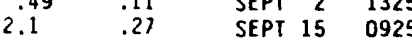

.13

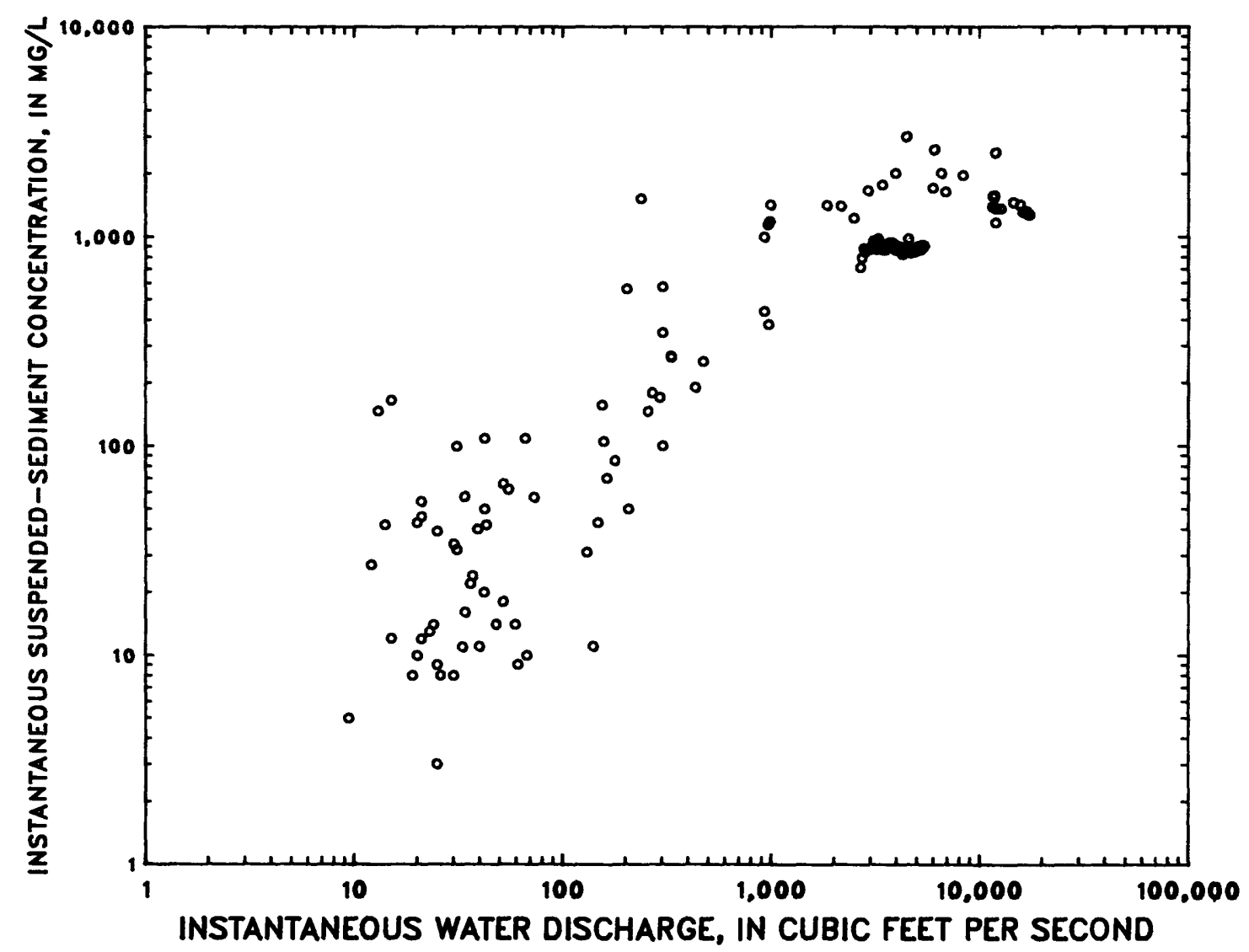

Figure 13.--Relation of suspended-sediment concentration to stream discharge for station Río Gurabo at Gurabo. 
Table 14. Summary of equations, discharge ranges, and number of suspended-sediment samples used for pump sampler data

[DY, shift (amount positive o negative) ${ }_{3}$ to be added to old sediment load value; $Q$, water discharge; $\mathrm{ft}^{3} / \mathrm{s}$, cubic feet per second; $>$, greater than; $\leq$, less or equal than; $C L$, calibrated or new sediment load value; L, old sediment load value; CAL, percent calibration (positive or negative) from old; CS, calibrated or new sediment concentration value; 0.0027 , conversion factor]

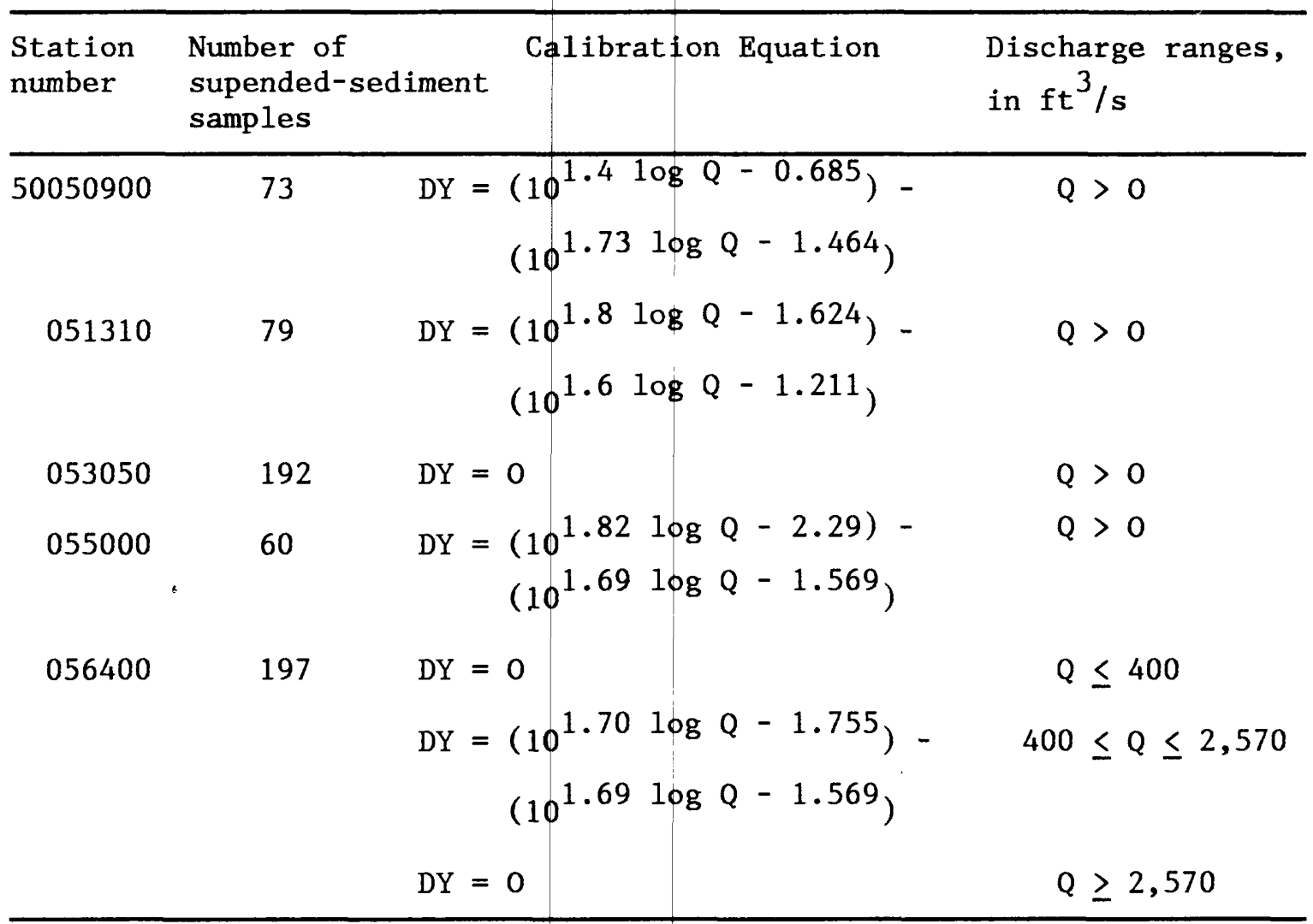

For all calibrated or new values:

$$
\mathrm{CL}=\mathrm{L}+\mathrm{DY} \quad \mathrm{CAL}=\left(\frac{\mathrm{L}-\mathrm{CL}}{\mathrm{L}}\right) 100 \quad \mathrm{CS}=\frac{\mathrm{CL}}{0.0027 \mathrm{Q}}
$$




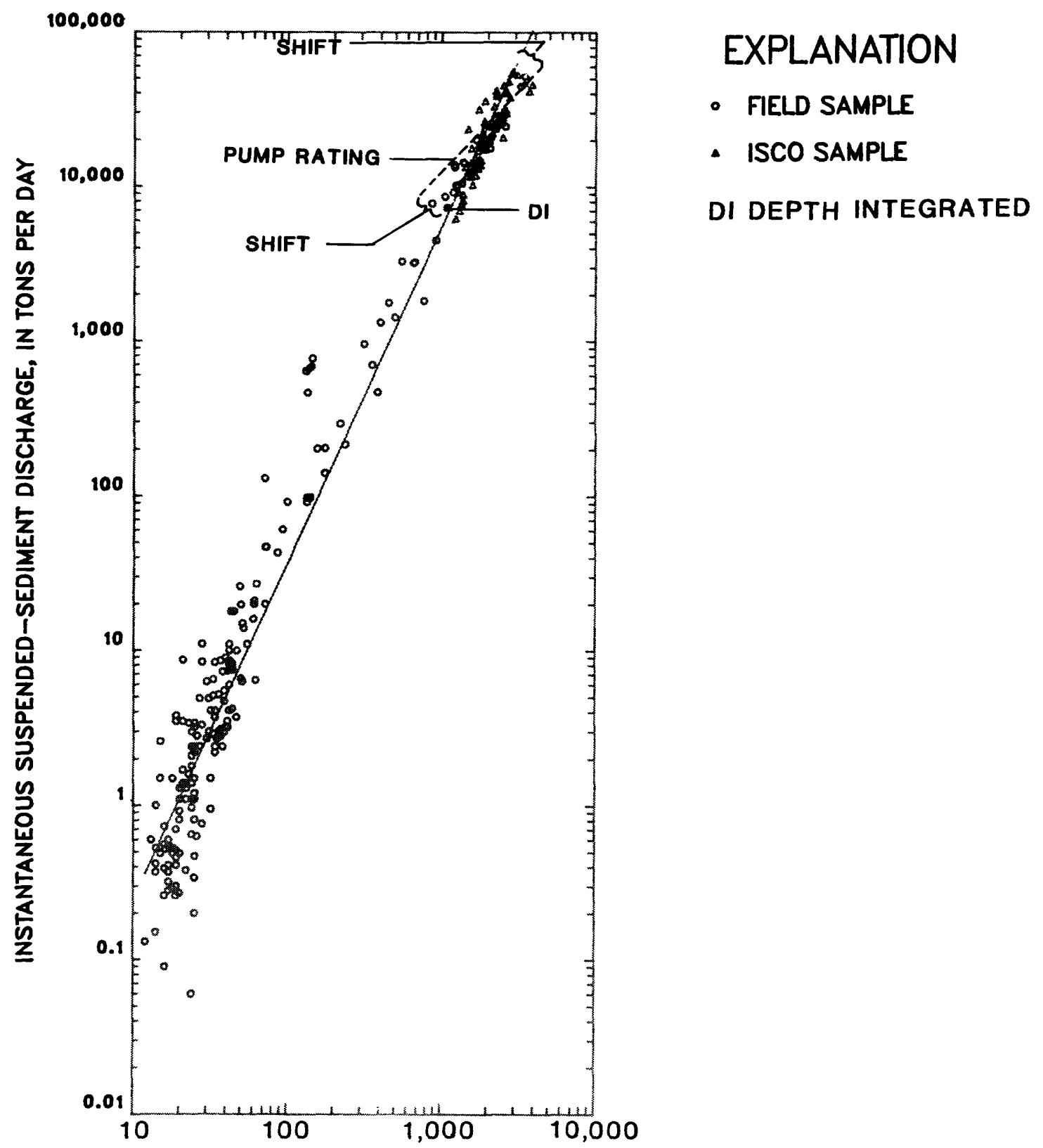

INSTANTANEOUS WATER DISCHARGE, IN CUBIC FEET PER SECOND

Figure 14,--Idealized plot of calibration procedure for pump-sampler data 
Table 15. Particle-size distribution data from suspended-sediment samples collected at ten surface-water stations in Rio Grande de Loiza basin [CFS, cubic feet per second; MG/L, milligrams per liter; T/DAY, tons per day; MM, millimeters; ---, missing data; e, est imated]

\begin{tabular}{|c|c|c|c|c|c|c|c|c|c|c|c|c|c|c|}
\hline Date & T ine & $\begin{array}{l}\text { Strean } \\
\text { Flow } \\
\text { Instan- } \\
\text { taneous } \\
\text { (CFS) }\end{array}$ & $\begin{array}{l}\text { Sed. } \\
\text { Susp. } \\
\text { Conc. } \\
\text { (MG/L) }\end{array}$ & $\begin{array}{l}\text { Sed. } \\
\text { Susp. } \\
\text { Dis- } \\
\text { charge } \\
\text { (T/DAY) }\end{array}$ & $\begin{array}{l}\text { Sed. } \\
\text { Susp. } \\
\text { Fall } \\
\text { Diam. } \\
\% \text { Finer } \\
\text { Than } \\
.002 \text { MM }\end{array}$ & $\begin{array}{l}\text { Sed. } \\
\text { Susp. } \\
\text { Fail } \\
\text { Diam. } \\
\% \text { Finer } \\
\text { Than } \\
.004 \text { MM }\end{array}$ & $\begin{array}{l}\text { Sed. } \\
\text { Susp. } \\
\text { Fall } \\
\text { Diam. } \\
\text { * Finer } \\
\text { Than } \\
.008 \text { mM }\end{array}$ & $\begin{array}{l}\text { Sed: } \\
\text { Susp. } \\
\text { Fall } \\
\text { Dian. } \\
\text { \% Finer } \\
\text { Than } \\
.016 \text { Mm }\end{array}$ & $\begin{array}{l}\text { Sed. } \\
\text { Susp. } \\
\text { Fall } \\
\text { Diam. } \\
\% \text { Finer } \\
\text { Than } \\
.031 \text { MM }\end{array}$ & $\begin{array}{l}\text { Sed. } \\
\text { Susp. } \\
\text { Sieve } \\
\text { Diam. } \\
\text { \% Finer } \\
\text { Than } \\
.062 \text { MM }\end{array}$ & $\begin{array}{l}\text { Sed. } \\
\text { Susp. } \\
\text { Sieve } \\
\text { Diam. } \\
\text { \% Finer } \\
\text { Than } \\
.125\end{array}$ & $\begin{array}{l}\text { Sed. } \\
\text { Susp. } \\
\text { Sieve } \\
\text { Diam. } \\
\% \text { Finer } \\
\text { Than } \\
.250 \text { Mm }\end{array}$ & $\begin{array}{l}\text { Sed. } \\
\text { Susp. } \\
\text { Sieve } \\
\text { Diam. } \\
\% \text { Finer } \\
\text { Than } \\
.500 \text { MM }\end{array}$ & $\begin{array}{l}\text { Seu. } \\
\text { Susp. } \\
\text { Sieve } \\
\text { Diam. } \\
\% \text { Finer } \\
\text { Than } \\
1.00 \text { mm }\end{array}$ \\
\hline
\end{tabular}

50050900 Rio Grande de Loiza at Quebrada Arenas

Water rear October 1984 to September 1985

\begin{tabular}{|c|c|c|c|c|c|c|c|c|c|c|c|c|c|c|c|}
\hline SEPI & 25 & 0050 & 1.890 & 3.600 & 18,400 & $t$ & 8 & 14 & 25 & 37 & to & 66 & 79 & 87 & 94 \\
\hline & & & & & & ater & $0<t$ & 1985 & epte & 1986 & & & & & \\
\hline \multirow{6}{*}{$\begin{array}{l}O C T \\
O C T \\
O C I \\
O C I \\
O C T\end{array}$} & 6 & 0745 & 1.260 & 2,190 & 7,440 & 4 & 6 & 15 & 26 & 33 & $3 t$ & 54 & 66 & 78 & 87 \\
\hline & 6 & 1000 & 2.990 & 4,200 & 33,900 & 6 & 9 & 17 & 28 & 39 & 51 & 67 & 86 & 94 & 98 \\
\hline & 6 & 1030 & 2,910 & 2,280 & 17,900 & 8 & 14 & 24 & 35 & 51 & 62 & 86 & 90 & 97 & 98 \\
\hline & 26 & 1620 & 865 & 7.510 & 17,500 & 7 & 13 & 20 & 30 & 41 & 58 & 78 & 93 & 97 & 99 \\
\hline & 26 & 1635 & 1.040 & 4.430 & 12,400 & 10 & 15 & 24 & 37 & 51 & 60 & 74 & 84 & 90 & 99 \\
\hline & & & & & Average & 7 & 11 & 19 & 30 & $+\angle$ & $52^{\circ}$ & 71 & 83 & 90 & 96 \\
\hline
\end{tabular}

50051150 Quebrada blanca at Jagual

Water Year October 1983 to September 1984

\begin{tabular}{|c|c|c|c|c|c|c|c|c|c|c|c|c|c|c|c|}
\hline $\begin{array}{l}\text { SEPT } \\
\text { SEPT } \\
\text { SEPT } \\
\text { SEPT } \\
\text { SEPT }\end{array}$ & $\begin{array}{r}5 \\
5 \\
5 \\
5 \\
13\end{array}$ & $\begin{array}{l}1500 \\
1503 \\
1510 \\
1515 \\
1435\end{array}$ & $\begin{array}{c}\text { e } 100 \\
\text { el00 } \\
\text { e95 } \\
\text { e95 } \\
83\end{array}$ & $\begin{array}{l}50,400 \\
43,100 \\
37,500 \\
37,100 \\
40,100\end{array}$ & $\begin{array}{r}13,600 \\
11,600 \\
9,620 \\
9.500 \\
8,990\end{array}$ & $\begin{array}{l}30 \\
25 \\
33 \\
30 \\
26\end{array}$ & $\begin{array}{l}+1 \\
+3 \\
+4 \\
+2 \\
35\end{array}$ & $\begin{array}{l}54 \\
56 \\
57 \\
56 \\
47\end{array}$ & $\begin{array}{l}71 \\
74 \\
73 \\
72 \\
61\end{array}$ & $\begin{array}{l}83 \\
87 \\
85 \\
85 \\
77\end{array}$ & $\begin{array}{l}89 \\
92 \\
92 \\
90 \\
84\end{array}$ & $\begin{array}{l}96 \\
97 \\
97 \\
97 \\
95\end{array}$ & $\begin{array}{l}97 \\
99 \\
99 \\
99 \\
99\end{array}$ & $\begin{array}{r}97 \\
100 \\
100 \\
100 \\
100\end{array}$ & $\begin{array}{r}98 \\
100 \\
100 \\
100 \\
100\end{array}$ \\
\hline $\begin{array}{l}\text { SEPT } \\
\text { SEPT } \\
\text { SEPI } \\
\text { SEPT } \\
\text { SEPT }\end{array}$ & $\begin{array}{l}13 \\
13 \\
13 \\
13 \\
13\end{array}$ & $\begin{array}{l}1439 \\
1440 \\
1442 \\
1445 \\
1450\end{array}$ & $\begin{array}{l}68 \\
64 \\
57 \\
46 \\
52\end{array}$ & $\begin{array}{l}37,900 \\
3+.300 \\
31,500 \\
25,100 \\
1, .300\end{array}$ & $\begin{array}{l}6,960 \\
5,930 \\
4,850 \\
3,120 \\
2,430\end{array}$ & $\begin{array}{l}27 \\
26 \\
25 \\
23 \\
20\end{array}$ & $\begin{array}{l}36 \\
35 \\
34 \\
31 \\
27\end{array}$ & $\begin{array}{l}+7 \\
46 \\
45 \\
11 \\
36\end{array}$ & $\begin{array}{l}62 \\
61 \\
60 \\
51 \\
17\end{array}$ & $\begin{array}{l}79 \\
77 \\
76 \\
70 \\
61\end{array}$ & $\begin{array}{l}86 \\
85 \\
83 \\
79 \\
73\end{array}$ & $\begin{array}{l}95 \\
94 \\
93 \\
92 \\
89\end{array}$ & $\begin{array}{l}99 \\
99 \\
99 \\
98 \\
97\end{array}$ & $\begin{array}{r}100 \\
100 \\
100 \\
100 \\
99\end{array}$ & $\begin{array}{l}100 \\
100 \\
100 \\
100 \\
100\end{array}$ \\
\hline $\begin{array}{l}\text { SEPT } \\
\text { SEPT } \\
\text { SEPT }\end{array}$ & $\begin{array}{l}13 \\
13 \\
13\end{array}$ & $\begin{array}{l}1455 \\
1500 \\
1505\end{array}$ & $\begin{array}{l}59 \\
65 \\
61\end{array}$ & $\begin{array}{r}16.600 \\
13.200 \\
8.980\end{array}$ & $\begin{array}{l}2,650 \\
2,320 \\
1,430\end{array}$ & $\begin{array}{l}11 \\
12 \\
y\end{array}$ & $\begin{array}{l}15 \\
14 \\
13\end{array}$ & $\begin{array}{l}21 \\
19 \\
19\end{array}$ & $\begin{array}{l}30 \\
28 \\
29\end{array}$ & $\begin{array}{l}42 \\
41 \\
46\end{array}$ & $\begin{array}{l}57 \\
55 \\
65\end{array}$ & $\begin{array}{l}8 \hat{3} \\
82 \\
87\end{array}$ & $\begin{array}{l}96 \\
96 \\
98\end{array}$ & $\begin{array}{r}99 \\
99 \\
100\end{array}$ & $\begin{array}{l}100 \\
100 \\
100\end{array}$ \\
\hline & & & & & Average & 27 & 36 & 47 & 02 & $=\bar{y}$ & 86 & 95 & 99 & 100 & 100 \\
\hline
\end{tabular}

50051180 Quebrada Salvatierra near San Lorenzo

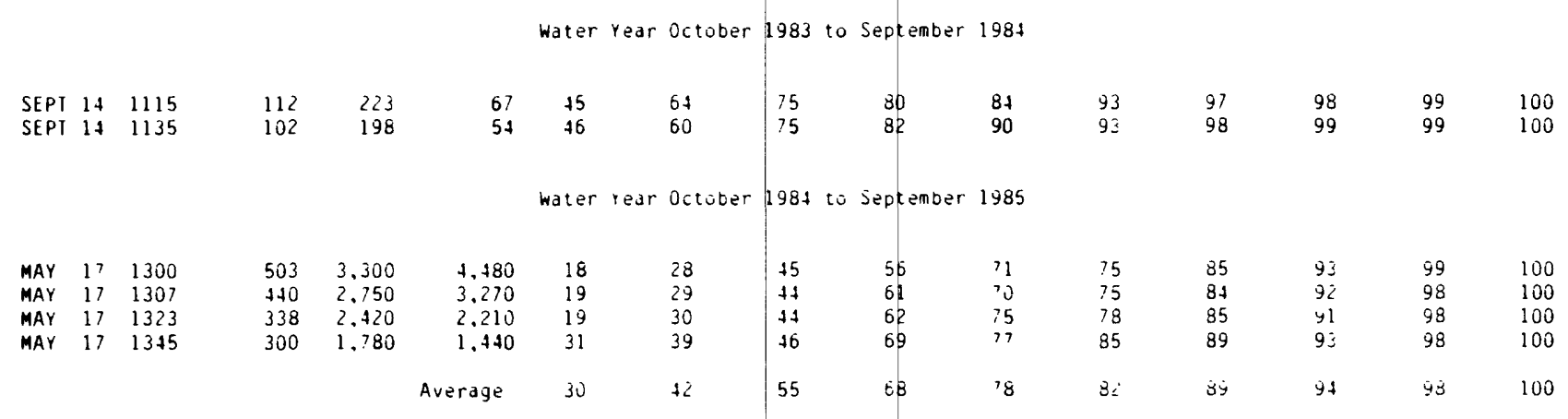




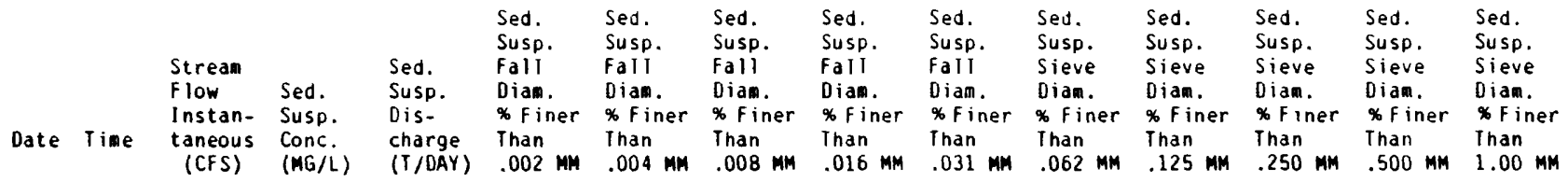

50051310 Rio Cayaguas at Cerro Gordo

Water Year October 1984 to September 1985

\begin{tabular}{|c|c|c|c|c|c|c|c|c|c|c|c|c|c|c|c|}
\hline $\begin{array}{l}\text { NOV } \\
\text { NOV } \\
\text { MAY } \\
\text { MAY } \\
\text { MAY }\end{array}$ & $\begin{array}{r}5 \\
5 \\
15 \\
15 \\
15\end{array}$ & $\begin{array}{l}1410 \\
1410 \\
1300 \\
1305 \\
1315\end{array}$ & $\begin{array}{l}1,210 \\
1,210 \\
1,240 \\
1,390 \\
1,670\end{array}$ & $\begin{array}{l}4.200 \\
4.100 \\
3.040 \\
3.790 \\
4.450\end{array}$ & $\begin{array}{l}13,800 \\
13,400 \\
10.200 \\
14.200 \\
20,100\end{array}$ & $\begin{array}{l}15 \\
12 \\
12 \\
13 \\
15\end{array}$ & $\begin{array}{l}24 \\
18 \\
18 \\
16 \\
20\end{array}$ & $\begin{array}{l}35 \\
28 \\
28 \\
25 \\
31\end{array}$ & $\begin{array}{l}46 \\
42 \\
53 \\
37 \\
49\end{array}$ & $\begin{array}{l}61 \\
56 \\
62 \\
54 \\
67\end{array}$ & $\begin{array}{l}70 \\
65 \\
70 \\
60 \\
75\end{array}$ & $\begin{array}{l}81 \\
79 \\
88 \\
83 \\
87\end{array}$ & $\begin{array}{l}90 \\
91 \\
95 \\
93 \\
94\end{array}$ & $\begin{array}{l}96 \\
98 \\
98 \\
97 \\
96\end{array}$ & $\begin{array}{l}99 \\
99 \\
99 \\
99 \\
98\end{array}$ \\
\hline $\begin{array}{l}\text { MAY } \\
\text { MAY } \\
\text { MAY } \\
\text { MAY } \\
\text { MAY }\end{array}$ & $\begin{array}{l}15 \\
15 \\
15 \\
18 \\
18\end{array}$ & $\begin{array}{l}1330 \\
1345 \\
1400 \\
1100 \\
1106\end{array}$ & $\begin{array}{l}1,910 \\
2,580 \\
3,240 \\
2,100 \\
2,040\end{array}$ & $\begin{array}{l}3,780 \\
3,530 \\
5,080 \\
4,530 \\
3,220\end{array}$ & $\begin{array}{l}19,500 \\
24,600 \\
44,400 \\
25,700 \\
17,700\end{array}$ & $\begin{array}{r}9 \\
12 \\
7 \\
6 \\
1\end{array}$ & $\begin{array}{r}12 \\
13 \\
11 \\
8 \\
12\end{array}$ & $\begin{array}{l}22 \\
24 \\
20 \\
17 \\
23\end{array}$ & $\begin{array}{l}36 \\
38 \\
33 \\
24 \\
35\end{array}$ & $\begin{array}{l}52 \\
56 \\
45 \\
33 \\
45\end{array}$ & $\begin{array}{l}71 \\
67 \\
55 \\
37 \\
52\end{array}$ & $\begin{array}{l}81 \\
82 \\
72 \\
55 \\
67\end{array}$ & $\begin{array}{l}88 \\
91 \\
83 \\
76 \\
83\end{array}$ & $\begin{array}{l}93 \\
96 \\
89 \\
91 \\
92\end{array}$ & $\begin{array}{l}96 \\
99 \\
94 \\
98 \\
98\end{array}$ \\
\hline $\begin{array}{l}\text { MAY } \\
\text { SEPT } \\
\text { SEPT } \\
\text { SEPT }\end{array}$ & $\begin{array}{l}18 \\
12 \\
12 \\
12\end{array}$ & $\begin{array}{l}1145 \\
1935 \\
2020 \\
2035\end{array}$ & $\begin{array}{l}1,510 \\
2,270 \\
1,920 \\
1,750\end{array}$ & $\begin{array}{l}3,030 \\
6,560 \\
6,910 \\
6,720\end{array}$ & $\begin{array}{l}12,400 \\
40,200 \\
35,800 \\
31,800\end{array}$ & $\begin{array}{r}11 \\
8 \\
11 \\
11\end{array}$ & $\begin{array}{l}15 \\
13 \\
18 \\
20\end{array}$ & $\begin{array}{l}27 \\
29 \\
28 \\
31\end{array}$ & $\begin{array}{l}38 \\
42 \\
48 \\
50\end{array}$ & $\begin{array}{l}50 \\
59 \\
63 \\
68\end{array}$ & $\begin{array}{l}58 \\
68 \\
69 \\
74\end{array}$ & $\begin{array}{l}75 \\
89 \\
92 \\
92\end{array}$ & $\begin{array}{l}86 \\
99 \\
98 \\
99\end{array}$ & $\begin{array}{r}96 \\
100 \\
100 \\
100\end{array}$ & $\begin{array}{r}99 \\
100 \\
100 \\
100\end{array}$ \\
\hline
\end{tabular}

Water Year October 1985 to September 1986

$\begin{array}{lll}\text { OCT } & 6 & 1115 \\ \text { OCT } & 6 & 1200 \\ \text { OCT } & 6 & 1230 \\ \text { OCT } & 6 & 1300\end{array}$

$\begin{array}{ll}3,140 & 6,270 \\ 2,910 & 7,090 \\ 2,710 & 6.530 \\ 2,640 & 5.680\end{array}$

53,100

55,700

47,800

45,500

7
8
10
12

9
16
16
18

$\begin{array}{ll}16 & 31 \\ 23 & 38 \\ 29 & 39 \\ 31 & 42\end{array}$

$\begin{array}{ll}31 & 45 \\ 38 & 54 \\ 39 & 50 \\ 42 & 57\end{array}$
$15 \quad 26$

$40 \quad 54$
50
62
57
62

62

Water Year October 1984 to September 1985

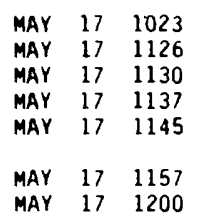

$17 \quad 1023$

MAY $17 \quad 1130$

MAY $17 \quad 1137$

MAY $17 \quad 1157$

MAY $17 \quad 1200$

$\begin{array}{rrr}3,370 & 4,700 & 42,800 \\ 6,110 & 26,000 & 429,000 \\ 6,110 & 28,600 & 472,000 \\ 6,120 & 26,900 & 445,000 \\ 6,130 & 20,300 & 336,000 \\ & & \\ 6,130 & 13,800 & 228,000 \\ 6,130 & 11,800 & 195,000\end{array}$

$\begin{array}{rrr}2 & 13 & 21 \\ 6 & 9 & 1 \\ 8 & 12 & 1 \\ 6 & 10 & 15 \\ 8 & 12 & 23 \\ 9 & 16 & 25 \\ 14 & 21 & 32\end{array}$

$\begin{array}{lll}21 & 32 & 48 \\ 12 & 16 & 21 \\ 16 & 16 & 28 \\ 15 & 20 & 25 \\ 23 & 33 & 45 \\ & & \\ 25 & 35 & 48 \\ 32 & 46 & 58\end{array}$

$\begin{array}{ll}48 & 59 \\ 21 & 43 \\ 28 & 55 \\ 25 & 38 \\ 45 & 56 \\ 48 & 5 \\ 58 & 6\end{array}$

Water Year October 1985 to September 1986

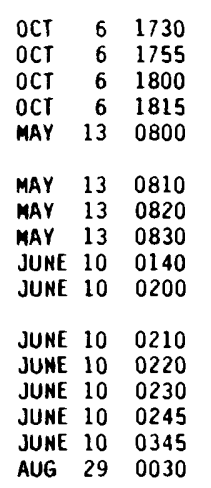

$\begin{array}{rr}34,700 & 8 \\ 24,500 & 11 \\ 26,800 & 11 \\ 18,300 & 12 \\ 94,500 & 4 \\ 90,300 & 4 \\ 52,300 & 6 \\ 58,300 & 4 \\ 6,720 & 10 \\ 36,100 & 9 \\ 26,400 & 12 \\ 24,400 & 11 \\ 18,100 & 12 \\ 10,600 & 13 \\ 2,030 & 16 \\ 2,970 & 9\end{array}$

Average

$\begin{array}{rr}2,640 & 4,870 \\ 1,990 & 4,560 \\ 1,860 & 5,340 \\ 1,830 & 5,700 \\ 1,700 & 20,600 \\ & \\ 1,990 & 16,800 \\ 1,970 & 9,840 \\ 1,660 & 13,000 \\ 496 & 5,020 \\ 916 & 14,600 \\ & \\ 985 & 9,940 \\ 920 & 9,840 \\ 728 & 9,230 \\ 501 & 7,870 \\ 234 & 3,220 \\ 257 & 4,280\end{array}$

$\begin{array}{rr}8 & 13 \\ 11 & 17 \\ 11 & 17 \\ 12 & 21 \\ 4 & 6 \\ 4 & \\ 6 & 10 \\ 4 & 7 \\ 10 & 19 \\ 9 & 17 \\ & \\ 12 & 22 \\ 11 & 18 \\ 12 & 22 \\ 13 & 23 \\ 16 & 24 \\ 9 & 13\end{array}$

$125 \mathrm{mM} 250 \mathrm{mM} .500 \mathrm{MM} 1.00 \mathrm{mM}$ 


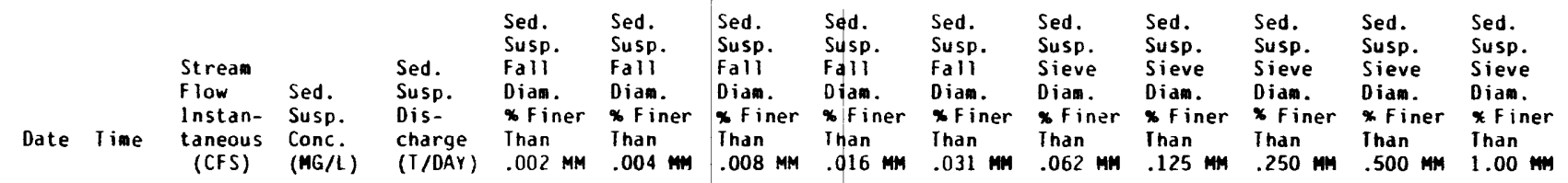

50055000 Rio Grande de Loiza at Caguas

Water Year October 1984 to September 1985

\begin{tabular}{|c|c|c|c|c|c|c|c|c|c|c|c|c|c|c|c|}
\hline $\begin{array}{l}\text { MOV } \\
\text { MOV } \\
\text { MAY } \\
\text { MAY } \\
\text { MAY }\end{array}$ & $\begin{array}{r}5 \\
5 \\
15 \\
15 \\
15\end{array}$ & $\begin{array}{l}1745 \\
1830 \\
1545 \\
1555 \\
1605\end{array}$ & $\begin{array}{r}7,620 \\
7,520 \\
11,600 \\
11,300 \\
11,000\end{array}$ & $\begin{array}{l}3,500 \\
3,060 \\
4,120 \\
4,400 \\
4,200\end{array}$ & $\begin{array}{r}72,000 \\
62,000 \\
129,000 \\
134.000 \\
125,000\end{array}$ & $\begin{array}{r}20 \\
15 \\
5 \\
6 \\
9\end{array}$ & $\begin{array}{r}27 \\
23 \\
9 \\
11 \\
12\end{array}$ & $\begin{array}{l}34 \\
32 \\
19 \\
19 \\
23\end{array}$ & $\begin{array}{l}10 \\
45 \\
30 \\
28 \\
33\end{array}$ & $\begin{array}{l}42 \\
59 \\
46 \\
43 \\
48\end{array}$ & $\begin{array}{l}53 \\
67 \\
59 \\
56 \\
59\end{array}$ & $\begin{array}{l}66 \\
83 \\
77 \\
72 \\
79\end{array}$ & $\begin{array}{l}83 \\
94 \\
92 \\
89 \\
90\end{array}$ & $\begin{array}{l}92 \\
97 \\
98 \\
96 \\
96\end{array}$ & $\begin{array}{r}98 \\
100 \\
99 \\
99 \\
99\end{array}$ \\
\hline $\begin{array}{l}\text { MAY } \\
\text { MAY } \\
\text { MAY } \\
\text { MAY } \\
\text { MAY }\end{array}$ & $\begin{array}{l}15 \\
15 \\
15 \\
15 \\
15\end{array}$ & $\begin{array}{l}1615 \\
1625 \\
1635 \\
1645 \\
1655\end{array}$ & $\begin{array}{r}10,500 \\
9,920 \\
9,310 \\
8,670 \\
7,400\end{array}$ & $\begin{array}{l}3,280 \\
3,400 \\
3,200 \\
2,220 \\
2,820\end{array}$ & $\begin{array}{l}93,600 \\
91,100 \\
80,400 \\
51,500 \\
56,300\end{array}$ & $\begin{array}{l}9 \\
10 \\
11 \\
16 \\
14\end{array}$ & $\begin{array}{l}15 \\
17 \\
16 \\
23 \\
22\end{array}$ & $\begin{array}{l}27 \\
27 \\
27 \\
35 \\
34\end{array}$ & $\begin{array}{l}40 \\
12 \\
17 \\
51 \\
50\end{array}$ & $\begin{array}{l}59 \\
58 \\
58 \\
68 \\
67\end{array}$ & $\begin{array}{l}68 \\
71 \\
69 \\
75 \\
74\end{array}$ & $\begin{array}{l}83 \\
85 \\
86 \\
88 \\
88\end{array}$ & $\begin{array}{l}95 \\
95 \\
95 \\
97 \\
95\end{array}$ & $\begin{array}{l}98 \\
98 \\
98 \\
99 \\
98\end{array}$ & $\begin{array}{r}99 \\
99 \\
99 \\
100 \\
99\end{array}$ \\
\hline $\begin{array}{l}\text { MAY } \\
\text { MAY } \\
\text { MAY } \\
\text { MAY } \\
\text { MAY }\end{array}$ & $\begin{array}{l}17 \\
17 \\
17 \\
17 \\
17\end{array}$ & $\begin{array}{l}1200 \\
1205 \\
1215 \\
1220 \\
1225\end{array}$ & $\begin{array}{l}16,500 \\
18,200 \\
21,500 \\
22,600 \\
23,700\end{array}$ & $\begin{array}{r}12,200 \\
11,200 \\
9,940 \\
10,700 \\
9,820\end{array}$ & $\begin{array}{l}544,000 \\
550,000 \\
577,000 \\
653,000 \\
626,000\end{array}$ & $\begin{array}{r}9 \\
13 \\
16 \\
9 \\
12\end{array}$ & $\begin{array}{l}15 \\
21 \\
24 \\
14 \\
19\end{array}$ & $\begin{array}{l}20 \\
30 \\
33 \\
21 \\
26\end{array}$ & $\begin{array}{l}29 \\
41 \\
46 \\
30 \\
36\end{array}$ & $\begin{array}{l}38 \\
55 \\
60 \\
39 \\
48\end{array}$ & $\begin{array}{l}69 \\
73 \\
79 \\
74 \\
76\end{array}$ & $\begin{array}{l}83 \\
87 \\
90 \\
85 \\
88\end{array}$ & $\begin{array}{l}96 \\
96 \\
97 \\
94 \\
96\end{array}$ & $\begin{array}{l}99 \\
99 \\
99 \\
98 \\
99\end{array}$ & $\begin{array}{r}100 \\
100 \\
100 \\
99 \\
100\end{array}$ \\
\hline $\begin{array}{l}\text { MAY } \\
\text { MAY } \\
\text { MAY } \\
\text { MAY } \\
\text { MAY }\end{array}$ & $\begin{array}{l}17 \\
17 \\
17 \\
17 \\
17\end{array}$ & $\begin{array}{l}1230 \\
1235 \\
1240 \\
1245 \\
1250\end{array}$ & $\begin{array}{l}24,800 \\
23,500 \\
22,300 \\
24,600 \\
23,700\end{array}$ & $\begin{array}{r}10,200 \\
9,970 \\
9,930 \\
12,300 \\
8,610\end{array}$ & $\begin{array}{l}683,000 \\
634,000 \\
597,000 \\
817,000 \\
551,000\end{array}$ & $\begin{array}{r}10 \\
9 \\
8 \\
8 \\
10\end{array}$ & $\begin{array}{l}17 \\
17 \\
15 \\
17 \\
17\end{array}$ & $\begin{array}{l}25 \\
28 \\
24 \\
26 \\
30\end{array}$ & $\begin{array}{l}34 \\
39 \\
37 \\
38 \\
43\end{array}$ & $\begin{array}{l}44 \\
56 \\
48 \\
50 \\
59\end{array}$ & $\begin{array}{l}7 j \\
74 \\
71 \\
74 \\
75\end{array}$ & $\begin{array}{l}85 \\
88 \\
87 \\
89 \\
89\end{array}$ & $\begin{array}{l}93 \\
95 \\
95 \\
97 \\
96\end{array}$ & $\begin{array}{l}99 \\
99 \\
98 \\
99 \\
99\end{array}$ & $\begin{array}{r}100 \\
100 \\
99 \\
100 \\
100\end{array}$ \\
\hline $\begin{array}{l}\text { MAY } \\
\text { MAY } \\
\text { MAY } \\
\text { MAY } \\
\text { MAY }\end{array}$ & $\begin{array}{l}17 \\
17 \\
17 \\
17 \\
17\end{array}$ & $\begin{array}{l}1300 \\
1316 \\
1320 \\
1330 \\
1345\end{array}$ & $\begin{array}{l}21,800 \\
19,400 \\
18,400 \\
16,100 \\
13,700\end{array}$ & $\begin{array}{l}8,400 \\
7,400 \\
6,900 \\
6,600 \\
6,300\end{array}$ & $\begin{array}{l}494,000 \\
388,000 \\
343,000 \\
287,000 \\
233,000\end{array}$ & $\begin{array}{r}7 \\
10 \\
12 \\
14 \\
13\end{array}$ & $\begin{array}{l}12 \\
19 \\
19 \\
22 \\
21\end{array}$ & $\begin{array}{l}21 \\
32 \\
22 \\
35 \\
36\end{array}$ & $\begin{array}{l}30 \\
44 \\
44 \\
46 \\
49\end{array}$ & $\begin{array}{l}43 \\
60 \\
59 \\
64 \\
64\end{array}$ & $\begin{array}{l}76 \\
82 \\
87 \\
82 \\
81\end{array}$ & $\begin{array}{l}88 \\
92 \\
94 \\
92 \\
92\end{array}$ & $\begin{array}{l}94 \\
97 \\
96 \\
98 \\
98\end{array}$ & $\begin{array}{r}98 \\
100 \\
99 \\
99 \\
99\end{array}$ & $\begin{array}{l}98 \\
100 \\
100 \\
100 \\
100\end{array}$ \\
\hline $\begin{array}{l}\text { MAY } \\
\text { MAY } \\
\text { MAY } \\
\text { MAY } \\
\text { MAY }\end{array}$ & $\begin{array}{l}17 \\
17 \\
17 \\
17 \\
17\end{array}$ & $\begin{array}{l}1400 \\
1415 \\
1430 \\
1445 \\
1500\end{array}$ & $\begin{array}{r}11,600 \\
9,870 \\
8,450 \\
7,500 \\
6,760\end{array}$ & $\begin{array}{l}5,660 \\
5,100 \\
4,700 \\
4,680 \\
3,120\end{array}$ & $\begin{array}{r}177,000 \\
136,000 \\
107,000 \\
94,800 \\
57,000\end{array}$ & $\begin{array}{l}13 \\
13 \\
12 \\
12 \\
13\end{array}$ & $\begin{array}{l}21 \\
21 \\
23 \\
21 \\
20\end{array}$ & $\begin{array}{l}31 \\
35 \\
31 \\
32 \\
30\end{array}$ & $\begin{array}{l}50 \\
52 \\
45 \\
46 \\
53\end{array}$ & $\begin{array}{l}63 \\
70 \\
69 \\
66 \\
67\end{array}$ & $\begin{array}{l}79 \\
80 \\
78 \\
80 \\
84\end{array}$ & $\begin{array}{l}93 \\
94 \\
91 \\
93 \\
94\end{array}$ & $\begin{array}{l}98 \\
98 \\
97 \\
98 \\
98\end{array}$ & $\begin{array}{r}100 \\
100 \\
99 \\
99 \\
99\end{array}$ & $\begin{array}{l}100 \\
100 \\
100 \\
100 \\
100\end{array}$ \\
\hline $\begin{array}{l}\text { MAY } \\
\text { MAY } \\
\text { MAY } \\
\text { MAY } \\
\text { MAY }\end{array}$ & $\begin{array}{l}17 \\
17 \\
17 \\
18 \\
18\end{array}$ & $\begin{array}{l}1502 \\
1515 \\
1545 \\
1000 \\
1030\end{array}$ & $\begin{array}{r}6,700 \\
6,270 \\
5,260 \\
18,600 \\
16,700\end{array}$ & $\begin{array}{l}3,440 \\
3,660 \\
3,090 \\
4,500 \\
3,700\end{array}$ & $\begin{array}{r}62,200 \\
62,000 \\
43,900 \\
226,000 \\
167,000\end{array}$ & $\begin{array}{l}12 \\
22 \\
16 \\
15 \\
16\end{array}$ & $\begin{array}{l}18 \\
31 \\
25 \\
23 \\
23\end{array}$ & $\begin{array}{l}33 \\
41 \\
40 \\
35 \\
30\end{array}$ & $\begin{array}{l}54 \\
53 \\
56 \\
50 \\
52\end{array}$ & $\begin{array}{l}72 \\
76 \\
77 \\
65 \\
64\end{array}$ & $\begin{array}{l}84 \\
88 \\
86 \\
75 \\
71\end{array}$ & $\begin{array}{l}93 \\
96 \\
96 \\
89 \\
83\end{array}$ & $\begin{array}{l}98 \\
99 \\
99 \\
91 \\
95\end{array}$ & $\begin{array}{r}99 \\
100 \\
100 \\
99 \\
98\end{array}$ & $\begin{array}{r}100 \\
100 \\
100 \\
99 \\
99\end{array}$ \\
\hline $\begin{array}{l}\text { MAY } \\
\text { MAY }\end{array}$ & $\begin{array}{l}18 \\
18\end{array}$ & $\begin{array}{l}1200 \\
1232\end{array}$ & $\begin{array}{r}11,300 \\
9,320\end{array}$ & $\begin{array}{l}4,320 \\
4,200\end{array}$ & $\begin{array}{l}131,000 \\
106,000\end{array}$ & $\begin{array}{l}10 \\
14\end{array}$ & $\begin{array}{l}11 \\
27\end{array}$ & $\begin{array}{l}24 \\
28\end{array}$ & $\begin{array}{l}33 \\
43\end{array}$ & $\begin{array}{l}45 \\
49\end{array}$ & $\begin{array}{l}52 \\
52\end{array}$ & $\begin{array}{l}72 \\
78\end{array}$ & $\begin{array}{l}88 \\
93\end{array}$ & $\begin{array}{l}96 \\
98\end{array}$ & $\begin{array}{r}99 \\
100\end{array}$ \\
\hline
\end{tabular}

Water Year October 1985 to September 1986

\begin{tabular}{|c|c|c|c|c|c|c|c|c|c|c|c|c|c|c|c|}
\hline $\begin{array}{l}O C T \\
O C T \\
0 C T \\
O C T \\
O C T\end{array}$ & $\begin{array}{l}6 \\
6 \\
6 \\
6 \\
6\end{array}$ & $\begin{array}{l}1245 \\
1430 \\
1645 \\
1700 \\
1715\end{array}$ & $\begin{array}{l}13.300 \\
12.800 \\
15.700 \\
17.600 \\
16.900\end{array}$ & $\begin{array}{l}4,520 \\
3,590 \\
6,180 \\
9,220 \\
8,680\end{array}$ & $\begin{array}{l}162,000 \\
124,000 \\
262,000 \\
438,000 \\
396,000\end{array}$ & $\begin{array}{r}10 \\
11 \\
10 \\
9 \\
8\end{array}$ & $\begin{array}{l}16 \\
17 \\
15 \\
15 \\
16\end{array}$ & $\begin{array}{l}25 \\
24 \\
19 \\
23 \\
25\end{array}$ & $\begin{array}{l}37 \\
34 \\
33 \\
32 \\
35\end{array}$ & $\begin{array}{l}50 \\
46 \\
46 \\
45 \\
47\end{array}$ & $\begin{array}{l}58 \\
52 \\
55 \\
62 \\
60\end{array}$ & $\begin{array}{l}79 \\
71 \\
74 \\
76 \\
75\end{array}$ & $\begin{array}{l}94 \\
91 \\
96 \\
96 \\
92\end{array}$ & $\begin{array}{r}99 \\
98 \\
99 \\
100 \\
99\end{array}$ & $\begin{array}{l}100 \\
100 \\
100 \\
100 \\
100\end{array}$ \\
\hline $\begin{array}{l}\text { OCT } \\
\text { OCT } \\
\text { OCI } \\
\text { OCT } \\
\text { APR }\end{array}$ & $\begin{array}{r}6 \\
6 \\
6 \\
6 \\
29\end{array}$ & $\begin{array}{l}1730 \\
1745 \\
1800 \\
1815 \\
1645\end{array}$ & $\begin{array}{r}16,900 \\
16,000 \\
15,600 \\
15,500 \\
7,270\end{array}$ & $\begin{array}{l}6,840 \\
+, 890 \\
4,220 \\
3,580 \\
6,290\end{array}$ & $\begin{array}{l}312,000 \\
211,000 \\
178,000 \\
150,000 \\
123,000\end{array}$ & $\begin{array}{l}11 \\
14 \\
12 \\
14 \\
13\end{array}$ & $\begin{array}{l}19 \\
22 \\
20 \\
24 \\
20\end{array}$ & $\begin{array}{l}29 \\
32 \\
27 \\
35 \\
29\end{array}$ & $\begin{array}{l}40 \\
46 \\
39 \\
19 \\
46\end{array}$ & $\begin{array}{l}52 \\
60 \\
51 \\
61 \\
69\end{array}$ & $\begin{array}{l}\text { b3 } \\
69 \\
59 \\
72 \\
86\end{array}$ & $\begin{array}{l}78 \\
86 \\
76 \\
87 \\
94\end{array}$ & $\begin{array}{l}94 \\
97 \\
93 \\
98 \\
99\end{array}$ & $\begin{array}{r}9 y \\
100 \\
99 \\
100 \\
100\end{array}$ & $\begin{array}{l}100 \\
100 \\
100 \\
100 \\
100\end{array}$ \\
\hline $\begin{array}{l}A P R \\
\text { APR } \\
\text { MAi } \\
\text { MAY } \\
\text { MAY }\end{array}$ & $\begin{array}{r}29 \\
29 \\
8 \\
8 \\
8\end{array}$ & $\begin{array}{l}1700 \\
1715 \\
1715 \\
1730 \\
1745\end{array}$ & $\begin{array}{r}8,930 \\
9,370 \\
13,900 \\
14,100 \\
13,200\end{array}$ & $\begin{array}{l}5,960 \\
4,860 \\
6,990 \\
5,880 \\
5,500\end{array}$ & $\begin{array}{l}144,000 \\
123,000 \\
262,000 \\
224,000 \\
196,000\end{array}$ & $\begin{array}{l}12 \\
13 \\
13 \\
14 \\
13\end{array}$ & $\begin{array}{l}15 \\
17 \\
21 \\
15 \\
22\end{array}$ & $\begin{array}{l}25 \\
36 \\
32 \\
34 \\
33\end{array}$ & $\begin{array}{l}39 \\
41 \\
46 \\
48 \\
17\end{array}$ & $\begin{array}{l}60 \\
63 \\
62 \\
63 \\
59\end{array}$ & $\begin{array}{l}78 \\
80 \\
80 \\
77 \\
70\end{array}$ & $\begin{array}{l}94 \\
93 \\
95 \\
92 \\
90\end{array}$ & $\begin{array}{l}98 \\
99 \\
99 \\
99 \\
99\end{array}$ & $\begin{array}{r}100 \\
100 \\
100 \\
99 \\
100\end{array}$ & $\begin{array}{l}100 \\
100 \\
100 \\
100 \\
100\end{array}$ \\
\hline $\begin{array}{l}\text { MAY } \\
\text { MAY } \\
\text { MAY } \\
\text { MAY }\end{array}$ & $\begin{array}{r}8 \\
13 \\
13 \\
13\end{array}$ & $\begin{array}{l}1830 \\
0845 \\
0915 \\
0945\end{array}$ & $\begin{array}{r}9,620 \\
10,600 \\
15,200 \\
16,300\end{array}$ & $\begin{array}{l}3,540 \\
2,600 \\
5,190 \\
5,550\end{array}$ & $\begin{array}{r}92,000 \\
74,400 \\
213,000 \\
244,000\end{array}$ & $\begin{array}{r}16 \\
16 \\
11 \\
7\end{array}$ & $\begin{array}{l}24 \\
27 \\
19 \\
13\end{array}$ & $\begin{array}{l}38 \\
40 \\
27 \\
19\end{array}$ & $\begin{array}{l}53 \\
58 \\
40 \\
27\end{array}$ & $\begin{array}{l}72 \\
78 \\
51 \\
36\end{array}$ & $\begin{array}{l}80 \\
85 \\
62 \\
43\end{array}$ & $\begin{array}{l}94 \\
98 \\
82 \\
63\end{array}$ & $\begin{array}{l}99 \\
99 \\
88 \\
94\end{array}$ & $\begin{array}{r}100 \\
100 \\
99 \\
100\end{array}$ & $\begin{array}{l}100 \\
100 \\
100 \\
100\end{array}$ \\
\hline & & & & & Average & 12 & $1 \bar{y}$ & 29 & 43 & 58 & 73 & 87 & 96 & 99 & 100 \\
\hline
\end{tabular}


Table 15. Particle-size distribution data fro suspended-sedinent sanples collected at ten surface-water stations.in Rio Grande de Loiza basin--Cont inued

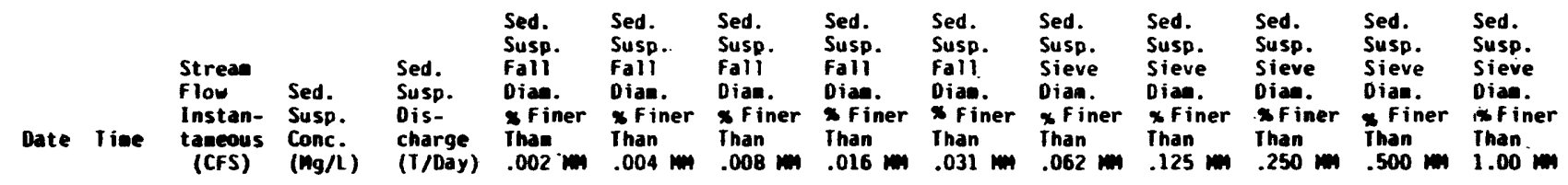

50055650 Quebrada Cainito near Juncos

Water Year October 1984 to Septeaber 1985

\begin{tabular}{|c|c|c|c|c|c|c|c|c|c|c|c|c|c|c|c|}
\hline nov & 5 & 1555 & 116 & 2,470 & 774 & 25 & 26 & 38 & 53 & 65 & $8 z$ & 91 & 96 & 98 & 99 \\
\hline & & & & & & ater & Oct & 1985 & epte & 1986 & & & & & \\
\hline \multirow[t]{2}{*}{ DCI } & 24 & 1115 & 29 & 1,980 & 155 & 32 & 40 & 52 & 81 & 93 & 94 & 97 & 99 & 100 & 100 \\
\hline & & & & & age & 28 & 33 & 45 & 67 & 79 & 88 & 94 & 98 & 99 & 100 \\
\hline
\end{tabular}

50056400 Rio Valenciano near Juncos

Water Year October 1984 to September 1985

\begin{tabular}{|c|c|c|c|c|c|c|c|c|c|c|c|c|c|c|c|}
\hline $\begin{array}{l}\text { SEPT } \\
\text { SEPT }\end{array}$ & $\begin{array}{l}12 \\
12\end{array}$ & $\begin{array}{l}1835 \\
1920\end{array}$ & $\begin{array}{r}577 \\
1,940\end{array}$ & $\begin{array}{l}1,550 \\
2,030\end{array}$ & $\begin{array}{r}2,400 \\
10,600\end{array}$ & $\begin{array}{l}11 \\
11\end{array}$ & $\begin{array}{l}17 \\
16\end{array}$ & $\begin{array}{l}29 \\
26\end{array}$ & $\begin{array}{l}49 \\
41\end{array}$ & $\begin{array}{l}68 \\
58\end{array}$ & $\begin{array}{l}74 \\
69\end{array}$ & $\begin{array}{l}89 \\
84\end{array}$ & $\begin{array}{l}98 \\
96\end{array}$ & $\begin{array}{l}100 \\
100\end{array}$ & $\begin{array}{l}100 \\
100\end{array}$ \\
\hline \multicolumn{16}{|c|}{ Water Year October 1985 to Septeaber 1986} \\
\hline $\begin{array}{l}\text { OCT } \\
\text { OCT } \\
\text { OCT } \\
\text { OCT } \\
\text { OCT }\end{array}$ & $\begin{array}{l}6 \\
6 \\
6 \\
6 \\
6\end{array}$ & $\begin{array}{l}0620 \\
0800 \\
0815 \\
0845 \\
1620\end{array}$ & $\begin{array}{r}616 \\
1,670 \\
2,100 \\
3,220 \\
5,600\end{array}$ & $\begin{array}{l}2,340 \\
2,320 \\
2,500 \\
2,480 \\
4,200\end{array}$ & $\begin{array}{r}4,800 \\
10,500 \\
14,100 \\
21,600 \\
63,500\end{array}$ & $\begin{array}{r}10 \\
10 \\
10 \\
14 \\
8\end{array}$ & $\begin{array}{r}11 \\
14 \\
14 \\
17 \\
8\end{array}$ & $\begin{array}{l}19 \\
21 \\
20 \\
23 \\
15\end{array}$ & $\begin{array}{l}28 \\
30 \\
28 \\
32 \\
26\end{array}$ & $\begin{array}{l}35 \\
38 \\
38 \\
44 \\
36\end{array}$ & $\begin{array}{l}41 \\
46 \\
44 \\
50 \\
38\end{array}$ & $\begin{array}{l}58 \\
63 \\
62 \\
68 \\
66\end{array}$ & $\begin{array}{l}84 \\
88 \\
85 \\
89 \\
80\end{array}$ & $\begin{array}{l}99 \\
99 \\
98 \\
98 \\
98\end{array}$ & $\begin{array}{l}100 \\
100 \\
100 \\
100 \\
100\end{array}$ \\
\hline $\begin{array}{l}\text { OCT } \\
\text { OCI } \\
\text { OCT } \\
\text { APR } \\
\text { APR }\end{array}$ & $\begin{array}{r}6 \\
6 \\
6 \\
29 \\
29\end{array}$ & $\begin{array}{l}1850 \\
1920 \\
1950 \\
1540 \\
1545\end{array}$ & $\begin{array}{l}4,900 \\
4,000 \\
3,200 \\
1,410 \\
2,070\end{array}$ & $\begin{array}{l}5,740 \\
3,950 \\
3,460 \\
3,200 \\
3,020\end{array}$ & $\begin{array}{l}76,000 \\
42,700 \\
29,900 \\
12,400 \\
16,900\end{array}$ & $\begin{array}{r}5 \\
9 \\
7 \\
14 \\
14\end{array}$ & $\begin{array}{l}8 \\
14 \\
18 \\
25 \\
20\end{array}$ & $\begin{array}{l}13 \\
22 \\
20 \\
32 \\
32\end{array}$ & $\begin{array}{l}21 \\
31 \\
32 \\
35 \\
47\end{array}$ & $\begin{array}{l}32 \\
47 \\
42 \\
70 \\
65\end{array}$ & $\begin{array}{l}41 \\
56 \\
48 \\
83 \\
78\end{array}$ & $\begin{array}{l}64 \\
77 \\
79 \\
96 \\
94\end{array}$ & $\begin{array}{l}86 \\
92 \\
93 \\
99 \\
99\end{array}$ & $\begin{array}{r}98 \\
98 \\
98 \\
100 \\
100\end{array}$ & $\begin{array}{l}100 \\
100 \\
100 \\
100 \\
100\end{array}$ \\
\hline \multirow[t]{2}{*}{$\begin{array}{l}\text { APR } \\
\text { APR } \\
\text { MAY } \\
\text { MAY }\end{array}$} & $\begin{array}{l}29 \\
29 \\
13 \\
13\end{array}$ & $\begin{array}{l}1635 \\
1720 \\
0300 \\
0830\end{array}$ & $\begin{array}{r}2,680 \\
1,780 \\
587 \\
4,100\end{array}$ & $\begin{array}{l}3,250 \\
1,470 \\
1,810 \\
8,970\end{array}$ & $\begin{array}{r}23,500 \\
7,060 \\
2,870 \\
99,300\end{array}$ & $\begin{array}{r}13 \\
26 \\
24 \\
4\end{array}$ & $\begin{array}{r}18 \\
33 \\
27 \\
6\end{array}$ & $\begin{array}{r}23 \\
47 \\
41 \\
9\end{array}$ & $\begin{array}{l}40 \\
67 \\
62 \\
14\end{array}$ & $\begin{array}{l}56 \\
81 \\
82 \\
20\end{array}$ & $\begin{array}{l}65 \\
90 \\
84 \\
27\end{array}$ & $\begin{array}{r}79 \\
-- \\
98 \\
45\end{array}$ & $\begin{array}{r}95 \\
--- \\
99 \\
76\end{array}$ & $\begin{array}{r}99 \\
-100 \\
97\end{array}$ & $\begin{array}{r}100 \\
100 \\
99\end{array}$ \\
\hline & & & & & Average & 15 & 20 & 29 & 41 & 55 & 62 & 78 & 92 & 99 & 100 \\
\hline
\end{tabular}

50056900 Quebrada Maney near Gurabo

Water Year October 1985 to September 1986

$\begin{array}{rrrrrrrrrrrrrrrrrrrrrr}\text { OCT } & 6 & 1535 & 604 & 1.360 & 2.220 & 23 & 26 & 43 & 60 & 64 & 77 & 90 & 94 & 97 & 99 \\ & & & & \text { Average } & 23 & 26 & 43 & 60 & 64 & 77 & 90 & 94 & 97 & 99\end{array}$

50057000 Rio Gurabo at Gurabo

Water Year October 1984 to September 1985

$\begin{array}{llllll}\text { MAY } & 15 & 1300 & 6,040 & 2,600 & 42,400 \\ \text { MAY } & 15 & 1315 & 6,500 & 2,020 & 35,500 \\ \text { MAY } & 15 & 1400 & 8,260 & 1,960 & 43,700\end{array}$

MAY $15 \quad 1400 \quad 8,260 \quad 1,960 \quad 43,700$

$\begin{array}{lllll}16 & 23 & 30 & 44 & 58 \\ 21 & 31 & 42 & 47 & 68\end{array}$

$\begin{array}{llllll}58 & 78 & 90 & 93 & 98 & 99 \\ 68 & 77 & 84 & 90 & 98 & 99 \\ 66 & 71 & 77 & 81 & 90 & 96 \\ 79 & 85 & 92 & 94 & 97 & 98\end{array}$

Water Year October 1985 to September 1986

\begin{tabular}{|c|c|c|c|c|c|c|c|c|c|c|c|c|c|c|c|}
\hline $\begin{array}{l}\text { OCT } \\
\text { OCT } \\
\text { OCT } \\
\text { OCT } \\
\text { OCT }\end{array}$ & $\begin{array}{l}6 \\
6 \\
6 \\
6 \\
6\end{array}$ & $\begin{array}{l}1330 \\
1400 \\
1415 \\
1430 \\
1530\end{array}$ & $\begin{array}{l}11,800 \\
11,800 \\
11,600 \\
11,600 \\
12,600\end{array}$ & $\begin{array}{l}1,570 \\
1,550 \\
1,560 \\
1,390 \\
1,360\end{array}$ & $\begin{array}{l}50,000 \\
49,400 \\
48,900 \\
43,500 \\
46,300\end{array}$ & $\begin{array}{l}26 \\
29 \\
27 \\
28 \\
27\end{array}$ & $\begin{array}{l}36 \\
38 \\
40 \\
36 \\
37\end{array}$ & $\begin{array}{l}52 \\
50 \\
53 \\
51 \\
53\end{array}$ & $\begin{array}{l}71 \\
66 \\
71 \\
74 \\
71\end{array}$ & $\begin{array}{l}79 \\
88 \\
85 \\
84 \\
84\end{array}$ & $\begin{array}{l}82 \\
92 \\
86 \\
86 \\
88\end{array}$ & $\begin{array}{l}93 \\
97 \\
92 \\
95 \\
97\end{array}$ & $\begin{array}{l}96 \\
98 \\
93 \\
97 \\
99\end{array}$ & $\begin{array}{l}98 \\
99 \\
99 \\
98 \\
99\end{array}$ & $\begin{array}{l}100 \\
100 \\
100 \\
100 \\
100\end{array}$ \\
\hline \multirow[t]{2}{*}{$\begin{array}{l}\text { OCT } \\
\text { OCT }\end{array}$} & $\begin{array}{l}6 \\
6\end{array}$ & $\begin{array}{l}1630 \\
1700\end{array}$ & $\begin{array}{l}14,500 \\
15,600\end{array}$ & $\begin{array}{l}1,460 \\
1,420\end{array}$ & $\begin{array}{l}57.200 \\
59.800\end{array}$ & $\begin{array}{l}24 \\
32\end{array}$ & $\begin{array}{l}31 \\
40\end{array}$ & $\begin{array}{l}48 \\
56\end{array}$ & $\begin{array}{l}63 \\
73\end{array}$ & $\begin{array}{l}81 \\
86\end{array}$ & $\begin{array}{l}88 \\
92\end{array}$ & $\begin{array}{l}98 \\
98\end{array}$ & $\begin{array}{r}99 \\
100\end{array}$ & $\begin{array}{l}100 \\
100\end{array}$ & $\begin{array}{l}100 \\
100\end{array}$ \\
\hline & & & & & verage & 26 & 34 & 47 & 63 & 78 & 84 & ?? & 95 & 98 & 99 \\
\hline
\end{tabular}


Table 16.--Average water discharge for stations in the Río Grande de Loiza basin.

\begin{tabular}{ccc|cc}
\hline Station Number & Discharge, in $\mathrm{ft}^{3} / \mathrm{s}$ & Station Number & Discharge, in $\mathrm{ft}^{3} / \mathrm{s}$ \\
\hline 50050900 & 31.1 & 50055000 & 224 \\
051180 & 9.18 & 055650 & 1.32 \\
051180 & 7.92 & 056400 & 51.3 \\
051310 & 49.5 & 056900 & 4.43 \\
053050 & 27.6 & 057000 & 135 \\
\hline
\end{tabular}

Table 17.--Extreme values of water and suspended-8ediment data for the upper Rio Grande de Loiza basin for the 1984,1985 and 1986 water years (mg/L, milligrams per liter,tons/d, tons per day, $\%$,percent)

\begin{tabular}{|c|c|c|c|c|c|c|c|c|}
\hline $\begin{array}{l}\text { Station } \\
\text { Number }\end{array}$ & $\begin{array}{l}\text { Samples } \\
\text { Collec- } \\
\text { ted }\end{array}$ & $\begin{array}{l}\text { Particle } \\
\text { size } \\
\text { analysis }\end{array}$ & $\begin{array}{l}\text { Maximum } \\
\text { Concen- } \\
\text { tration, } \\
\text { in } \mathrm{mg} / \mathrm{L}\end{array}$ & $\begin{array}{l}\text { Minimum } \\
\text { Concen- } \\
\text { tration, } \\
\text { in } \mathrm{mg} / \mathrm{L}\end{array}$ & $\begin{array}{l}\text { Maximum } \\
\text { Load, in } \\
\text { tons/d }\end{array}$ & $\begin{array}{l}\text { Minimum } \\
\text { Load, in } \\
\text { tons/d }\end{array}$ & $\begin{array}{l}\text { Maximum } \\
\text { Percent } \\
\text { Sand, } \\
\text { in \% }\end{array}$ & $\begin{array}{l}\text { Minimum } \\
\text { Percent } \\
\text { Sand, } \\
\text { in } \%\end{array}$ \\
\hline $\begin{array}{c}50050900 \\
50051150 \\
50051180 \\
50051310 \\
50053050 \\
50055000 \\
50055650 \\
50056400 \\
50056900 \\
50057000 \\
\text { Tota1 }\end{array}$ & $\begin{array}{r}309 \\
124 \\
114 \\
276 \\
321 \\
261 \\
90 \\
361 \\
122 \\
138 \\
--- \\
2,116\end{array}$ & $\begin{array}{r}6 \\
12 \\
6 \\
18 \\
23 \\
57 \\
2 \\
16 \\
1 \\
11 \\
--- \\
152\end{array}$ & $\begin{array}{r}10,000 \\
56,100 \\
3,300 \\
5,580 \\
28,600 \\
12,300 \\
2,470 \\
8,970 \\
1,640 \\
3,010\end{array}$ & $\begin{array}{l}0 \\
0 \\
0 \\
1 \\
0 \\
0 \\
1 \\
0 \\
0 \\
0\end{array}$ & $\begin{array}{r}54,200 \\
14,300 \\
4,480 \\
52,100 \\
472,000 \\
817,000 \\
774 \\
99,300 \\
2,480 \\
80,300\end{array}$ & $\begin{array}{l}0.20 \\
.01 \\
0 \\
.06 \\
.06 \\
1.3 \\
0 \\
0 \\
0 \\
0\end{array}$ & $\begin{array}{l}66 \\
45 \\
25 \\
75 \\
80 \\
57 \\
18 \\
73 \\
23 \\
29\end{array}$ & $\begin{array}{r}38 \\
8 \\
7 \\
37 \\
12 \\
12 \\
6 \\
10 \\
23 \\
8\end{array}$ \\
\hline
\end{tabular}

\section{REFERENCES}

Briggs, R.P. and Akers, J.P., 1965, Hydrogeologic Map of Puerto Rico and adjacent islands, U.S. Geological Survey Hydrologic Investigations Atlas HA-197, 1 plate.

Guy, H.P., 1969, Laboratory theory and methods for sediment analysis: U.S. Geological Survey Techniques of Wat Resources Investigations, Book 5, Chap. C1, 58 p.

Guy, H.P. and Norman, V.W., 1970, Field methods for measurements of fluvial sediment: U.S. Geological Survey Techniques of Water Resources Investigations, Book 3, Chap. C2, 59 p.

livari, T.A., 1981, A resurvey of sediment deposits in Loíza Reservoir, Puerto Rico: U.S. Department of Agriculture, Soil Conservation Service, 32 p.

Potterfield, G., 1972, Computation of fluvial-sediment discharge: U.S. Geological Șurvey Techniques of Water Resources Investigations, Book 3, Chap. C3, 66 p.
Quiñones-Marquez, Ferdinand, 1980, Limnology of Lago Loíza, Puerto Rico: U.S. Geological Survey Water Resources Investigations Report 79-97, $128 \mathrm{p}$.

Quiñones-Marquez, Ferdinand, Green, B.K., and Santiago, Luis, 1987, Sedimentation survey of Lago Loíza, Puerto Rico, July 1985: U.S. Geological Survey Water Resources Investigations Report 87-4019, 20 p.

Rantz, S.E. and others, 1982, Measurement and computation of stream-flow: Volume 1. Measurement of stage and discharge: U.S. Geological Survey Water-Supply Paper 2175, 284 p.

U.S. Soil Conservation Service, 1980, Loíza Reservoir sedimentation survey: San Juan, Puerto Rico, (unpublished report). 